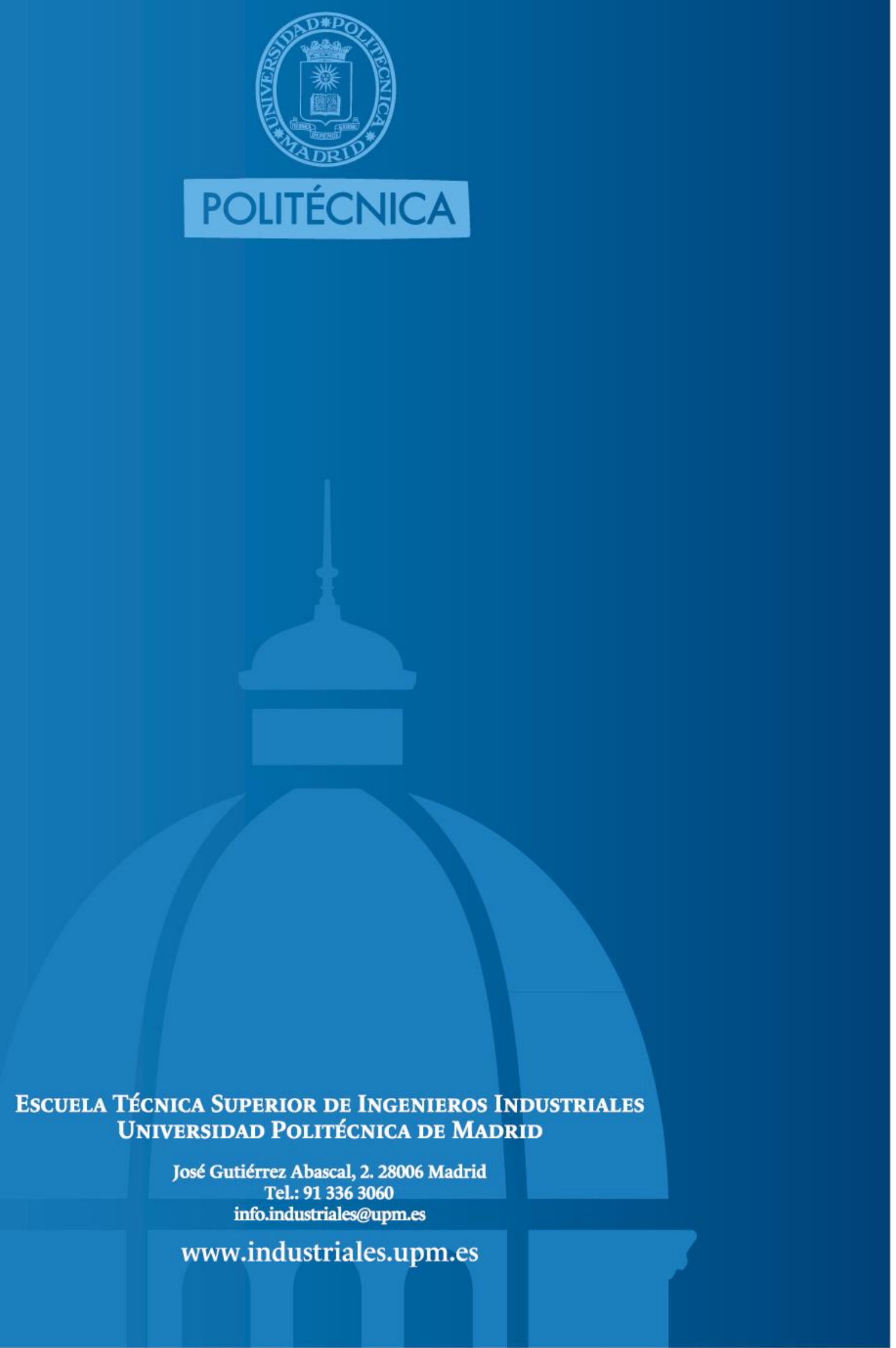

DESIGN, MODELLING, CONTROL AND TELEOPERATION OF HYPERREDUNDANT ROBOTS.

TESIS DOCTORAL PARA LA OBTENCIÓN DEL TÍTULO DE DOCTOR

2020

Andrés Martín Barrio

DIRECTOR DE LA TESIS DOCTORAL:

Antonio Barrientos Cruz

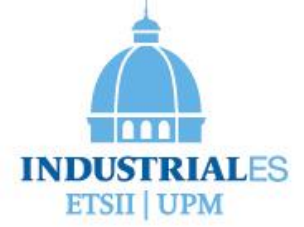





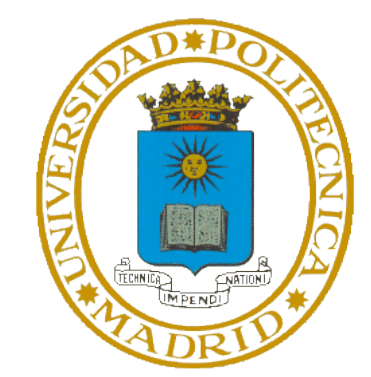

UNIVERSIDAD POLITÉCNICA DE MADRID

ESCUELA TÉCNICA SUPERIOR DE INGENIEROS INDUSTRIALES

CENTRO DE AUTOMÁTICA Y ROBÓTICA

\section{DESIGN, MODELLING, CONTROL AND TELEOPERATION OF HYPER-REDUNDANT ROBOTS DOCTORAL THESIS}

Author:

MsC. Andrés Martín Barrio
Director:

PhD. Antonio Barrientos Cruz 


\section{TRIBUNAL}

Universidad Politécnica de Madrid

Escuela Técnica Superior de Ingenieros Industriales

Title:

Design, Modelling, Control and Teleoperation of Hyper-Redundant Robots

Author:

Andrés Martín Barrio

Director:

Antonio Barrientos Cruz

Tribunal nombrado por el Mgfco. Y Excmo. Sr. Rector de la Universidad Politécnica de Madrid el día .....de ...........de 2020.

Presidente:

Vocales:

Secretario:

Suplentes:

Calificación de la Tésis:

EL PRESIDENTE

LOS VOCALES

EL SECRETARIO 
Available evidence may be insufficient to determine the original causes. 



\section{Acknowledgements}

En estos agradecimientos voy a escribir sin ataduras, porque sin apoyo, no habría lugar para esta tesis, y porque sin emoción tampoco existiría la ciencia.

En primer lugar, me gustaría dar las gracias a Antonio Barrientos, mi Profesor de máster y de mi tesis doctoral por su dedicación y por la confianza que ha depositado en mí. Gracias por sacar mi mejor versión a nivel profesional, por tu contagiosa pasión por la robótica, por enseñarme a no juzgar más que con rigor, por buscarme a la hora de participar en proyectos, por tus precisos y valiosos consejos, y por todo lo que he aprendido de ti a nivel personal y profesional en los últimos 5 años. Me siento orgulloso de tenerte como referente y espero haber sido digno de tu enseñanza.

También, quería mencionar a mi grupo de investigación. Jaime, gracias por tu ayuda, por tus consejos, por valorar mi tesis y por todo lo que he aprendido de ti. Silvia, qué decirte, si lo has vivido todo junto a mí; grazie por tu pazienza, por tu generosità, por las risas y por haber sabido aguantarme en los momentos difíciles. Juanje, gracias por tu disposición a ayudarme siempre que te lo he pedido, por acompañarme en mis divagaciones, por los partidos de tenis y por dejarme entrar en las realidades virtuales; en esas, siempre podrás dominar el mundo. Pablo, de la misma manera, gracias por tu ayuda desinteresada y por tu apoyo, por ponerme en cintura con tu sinceridad y por tu compañía mientras trabajamos. Igualmente, gracias a Mario por tu profesionalidad y por el tiempo que me has dedicado, Christyan por ayudarme desinteresadamente, y también a Jorge por tu compañía en situaciones de auxilio. También quiero acordarme de Carlos, Rosa y Paloma por haberme hecho sentir como en casa. Por otro lado, mi especial mención al resto de profesores, compañeros de doctorado y técnicos de laboratorio que me hayan acompañado en esta etapa dentro de la Universidad Politécnica de Madrid.

Helmut, thank you very much for offering me the possibility to work with you in Bristol. You made me feel like home and gave me the best resources and ideas to finish this thesis. Also, to the researchers of the Bristol Robotics Laboratory and especially to Euan and Hatem.

Esta tesis ha sido resultado de la colaboración conjunta, tanto directa como indirecta, de mis alumnos de Trabajo Fin de Grado y Máster: Álvaro Vara Mateos, Javier Martín García, Manuel Faysse, Montserrat Díaz-Carrasco Díaz, Jesús Pareja Muñoz, David Gutiérrez Vivas, David Ramos Porres, Silvia Jiménez Gómez, Agustín Ramírez Blanco, Laura Bachiller Matallana, Roberto Abia Carrasco, Adrián Barra Durán y Antonio del Abril Mur. Y especialmente a Cecilia Martínez Martín, por 
acordarse siempre de mí y haberme abierto las puertas de una nueva etapa en mi vida. Gracias a todos vosotros.

Con esta tesis, culmino 27 años incansables de estudio. Por lo tanto, quiero acordarme de todos los profesores apasionados tanto en el colegio como en la universidad que han dejado una huella en mí desde pequeño. Y también de todos los compañeros que, de una manera u otra, intentaron minarme hace ya mucho tiempo. Hoy soy mejor gracias a todos vosotros.

A mis amigos: Alber, gracias por haber estado conmigo desde que tengo memoria; Sergio, gracias por estar pendiente de mis avances y preocuparte siempre por mí; Javi, en esta tesis sale Moira si buscas bien, gracias por saber escucharme y comprenderme; Velázquez, muchas gracias por tu sinceridad, te dedico este gusiluz; Paco, gracias por tu apoyo siempre; Yari, gracias por saber escucharme y preocuparte por mí; James, unconditional incorporation, thank you for being so supportive and always sticking up for me; Eric, cuenta con uno de estos robots para la policía; Guillermo y Vicky, gracias por haberme acogido como parte de vuestra familia; y a Alex., Elena, María, Rul, Dani, Sandra, Leo, Miki, por haber formado parte de este periodo de mi vida.

Para finalizar, quiero dedicar este trabajo a mi familia. Mamá, si estoy aquí es gracias a ti. Gracias por dármelo todo, por enseñarme a vivir, y a sobrevivir, por tu cariño incondicional, por ser luz en mis oscuridades y por ser el mejor modelo a seguir. Papá, si algo me diferencia de los demás, es que me apasiona lo que hago, y eso lo he aprendido de ti. Gracias por enseñarme la importancia del trabajo duro desde la pasión, y sin concesiones. Abel, hermano, gracias por estar siempre de mi lado y por acompañarme en mis éxitos. Por último, también a todos los demás que, bien estéis conmigo o bien ya no pueda veros, espero que me miréis con el mismo orgullo que tengo yo de teneros a vosotros. A mis tías y tíos: Matilde, $M^{a}$ Ángeles, $M^{a}$ Eugenia, Carmen, Nadim, José Luís y Javier, por quererme y cuidarme como a un hijo; a mis primas y primos: Santiago, Ángel, Luis, Enita, Pablo y Clara, por formar parte de mis recuerdos más felices; a los pequeños: Ángela, Julia, June, Mateo y Alonso, por representar lo mejor de lo que está por llegar; y a mis abuelos: Santiago, $M^{a}$ Ángeles, Teo y Pepe, por ser inmortales en mi memoria y por plantar la semilla que hoy me ha permitido confeccionarme en la persona que siempre he querido ser.

A todos vosotros os dedico esta tesis doctoral. 


\section{Abstract}

This work studies highly-articulated robots, specifically those manipulators that are hyperredundant, continuous, and soft. These devices are well-known for being fault-tolerant or having better kinematic skills to avoid obstacles and move in unstructured spaces, compared to traditional ones. However, they face multiple engineering challenges that are acknowledged in this document; from their design, modelling process and control, to their teleoperation. Therefore, this work firstly proposes a new mathematical definition for these robots and a novel classification. An updated stateof-the-art also grants a complete overview based on a rigorous scientific method. Different design ideas are provided to construct these robots, in which finding a compromise between different requirements is essential. In addition, multiple strategies are applied to predict their movements, specifically for continuous and soft robots. These include the real-time Finite Element Method which stands out to be the most promising, flexible, accurate, efficient and complete tool. Moreover, two novel control algorithms are originally introduced: the Natural-CCD and SCD methods. They are based on very simple principles to efficiently and flexibly customize the control of highly-articulated mechanisms including discrete, continuous, soft, growing, cooperative and even branching structures. These algorithms are designed to solve different control strategies like the inverse kinematics, following of trajectories, shape and follow-the-leader controls or motion planning with obstacle avoidance. Interestingly, a set of primal behaviours were also found based on the kinematics of these robots, constituting a new perspective to control their movements. Finally, disruptive immersive technologies and natural language tools were found to be the best suited to teleoperate these complex manipulators in terms of situational awareness and objective efficiency. In summary, highly-articulated robots are quite challenging but they are expected to play a very important role in the future to come. 


\section{Resumen}

Este trabajo estudia a robots muy articulados, específicamente aquellos que son hiper-redundantes, continuos y blandos. Estos dispositivos son conocidos por ser tolerantes a fallos o presentar mejores habilidades cinemáticas que los robots tradicionales para evitar obstáculos o para moverse en entornos desestructurados. Sin embargo, también presentan múltiples retos a nivel de ingeniería que se analizarán en este documento; desde su diseño, modelado y control, hasta su teleoperación. Por lo tanto, este trabajo primero propone una nueva definición matemática para estos robots, así como una clasificación actualizada. También, se proporciona un estado del arte actualizado para dar una visión integradora basada en un riguroso método científico. Seguidamente, diferentes ideas de diseño han sido propuestas para construir estos robots, en los que habitualmente es esencial encontrar un compromiso entre diferentes requerimientos. Asimismo, se han aplicado múltiples estrategias para predecir sus movimientos, específicamente para aquellos robots continuos y blandos. Por ejemplo, se ha usado el Método de Elementos Finitos en tiempo real, que destaca por ser la herramienta más prometedora, flexible, precisa, eficiente y completa. También, dos nuevos algoritmos de control han sido originalmente propuestos: el Natural-CCD y SCD. Están basados en principios muy básicos capaces de controlar y personalizar de una forma eficiente y flexible el control de estos mecanismos, entre los que se incluyen aquellos discretos, continuos, blandos, extensibles, cooperativos e incluso arborescentes. Estos algoritmos han sido diseñados para dar solución a diferentes estrategias de control como la cinemática inversa, el seguimiento de trayectorias, controles basados en forma o en el seguimiento de un líder, e incluso para planificación de movimientos y evasión de obstáculos. Curiosamente, también se encontraron una serie de comportamientos primitivos para este tipo de robots que han dado lugar a una nueva perspectiva para controlar sus movimientos. Finalmente, se ha descubierto que las disruptivas tecnologías inmersivas y el lenguaje natural son las mejores herramientas para teleoperar estos manipuladores en términos de conciencia de la situación y de una eficiencia objetiva. En resumen, los robots altamente articulados suponen un gran reto, pero a su vez resultan prometedores y se espera que jueguen un papel fundamental en el futuro que está por llegar. 


\section{Contents}

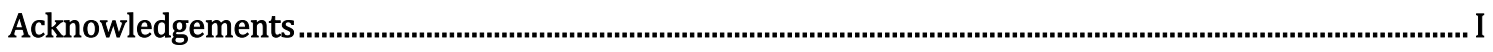

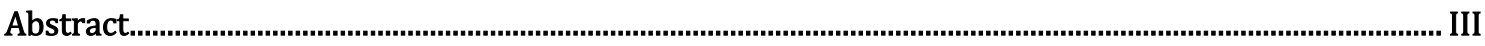

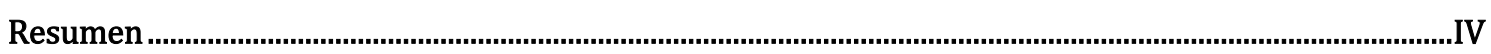

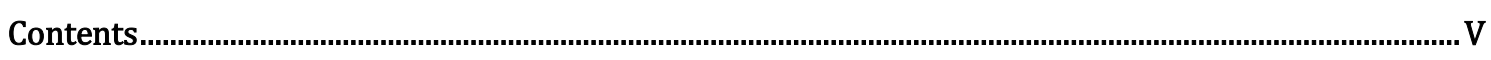

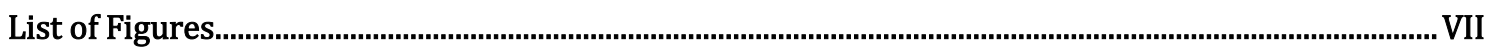

List of Tables ............................................................................................................................

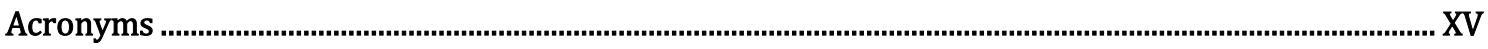

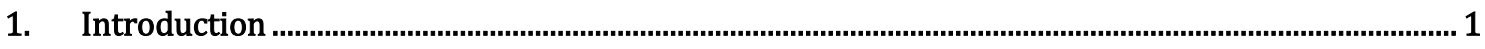

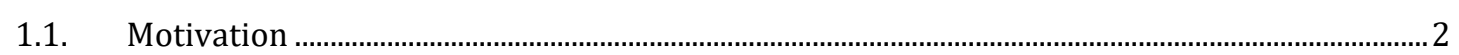

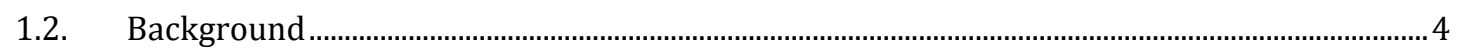

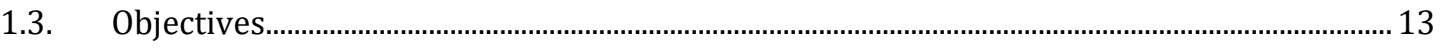

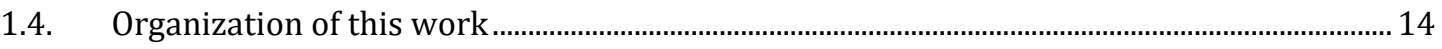

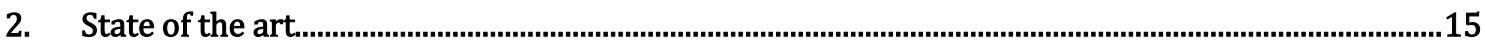

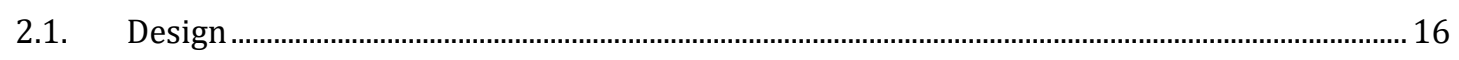

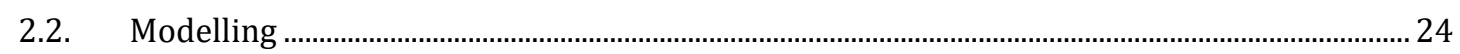

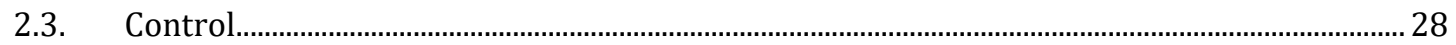

2.4. Teleoperation .................................................................................................................... 31

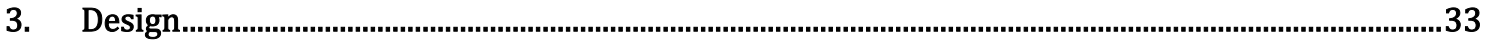

3.1. Mach, a discrete hyper-redundant robot............................................................................... 34

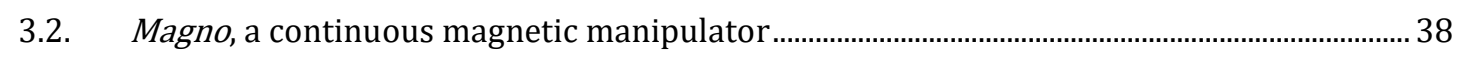

3.3. Kуma, a tendon-driven soft robot ....................................................................................... 40

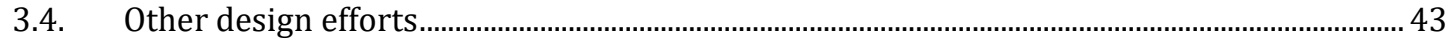

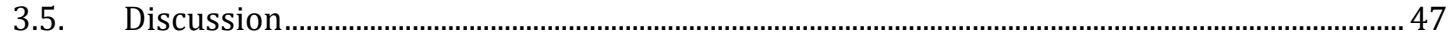




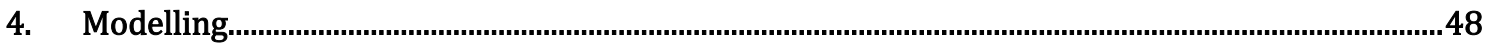

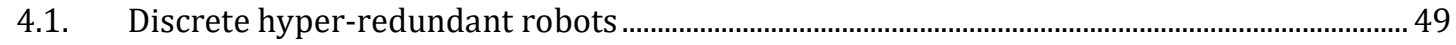

4.2. Continuous robots........................................................................................................................ 51

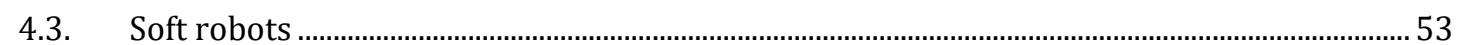

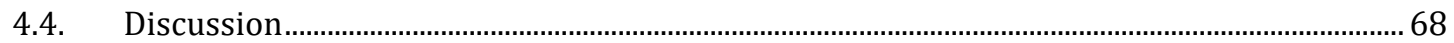

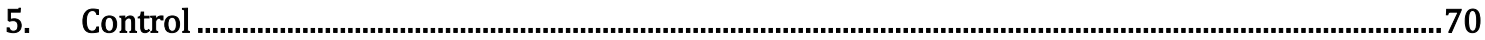

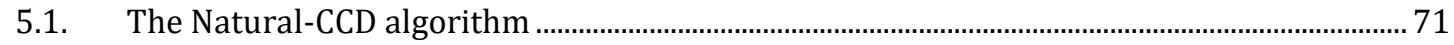

5.2. Extension to emerging behaviours................................................................................................ 87

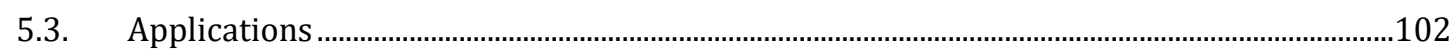

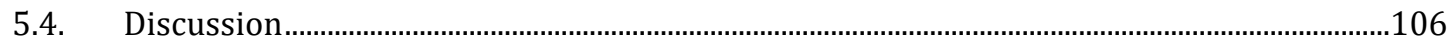

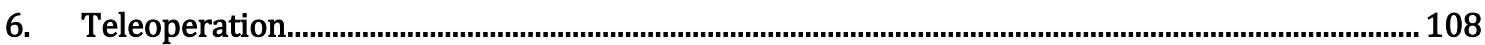

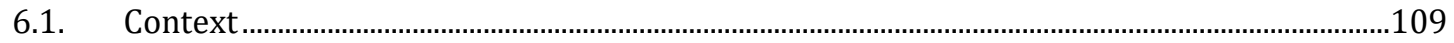

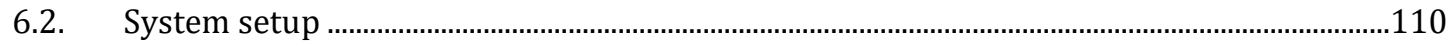

6.3. Human-robot interfaces .................................................................................................................112

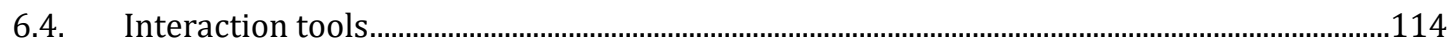

6.5. Results and validation ...................................................................................................................119

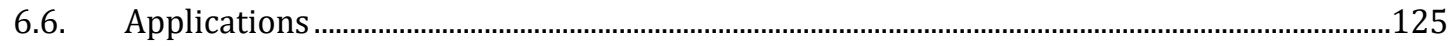

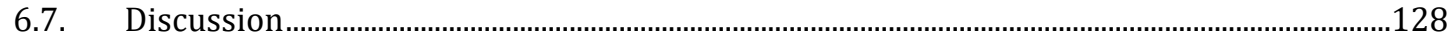

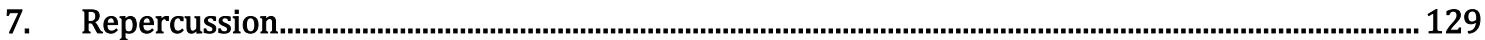

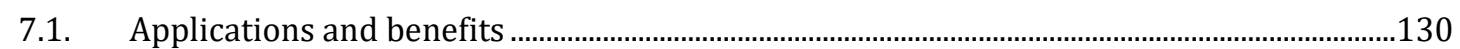

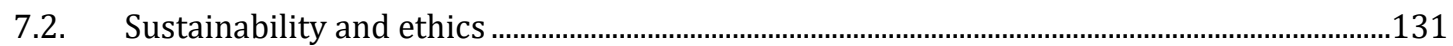

7.3. Achievements..........................................................................................................................132

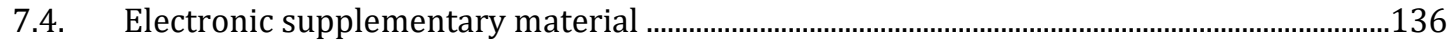

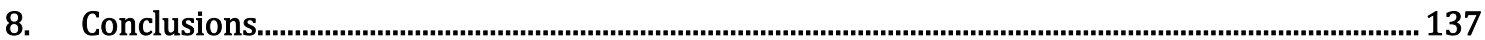

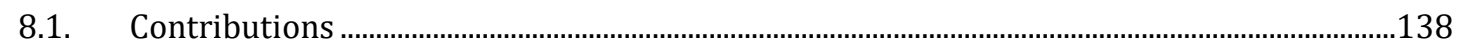

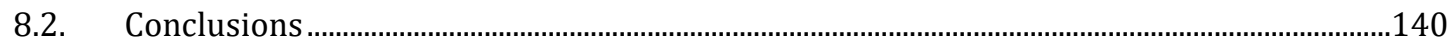

8.3. Future research lines ......................................................................................................................143

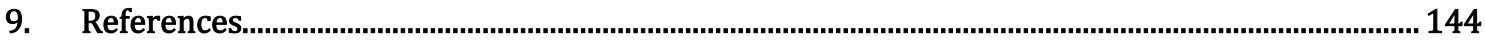




\section{List of Figures}

Figure 1.1. Concept design of a magnetic hyper-redundant robot [1]

.2

Figure 1.2. Robot classification according to the number of DoF, number of modules, their materials and actuation. Several robotic systems have been used to illustrate this classification [6-11]. The areas in white or grey are empty sets.

....4

Figure 1.3. Front view of different types of robots with rotational joints in a three-dimensional space $(\mathrm{m}=3$ ). (a) Non-redundant robot type $\mathrm{n}<\mathrm{m}$. (b) Non-redundant robot type $\mathrm{n}=\mathrm{m}$. (c) Redundant robot Type $n>m$. (d) Hyper-redundant robot type $n=2 m$.

Figure 1.4. Research impact of the types of hyper-redundant robots in recent years. Source: Web of Science..

Figure 2.1. ACM III, one of the first hyper-redundant robots made in 1972 by Hirose [103] ............... 16

Figure 2.2. Relevant mobile hyper-redundant robots. (a) MAKROPlus [106], (b) Genbu [107], (c)

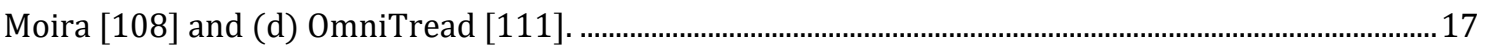

Figure 2.3. Relevant mobile hyper-redundant robots. (a) Amphibot II [112], (b) Anna Konda [113],

(c) Slim Slime [114] , (d) Kulko [115], (e) and (f) ACM R5 [22] .............................................................17

Figure 2.4. Woodstock, a very compact and strong robot [40] ............................................................. 18

Figure 2.5. Series II X125, one of the few and most advanced industrial hyper-redundant robots... 18

Figure 2.6. Elephant's trunk by Hannan and Walker [21] ................................................................... 19

Figure 2.7. Tendril, made by NASA to perform minimally invasive tasks in space [41] ........................19

Figure 2.8. ET ARM, actuated by artificial muscles [138] .....................................................................2

Figure 2.9. Robot with 25 DoF for inspection and reparation tasks in wind turbines [98] .................. 20

Figure 2.10. Air-Octor, a continuous manipulator robot with multiple sections [140]. ........................ 21

Figure 2.11. Octarm V, a soft robot inspired in an octopus tentacle [141] ............................................ 22

Figure 2.12. Bionic Handling Assistant (BHA), pneumatic soft robot with high dexterity made by

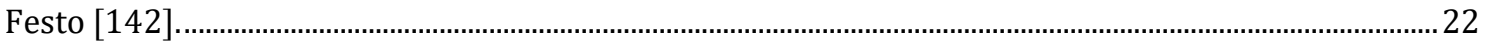

Figure 2.13. Soft robot inspired in a tentacle [102] ............................................................................. 23

Figure 2.14. Continuous and soft hyper-redundant robot composed by a braided cover and actuated

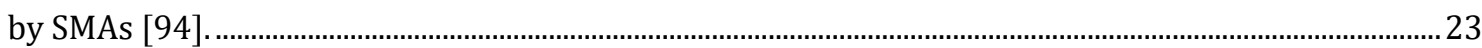

Figure 2.15. Scheme of the main modelling strategies for continuous and soft robots. ..........................24

Figure 3.1. Complete mechanical design of a tendon-driven, discrete and hyper-redundant manipulator with 14 degrees-of-freedom and 7 sections. .........................................................................

Figure 3.2. Free body diagram of the required torque exerted by the first joint to balance a payload and the weight of the robot body. ...................................................................................................................... 
Figure 3.3. Perspective (a) front and (b) back view of the complete design of the hyper-redundant

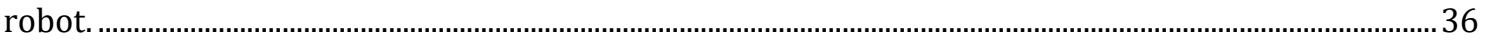

Figure 3.4. Customized design of different electronic boards. (a) Motor drivers for a closed-loop control using encoders. (b) Electronic board to supervise the limit switches. (c) Central controller to manage all the communications. (d) $24 \mathrm{~V}$ power board to supply the motors. (e) $9 \mathrm{~V}$ power board to supply energy to the rest of the electronic boards. ....................................................................................3 Figure 3.5. The magnetic modules (2) are alternated facing opposite poles throughout the flexible core (1), allowing prismatic movements like a spring would do...............................................................3 Figure 3.6. Full mechanical model of Magno, a continuous manipulator with bending and prismatic capabilities. (a) By actuating each cable independently or by pairs, it is possible to control bending. (b) If the three cables of each section are actuated simultaneously, then it is possible to control prismatic movements.

Figure 3.7. (a) Mechanical model of the soft manipulator Kyma, composed by 4 equally spaced modules actuated by tendons. (b) Detail section of one module. Dimensions in centimetres. ........... 40 Figure 3.8. Soft PVC bellow used to construct the main structure. The morphology allows both bending and prismatic capabilities.

Figure 3.9. Different configurations to distribute the cables in a single module. Same colour dots represent the movement of a motor and a cable in two directions, black axis represent independent actuation rotations and orange ones the combination of all of them. 41

Figure 3.10. Inter-modular disk made from fiber-reinforced plastic...........................................................4 41

Figure 3.11. Actuation box with 12 stepper motors located in two parallel planes.............................. 42

Figure 3.12. Three-dimensional magnetic field created by the conjunction of multiple magnetic variable sources to control the movements of a magnetorheological, susceptible robotic device..... 43 Figure 3.13. Composition of a squared signal from the sum of multiple sinusoidal signals obtained from the Fourier transform .......................................................................................................................... 44 Figure 3.14. Epicycle resulted from of the rotation of two segments, represented in red. .................... 45 Figure 3.15. A set of multiple segments that rotate can result in a complex trajectory under the initial

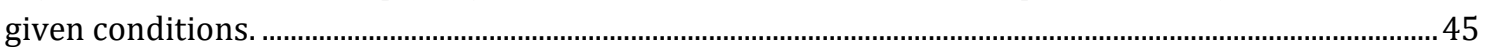
Figure 3.16. The trajectory of the tip of a double pendulum is a chaotic system...................................46 Figure 4.1. Denavit-Hartenberg convention to model rigid and discrete manipulators.........................50 Figure 4.2. Sensor BNO055 and multiplexor TCA9548A. ........................................................................51 Figure 4.3. Experimental comparison of the real pose of the robot obtained with an Optitrack $\AA$ system and the estimated pose of the robot with the presented method..................................................52 Figure 4.4. Main different stages to model the soft robot Kyma using the real-time Finite Element Method.

Figure 4.5. Main components to model in the SOFA environment. (a) Deformation model. (b) Actuation model. (c) Collision model. (d) Visual model. ........................................................................56 Figure 4.6. (a) Full-order deformation mesh with 120000 tetrahedra, infeasible for real-time applications. (b) The heuristic method adapts a model with 1650 elements to match the real robot behaviour. (c) The Model Order Reduction decreases the number of state variables of the model to 1886 while retaining its original characteristics. 57 Figure 4.7. Actuation system based on three tendons per section. (a) Not actuated, (b) side view of the left-tendon displacement and (c) multiple-tendon displacement. ......................................................59 Figure 4.8. Transmission system based on (a) tendons without guidance and (b) the same system

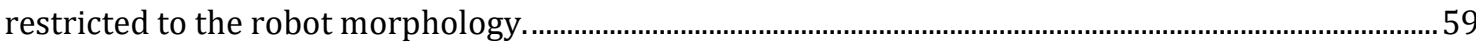
Figure 4.9. Side and top view of the complete actuation system of Kyma. Tendons corresponding to the same robot section are represented with same colours........................................................................6 60 Figure 4.10. Difference between (a) the real robot behaviour with physical restrictions and (b) the undesired model without self-collision restrictions. 61 
Figure 4.11. Self-collision restriction model for one section. (a) Robot in rest pose. (b) Actuated robot. Different colours are used to depict different collision systems. This model describes better the real robot behaviour.

Figure 4.12. Front and perspective view of the Kyma model interacting with its own supporting structure in a zero-gravity environment.

Figure 4.13. Soft robot compliance with a fixed obstacle in different situations. ...................................... 63

Figure 4.14. Kyma interacting with a flexible toroid-shape object.............................................................. 64

Figure 4.15. Lift of a non-fixed object from the ground using the robot body. .........................................64

Figure 4.16. Three of a total of twelve infrared-sensitive passive markers, used by the motion capture

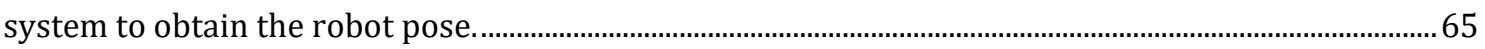

Figure 4.17. Comparison of a single test of the real robot (left) versus the obtained model (right). 66 Figure 4.18. Real robot workspace representation for 100 iterations and validation of the FEM model. The absolute error is compared to the Euclidean maximum distance of the robot from a non-actuated pose.

Figure 5.1 The Cyclic Coordinate Descent algorithm rotates every control point so the distance between the end-effector and the desired target is minimized [11].

. .71

Figure 5.2. Movement of a hyper-redundant robot with 10 rotational joints (a) from a straight configuration to a desired position, (b) using the Natural-CCD algorithm (c) and the Natural-SCD algorithm . .73

Figure 5.3. Kinematic growing process from a discrete perspective. (a) When a module extends its length $L i$ close to a predefined maximum constraint, (b) a new control point is added to the configuration, increasing the robot maneuverability.

Figure 5.4. Movement of a planar robot with 10 rotational joints using the Natural-CCD algorithm. (a) Initial straight configuration. (b) Intermediate configurations. (c) Final configuration and the endeffector path representation........................................................................................................................ 74

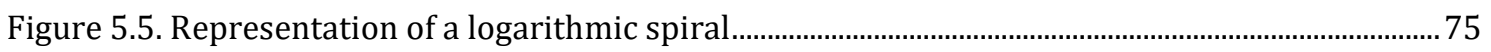

Figure 5.6. Curvature-arc length test results for the movement of a robot with 10 links using the Natural-CCD algorithm from a straight configuration and with a desired end-effector position closed

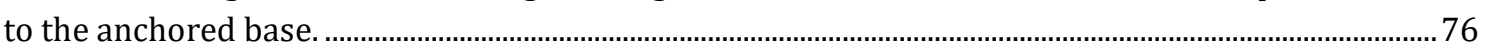
Figure 5.7. The Fibonacci spiral constructed from squares with same size as the Fibonacci sequence.

Figure 5.8. Comparison of the Fibonacci spiral constant angle $\beta_{\mathrm{f}}$ and the obtained angle $\beta$ from the resulting path presented in Figure 5.4 versus the number of links of the robot....................................... 78 Figure 5.9. Comparison of (a) the Fibonacci spiral and (b) the resulting end-effector path using the Natural-CCD algorithm . .78

Figure 5.10. The Fibonacci spiral path and representation of the circumferences around the robot configurations. 79

Figure 5.11. Interface created in MATLAB to customize and supervise the control of the hyperredundant robot.

Figure 5.12. Movement of a robot with three rotational joints using the Natural-CCD from a straight configuration to a given desired point, where $\mathrm{q}_{1}, \mathrm{q}_{2}, \ldots, \mathrm{q}_{\mathrm{n}}$ are the robot joints from the base to the end-effector. (a) Representation of the intermediate configurations. (b) Convergence of the endeffector to the desired position measured as a Euclidean distance. (c) Angle variation for each joint.

. .80

Figure 5.13. Movement of a hyper-redundant robot with 10 rotational joints and 3 DOFs per joint using the Natural-CCD. (a) Initial configuration and desired position and orientation for the endeffector. (b) Final configuration in a 3D perspective. (c) Top view of the final configuration. . .80 
Figure 5.14. Movement of a robot with 5 rotational joints and 2 rotational and prismatic joints (yellow) from a straight configuration to a desired position using the Natural-CCD. (a) Initial straight configuration. (b) Intermediate configurations. (c) Final configuration....................................................8 80 Figure 5.15. Simulation of failure of 4 joints (red) over a total of 12 rotational joints to study fault tolerance for hyper-redundant robots- (a) Initial straight configuration. (b) Intermediate configurations. (c) Final configuration. 81 Figure 5.16. Trajectory planning of a robot with 20 rotational joints and 3 DOFs per joint (60 DOFs) using the SCD. (a) Desired straight-line trajectory. (b) Final configuration and end-effector path for the line trajectory. (c) Desired helical trajectory. (d) Final configuration and end-effector path for the helical trajectory. 81 Figure 5.17. Combined use of Natural-CCD and potential fields to avoid the obstacles modelled as consecutive spheres. (a) Initial robot configuration, obstacles and desired position. (b) Final configuration and path. 82 Figure 5.18. Motion planning for hyper-redundant robots in complex environments. No collisions occurred during the execution. (a) Initial robot configuration in an environment with two complex obstacles and representation of the desired position and a valid collision-free trajectory for the end effector. (b) Final robot configuration and path for the previous scenario. (c) Initial configuration, desired position, and collision-free trajectory for the end effector in other scenarios with walls and

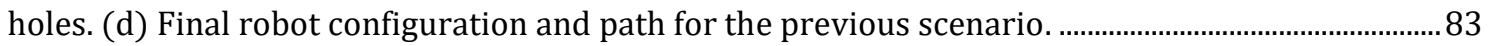
Figure 5.19. Use of the natural-CCD algorithm for resolution of a complex task with a lot of constraints. No collisions occurred during the execution. (a) Initial configuration of a robot with 9 rotational joints and 3 DOFs per joint. A mechanical failure is simulated in 2 joints (second and third from the base) and the last joint is also prismatic. The workspace includes 9 consecutive obstacles that have to be avoided. Then, the robot must conduct a linear trajectory with a given orientation. (b) Final robot configuration in a 3D perspective. (c) Final robot configuration in a front view..............84 Figure 5.20. Mean computational time of the inverse kinematics problem resolution versus the number of joints for 1, 2, or 3 DOFs per joint (a) for given random positions (b) and for given random positions and orientations.

Figure 5.21. Computational time comparison of the state-of-the-art methods and the natural-CCD

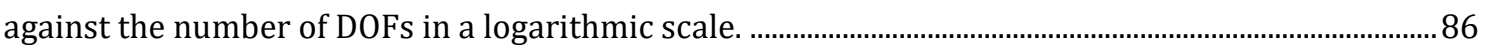
Figure 5.22. Relevant points in a kinematic chain and the location of an external reference Q ..........87 Figure 5.23. (a) The inverse kinematics problem requires a single reference to optimize the endeffector location. (b) Shape control requires a multi-variable reference (grey) to define the desired pose of the robot. 89

Figure 5.24. Different strategies for to perform a shape control. The current state of the robot (green) and the reference (grey) are the same in both cases. (a) The decoupled strategy (c) leads to a fast converging solution. And (b) the coupled strategy (d) leads to an oscillating-wave movement........90 Figure 5.25. Oscillating-wave movement of robots with the same length and different degrees-offreedom (DoF) moving at the same velocity. Also, convergence between the end-effector and the desired point $\|Q-P(q n)\|$. (a) 5 control points lead to 1 period, (b) 10 control points lead to 2 periods and (c) 20 control points lead to 4 periods. 91 Figure 5.26. (a) Initial configuration of the robot, an static obstacle and the desired end-effector location. (b) The robot configuration is locally repelled to avoid the obstacle, but still reaches the reference with its end-effector. 92 Figure 5.27. (a) Obstacle approaching to a static configuration of the robot. (b) The robot configuration is automatically adapted to locally repel the dynamic object. (c) A growing robot can add new control points (green) to easily avoid the same obstacle while the pose remains static and more energy-efficient. 92 
Figure 5.28. (a) Initial configuration of the robot. (b) Intermediate configurations of the surrounding behaviour. (c) Final configuration with self-collision constraints. ............................................................ 93 Figure 5.29. (a) Object surrounding performed by a regular hyper-redundant robot. (b) A growing robot can reach farther objects and adjust its shape to tight around irregular morphologies............94 Figure 5.30. (a) A growing robot could customize their structure using branches depending on the application. (b) This structure allows the robot to grab complex objects by using the surrounding behaviour on each arm independently. Shadows are depicted in grey to ease the spatial understanding. 94 Figure 5.31. (a) Initial configuration of a hyper-redundant robot. (b) The straightening behaviour

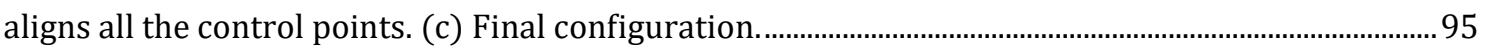
Figure 5.32. (a) Initial configuration of a hyper-redundant robot. (b) The folding behaviour retracts the configuration. (c) Intermediate configuration. (d) Final configuration.............................................96 Figure 5.33. (a) Initial configuration. (b) The end-effector follows a straight line, perpendicular to the robot configuration using the end-effector attraction behaviour. (c) And the resulting movement corresponds to a helical circumnutation. Shadows are depicted in grey to ease the spatial understanding. 97

Figure 5.34. Hyper-redundant robot guided using the follow-the-leader control through a set of pipes for inspection by the section-based approach $(A 2)$. If there is a gas leak, the robot can use the eventbased approach $(A 3)$ to locally repel it. 98 Figure 5.35. (a) Initial robot configuration and follow-the-leader trajectory. (b) The section-based approach ( $A 2$ ) divides the robot in two sections with different attraction behaviours ( $B 19$ and $B 18$ ). (c) If an external perturbation takes place, (d) the event-based approach ( $A 3)$ will readjust the shape of the robot using the local repulsion behaviour (B18). .. 99 Figure 5.36. A growing robot taking a distant sample from a spacecraft. The robot can surround the object with the whole-body and bring it closer using the combined approach ( $A 4)$. Then, the configuration can be changed using the time-based motion strategy $(A 1)$ in order to release it.... 100 Figure 5.37. (a) A regular hyper-redundant robot cannot reach very distant objects. (b) But growing robots can extend their length for that purpose. (c) The combined motion strategy ( $A 4)$ can be used to fuse the surrounding and folding behaviours ( $B 11$ and $B 17)$. It can be useful to grab and bring an object closer. (d) Then, the time-based approach ( $A 1$ ) let the configuration change using the straightening behaviour (B11) to release the object when desired. 101 Figure 5.38. (a) Step response for the motor identification can fit to a first or a second-order system. (b) Control scheme for the motors. Two feedback controllers are used both for velocity and position and a feed-forward scheme is implemented to correct undesirable delays in the response. ............ 103 Figure 5.39. (a) The rotations are defined by two angles $\alpha$ and $\beta$ which do not directly represent the rotation axes, but the direction and the magnitude of the rotation, respectively. (b) Transformation of the pull point P to P' after a joint rotation. (c.1) Difference between the real displacements of the cables of the same module versus the angle magnitude. (c.2) Error between the real displacements and the linear assumption 104

Figure 5.40. Shape control applied with a teleoperation scheme based on Mixed Reality................. 105

Figure 6.1. Bilateral scheme for the robot teleoperation...................................................................... 110

Figure 6.2. Kyma, a soft continuous manipulator robot actuated by tendons...................................... 111

Figure 6.3. Conventional interface. Here the scene is displayed in two dimensions by a monitor. The robot is commanded to move using a keyboard and its pose is displayed in the interface in real-time. 113

Figure 6.4. Immersive interface. The setup is nearly the same than in the conventional interface but using a VR headset instead of a monitor. 113 Figure 6.5. Controller strategy. In this interaction tool, a gaming controller is used to select and move the robot joints one by one using buttons and axis. 115 
Figure 6.6. Master-slave strategy. Here, a little replica of the virtual robot (master) is manipulated so the bigger one (slave) is identically moved towards the desired reference.......................................... 116 Figure 6.7. Local gestures strategy. The user's hands are tracked using a Leap Motion system so they can be used to directly interact with the virtual robot 116 Figure 6.8. Remote gestures strategy. In this setup, the user can select the joint to move by pointing with a virtual laser that emanates from the left index finger. Then, the selected joint remotely moves when the right hand is opened. 117

Figure 6.9. Voice commands strategy. The VR headset records the user's voice to recognize simple commands in order to select the joint to move, its speed and the direction. 118 Figure 6.10. Gestures and voice strategy. This strategy uses the voice to select a joint and hand gestures to remotely move it. 118 Figure 6.11. Results for the human-robot interfaces test. (a) Efficiency in terms of precision and time, (b) situational awareness, (c) subjective visual feedback and (d) subjective preference. 120 Figure 6.12. Results for the interaction-tools test. (a) Efficiency in terms of precision and speed, (b) workload, (c) subjective preference, (d) subjective importance of variables in workload, (e) subjective and objective performance comparison and (f) previous experience influence in performance. 122

Figure 6.13. Previous experience influence in efficiency both for the human-robot interfaces and the interaction tools experiments. 124

Figure 6.14. (a) Boiler room with the robot prepared to perform an inspection. (b) First-person view of the robot and the thermal camera located in the end-effector. (c) Two sensors were used in the real environment: an Optrix PI thermal camera and a Logitech RGB camera. (d) On the other hand, the real scenario was reconstructed using a Kinect and the software RTAB-Map. (e) Then, it was imported in Virtual Reality with a model of the robot. (f) The operator uses the HTC Vive VR headset and the Leap Motion controller to teleoperate the real robot from a safe location. 125 Figure 6.15. (a) The operator performs a shape control using his bare hands to interact with the virtual robot, a replica of the real one with the same kinematics and physical restrictions. (b) Using Mixed Reality, the real robot (slave) is moved adopting the same pose as the virtual one (master). The thermal camera located in the end-effector let the operator supervise the temperatures of the boiler room from a safe and remote location. 126 Figure 6.16. The Hololens device, a head mounted display intended for Augmented and Mixed Reality experiences 127

Figure 6.17. Hologram overlapped to the real robot in order to command a shape-control using the Hololens device. Both gestures and voice commands were used to move the hologram.

Figure 7.1. Main principles of the sustainable development.

Figure 7.2. Concentric tube continuum robot. Source: Continuum Robots Laboratory, University of Toronto Mississauga. 


\section{List of Tables}

Table 1.1: Properties and capacities of the different types of hyper-redundant robots.

Table 2.1. Most representative models of hyper-redundant manipulators in the state of the art......16 Table 4.1. Main node hierarchy and a bullet list of the principal components to model Kyma in the SOFA environment alongside their descriptions. .55

Table 4.2. Main parameters of Kyma to construct the FEM model and a heuristic adjustment to decrease the computational cost.

Table 4.3. Analysis of the model thickness versus the number of FEM elements...................................58 Table 4.4. Validation results of the FEM Model for 100 random pose iterations. Actuated control points are numbered from the base to the tip as 1 to 4 respectively. $h$ : distance of the control points to the base. Dmax: maximum separation of the control points from the zero-angle pose. $x 1$ : mean error using the heuristic method. $x 2$ : mean error using the Model Order Reduction method. xmin: minimum error. $x \max$ : maximum error. Errors are accumulated from control points 1 to 4 . Units in millimetres.

Table 5.1. The Natural-CCD algorithm. $\mathrm{p}_{\mathrm{e}}$, current end-effector position; $\mathrm{p}_{\mathrm{f}}$, desired end-effector position; $\mathrm{q}_{\mathrm{i}}$, current joint; $\mathrm{N}$, number of joints; $\theta$ rotation angle; $d$, direction vector; $\mathrm{k}$, variable to reduce the value of the rotation angle; $\mathrm{p}_{c}$, position of the current joint; $\theta c$, angle between the current link to be moved and the previous one; $\theta \max$, maximum angle upper bound. 72 Table 5.2. The SCD algorithm. pe, current end-effector position; pf, desired end-effector position; qi, current joint; $\mathrm{N}$, number of joints; $\theta$ rotation angle; $d$, direction vector; $\mathrm{k}$, variable to reduce the value of the rotation angle; pc, position of the current joint; $\theta c$, angle between the current link to be moved and the previous one; $\theta \max$, maximum angle upper bound 73 Table 5.3. Emerging behaviours obtained from different combinations of $[p c, p e, p f]$ in relevant points of the kinematic chain. Complementary behaviours swap the values of $p e$ and $p f$. Some of the most relevant are selected and depicted with the same colours used in later figures. .88 Table 6.1. Mean and variance values of the participant ages and their subjective previous experience (the higher, the more experience).

Table 6.2. Pair-wise p-values to show the significance between the results of efficiency in the interaction tool test. Relations in red do not reject the null hypothesis. CR: controller, MS: masterslave, LG: local gestures, RG: remote gestures, VC: voice commands, GV: gestures and voice. ......... 122 Table 6.3. Pair-wise p-values to show the significance between the results of the workload in the interaction tools test. Relations in red do not reject the null hypothesis. CR: controller, MS: masterslave, LG: local gestures, RG: remote gestures, VC: voice commands, GV: gestures and voice. 
Table 7.2. Publications directly derived from the research activities of this thesis. ............................ 132

Table 7.3. Other relevant publications with limited relation to this thesis. .............................................. 133

Table 7.4. Conference presented in the context of this PhD thesis........................................................ 133

Table 7.5. Other relevant conferences with limited relation to this thesis............................................. 133

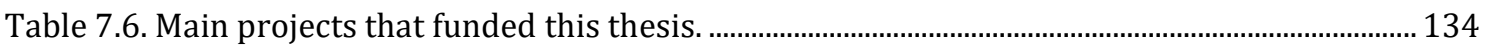

Table 7.7. Scholarship granted to obtain the International Mention for this PhD thesis.................... 134

Table 7.8. Participation as assistant professor in educational courses.................................................. 134

Table 7.9. Participation as reviewer in scientific journals and conferences. .......................................... 134

Table 7.10. Co-supervised BSc and MSc projects during the execution of this thesis........................... 135

Table 7.11. Electronic supplementary material .................................................................................... 136

Table 8.1. Contributions derived from basic principles .......................................................................... 138

Table 8.2. Contributions derived from the design and construction of highly-articulated mechanisms.

Table 8.3. Contributions derived from modelling techniques

Table 8.4. Contributions derived from the kinematic control. ................................................................ 138

Table 8.5. Contributions related to human-machine interfaces ............................................................ 139

Table 8.6. Contributions derived from the teleoperation of highly-articulated manipulators.........139

Table 8.7. Contributions derived from the exploration in new paradigms. ............................................139 


\section{Acronyms}

2D/3D Two/Three Dimensional

ACiD Adaptive Coordinate Descent

ANN Artificial Neural Network

ANOVA Analysis of Variance

AR Augmented Reality

CAD Computer Aided Design

CAN Controller Area Network

CAR Centre for Automation and Robotics

CCD Cyclic Coordinate Descent

CR Controller

CSIC Centro Superior de Investigaciones Científicas

DoF Degrees of Freedom

FBG Fiber Bragg Grating

FEM Finite Element Method

FNN Feedforward Neural Network

FoV Field of View

GV Gestures and Voice

HIC Head Injury Criterion

HMD Head-Mounted Display

IK Inverse Kinematics

IMU Inertial Measurement Unit

LED Light Emitting Diode

LG Local Gestures

MOR Model Order Reduction

MPC Model Predictive Control

MR Mixed Reality

MS Master-Slave

NOTES Natural Orifice Transluminal Endoscopy Surgery

ODE Ordinary Differential Equations

PCC Piecewise Constant Curvature

POLA Principle of Least Astonishment 
PRM Probabilistic RoadMaps

PVC Polyvinyl Chloride

RG Remote Gestures

RGB Red Green Blue

RRT Rapidly-exploring Random Trees

SCD Selective Coordinate Descent

SMA Shape Memory Alloy

SOFA Simulation Open Framework Architecture

TCP/IP Transmission Control/ Internet Protocol

UAV Unmanned Aerial Vehicle

UHMwPE Ultra-High Molecular Weight Polyethylene

UPM Universidad Politécnica de Madrid

VC Voice Commands

VR Virtual Reality 
This chapter presents information to provide a better understanding of the rest of this work. From the reasons that motivate it, followed by some important background concepts to understand its context, the pursued objectives and a brief organization of the rest of the chapters of this thesis.

Further information of this section can be found in an original scientific publication ${ }^{1}$.

${ }^{1}$ Andrés Martín-Barrio et al., Hyper-Redundant Robots: Classification, State-of-the-Art and Issues. Revista Iberoamericana de Automática e Informática industrial, 2018.15(4): p. 351-362. 


\subsection{Motivation}

Early robots were initially designed for manufacturing purposes in industry. They were devised to perform repetitive tasks in highly controlled environments [2]. Traditional serial manipulators were expected to position and orient their end-effector in the three-dimensional (3D) space for different applications. In order to do so, a robot needs to have a minimum number of 6 independent movements, also called Degree of Freedom (DoF) [3]. In robotics, the concept of degrees of freedom is very important. It is defined as the number of independent movements or parameters that define the configuration of a robot [3]. The configuration, also called pose, can be understood as the arrangement of the whole-body of the robot. It is necessary at least $6 \mathrm{DoF}-3$ to determine the position and 3 to determine the orientation - to control the robot's tip in the three-dimensional space.

Simultaneously, other types of manipulators with more than 6 degrees-offreedom were devised. Such manipulators exhibited dexterous kinematic skills, fault-tolerance and higher capabilities to move in unstructured environments. Because of their large number of degrees-of-freedom, they were called hyperredundant robots.

Among their kinematic advantages, this kind of robots can maximize the actuator's transmission of force - finding the solution that best convey the force to the end-effector; the availability of the joint's range - keeping them up as close to their central positions-; the dexterity - proposing solutions that provide greater manoeuvrability to the end-effector-; or the energy saving - managing efficiently speeds and inertias-. On the other hand, due to the high number of DoF, hyper-redundant robots are provided with great versatility to work in complex scenarios. Their morphologies offer a wide variety of solutions to solve movement planning problems in environments with obstacles [4]. Also, these robots are fault-tolerant [5], so to say, if any of the robot actuators fail, they can rearrange themselves using the rest of their body to perform adequately. Accordingly, a non-redundant robot will not have the ability to move properly if one of its joints fail. This property has a great importance in the field of space robotics, since robots cannot be repaired after being launched to space. Some authors have analysed these properties using distributed control architectures [6]. magnetic hyper-redundant robot [1]

Initially, these devices were discrete and made by rigid materials as a logical evolution of traditional ones. However, as time progressed, other variants composed by flexible materials were proposed, so they could continuously bend instead of having distinguishable links. These mechanisms were called continuous robots. Recently, last research efforts have taken a step further creating robots made by soft materials, capable to adapt to their environment and being inherently safe to interact with their surroundings, called soft robots [7].

However, the evolution of discrete hyper-redundant robots to flexible or soft structures does not entail a replacement of the first by the second ones. So to say, they are devised for different applications. For example, continuous and soft robots have higher adaptability skills and are safer to interact with their environment. But they are usually less sturdy, accurate and struggle to carry heavy payloads compared to 
rigid manipulators. Thus, discrete and rigid hyper-redundant robots are better suited for applications in hazardous or adverse environments such as for inspection tasks in hazardous environments. On the other hand, continuous and soft robots are more compliant and exhibit better capabilities to surround delicate objects like fruits or vegetables [8]. In fact, the design of highly-articulated robots is quite challenging since there are multiples parameters that conflict between each other which should be balances.

Once a hyper-redundant robot is designed, it is necessary to extract its behaviour using a reliable and accurate model. This model is expected to describe the robot behaviour under the influence of an actuation system, so their movements can be predicted beforehand. Modelling discrete hyper-redundant robots is a relatively simple task because different values in the actuators can be easily interpolated to obtain displacements and/or rotations in the joints between links. However, continuous and soft robots usually require complex modelling techniques to describe and manage their numerous non-linearities [9]. Latest research also proposes model-free approaches relying on large datasets that collect experimental data from the robot and then used with machine learning algorithms to extract their behaviour [10].

If the behaviour of the robot is reliable and well described, different control strategiesneed to be developed to adequately manage their movements. It is possible to distinguish 3 different levels of control. (i) A lowlevel control based on managing appropriate inputs for the actuators. (ii) When the actuation is not located in the robot body, a mid-level control should study how to transfer the movements from the actuators to the joint space. (iii) Finally, high-level controls rely on previous ones to manage the movements of the whole-body from a higher level of abstraction such as solving the inverse kinematics problem [11], guiding the robot with a follow-the-leader strategy [12], or performing shape controls [13], among many others.

Additionally, since hyper-redundant robots have a large number of degrees of freedom, they often exhibit complex shapes that may frustrate their spatial understanding. Thus, controlling their pose may be difficult using conventional tools. Strategies aimed to adequately define such configurations are usually studied in telerobotics. This discipline links a human operator and a robot in order to execute a remote task. If the information about the remote environment is naturally displayed to the operator, the application will have a high level of telepresence [14]. Since hyper-redundant robots often exhibit complex shapes in the 3D space, maybe immersive technologies can be a good solution to solve this problem [15].

Immersive realities can be understood as those real or simulated environments in which a perceiver experiences telepresence $[16,17]$. They can be classified in 3 categories: (i) Virtual Reality (VR) provides interaction of virtual elements in virtual environments; (ii) Augmented Reality (AR) features interaction of virtual elements in real environments; and (iii) Mixed Reality (MR) offers interaction of real and virtual elements through real or virtual environments [18]. Immersive realities are considered one of the main technologies in Industry 4.0. This revolution aims to combine all the production agents in the shape of cyber-physical systems by means of network connections and information management [19]. Immersive technologies will allow multiple applications such as training of operators, remote and virtual assistance, augmented reality manuals, indoor guidance, immersive 3D design or even teleoperation of robots in remote environments.

The applications derived from a good design, model, control and teleoperation of these robots are almost uncountable. In fact, hyper-redundant continuous and soft robots can be used in multiple scenarios such as in surgical procedures, servicing underground tanks, nuclear core reactors, in the aeronautical and space exploration, for welding activities, inspection of industrial facilities, exploration in mines or for archaeology purposes, deployment of antennas, locomotion purposes, detection and extinction of fires or exploration of coral reefs among many others. 


\subsection{Background}

This section brings together all the concepts necessary to understand the context of this work from scratch. First, an original classification of robots is proposed [7]. Afterwards, this chapter focuses on the main research challenges of hyper-redundant robots. And finally, the appearance of hyper-redundancy in nature has been reviewed.

A robot is a programmable and automatic entity capable to perform intended tasks autonomously without any human intervention, especially the tiring, repetitive or dangerous ones. This term comes from the play R.U.R. (Rossum's Universal Robots) of the Czech writer of the 20th century, Karel Čapek. Robotics is a very multidisciplinary field since it involves areas such as control, mathematics, electronics, mechanics or informatics. A robot can also be identified for either a virtual or a mechanical system, but in this document it will be used in the last sense.

\subsubsection{Robot classification}

It is difficult to establish a precise classification in the robotics scope. This is because the features usually have diffuse boundaries. In previous works, such as [20], certain classification criteria are proposed. However, these ones can be considered incomplete since do not include some types of robots, or do not review the different ways of actuation in depth. The classification proposed below considers: the robot's functionality, the number of DoF, the number of modules, the different types of actuation and their location, and the materials they are made of (Figure 1.2).

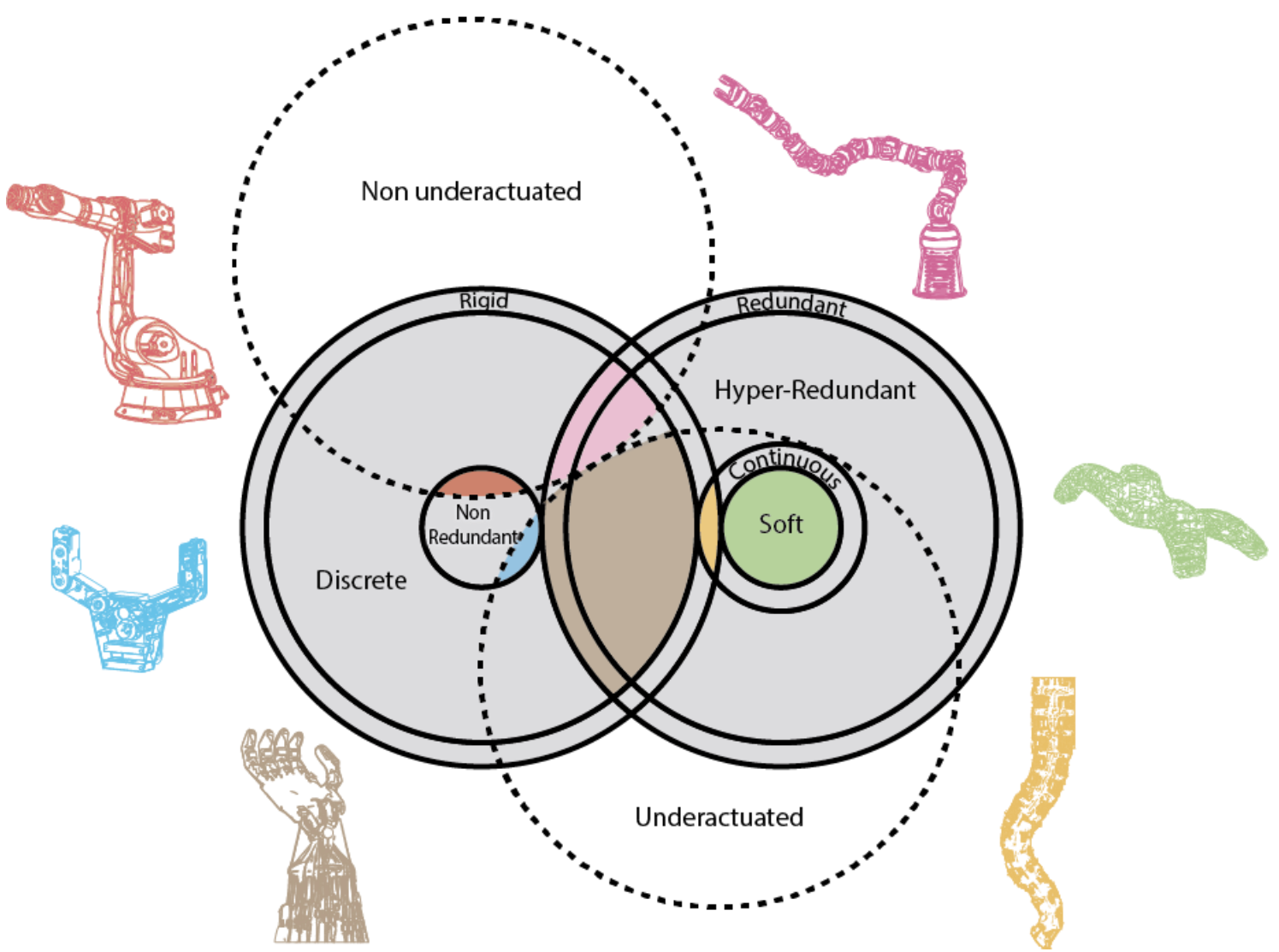

Figure 1.2. Robot classification according to the number of DoF, number of modules, their materials and actuation. Several robotic systems have been used to illustrate this classification [6-11]. The areas in white or grey are empty sets. 


\subsubsection{According to the functionality}

Robots are generally designed for different purposes. Depending on this classification, it is possible to differentiate manipulator, mobile or hybrid hyper-redundant robots. The first ones have a fixed base while their end-effector is capable to move in order to manipulate objects [21]. However, mobile hyper-redundant robots have locomotion capabilities. In other words, skills to move the whole body from one place to another [22]. Sometimes, hyper-redundant manipulators are placed on a mobile base giving rise to hyperredundant mobile manipulators [23]. Accordingly, there is one last category including hybrid ones that presents both manipulative and locomotion capabilities [24].

\subsubsection{According to the number of DoF}

Given a joint space $\bar{q} \in \mathbb{R}^{n}$ that defines the possible positions and orientations of a robot's endeffector, $\bar{x}_{e e} \in \mathbb{R}^{m}$, it can be said that it is necessary at least $n_{p}$ independent parameters to define the position and $n_{o}$ independent parameters to define the orientation of the end-effector. Thus, to position and orient a robot's end-effector properly in a space of dimension $m$, it will be necessary a minimum number of $n_{\min }$ of DoF according to Eq. 1.1 [25]. For example, in a three-dimensional space $(\mathrm{m}=3)$, it would be needed at least 6 DoF to position and orient an end-effector properly.

$$
n_{\min }=n_{p}+n_{o}=m+\frac{m(m-1)}{2}=\frac{m(m+1)}{2}
$$

According to this equation, a manipulator is not redundant if has equal or less DoF than the minimum required to define the position and orientation of its end-effector. Despite non-redundant robots of type $n$ $=\mathrm{m}$ (Eq. 1.2a), robots of type $\mathrm{n}<\mathrm{m}$ (Eq. 1.2a) will not be able to move properly in all the dimensions of their task space. Those robots with more DoF than the minimum necessary can be considered to have a certain level of kinematic redundancy, as specified in Eq. 1.2b. According to this definition, redundant robots will provide some additional DoF to non-redundant ones, extending their abilities to move. Specific literature does not indicate the minimum number of DoF to consider a redundant robot as hyper-redundant [4]. To clarify where that transition can be found, and collecting the terminology used in most of previous works, a mathematical definition is proposed below (Eq. 1.2c):

A robot is kinematically hyper-redundant when has, at least, the double number of degrees of freedom than the minimum required to position and orient its end-effector within a given task-space.

$\begin{array}{lll}\text { Non-redundant: } & (n=\operatorname{dim}(\bar{q})) \leq\left(\operatorname{dim}\left(\bar{x}_{e e}\right)=m\right) & \text { Eq.1.2a } \\ \text { Redundant: } & (n=\operatorname{dim}(\bar{q}))>\left(\operatorname{dim}\left(\bar{x}_{e e}\right)=m\right) & \text { Eq. 1.2b } \\ \text { Hyper-redundant: } & (n=\operatorname{dim}(\bar{q})) \geq 2\left(\operatorname{dim}\left(\bar{x}_{e e}\right)=m\right) & \text { Eq. 1.2c }\end{array}$

The justification of this expression that defines hyper-redundant robots is also double. On one hand, as said before, hyper-redundant robots have greater kinematic capabilities. One of them is the capacity to enclose an object with its own body. A non-redundant robot of type $\mathrm{n}<\mathrm{m}$, according to Eq. 1.2a will not be able to wrap any object using its own body (Figure 1.3a). However, a non-redundant robot of type $n=m$, attending to Eq. 1.2a, has the minimum number of DoF to position and orient its end-effector properly. Theoretically, its end-effector will be able to reach the base, being able to enclose an object, although the manipulability 
will be reduced (Figure 1.3b). As the number of DoF increases, the robot will be redundant according to Eq. $1.2 \mathrm{~b}$. Then, it will be able to surround an object with a greater manipulability, while still unable to position and orient that object properly (Figure 1.3c). Only a hyper-redundant robot, according to Eq. 1.2c will be able to grab an object with its own body while also being able to move it appropriately within its task space (Figure 1.3d).

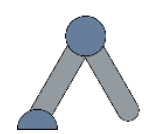

(a)

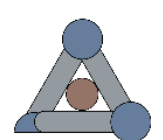

(b)

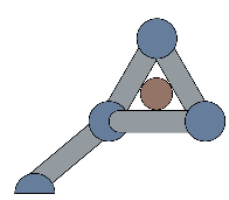

(c)

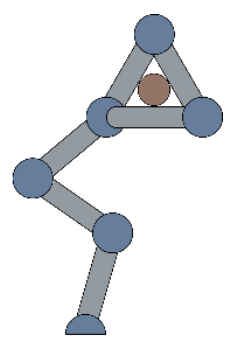

(d)

Figure 1.3. Front view of different types of robots with rotational joints in a three-dimensional space ( $m=3$ ). (a) Non-redundant robot type $n<m$. (b) Non-redundant robot type $n=m$. (c) Redundant robot Type $n>m$. (d) Hyper-redundant robot type $n=2 m$.

Secondly, it can be reminded that hyper-redundant robots are fault tolerant by definition. If a robot has a higher number of DoF than in Eq. 1.1, then it will be able to handle a failure in some of their joints. However, if the number of non-functional DoF exceeds the number of redundant DoF, then the robot will loose some degree of functionality, becoming a robot type $\mathrm{n}<\mathrm{m}$ according to Eq. 1.2a. A hyper-redundant robot should be more fault-tolerant than a redundant one. Therefore, it should be able to perform properly even after the failure in as many DoF as those in the task space.

Because of these reasons, in a two-dimensional space, a robot will be considered non-redundant if it has up to 3 DoF, redundant if it has more than 3 DoF and hyper-redundant if it has 6 DoF or more. In the same way, in a three-dimensional space, a non-redundant robot will have up to 6 DoF, a redundant robot more than 6 DoF and a hyper-redundant robot 12 DoF or more. In Table 1.1 a comparison of some properties and capabilities of these robots can be found following the previous classification of Figure 1.2.

\begin{tabular}{|c|c|c|c|c|c|c|}
\hline & $\begin{array}{l}\text { Non- } \\
\text { redundant }\end{array}$ & $\begin{array}{l}\text { Non-redundant } \\
\text { and } \\
\text { underactuated }\end{array}$ & $\begin{array}{l}\text { Hyper- } \\
\text { redundant } \\
\text { discrete }\end{array}$ & $\begin{array}{l}\text { Hyper- } \\
\text { redundant } \\
\text { discrete and } \\
\text { underacted }\end{array}$ & Continuous & Soft \\
\hline \multicolumn{7}{|l|}{ Properties } \\
\hline DoF, $n$ & $n \leq m$ & $n<m$ & $n \geq 2 m$ & $N \geq 2 m$ & $n=\infty$ & $n=\infty$ \\
\hline Actuators, $a$ & $a=n$ & $a<n$ & $a=n$ & $a<n$ & $a<<n$ & $a<<n$ \\
\hline Material & Rigid & Rigid & Rigid & Rigid & Rigid & Soft \\
\hline \multicolumn{7}{|l|}{ Capabilities } \\
\hline Precision & 00000 & 00000 & 00000 & 00000 & 00000 & 00000 \\
\hline Adaptability & ○ 0000 & 00000 & $\bigcirc \bigcirc \circ \circ \circ$ & 00000 & 00000 & 00000 \\
\hline Cycle time & $0 \bigcirc \bigcirc \bigcirc \bigcirc$ & 00000 & 00000 & 000000 & $0 \bigcirc 0 \circ \circ$ & 00000 \\
\hline Load capacity & 00000 & 00000 & 00000 & 00000 & $0 \bigcirc 0 \circ \circ$ & 00000 \\
\hline Safe interaction & 00000 & 00000 & $0 \circ 0 \circ \circ$ & $000 \circ \circ$ & 00000 & 00000 \\
\hline
\end{tabular}

Table 1.1: Properties and capacities of the different types of hyper-redundant robots

Additionally, the concept of hyper-redundancy has been extended to systems that can actively change their number of degrees of freedom, also called growing robots. Growing usually refers to an increment in size and/or mass of an organism. It is often irreversible and can be considered as a form of response in form of morphological adaptation to environmental changes [26]. Growing robots are often inspired by natural 
systems, like plants [27], vines [28], roots [29], cells like neurons [30], and invertebrates, such as annelids [31] or arborescent animals [32].

In this work, growing robots will be defined as programmable devices that are able to modify their number of degrees of freedom. As a consequence, they can increase their maneuverability as they grow and adapt to new tasks. These capabilities are often achieved by eversion or addition of matter to their structure [33]. In addition, they are often made by soft materials to further increase their flexibility [7].

Hyper-redundant and growing robots have a lot of applications such as assistance for surgical procedures, like endoscopies or colonoscopies [34], deployment of antennas [35], for locomotion [26], like guidance of cables through underground or undersea pipes, monitoring and local treatment of water in contaminated rivers, detection and extinction of fires [33], exploration of coral reefs [36], or inspection through small and long cavities in space exploration, aerospace, industrial equipment, mines or archaeology [28].

\subsubsection{According to the number of modules}

In the previous Section 1.2.1.2, hyper-redundant robots have been classified according to the number of DoF. It is important to differentiate the concept of joints from the number of DoF. A joint is a union between two elements that allows a relative movement between them. Thus, a joint can be provided by one or more DoF. According to the number of modules, it is possible to distinguish between discrete robots and continuous robots [20,37-39].

The first ones are mechanisms built by a series of rigid links distinguishable from each other (Figure 1.2) [40]. However, continuous robots are not formed by identifiable links, but by structures that are continuously deformed due to elastic properties [41]. In this type of robots, a single actuator can cause movements around several or infinite axes. Because of this, some authors affirm that continuous robots have virtual DoF. As explained below, all continuous robots will be considered as underacted. Also, it is worth noting that not all continuous robots are soft but also rigid. However, as further explained, all soft robots will be considered continuous because of their inherent properties (Figure 1.2).

Other authors include in the classification serpentine hyper-redundant robots [42]. This is, in other words, a type of discrete manipulator with a high number of short and rigid links. It has not been included in the classification, but it could be considered to belong near the tangency point between the set of discrete and continuous robots (Figure 1.2).

\subsubsection{According to the actuation}

An actuator is a device capable of transforming energy into movements responding to the orders given by a control unit. Some of the most used actuators in hyper-redundant robotics are: motors [43], hydraulic actuators [44], shape memory alloys (SMA) [45], piezoelectric [46], artificial muscles [47] or pneumatic actuators [48]. It is possible to find a comparison of these forms of actuation in [49].

Depending on the number of actuators, it is possible to distinguish between non-underactuated and underactuated robots. Be $\bar{w} \in \mathrm{R}^{\mathrm{a}}$ the space that defines the actuation, a robot will be considered as nonunderactuated if each of its actuators is responsible for the movement of a single DoF according to Eq. 1.3a (Table 1.1). However, underactuated robots usually have a smaller number of actuators than DoF. Therefore, there may be a single actuator responsible for the movement of several virtual-DoF as defined in Eq. 1.3b (Table 1.1). Formally, underactuated robots will be those mechanical systems that cannot be 
controlled to follow an arbitrary trajectory in the configuration space ${ }^{2}$ [50]. Because of this, robots previously defined as continuous are necessarily underactuated since they have an infinite number of DoF, and it is not physically possible to control all of them by an infinite number of actuators. In the same way, it is also possible to find redundancy in the number of actuators, commonly in parallel robots [51].

$$
\begin{array}{lll}
\text { Non-underacted: } & (a=\operatorname{dim}(\bar{w}))=(\operatorname{dim}(\bar{q})=n) & \text { Eq. } 1.3 a \\
\text { Underactuated: } & (a=\operatorname{dim}(\bar{w}))<(\operatorname{dim}(\bar{q})=n) & \text { Eq. } 1.3 b
\end{array}
$$

Depending on the location of the actuators with respect to the controlled DoF, it is possible to distinguish between intrinsic or distributed actuation, when it is located within the robot mechanism itself, or either extrinsic or remote, in those cases where the movement is transferred from an external location. A third type of actuation called hybrid can be considered as well in those cases that combine the two previous types of actuation [42].

An intrinsically actuated robot is usually more accurate, but heavier. The weight of the robot is very important in the robotics in general, and even more in hyper-redundant robotics because they are usually longer than conventional ones. A robot with extrinsic actuation is lighter and easier to miniaturize, but it may exhibit difficulties in their control or portability.

\subsubsection{According to their materials}

Depending on the materials the robots are made of, they can be defined as rigid or soft [52, 53]. Although, like previous classifications, it is possible to find semi-rigid or semisoft robots in areas near to the tangency point of both rigid and soft sets (Figure 1.2). In engineering, rigidity is a property of a material, component or structural system that characterizes the difficulty of deforming it. However, in practice, it is said that a robot is rigid or soft based on their adaptability with their environment and their security when colliding.

According to some authors [54], the Young's modulus (E) could be a useful parameter, but limited, to measure the adaptability of a robot with its environment. According to this work, rigid robots could have materials with a higher Young's modulus than $10^{9} \mathrm{~N} / \mathrm{m}^{2}$ and soft robots in between $10^{2}$ and $10^{6} \mathrm{~N} / \mathrm{m}^{2}$, approximately.

However, this parameter only gives information of the elongation of prismatic and homogeneous beams subject to small deformations through an axial load. On the other hand, energy absorbed by a material during an impact is usually measured by the Shore hardness scale, so it gives an idea of the elasticity. This value is dimensionless and, usually, three overlapped scales are used to quantify its value: Shore 00, Shore $A$ and Shore D. Attending to Shore 00, a material is very soft or soft if it has up to 75, semisoft if it has from 75 to 90 or rigid if it has from 90 to 100 . To measure the elasticity of rigid or very rigid materials it will be necessary to use the scales Shore A or Shore $D$, respectively. According to Shore $D$, a material is rigid if it has from 30 to 60 and very rigid if it is higher than 60. It should be noted that there is a proportionally linear relationship between the Young's modulus and the Shore hardness scale [55]. Stiffness or elasticity can give information towards the adaptability of a robot with its environment, but do not provide much information about their security when interacting with objects.

Robot safety can be understood as the damage that could happen because of an unwanted collision with a human or other object. There are many works that study these situations [56-58]. In this context, rigidity should be studied alongside with the effective inertia of the robot. In this way, the Head Injury Criterion

${ }^{2}$ Configuration space: It is an alternative to the task space that represents an environment with obstacles depending on the joint values of a robot that aims to avoid them. Therefore, the configuration space, or C-space, will have a dimension equal to the number of DoF of the robot. 
(HIC) can be useful to measure the damage as a result of an impact. So, to consider a robot as soft, it must gather both the rigidity of the materials and the inertia produced by their movements. Related to this, it is worth highlighting also that morphologies can be designed to increase the safety as part of the design process. For example, it is frequent to use folds in the structure of soft robots to provide a macroscopic elastic behaviour according to the Hooke's law [48].

Rigid robots are usually more accurate and carry heavier loads than soft robots. However, they are less versatile and less safe according to a possible collision. On the other hand, soft robots interact in delicate environments, such as the human body, in a harmless and compliant way. In addition, they can be constantly deformed, so they should be considered as a type of continuous robots. However, not all continuous robots can be considered as soft (Figure 1.2).

\subsubsection{Challenges in hyper-redundant robots}

The hyper-redundant robotics field has a lot of potential, but the application of these robots are often limited to the research field. Figure 1.4 shows the evolution of different types of hyper-redundant robots in the scientific field over the past recent years. It is worth highlighting that the impact of continuous and, specially, soft robots has increased exponentially in the research scope against discrete, hyper-redundant ones.

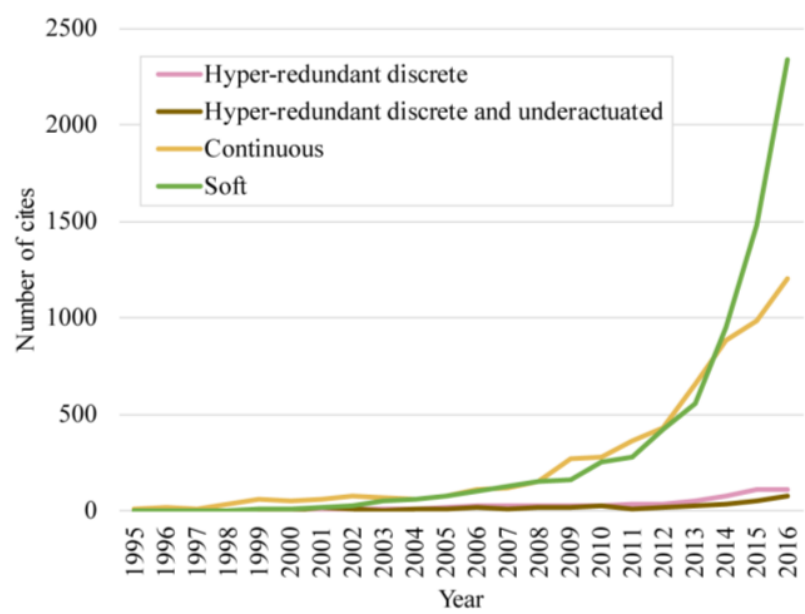

Figure 1.4. Research impact of the types of hyper-redundant robots in recent years. Source: Web of Science.

Current challenges within the research field to develop hyper-redundant robots will be explained below. These problems are classified in four categories: provide new designs and sensing capabilities, find reliable models capable to extract their complex behaviour, study new types of control and motion planning, and developing updated teleoperation architectures to manage their movements from remote and safe locations.

\subsubsection{Design and sensors}

The mechanical design of hyper-redundant robots is inherently complex. Usually, it consists on the design of a module that is sequentially repeated throughout its structure [59]. This strategy allows the creation of modular hyper-redundant systems, which are reconfigurable and easier to repair in case of failure. However, these robots are strongly conditioned by the type of actuation and their location.

In the case of continuous robots, it is important to appropriately select the elastic structural elements that will provide their movement properties. Because of their nature, robust materials should be used with long life-time functionalities. In turn, concatenating multiple continuous sections allow adjusting their rigidity 
depending on the objective. On the other hand, it is not always easy to define actuation limits to prevent collisions [60].

In the specific soft-robot literature, the variable-stiffness terminology is commonly used. This strategy is often used for soft robots that rely on hydraulic or pneumatic continuous actuators. These systems allow the dynamic adjustment of the rigidity of the structure to perform fast movements, or firmly surround an object with the robot body. The design of these robots is strongly coupled with their morphology, actuation and weight. For this reason, it is not easy to define the type of tool for the end-effector due to the added weight, which occasionally may be compensated by complex control strategies.

In addition, the feedback concept is also fundamental in robotics and automation. A feedback system applied to a robotic device allows to make precise controls in a closed loop from a prior-knowledge of its behaviour. For this purpose, the use of sensors that provide information of the current state of the robot is essential. Consequently, it is important to note that in hyper-redundant robots, the knowledge of the end-effector position and orientation is as important as the pose of the whole-body itself.

Encoders or traditional pulse generators often lack information to reconstruct the configuration of any underactuated robot. For this reason, many works have focused on alternative sensors such as external cameras and computer vision [61]. However, such methods are only feasible in highly controlled environments and, therefore, present low portability. In other projects, the use of inertial measuring units (IMU) has been proposed to solve this problem [62]. Additionally, more advanced sensory systems embedded on the robot structure are able to extract the curve that models its configuration [63]. These sensors are based on the Fiber Bragg Grating (FBG) [64] or Fiber Optic Shape Sensing (FOSS) [65] technologies, which features high precisions for non-extensible devices, low portability and high costs. Sensors are also used to measure the intrinsic strength capabilities of the robot, or for calibration purposes, allowing further adjustment of their rigidity or length $[66,67]$.

Hyper-redundant robots are composed by a high number of joints. For this reason, their production process is often difficult and laborious as well. Moreover, a large number of joints usually also involves a high number of actuators, sensors, transmission elements, controllers and other elements that raise the economic cost. Soft robots, in this sense, are an exception since they can usually be rapidly produced by moulding with low costs $[48,68]$. An up-to-date state of the art that collects past efforts of hyper-redundant, continuous and soft robots will be explained in Section 2.1 and some original designs will be presented in Section 3.

\subsubsection{Modelling}

Robot modelling allows the theoretical extraction of its behaviour within a given environment or application, in order to predict its movements and apply precise control actions. Rigid hyper-redundant robots do not present major problems in this context since classical techniques of conventional robot modelling can be extrapolated for a greater number of joints.

However, continuous and soft robots frequently rely on their elastic properties, exhibiting a high number of non-linearities that hinder their modelling extraction. In this respect, it is possible to distinguish two main different forms of modelling: model-based and learning-based.

Model-based approaches consists on creating a user-defined model, which can be either analytical or numerical, but accessible with no need of a training set. This approach can be subdivided into 2 main categories: kinematic and dynamic models. 
Kinematic models study the robot movements assuming the forces involved in the system are negligible. According to some authors, models that approximate continuous manipulators using discrete hyperredundant kinematics may also be appropriate $[11,20]$. Others, provide models for continuous robots based on constant curvature approximations, usually providing lower precisions [60]. Kinematic models often lack precision to enable reliable controls.

On the other hand, dynamic models study forces involved in the robot movements. In this context, some model techniques focus in theoretical basis such as elasticity and energy minimization [69] or Cosserat Rod theory [70]. These models have been particularly efficient including the effects of gravity, torsions and load capacities against previous methods. However, they cannot study the interactions of these robots with their environment. In addition, it is worth mentioning other approaches based on real-time Finite Element method (FEM) to promise good generalization for soft robots [9].

Another modelling strategy is often called as learning-based or model-free since they rely on machine learning to obtain the robot's behaviour by using large datasets. Related to this, some of the main attempts are based on Artificial Neural Networks (ANN) [71] and Fuzzy models[72].

Extraction of models in continuous and soft robots still requires further study to obtain a generalizable method, computable in real-time and precise enough to take reliable control actions for all kinds of continuous and soft robots. These methods will be further discussed in Section 4.

\subsubsection{Control and motion planning}

Highly articulated mechanisms are by definition underdetermined systems in terms of their kinematic control. Thus, they provide multiple solutions that satisfy the same problem. This is a huge advantage against traditional, non-redundant robots but it is also a great challenge in terms of finding the mostadequate solution. The optimality to manage the movements of these robots will rely on the context of the robot, the tasks and the surroundings.

Another field of great importance in hyper-redundant robotics is motion planning. This problem aims to find the movements of a robot in an environment with obstacles with the purpose of avoiding any possible collision. Traditional techniques usually make use of tools such as the $C$-space, also known as joint or configuration space $[73,74]$.

However, in the case of hyper-redundant robots, the joint space can be quite large. The computational cost of such methods is unviable. For this reason, the motion planning problem of discrete hyper-redundant robots has been oriented through other techniques such as Voronoi Diagrams [75], using geometric methods [76], algorithms based on Rapidly-exploring Random Trees (RRT) [77], or by Particle Swarm Optimization $[78,79]$ among others.

Motion planning techniques for continuous robots suffer from great limitations. There are several examples of work in this area, with a special emphasis on the medical field, but often rely on high computational costs hindering real-time applications [80].

Probabilistic methods usually also have high computational costs because require the generation of points in the space of the configuration, which grows exponentially with the number of DoF. Besides, state-of-theart methods usually do not consider restrictions on the physical properties of hyper-redundant robots. Therefore, most of motion planners tend to work for very specific cases and not much advances have been made for flexible robots in general. Some of the most used planners for soft are the Probabilistic Roadmaps (PRM) [81] or others such genetic algorithms [82], among others. Control and motion planning challenges will be further analysed in Section 5. 


\subsubsection{Teleoperation}

Classical techniques used to solve the kinematic modelling of conventional robots have not been efficient or well-adapted to the needs of hyper-redundant robots. Forward kinematics ${ }^{3}$ of these robots does not have a great complication but solving the Inverse kinematics ${ }^{4}$ is not as easy as it may seem. In fact, a hyperredundant manipulator can achieve a certain location - position and orientation - with its end-effector by a large or an infinite number of different joint configurations [84]. And choosing one solution among all of them can be a big challenge. For instance, to solve this issue for discrete hyper-redundant robots, some authors have proposed techniques such as the Jacobian pseudoinverse matrix [59], pattern search, global search, genetic algorithms, simulated annealing or neural networks [85], among others.

In the case of continuous robots, and particularly for soft robots, the kinematic modelling is complicated because both have a virtually-infinite number of DoF. In these cases, a precise control requires an elastic model-based prediction to accurately describe their shape from a macroscopic scale. Simpler model-based controls are not expected to guarantee great stability or effectiveness [86].

However, in hyper-redundant robotics, the paradigm changes. A lot of works focus on finding the optimal solution to solve the inverse kinematics problem. The meaning of optimality here relies on the robot configuration itself. Thus, finding the joint values that locates the end effector is as important as finding the joint values that locate the whole-body itself. Consequently, the user is usually not only expected to control the hyper-redundant robot end-effector, but the whole body. By doing this, single inputs are not needed but multiple-dimension ones instead. This kind of practices are usually studied in teleoperation ${ }^{5}$. And there is a lack of interfaces optimized for teleoperating such robots in previous works. All of this will be analysed in Section 6 .

\subsubsection{Biomimicry}

The terms biomimetics and biomimicry derive from Ancient Greek: bios - life - and mimesis -imitation - . Thus, they can be understood as the imitation of the models, systems, and elements of nature for the purpose of solving complex human problems [88].

Live organisms have evolved to become very well-adapted structures and materials through natural selection. In this context of a large lapse of time, now it is possible to find extremely intelligent designs that can be taken advantage of, by imitating or modifying its structures or behaviours. Biomimetics is not intended to supersede the nature with better designs, but to take those beneficial aspects with potential interest for a specific end. For example, termites' skills to virtually maintain constant the temperature in their nests are inspired to save in air conditioning [89], gecko adhesion, bat sonar, squirrel hibernation, spider and fly vision, lizard limb regeneration, chemo-sensing in moths, spider web materials or flexibility and strength in octopus and squid arms [90].

Relating to the latter, in the hyper-redundant, continuous and soft robotics scope, these devices are often inspired by biomechanical models such as snakes [91], squid tentacles [92], octopuses [93,94] or elephant trunks [95], among others.

\footnotetext{
${ }^{3}$ Forward kinematics refers to the use of the kinematic equations of a robot to compute the position of the end-effector from specified values for the joint parameters 83.Paul, R.P., Robot manipulators: mathematics, programming, and control: the computer control of robot manipulators. 1981: Richard Paul.. ${ }^{4}$ Inverse kinematics is the mathematical process that makes use of the kinematics equations to determine the joint parameters that provide a desired position for each of the robot's end-effectors 83.Ibid..

5 Telerobotic systems allow human operators to properly interact with a robot to manipulate objects located in a remote environment 87.Sheridan, T.B., Telerobotics, automation, and human supervisory control. 1992: MIT press..
} 


\subsection{Objectives}

The pursued goals in this thesis are organized as follows:

- This work aims to study highly-articulated devices in a generalizable perspective. Thus, their definitions and classifications needed to be updated as showed in this chapter, splitting the diffuse borderlines that have traditionally hindered their comprehension. Also, their current challenges needed to be analysed to focus the efforts of this work.

- Also, it is required to present an exhaustive state-of-the-art to configure an outlook of the problematics that hinder the development of hyper-redundant robots. Therefore, next objectives will be focused on solving these challenges, explained as follows.

- Past designs of hyper-redundant robots need to be further analysed in this work to propose new structures, ways of actuation or sensors to improve their mechanical limitations and enhance their performance.

- The modelling issue is approached from different perspectives, but often lack precision, speed or interaction features, essential to take reliable control actions over all types of hyper-redundant robots such as those discrete, continuous or soft ones. Thus, this work should address this challenge and implement feasible modelling strategies.

- Traditional methods to control hyper-redundant robots have been implemented from the basis of non-redundant ones. Hence, they are not always well-adapted to their capabilities. Thus, this work should propose new solutions to manage the movements of such robots.

- Once the hyper-redundant robots are analysed, designed, modelled, and controlled, further humanrobot interfaces should be proposed to effectively manage their movements in a simple and transparent way.

- The repercussion of this work should be properly analysed to guarantee a good practice of the scientific outcome from this thesis.

It can be deduced that this thesis is very transversal since it is intended to provide original contributions about multiple areas of knowledge in hyper-redundant, continuous and soft robotics. 


\subsection{Organization of this work}

This work is structured as follows:

- Section 1 has reviewed robotics, defined hyper-redundant robots from an original perspective, presented a new and inclusive classification of such robots and deepened in their challenges to focus in the objectives of this work.

- Section 2 examines the up-to-date efforts related to the design, modelling, teleoperation and control of hyper-redundant robots.

- Section 3 presents original designs for hyper-redundant, continuous and soft robots created in the framework of this thesis to enable the study of their main advantages and limitations, serve as testbeds to implement algorithms and propose new actuation and sensory systems.

- Section 4 considers the modelling issue as the base to predict the robot movements and enable further control actions. Thus, modelling strategies will be implemented and explained either for discrete, continuous and soft robots.

- Section 5 determines the control approaches derived from the previous developments to perform automatic tasks for hyper-redundant robots.

- Section 6 analyses the application of immersive technologies such as virtual or augmented reality as original interfaces to manage the movements of hyper-redundant robots from remote and safe locations.

- Section 7 studies the repercussion of this work in terms of their applications and benefits, sustainability and ethics and other achievements.

- Section 8 outlines the contributions, the conclusions and the future research lines derived from this work.

- Section 9 collects the references that this research uses to support a reliable scientific praxis. 



\subsection{Design}

This section will present a review of the most representative hyper-redundant robots throughout their history [7]. To facilitate the understanding, some of the most relevant hyper-redundant robots will be presented to illustrate the features of each subfield mentioned in Section 1.2.1.

\begin{tabular}{|c|c|c|c|c|c|c|}
\hline Name & Classification & DoF & Sections & Actuation & Material & Applications \\
\hline Woodstock [40] & Discrete & 12 & 6 & 12 intrinsic motors & Metal & Search and rescue \\
\hline Series II X125 [96] & Discrete & 24 & 12 & $>30$ extrinsic motors & Metal & Welding \\
\hline Elephant Trunk [21] & $\begin{array}{l}\text { Discrete and } \\
\text { underactuated }\end{array}$ & 32 & 4 & 8 extrinsic motors & - & Research \\
\hline Tendril [41] & Continuous & $\infty$ & 2 & 4 extrinsic motors & Metal & Spatial missions \\
\hline ET Arm [97] & Continuous & $\infty$ & 2 & $\begin{array}{l}6 \text { extrinsic motors and } \\
6 \text { pneumatic muscles }\end{array}$ & - & Research \\
\hline Air-Octor [99] & Soft & $\infty$ & 2 & $\begin{array}{l}3 \text { extrinsic motors and } 1 \\
\text { pneumatic actuator }\end{array}$ & - & Research \\
\hline OctArm $V[100]$ & Soft & $\infty$ & 3 & 9 pneumatic muscle & - & Search and rescue \\
\hline$B H A[101]$ & Soft & $\infty$ & 3 & 9 pneumatic actuators & Polyamide & Cooperative tasks \\
\hline$[102]$ & Soft & $\infty$ & 1 & 2 extrinsic motors & Silicone & Research \\
\hline
\end{tabular}

Table 2.1. Most representative models of hyper-redundant manipulators in the state of the art.

\subsubsection{Discrete and mobile hyper-redundant robots}

This section will briefly explain the most representative models of hyper-redundant robots, in other words, those with locomotion capacities. One of the first robots of this genre was presented in 1972 by Hirose and called ACM III (Active Cord Mechanism). This robot with 20 DoF, actuated by servomotors, could move undulating, similarly to snakes, using a set of passive wheels to minimize the friction with the ground [103].

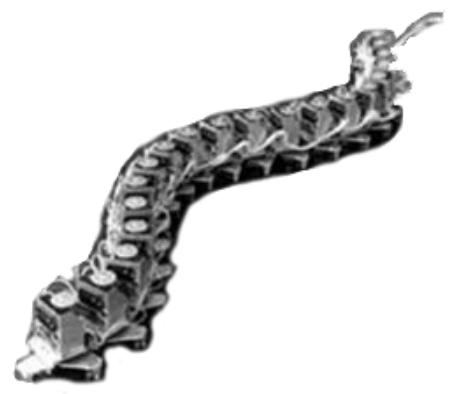

Figure 2.1. ACM III, one of the first hyper-redundant robots made in 1972 by Hirose [103]

Since then, many other models of mobile hyper-redundant robots were developed, attempting to improve the Hirose's model. Some of them , used active wheels like the GMD Snake [104], GMD Snake 2 [105], MAKROPIus [106] or Genbu [107]. Others chose to use continuous track, as the case of Moira [108], Kohga [109], Soryu [110] or OmniTread [111]. 


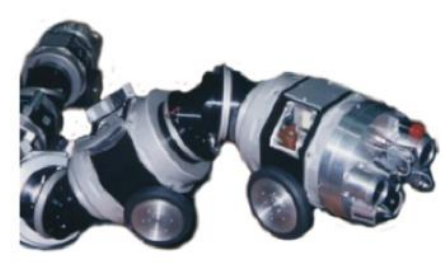

(a)

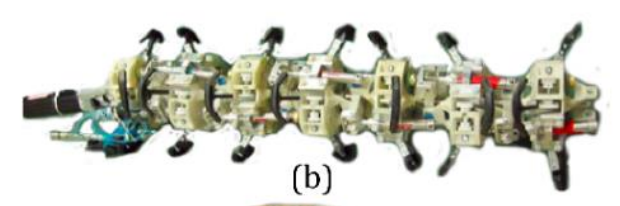

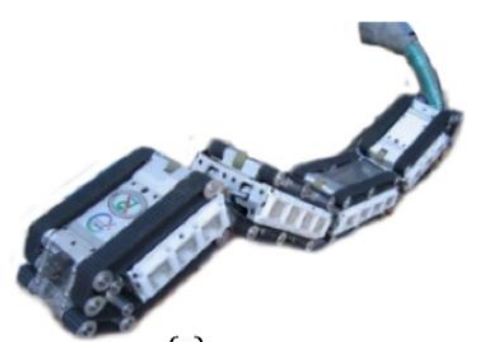

(c)

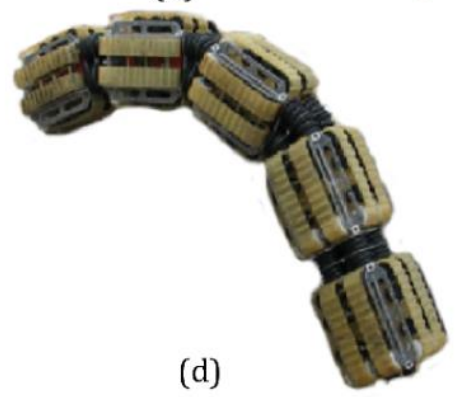

(d)

Figure 2.2. Relevant mobile hyper-redundant robots. (a) MAKROPlus [106], (b) Genbu [107], (c) Moira [108] and (d) OmniTread [111].

Afterwards, the locomotion was performed without using traction elements in robots such as Amphibot I [91], Amphibot II [112], Anna Konda [113], Slim Slime [114], Kulko [115] or Unified Snake Robot [24]. Other line of development focused on the integration of legs to improve the movement through unstructured terrain, as shown in OmniPede [116]. Later, the ACM III evolved to an industrial version: the ACM R5, provided with active wheels and flippers to swim and dive in underwater environments [22].

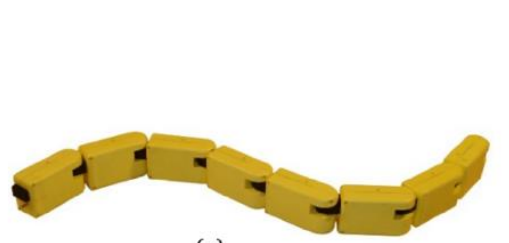

(a)

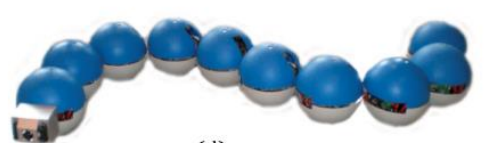

(d)

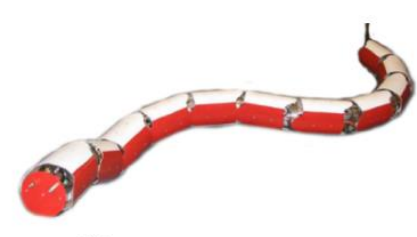

(b)

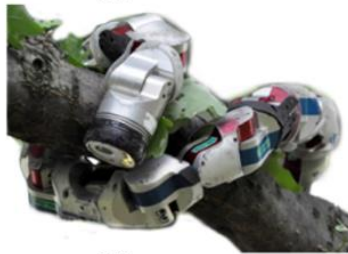

(e)

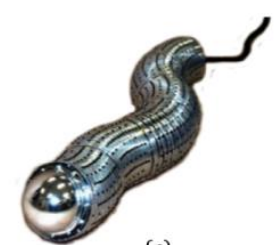

(c)

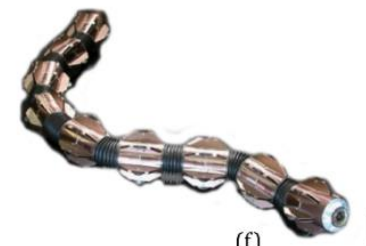

(f)

Figure 2.3. Relevant mobile hyper-redundant robots. (a) Amphibot II [112], (b) Anna Konda [113], (c) Slim Slime [114], (d) Kulko [115], (e) and (f) ACM R5 [22].

Additional information about the state of the art of mobile hyper-redundant robots can be found in [117119].

\subsubsection{Discrete hyper-redundant manipulator robots}

Discrete hyper-redundant manipulators have been often inspired by previously explained mobile models. This is the case of Woodstock [40] (Table 2.1), a 12 DoF robot actuated by 6 universal joints, that stands out for its high load capacity and compact design. These are the features that stand out the most compared to 
previous robots of its genre, like NEC [120] or JPL Serpentine Robot [121]. In fact, Woodstock is a mobile hyper-redundant manipulator. Its control is achieved remotely with a wireless network and each joint has a rotation range of $\pm 50^{\circ}$, so it has an accumulated maximum rotation of $300^{\circ}$.

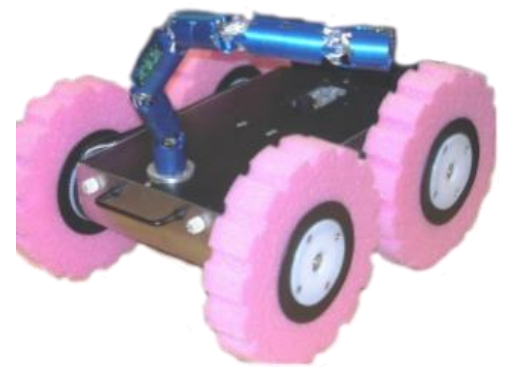

Figure 2.4. Woodstock, a very compact and strong robot [40]

Traditionally, discrete hyper-redundant robots have been the most used since their beginnings. However, current tendencies in the research of this type of robots are evolving to the study of continuous and soft robots. Some examples are the robots of the company OC Robotics [122]. Their style is usually called as continuous, however they are not strictly continuous, but composed of a very high number of rigid links connected in series, allowing their configuration to look-like almost continuous [123]. For instance, the model Series II X125 presents 10 joints, 22 DoF, a load capacity of $5 \mathrm{~kg}$, and has an accumulative bending angle of $225^{\circ}$. In addition, this robot features advanced controls that allow welding operations [96] (Table 2.1).

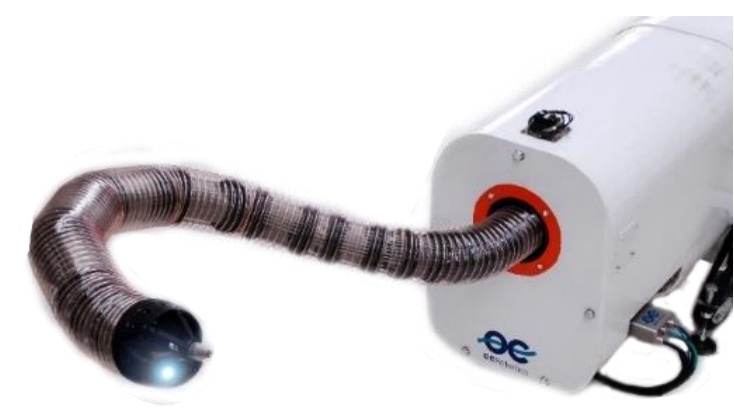

Figure 2.5. Series II X125, one of the few and most advanced industrial hyper-redundant robots.

\subsubsection{Continuous robots}

In the late 20th century, Gregory S. Chirikjian, researcher at Johns Hopkins University, made fundamental contributions in the development of continuous manipulator robots. He proposed a modelling strategy for this type of robots by approximating their shape to a curve $[38,124]$. This methodology was checked in a hyper-redundant manipulator of $30 \mathrm{DoF}$, in which also studied obstacles avoidance, various forms of locomotion and some gripping algorithms [125].

Later, at the beginning of the 21st century, there were numerous advances in the design and modelling of this type of robots thanks to the work of Walker and his research group, from Clemson University, which proposed a manipulator inspired by an elephant's trunk [21] (Table 2.1). He also studied the theoretical foundations of continuous robots [126], and proposed a model to approximate them assuming that their sections are constantly curved [52]. This robot can also be considered as hyper-redundant discrete and underacted. 


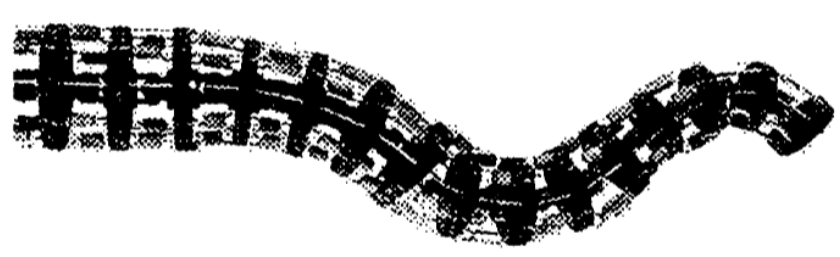

Figure 2.6. Elephant's trunk by Hannan and Walker [21].

Continuous robots offer multiple advantages against other such as the ability to surround objects with their whole body. However, continuous robots usually present complex controls. For this reason, Walker argued to combine continuous elements - to adapt with the environment - and rigid ones - to achieve more accurate positioning - [127].

One of the fields in which continuous robots have greater impact is in medicine. For example, for minimally invasive surgeries [34, 128-131], endoscopies [46, 132, 133], or colonoscopies [134]. In recent years, many miniaturization efforts for this type of robots have been achieved $[67,135]$.

Another example of a continuous robot with great impact on the research field is Tendrilmade by NASA. It is a manipulator robot designed for minimally invasive tasks in space missions. Its peculiarity is based on an eccentric morphology with great length and small diameter compared to other models made up to that time. The greatest inspiration for its design comes from those made for medical applications, although such models did not reach similar lengths. It consists of two joints provided with springs to make prismatic movements, and a set of cables that allow their rotation. The springs provided some consistency to maintain the robot structure and were also used to control its compression. The cables were connected using two motors and pulleys for each joint, so that the retraction of a tendon enabled the extension through the other end [41] (Table 2.1).

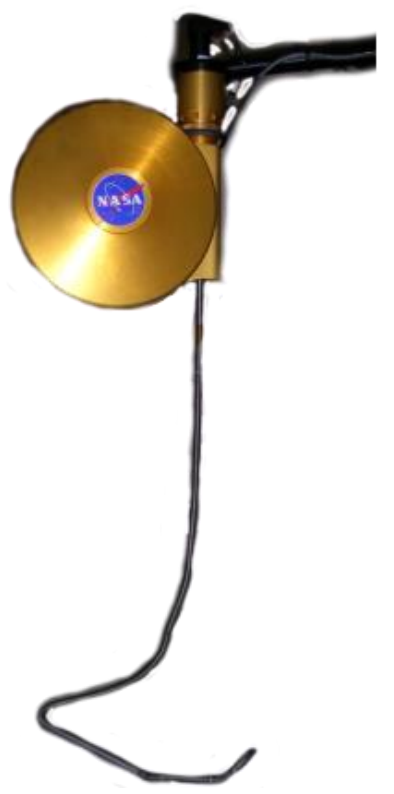

Figure 2.7. Tendril, made by NASA to perform minimally invasive tasks in space [41].

Over the years, hybrid systems were also studied, either because the actuation strategy such as the case of ET Arm - using both pneumatic actuation and tendons - [97], or either because the materials - partially soft and rigid - $[136,137]$. This methodology comes from the idea of Walker of finding a balance between 
properties of different types of robots. For example, the ET Arm (Elephant Trunk Arm) was designed in order to present a new system of actuation not composed either by cables or by pneumatic actuators. It was made up of two segments, each one provided with multiple elastic structures, an artificial skin and 3 artificial muscles. Besides, it was actuated by a series of motors arranged in its base, allowing the continuous movement of its structure [138] (Table 2.1).

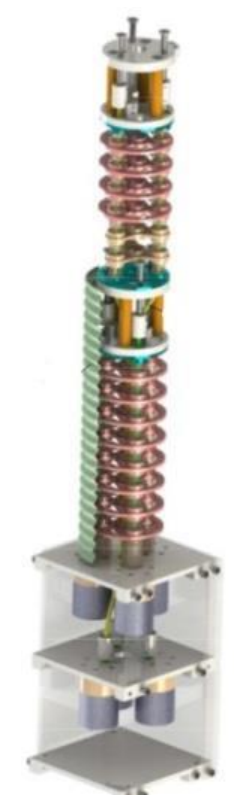

Figure 2.8. ET ARM, actuated by artificial muscles [138].

Moreover, other continuous robots were designed without the use of elements as springs within the structure, such as the robot of the University of Nottingham. It was conceived inspection and reparation tasks in wind turbines. It is a very flexible model and, just like Tendril, it has a high eccentricity regarding the length-to-width ratio. It has $25 \mathrm{DoF}$ and is capable to navigate in unstructured environments. Also, it has a camera on its end-effector to allow in-situ interventions. Additionally, it presents a very compact and innovative mechanical structure, which makes use of pairs of cables to minimize the number of actuators; as well as pairs of joints, to allow high accumulative bending angles. To achieve the maintenance tasks, a set of innovative control algorithms allows its navigation and generates the end-effector trajectory [98] (Table 2.1).

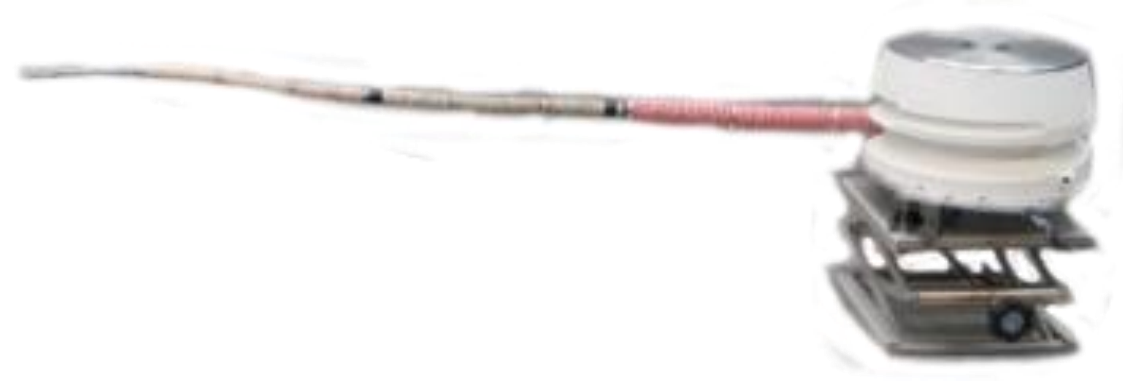

Figure 2.9. Robot with 25 DoF for inspection and reparation tasks in wind turbines [98] 


\subsubsection{Soft robots}

After the study of the continuous robots, traditionally made from rigid materials, new types of flexible structures were considered to allow other types of interactions with the environment. These robots, commonly called soft robots, promised greater adaptability, versatility and security advantages compared to traditional continuous ones.

Other types of actuation such as the pneumatic, not so used up to that time, suffered a great growth alongside this paradigm shift. Some examples of robots that used this types of actuation are the KSI Tentacle Manipulator [139], Air-Octor [99] or the OctArm [100]. Years later, the Festo company also made a relevant industrial manipulator with pneumatic actuation with high performance: the Bionic Handling Assistant $(B H A)[101]$.

First, the Air-Octorfeatured a hybrid performance, provided by a central camera pneumatically pressurized and by external tendons. It consists by 2 pneumatic sections, mainly used to grant consistency and flexibility at the same time. One of the advantages of this design is the hollow design, used to conduct tendons and sensors. These cables are arranged in angles of $120^{\circ}$ and can only exert traction forces. Combination of these 3 cables allow to rotate each section in all the directions. This configuration gives place to 2 DoF, and the soft structure provides a third prismatic one [140]. (Table 2.1).

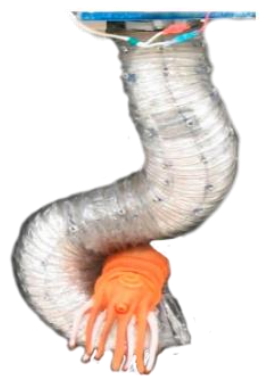

Figure 2.10. Air-Octor, a continuous manipulator robot with multiple sections [140].

On the other hand, the robot OctArm Vis provided by 12 DoF actuated by pneumatic McKibben muscles. It is constructed from a latex covering tube with a double helicoid fabric of plastic mesh. These actuators present a large strength-weight ratio, ideal for soft manipulators.

Furthermore, the Octarm $V$ can grasp objects surrounding them with its own structure. It can move with high precisions and it is able to carry heavy loads. Morphologically, it is divided into three sections, each one provided with 2 axes of rotation and 1 prismatic movement, making a total of 9 DoF [52]. The length of each section was designed to achieve an accumulative bending angle of $360^{\circ}$ in order to manipulate a wide range of objects with different sizes [52].

In addition, it is able of grasping and manipulating objects with a much higher dexterity than Air-Octor. Moreover, it is very flexible, elastic and has good load capacity, but it is difficult to be constructed and controlled. However, Air-Octor has much simpler mechanical design and it is easier to be controlled [141] (Table 2.1). 


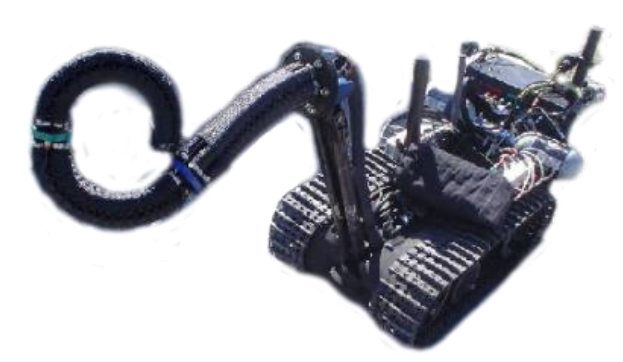

Figure 2.11. Octarm V, a soft robot inspired in an octopus tentacle [141].

As previously mentioned, other relevant soft robot is $B H A$, made by Festo. It is pneumatically actuated and almost entirely made by polyamide, as a result, it is very flexible and lightweight —around $1.8 \mathrm{~kg}$ in weight -. It has 3 main sections, each one with 3 pneumatic actuators, 1 spherical joint and 1 gripper with 3 fingers also actuates pneumatically. The total length of the robot is $75 \mathrm{~cm}$. When the bellow structure receives pressure, it can perform either prismatic or rotational movements. At maximum pressure, the robot can get a total length of $1.2 \mathrm{~m}$. Besides, the robot configuration can be obtained using 12 length sensors. It is capable to stably move so as to carry heavy loads [101]. It is intended for cooperative applications and manipulation tasks because of its inherent safety [142] (Table 2.1).

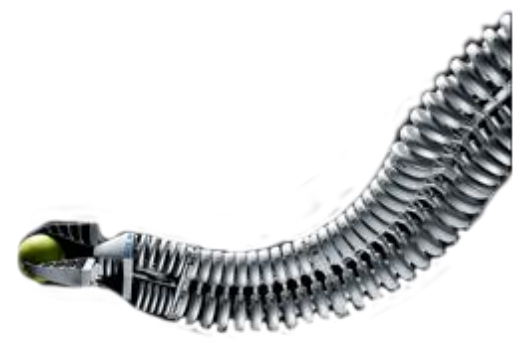

Figure 2.12. Bionic Handling Assistant (BHA), pneumatic soft robot with high dexterity made by Festo [142].

Pneumatic systems have proven to be a solid actuation system for hyper-redundant robots. However, air compressors, needed for such systems, are often bulky, complex, consume lots of energy and have high fabrication and maintenance costs [138].

From 2010, the growth of lighter and easy-to-reproduce soft robots increased. It is possible to find some of these examples in $[20,54,143]$, of which many models were developed inspired by the octopus tentacle [94, 102, 144-146]. In addition, the appearance of these robots has promoted the study and application of novel methods of actuation, using materials with variable stiffness [147], in which are included voice coils [148, 149], electroactive polymers [150], Shape Memory Alloys (SMA) [151], permanent magnets [152], pneumatic sets [153], or even through small controlled explosions [154]. In addition to the octopus tentacle, other biomechanical models have been used based on rajiformes [155], worms [156, 157] and starfishes [48].

Some of the most representative examples of this genre are from the University of Pisa. One of them is a manipulator with a silicone structure actuated by a flexible steel cable to provide prismatic movements, and by a nylon one to provide gripping and flexibility capabilities. Both types of cable are actuated using servomotors. Furthermore, it is capable to adapt its shape around different size and shape objects [102] (Table 2.1). 


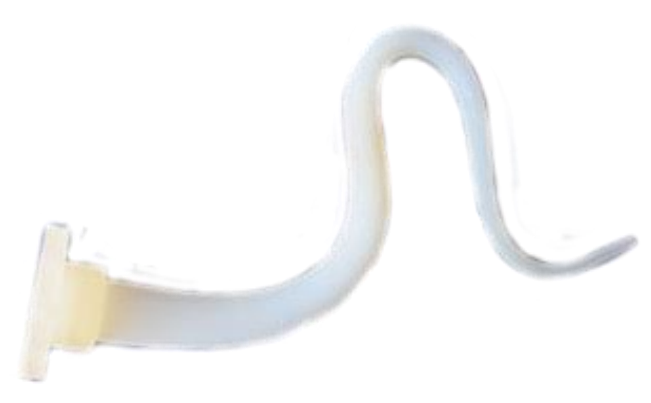

Figure 2.13. Soft robot inspired in a tentacle [102].

Subsequently, over the last years, these mechanical models were improved by implementing a new way of actuation. It is a soft robot composed of a structure of a very flexible braided plastic fibre, actuated by an arrange of $S M A s$, set through both transversal and longitudinal directions to provide the desired deformations [158]. Accordingly, it is a completely soft prototype able to perfectly mimic the movements of an octopus tentacle [94] (Table 2.1).

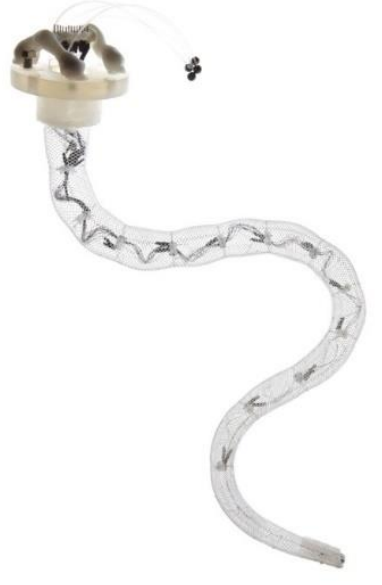

Figure 2.14. Continuous and soft hyper-redundant robot composed by a braided cover and actuated by SMAs [94]. 


\subsection{Modelling}

Modelling continuous or soft robots is a very important step to adequately control them. It consists on the creation of a system able to predict the robot movements by extracting its main properties. Thus, a reliable model is aimed to enable feedback control for robots in which their pose is unknown.

It is worth highlighting that models are also useful even if the robot state is fully known by an ideal sensory system, because methods like Model Predictive Control (MPC) [159], among others, allow the current control actions be optimized taking into account the model-predicted future ones. Nevertheless, traditional sensors do not provide enough information with the required accuracy to reconstruct the whole pose of continuous and soft robots. Furthermore, robot configurations could be obtained from precise motion tracking systems, but models seek for an integrated solution to make the robot independent from external devices.

Models for this type of robots are far from trivial because their non-linearities. It is possible to find classifications of the strategies aimed to solve these challenges in previous works [160]. Based on that, this work proposes three main categories: model-based, learning-based and hybrid approaches (Figure 2.15).

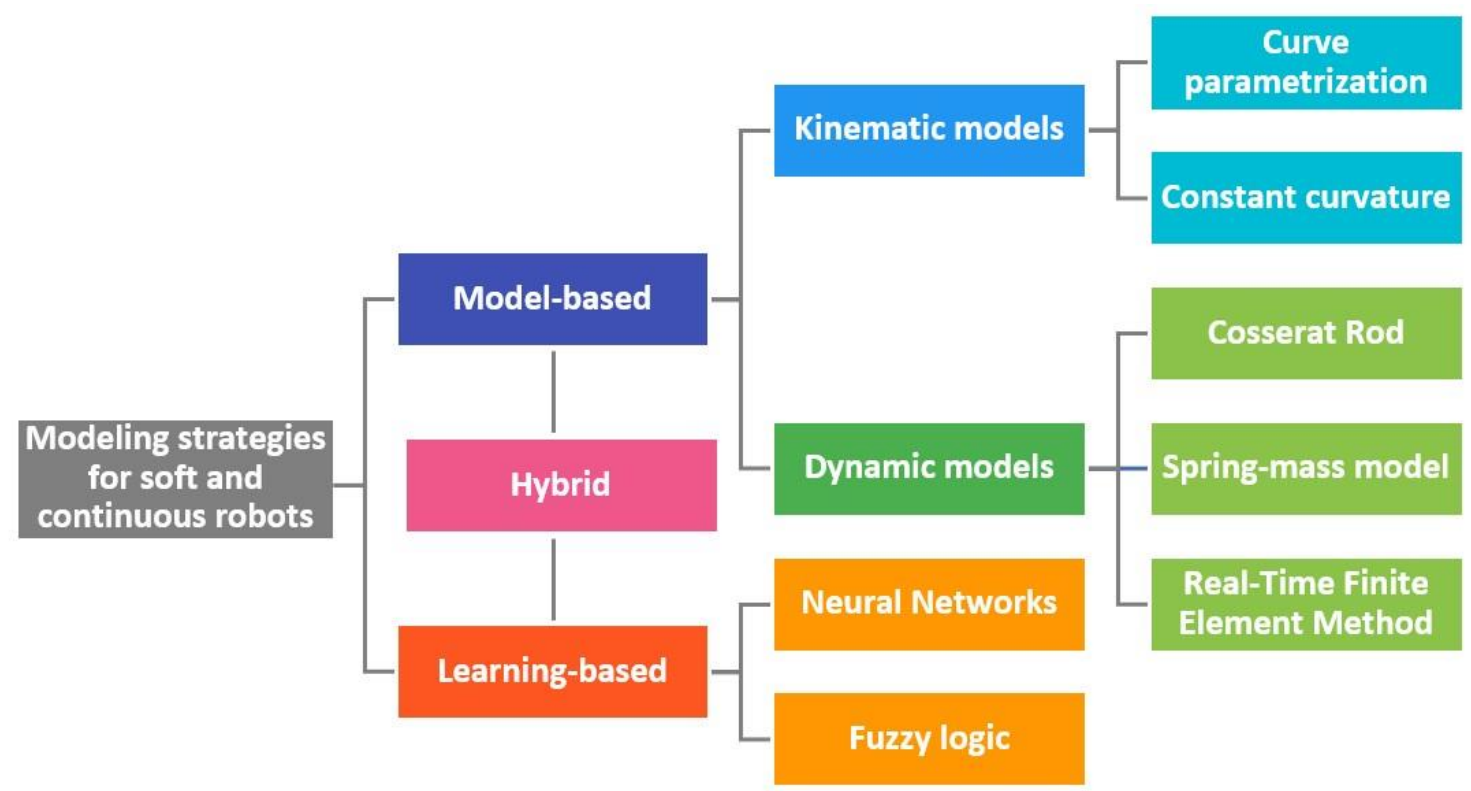

Figure 2.15. Scheme of the main modelling strategies for continuous and soft robots.

\subsubsection{Model-based approaches}

One of the most common strategy consists on creating a user-defined model, which can be either analytical or numerical, but accessible with no need of a training set. These approaches are often denominated as model-based and can be subdivided into 2 main categories: kinematic and dynamic models.

Kinematic models, also called geometric, were the first to be implemented when discrete hyper-redundant robots started to be studied. This is how the curve parametrization models for continuous robots appeared as an approximation of high-redundancy systems (Figure 2.15). One of the first attempts was based on the denominated backbone curves, proposed by [161]. This curve is a piecewise continuous curve (typically the robot centreline) that captures the robot's macroscopic features. This approach was further developed for 
continuous robots using a fitting algorithm. This technique is also applicable to modular hyper-redundant devices, as long as each segment is considered short enough to approximate its shape with the backbone curve. It may be applied for both, inextensible or extensible robots [5, 124]. According to some authors, models that approximate continuum manipulators by discrete hyper-redundant ones could be appropriate $[11,20]$.

Another geometric method, which has been very common in the state of the art because of its simplicity, is the Piecewise Constant Curvature (PCC). In [162], the PCC model foundations are sat down, proposing an alternative method to the backbone curve considering that, in order to use the previously mentioned fitting method, a perfect knowledge of the real robot was required, at least at the kinematic level. Then [126], presented the concept of constant curvature applied in a modified Denavit-Hartenberg framework to determine the kinematics of a hyper-redundant robot. This kinematic model was also proposed to be easily implemented on continuous robots. They demonstrated the viability of this method with the Elephant's trunkmanipulator. The approximation of constant curvature considers the continuous robot body as a finite series of curved elements. But this is possible just ignoring the dynamics of the robot [163]. However, further methods extended the model to include the effects of gravitational potential energy [164].

This approximation is viable when external loading does not significantly affect the robot movements. Otherwise, the constant curvature model can present precision errors up to 50\% [100]. Also, it assumes that the manipulator is uniform in shape, with symmetry on the actuation and minimally altered by torsional effects [165]. Nevertheless, it has been demonstrated that PCC approaches are sufficiently accurate to model a lot of continuum robots with a reasonable error [60]. Related to this method, it was also used a Bernoulli-Euler beam theory to add internal loads for obtaining a more accurate model. In this case, deformations can be parametrized using only three variables: two of them measure the curvature about two axes and one the axial compression [166].

As mentioned, $P C C$ works well when there are no external loads, including self-weight. But this assumption cannot be always neglected [20]. To solve this issue, various techniques based on non-constant curvatures were devised. These methods are called dynamic models, or also mechanical models, because they study the forces involved in the system. One of the most important is the mathematical model named Cosserat Rod (Figure 2.15). This theory understands soft robots as an infinite number of infinitesimal rigid parts. It takes into consideration torsion effects as well as internal and distributed forces and moments on the robot body, which were not taken into account in the PCC model. This approach collects all non-linear effects that affect the curvature. In addition, it is applicable even when the body section is non-constant, in contrast to the PCC $[20,167]$.This kind of model was validated for the OctArm robot, reducing the $50 \%$ precision error obtained with the PCC to a 5\% [168].

Cosserat Rod models have demonstrated to be the finest method to simulate thin structures. It aims to determine the configuration of a rod strand by solving a system of Ordinary Differential Equations (ODE) for one independent variable, rather than a system of partial differential equations that would result from full three-dimensional elasticity [169]. Other example in which Cosserat theory has been successfully applied is in [170]. Moreover, it was recently proposed a discrete approach for this method, in which the continuous Cosserat model is made discrete by assuming a piecewise constant strain along the soft robot. As a consequence, the soft manipulator state is described by a finite set of constant strains [171].

Another mechanical approach is called the spring-mass model. In this strategy, the robot is modelled as a two-dimensional array of point masses and springs. Therefore, all the robot's mass is concentrated in discrete point masses connected by massless idealized muscles, modelled as damped springs [93, 172].

Despite the accuracy of these methods, they are only representative for soft robots with simple geometrical shapes. They are not suitable to capture the movements associated to the morphology. However, there is 
another alternative that accurately represents the possible irregular model's geometry and its non-linear deformations: the real-time Finite Element Method (FEM) [9].

The finite element analysis is based on the generation of a mesh that divides the complex problem into a finite number of small elements. Each element interacts with its adjacent nodes, to which displacements and forces are applied. FEM models tend to the exact solution as the number of elements increases, but this also aggravates the computational cost, hindering real-time applications. So, for this purpose, it is usually recommended to find a balance between computational time and solution accuracy.

Over other approaches for modelling deformations, FEM has the advantage to start from the constitutive law of the material which is experimentally measurable, and through which the elasticity matrix is calculated. This matrix let the position of actuators relate to the end-effector by a complex process, helped by an iterative method based on the model constraints. Also, FEM stands out against previous methods to simulate contacts with its environment, making possible to study soft robotics compliance $[94,173,174]$.

Nowadays, there are new emerging approaches that merge various techniques such as PCC with FEM [175]. Furthermore, it is worth mentioning the SOFA Framework alongside the Soft Robotics Plugin, to be one of the most known applications for FEM purposes in real time. However, new specific software is also proposed in [176], or in [177].

\subsubsection{Learning-based approaches}

Over the recent years, techniques based on machine learning extract the robot's behaviour without previous knowledge of the system and using large datasets (Figure 2.15). Therefore, these methods are also called model-free in the specific literature. Despite this kind of methods are quite recent, they are expected to allow easier modelling and control.

Related to this, one of the main attempts is based on Artificial Neural Networks (ANN), a framework inspired in biological neural networks that learn to perform tasks by considering examples, generally without being programmed with any task-specific rules. In this context, some works use this methodology as a tracking controller that does not require any previous accurate dynamic model of the manipulator [10]. Successively, an adaptive neural network tracking control for manipulators with uncertain kinematics, dynamics and actuator model was developed [71]. Later in 2013, a neural network learned how to solve the inverse kinematics of a soft manipulator moving in a three-dimensional space. After the training, a Feedforward Neural Network (FNN) was used to represent the relation between the manipulator's end-effector position and the displacements applied to the cables [178]. Shortly after, authors validated this method with a real prototype with good results [179].

The learning-based approach allows learning the inverse kinematics problem even in the presence of singularities, without requiring any prior information about the robot, and provided with few open parameters to tune [180]. With this technique, it is also possible to model and control a soft robot using data-driven models that only use mechanical feedback; a very important scheme when sensing is difficult and safe interactions are essential [181].

Other authors have employed membership functions to combine a linearized state-space model, to achieve, overall, a fuzzy modelfor continuous manipulators [72]. This model is then used to design a fuzzy controller supported by a thorough stability analysis.

In general, learning-based approaches are usually preferable for those cases in which the modelling process is substantially unknown to have a high complexity due to environment conditions, such as the influence of gravitational forces, or due to intrinsic high non-linearities. 


\subsubsection{Hybrid models}

Some works like [182] have merged both model-based and learning-based methodologies to solve the kinematics of the compact bionic handling assistant (CBHA). In this case, the robot is first modelled using a quantitative approach for a series of discrete links with flexible joints. Due to the difficulties to solve the inverse kinematics of this kind of robots, a qualitative approach based on neural networks is then used to provide approximated solutions. As a result, the obtained model also works in real time and, to some extent, with an interesting degree of accuracy. 


\subsection{Control}

Regarding the control of hyper-redundant, continuous and soft robots, it is firstly important to remind that they are underdetermined systems, so they can provide a large or infinite number of solutions to satisfy a determined problem. The origin of the challenge is typically related to find one out of many possible solutions and grant the optimal one depending on the application and previous conditions. Additionally, these robots have high kinematic skills and dexterity since have a large number of degrees-of-freedom, so multiple control strategies can be defined depending on the required task.

Usually, three different steps are involved in a control process: first, a low-level control closes the loop for the actuators, then a mid-level, intermediate step links the movement of the actuators to the joint space; finally, the high-level controls manage the whole-body of the robot to perform a specific task such as motion planning movements. Some control strategies are common in this specific field. For example, the inverse kinematics problem aims to find the robot pose that leads to a specific end-effector location [11]. It is possible to find several approaches in the specific literature that try to solve this problem for hyperredundant robots. In recent years, exhaustive methods have been replaced by optimization algorithms because the computational cost of the first ones grows faster versus the number of joints. Thus, the most recent optimization methods studied are Pseudo-Inverse Jacobian [59], Pattern Search [85], Global Search [85], Genetic Algorithms [85], Simulated Annealing [85], Artificial Neural Networks [85] and Particle-Swarm Optimization [78].

However, by solving the inverse kinematics, the pose of the robot is not directly controlled and highlyarticulated robots typically excel when their whole shape is managed instead. That is why the shape-control emerged as one of the most useful for this kind of robots [13]. It is usually linked for teleoperation purposes since a multiple references would be needed to define a specific configuration. Others, such as the followthe-leader strategy, also called nose control, are well known for allowing the introduction of the robot through very constrained spaces inspired by the movement of snakes and other reptiles [12]. On the other hand, motion planning algorithms propose different solutions to move the robot in environments with obstacles [183]. All of these controls are pre-programmed for a given task and environment, implicitly assuming that a reliable model is available.

Hyper-redundant and continuous robots have become popular as tools for inspection tasks since they can work tirelessly in complex or hazardous environments and reach high levels of accuracy and performance. Some of the most propitious scenarios for robot inspection are civil infrastructures, such as bridges, tunnels, roadways, railways, power lines, storage tanks or large facades [184]. These facilities are usually large, complex, constrained or dangerous, so humans cannot inspect them without having adequate resources: e.g., scaffolding, machines, etc. Conversely, robots can cover large areas in reduced times, access to remote and difficult places, and work under harsh conditions and dangerous contexts. Multiple types of robots are used for inspecting civil infrastructures, including not only ground robots [185]or aerial robots [186], but also others capable to crawl and climb [184].

Moreover, sensors and actuators equipped in these robots are essential to close the control loop. They can be very diverse, including those visual, strength-based, ultrasonic, magnetic, electrical, thermal, radio waves or endoscopes [185]. In fact, the use of robotic systems for the inspection of structures implies a set of challenges that need multiple sensors, such as coverage for path planning, data gathering or model reconstruction [187]. Inspection of pipes is another important task in the industry. As an example, a ship usually has among 15,000 and 40,000 pipes with different features (e.g., shape, size and material) depending on their use (e.g., fluid, temperature and pressure) [188]. Therefore, the inspection of pipes is a key task in shipyards that need to be performed not only to build but also for maintenance and repairs [189]. Many scenarios are especially appropriate for robots, but usually require specific morphologies with special 
capabilities. As an example, that is the case of offshore oil and gas industries [190] or other high-risk confined spaces [191].

Hyper-redundant robots can contribute not only to improve the performance in some of the reported scenarios but also to others yet unexplored. Due to their large number of degrees-of-freedom, these robots can operate in constrained environments, reaching unstructured scenarios, navigating in places with a high density of obstacles and operating in narrow spaces like pipes [7]. Additionally, three types of hyperredundant robots that can be used for inspection purposes: manipulators, mobile manipulators and those with locomotion capabilities. Hyper-redundant manipulators are those constrained to fixed bases [21], whereas hyper-redundant mobile manipulators are integrated with automated and mobile bases, increasing their autonomy and workspace [23]. Finally, hyper-redundant robots can also take advantage of their high flexibility to move and manipulate in complex environments like snakes would do [24].

On the other hand, there are numerous reviews that collect different approaches to control soft robots such as [165]. These kind of methods are usually devised ad hoc for each particular robot and application, although there are similarities between robots with similar ways of actuation. Recent works have studied the principles of growth for hyper-redundant and soft robots. Biological systems, through their growing capabilities, are able to deal with uncertainties and damages with a much richer set of possible strategies than state-of-the-art robots [192]. For example, [193] showed that evolved softer robots were found to achieve better performances with simpler growth processes than evolved stiffer ones. Moreover, [194] demonstrated that the learning process can be accelerated when knowledge is transferred in a tadpole-frog as the robot grows, suggesting that growing policies can facilitate learning. Controlling hyper-redundant robots is difficult to achieve, since they rely on a large number of variables. However, when they exhibit in addition growing capabilities, the problem is even more difficult as the state-space is not static, but can increase over time.

The field of growing robots is still in its infancy. They have their origins in hyper-redundant devices introduced in physically constrained environments, like flexible robots designed to navigate into debris [195]. Recent works in growing robotics take their inspiration from vines by using pneumatic actuation to perform steering and elongations achieved by eversion [33]. Those robots are driven by a series of pneumatic artificial muscles that cause approximately constant curvature bending and prismatic movements [196]. This kind of growing robots are not only capable of extending their length but also of contracting it by switching the sign of pressure. An application of this idea is presented in [28], where the system is tele-operated for real-world exploration tasks such as navigation in archaeological sites. The control strategy for such robots is usually aimed to reach a light source, inspired by vines that grow towards the sunlight. Therefore, the growing direction of the robot is aligned to the object of interest. Then, an eyein-hand visual servo controller is adopted to achieve heading alignment or steering by using the images obtained from a camera located at the endpoint [197].

Growing robots based on eversion are very efficient to navigate by locomotion in different environments. Due to their prismatic capabilities, traditional path planning approaches for mobile robots can be suitable for growing robots as well. And, interestingly, soft growing robots can take advantage of intentional obstacle collisions to find passively steer due to their compliance with their surroundings [198]. Other work in growing robots also take inspiration from plants, specifically from roots. In [199] [200], a robotic mechanism for soil penetration was used to build Plantoid, a plant-inspired robot with differential bending capabilities [29]. This kind of robots are able to grow based on additive manufacturing technologies [201]. This approach, compared to eversion, is slower and irreversible, but could give place to more accurate and stiffer solutions. On the other hand, [202] proposes to control the movements of a bio-compatible, tendrillike, soft robot by using a reversible osmotic actuation strategy. This method is based on the electrosorption of ions on flexible porous carbon electrodes. Other controls for growing robots also took their inspiration 
from nature. In [203] has been shown a growing robot that can perform circumnutations, a helical organ movement widespread among plants. These movements could let the robot attach its body to different shapes like climbing plants do, reducing the load of the successive growing sections.

Moreover, different control strategies aimed to grasp objects have been studied before in soft robotics [204]. This kind of actions performed by soft and compliant robots allow objects to be safely manipulated by transferring part of the intelligence to the morphology, often referred as morphological computation [205]. Hyper-redundant robots are also capable of grasping objects by surrounding them with the whole body. For example, in [206], different configurations for a modular robotic system were studied to grasp space debris. However, this behaviour has not been implemented so far for growing devices, which could adapt themselves to grasp bigger and more remote objects. However, growing robots are not continuous and soft by definition. A recent work introduced a sturdy robot that grows like a plant [207]. It has been designed to extend a chain-like appendage flexible enough to twist and turn in any necessary configuration, yet rigid enough to support heavy loads or apply torque to assemble parts in tight spaces. 


\subsection{Teleoperation}

This section presents the up-to-date research in robot teleoperation, specifically in human-robot interfaces and interaction strategies, intended for many types of robots and purposes. Hopefully, this will help to clarify the current research, potential applications and limitations related to this work.

One of the major challenges in robot teleoperation is the difficulty of achieving a precise and reliable representation of the environment. Remote perception and manipulation are affected by factors such as limited field of view (FOV), orientation, camera viewpoint, depth perception, degraded video image, time delay, and motion. Researchers have shown that enhancing the operation feedback plays an important role in decreasing task difficulty and creating a greater sense of operator immersion in a teleoperation environment [208]. Accordingly, some authors have studied the strengths and weaknesses of conventional 2D interfaces against immersive 3D ones. Some of them predict that immersive interfaces can help users to achieve a higher level of understanding of the robot's point of view and situational awareness [209].

Several works related to mobile robotic applications, agree at selecting virtual and augmented reality interfaces in order to improve performance [210-213]. One example of enhancing situational awareness is Flying Frustum, an AR interface aimed to control semi-autonomous Unmanned Aerial Vehicles (UAV) using a 3D interactive printout of the terrain. In this work, UAV operators could use pen-based interactions to input flight paths and commands to the UAVs by sketching directly on the physical topographical model of the terrain [214]. Moreover, many works claim that VR technologies applied to robotics can reduce the cost of expensive specialized systems in manufacturing [215-217].

Concerning the teleoperation of service robots, immersive realities can be used for surveillance and remote interventions in our homes. Conducted experiments with two MR visualization modalities were compared with standard video-centric and map-centric perspectives [218]. As a result, novice operators experienced a higher workload and were less efficient than expert ones. And about the interfaces, the performance was significantly inconclusive. According to the authors, this is due to a high complexity of different factors such as the operation environment, the mission's objectives, the robots' capabilities or the choice of performance metrics. In another work, teleoperation enabled operators to establish effective communications between humanoid robots and people in order to avoid the automatic recognition difficulties of spoken language [219].

In the hyper-redundant robotics scope, teleoperation schemes often follow traditional master-slave architectures. This is reasonable since these robots with a large number of DoF are usually perceived to need a replica with the same number of DoF in order to exploit all their kinematic skills. However, using masters and slaves with different number of DoF is a common practice. For example, a Phantom Omni haptic device (master) was used to accurately teleoperate the end-effector of an articulated snake with 12 DoF (slave) for minimally invasive surgical procedures. In these situations where the number of DoF of the master is lower than in the slave, it is necessary to obtain a mapping relation between each other [220]. Another example uses the same master to teleoperate a tendon-driven continuous robot by using a VR interface in real-time, also intended for surgical procedures. This research affirms that the kinematic structure of the master is very dissimilar to the kinematic model of the continuum robot, so the teleoperation strategy may not be the best [221]. Such dissimilarities were also studied trying to teleoperate a redundant continuous manipulator using a non-redundant rigid-link master. These differences were minimized using feedback linearizing task-space controllers [222]. In all these works, the inverse kinematics is interrelated between the master and slave for the end-effector only. Then, only the inverse kinematics problem is solved but whole-body controls are not managed. This work will study such wholebody movements to be the ones that fully take advantage of the kinematic capabilities of these robots for remote operations. 
Different interaction tools are used to teleoperate robots, varying from traditional and mechanic peripherals to multimodal ones. On one hand, physical tools vary from game controllers, electronic devices, keyboards, mouse devices, joysticks or Phantom haptic devices, among others. Depending on the field of application and the robot kinematics, one can be chosen against another. For example, to fly a UAV, specific physical controllers with buttons and axis are commonly used [209].

Human gestures without physical intermediaries are another way of controlling robots in teleoperation. They can be advantageous because are easy to use, robust, fast and can be used in a wide range within the field of view [223]. Such interfaces are oriented to tasks that can be performed hands-free. In fact, some authors claim a higher prominence of devices like the Leap Motion sensor for the future of human-machine interaction applications [224]. Some works describe vision system interfaces that track human gestures for relatively simple robot commands such as up-down, stop, turn, approach and go [225]. Others, developed a robotic wheelchair controlled using face movements [226]. Moreover, it is worth highlighting that these strategies are also used to control UAV by using body postures [227]. Usually, vocabulary rules or ad-hoc methods are used to select hand gestures. Some authors affirm that analytical approaches are better to design gesture vocabularies for multiple objectives for psycho-physiological and gesture recognition factors [228]. Accordingly, gesture-based techniques can be a valid alternative to physical master-slave architectures since they can be cheaper and more intuitive [229].

Voice commands are also useful especially in environments where manual control is difficult or when both hands of the operator are busy, as in the case of ROBTET, a robotic system for maintaining live power lines. In such a system, users use voice commands while both hands perform other control tasks [230]. Other projects compared the utility of manual and speech inputs for several UAV control tasks. Results showed that using speech was significantly better than manual inputs in terms of task completion time, accuracy, flight and navigation measures and pilot ratings [231]. Voice commands have been used in telerobotic surgical systems as well, as in ARTEMIS, an Advanced Robotics and Telemanipulator System for Minimally Invasive Surgery. The main components are two master-slave units guiding the surgical instruments and a remotely controlled endoscope guiding system. This last one can be guided by a simple joystick, voice control or automatic camera tracking [232]. In addition, voice can be a powerful tool to use in teleoperation of humanoid robots for being convenient and simple, with less workload and learning time involved [233, 234].

Natural language interfaces can be applied to make the use of intelligent robots more flexible, such as in the case of the autonomous mobile two-arm robot KAMRO [235]. Combination of gesture and voice strategies give place to multimodal interfaces, providing a large range of interactions, intuitive to humans and advantageous to service robots [236]. There is evidence that multimodal displays and input controls have great potential towards improving the teleoperation performance [208]. In fact, some authors claim that these approaches increase awareness of surroundings, cue visual attention, and convey a variety of complex information, especially when the visual channel is heavily loaded [237]. 


\subsection{Mach, a discrete hyper-redundant robot}

The robot presented in this section was designed by Ivan Rodríguez, student of the Centre of Automation and Robotics (CAR, CSIC-UPM). However, it will be explained because it will be further used for control and teleoperation purposes. It is intended to perform inspection tasks in constrained environments. For that purpose, the design should take into account the following requirements. First, it needs to be hyperredundant and have a large number of degrees-of-freedom in order to increase its dexterity in unstructured scenarios. According to the size and potential applications, a diameter from 100 to $120 \mathrm{~mm}$ and an extended length in between 700 and $900 \mathrm{~mm}$ would be preferable. Additionally, the robot should be modular to allow different lengths and the actuation box should be reusable for different redundant manipulators. At the same time, it needs to be long lasting, so it should be made by resistant materials. Moreover, the robot should be able to carry payloads of $1 \mathrm{~kg}$ to move different sensors for inspection purposes. And lastly, it should have high accuracy, repeatability and resolution.

As a result, the robot is designed to be hyper-redundant and discrete. As it will be explained, it has 14 degrees-of-freedom and 7 distinguishable and rigid sections. Its long structure will be driven by tendons and designed to carry out different applications, inspection tasks included. Following sections will describe useful information of the mechanical design, actuation system and electronics of the robot.

The design of the robot as a mechanism is constrained by the previously mentioned requirements. Under these circumstances, it is very important to highlight that a good design is the result of a compromise between different requirements that may conflict with each other.

The robot was required to be modular, which usually consist on the repetition of a module in the kinematic chain. Sometimes this module is designed to decrease in size from the base to the tip. However, the robot is less modular and expandable following that scheme. Therefore, the size of the modules was kept constant along the kinematic chain with a diameter of $108 \mathrm{~mm}$. In every module, the links were extended by appending a rigid section to the joints to increase the total length, which is $L=675 \mathrm{~mm}$. And a set of universal joints were selected to fit the desired size requirements.

A total of 7 modules were selected as adequate to obtain maximum bending angles of 33을 between consecutive sections and an accumulated total bending angle of $230^{\circ}$ in the whole body. This parameter is very important in hyper-redundant robots since it is useful to quantify their dexterity. Additionally, the material for the base module needed to be light and resistant, so aluminium was chosen as a good candidate. As a result, every module weighs $m=0.681 \mathrm{~kg}$ and requires an appropriate actuation system (Figure 3.1). 


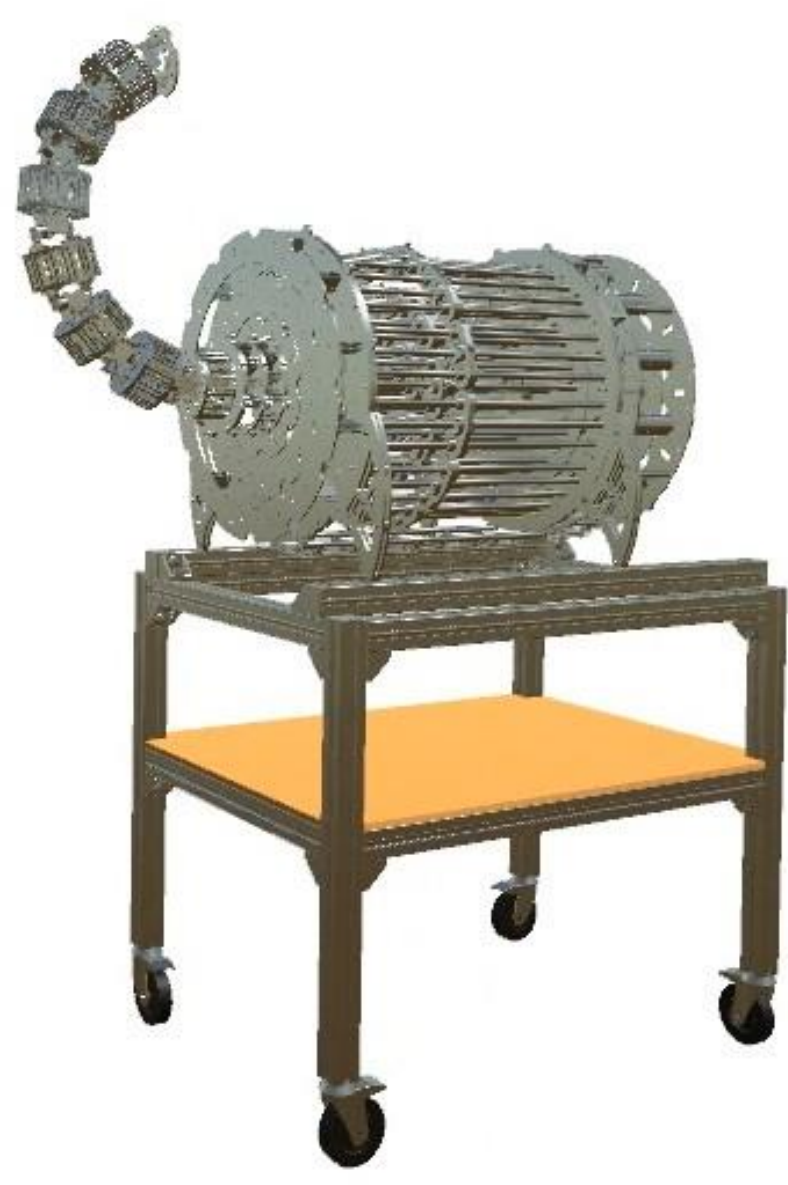

Figure 3.1. Complete mechanical design of a tendon-driven, discrete and hyper-redundant manipulator with 14 degrees-of-freedom and 7 sections.

As a remainder, and depending on the location of the actuators, it is possible to distinguish between distributed actuation, when it is located within the robot mechanism itself, and remote actuation, in those cases where the movement is transferred from an external location.

Robots devised for inspection tasks can be very different in size depending on each application. Distributed actuation systems are not possible to be reused for robots with different sizes. Additionally, that setup would highly increase the total mass of the robot and, consequently, the power and the size of the actuators as well. Thus, the actuation system was chosen to be remote from an external actuation box based on DC motors and twisted steel cables.

Based on this type of actuation, it is possible to calculate the nominal torque needed for the presented robot. Static forces were only taken into account since dynamic ones can be neglected when the robot performs with high cycle times. Figure 3.2 depicts a free body diagram of the robot body with a straight configuration, perpendicular to the action of gravity. Theoretically, this configuration is the most disadvantageous pose for the robot in terms of load. The torque $\left(M_{1}\right)$ will find its maximum value in the first joint $\left(q_{1}\right)$, as a result of the influence of gravity $(g)$ in the mass of every module $(m)$ multiplied by their distance to the first joint $\left(I_{i}\right)$. Also, a payload of mass $m_{p}$ was included according to the robot requirements, located in the endpoint at a distance of $l_{p}$ from the first joint (Eq. 3.1). 


$$
M_{1}=\sum_{i=1}^{7}\left(m \cdot g \cdot l_{i}\right)+\left(m_{p} \cdot g \cdot l_{p}\right)=11.92+6.88=18.8 \mathrm{~N} \cdot \mathrm{m}
$$

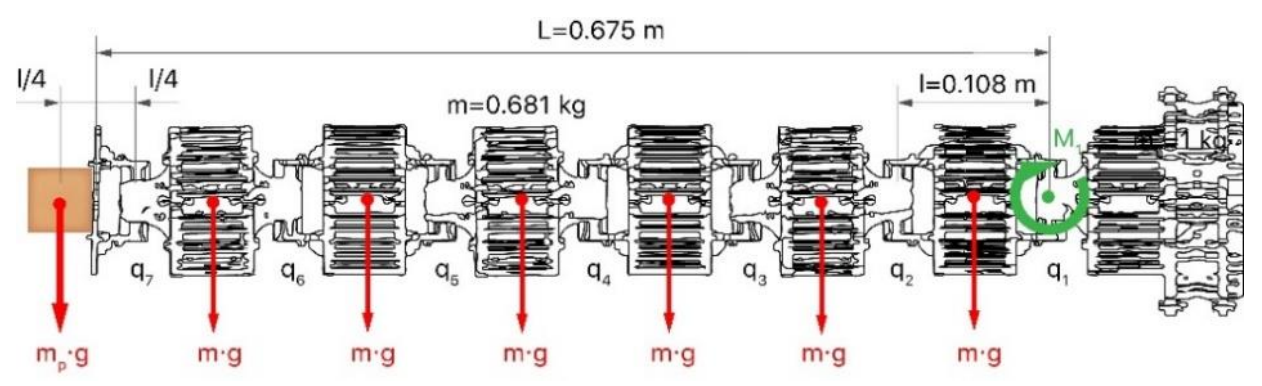

Figure 3.2. Free bodydiagram of the required torque exerted by the firstjoint to balance a payloadand the weightof the robotbody.

Therefore, the selected DC motors are required to have enough power to balance the torque generated by the weight of the robot and the payload. A factor of safety is applied in numerous steps of the design, this one included. Also, it is important to select the motors depending on their speed. In this case, the torque generated by each motor is transferred to a threaded spindle with a pitch of $0.4 \mathrm{~mm}$ that converts the rotation to a linear movement. Then, a carriage with internal threading and bearings is screwed in the spindle and fixed to 2 smooth rods to obtain the linear translation. In fact, this system is a mechanical speed reducer that will determine the resolution, accuracy and speed of the robot. Following this design, the spindles and rods needed to have a minimum length equal to the maximum displacement of the tendons.

The resulting mechanical design is very sturdy and durable. It has been designed to have a high resolution, accuracy and moderately high speeds for inspection purposes (Figure 3.3).

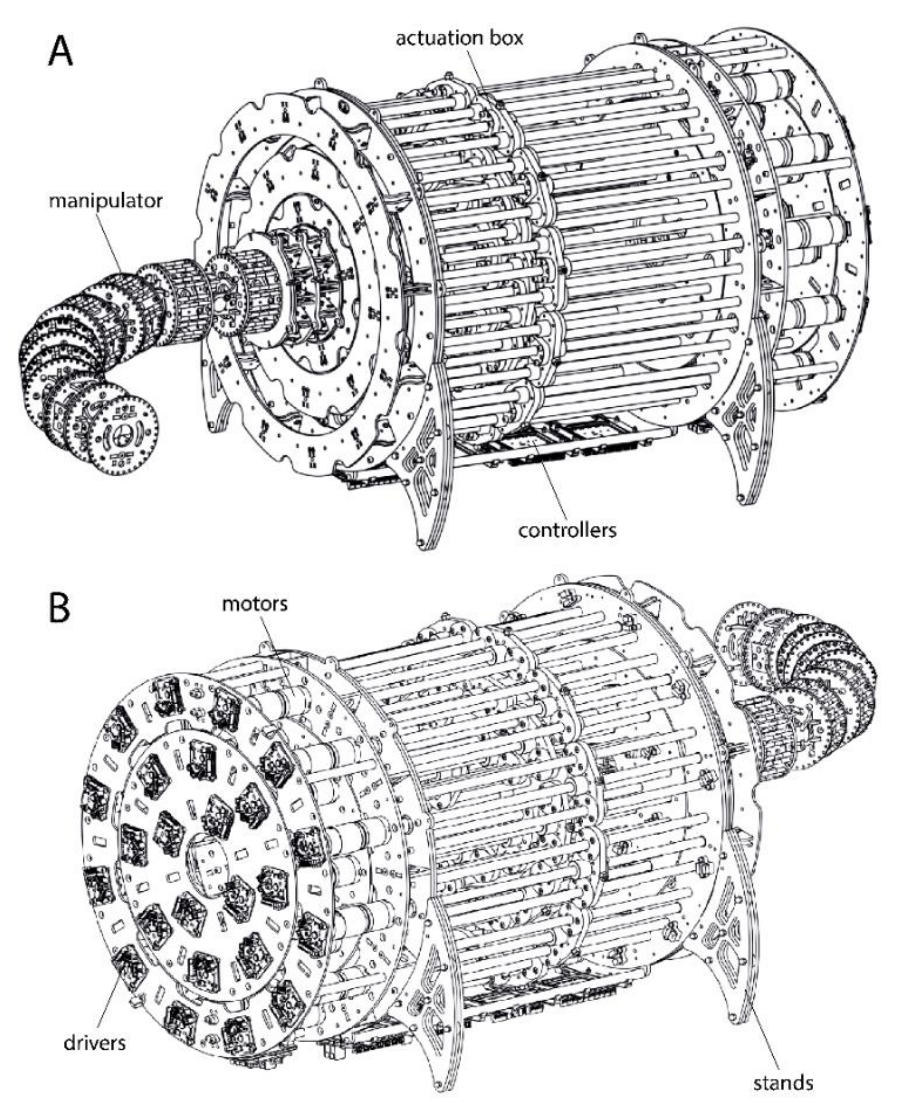

Figure 3.3. Perspective (a) front and (b) back view of the complete design of the hyper-redundant robot. 
The lack of commercial solutions with high standards of synchronization and communication capacity leads to the customization of the electronic system for this particular robot. For instance, an appropriate synchronization of the actuators in tendon-driven manipulators is crucial since they need to move in coordination.

As a result, each driver of the robot is designed to obtain a closed-loop system using the feedback from an encoder (Figure 3.4a). All of them are devised to allow communications via Controller Area Network (CAN) bus to simplify the wiring process. Then, other 4 additional circuits are manufactured: board to communicate the state of the limit switches (Figure 3.4b), central controller to manage all the communications (Figure 3.4c), 24V power board (Figure 3.4d) and a 9V power board (Figure 3.4e).

A

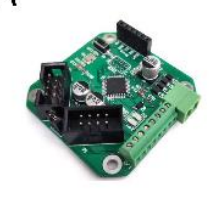

B

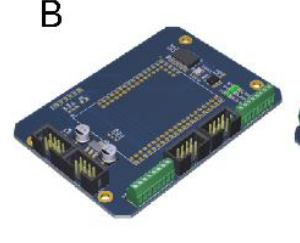

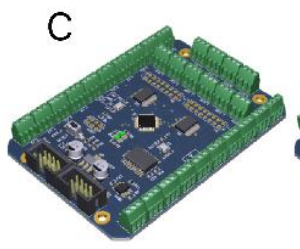

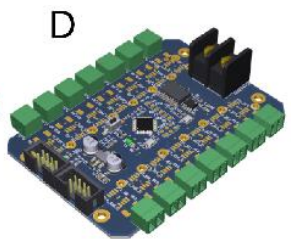

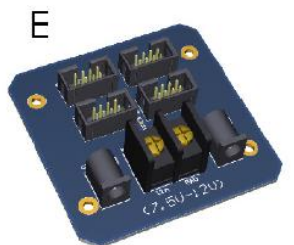

Figure 3.4. Customized design of different electronic boards. (a) Motor drivers for a closed-loop control using encoders. (b) Electronic board to supervise the limit switches. (c) Central controller to manage all the communications. (d) $24 \mathrm{~V}$ power board to supply the motors. (e) 9 V power board to supply energy to the rest of the electronic boards. 


\subsection{Magno, a continuous magnetic manipulator}

This continuous robot was inspired from the Continuum Robots Laboratory (CRL) from University of Toronto Mississauga but fully developed in the Centre for Automation and Robotics (CAR, CSIC-UPM). It is a device that uses magnetic modules, a flexible core and an actuation system to obtain bending and prismatic movements.

First, the flexible core functionality aims to be useful as a backbone of the robotic device and manage bending movements. Its properties will characterize the whole robot movements the most since it constitutes the central part.

By alternating the orientation of the modules that generate magnetic fields, it is possible to create repulsion between each base module with their adjacent ones, creating a damping force when they interact with each other that resembles of an invisible spring. Such magnetic damping mechanism, provided with a central hole, can move along the flexible core providing prismatic movements in each section. In addition, these magnetic modules constitute as part of the robot body and avoid the use of additional elastic elements such as springs. Thus, the possibility of miniaturizing the size of this type of robots increases considerably, which is desirable for many applications such as the case of surgical interventions (Figure 3.5).

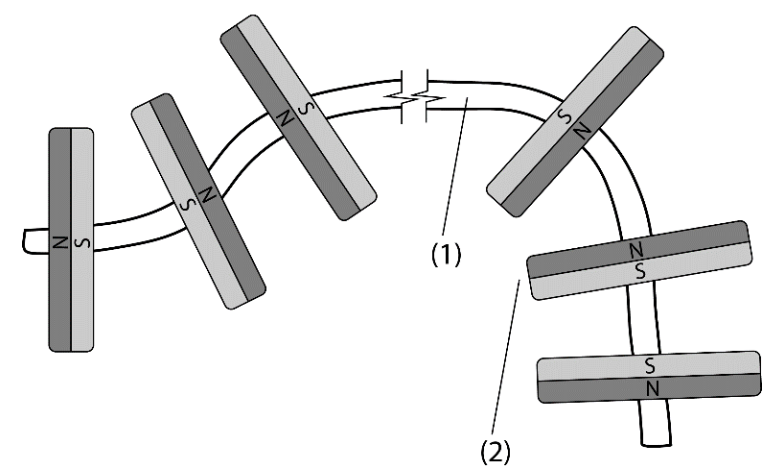

Figure 3.5. The magnetic modules (2) are alternated facing opposite poles throughout the flexible core (1), allowing prismatic movements like a spring would do.

As can be seen, the modules with magnetic fields are in alternate orientations so there is a repulsive force between modules so a damping force is obtained. In the case that magnetic fields are created from permanent magnets, they must alternate their orientation so that two south-south or north-north poles face each other to achieve the repulsion effect. However, the magnetic fields can also be formed by electromagnets, such that the force between them can be variable, providing additional degrees of freedom.

In addition to the modules with magnetic sources, an actuation system will be responsible for controlling the aforementioned prismatic movements, as well as those derived from the bending of the central core (Figure 3.6). The actuation system will exercise its function on the modules and can be of any type, whether by transmission of motors and cables, shape memory alloys, artificial muscles, hydraulic or pneumatic micro actuators, etc.

The number of modules with magnetic sources and the number of effective actuators per module can be variable and dependent on the desired number of degrees of freedom, with a minimum of 3 per section. Not all the modules from the structure should be directly actuated but used as intermediates to compose a section. Thus, the design is modular so it can be reconfigured as desired. The movement of this robot is nonlinear. 


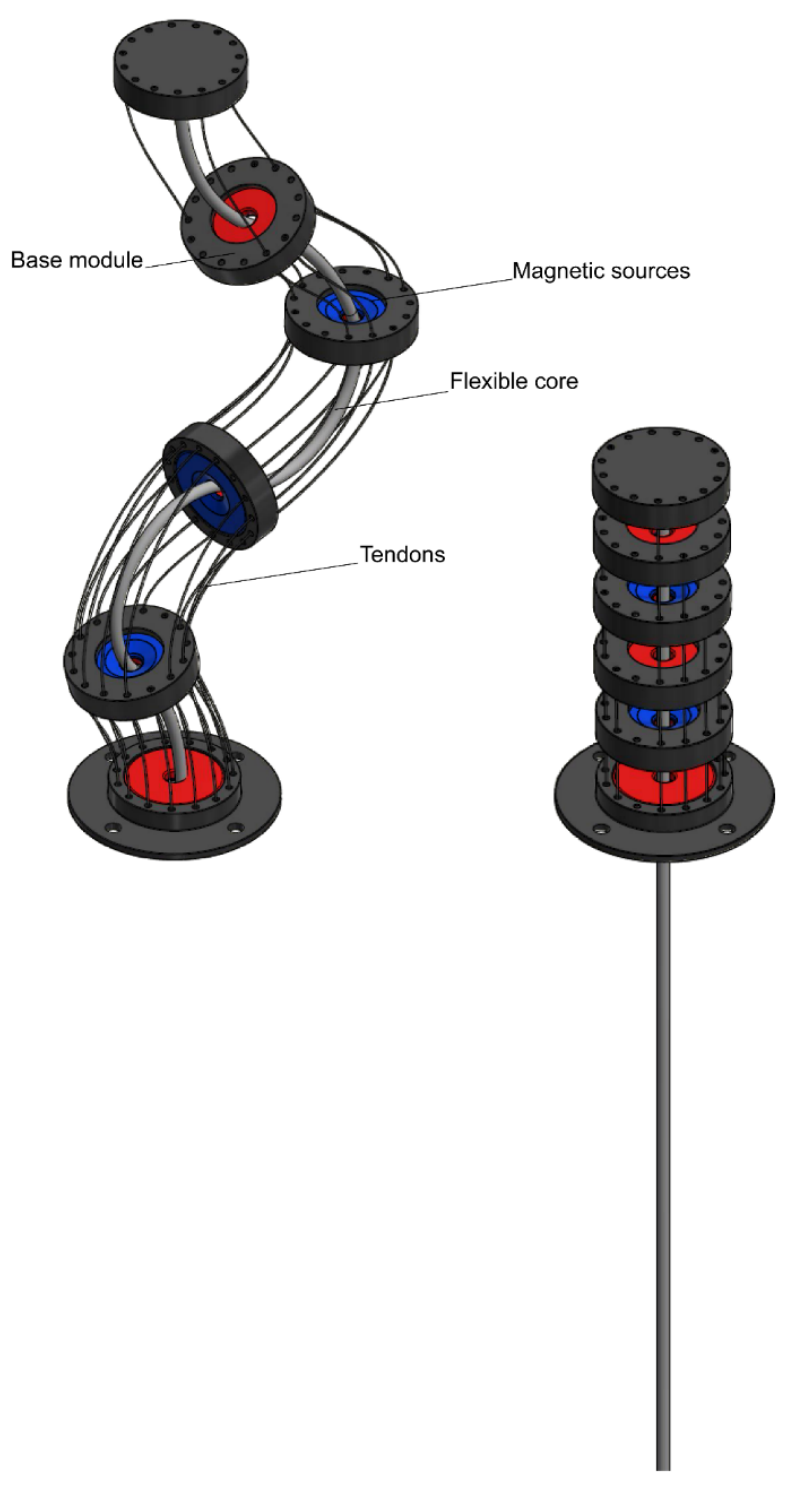

(a)

(b)

Figure 3.6. Full mechanical model of Magno, a continuous manipulator with bending and prismatic capabilities. (a) By actuating each cable independently or by pairs, it is possible to control bending. (b) If the three cables of each section are actuated simultaneously, then it is possible to control prismatic movements. 


\subsection{Kyma, a tendon-driven soft robot}

Kyma is a continuous soft manipulator personally designed in the Center for Automation and Robotics, CARCSIC-UPM (Figure 3.7).

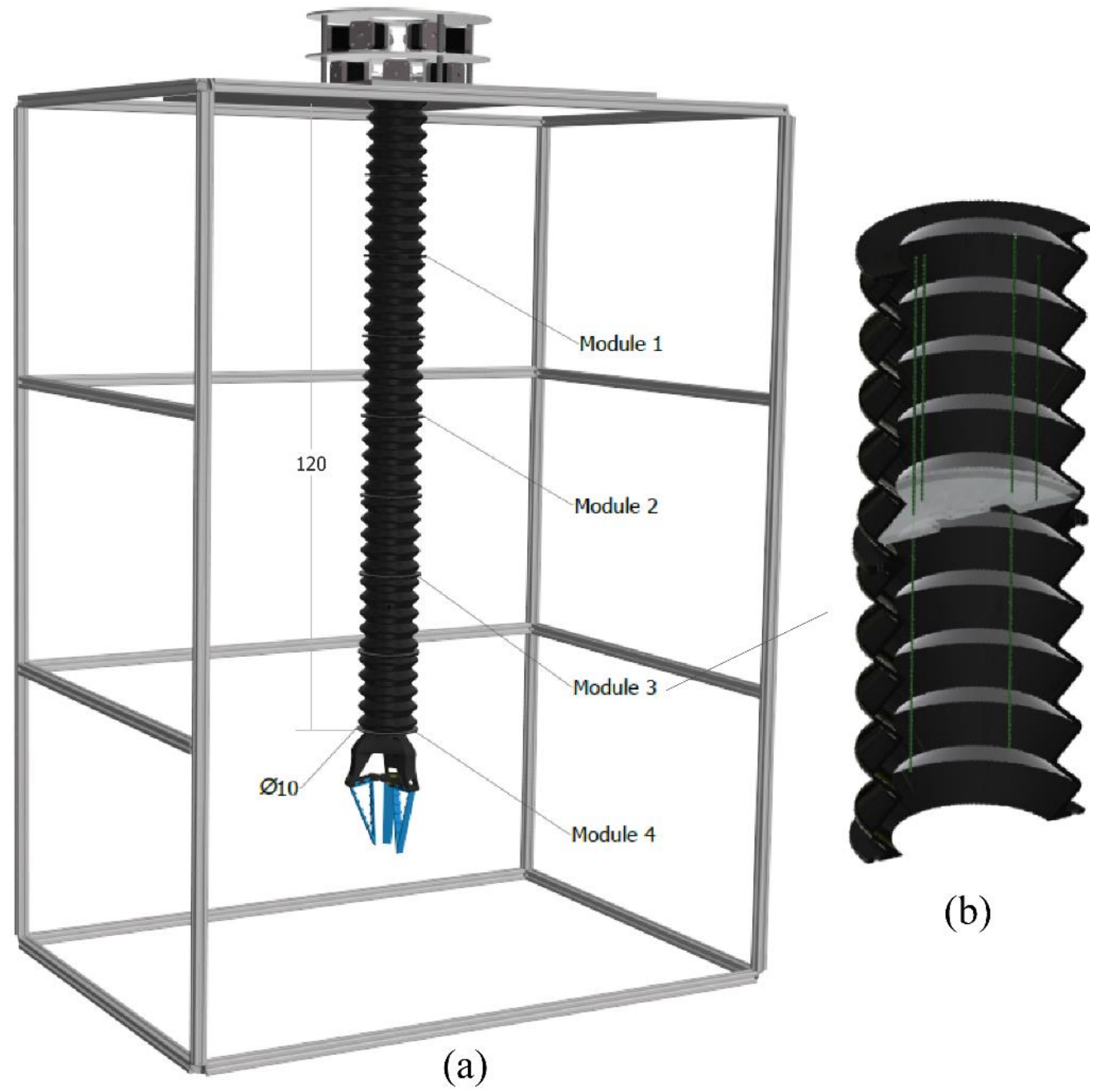

Figure 3.7. (a) Mechanical model of the soft manipulator Kyma, composed by 4 equally spaced modules actuated by tendons. (b) Detail section of one module. Dimensions in centimetres.

Its structure is based on a set of wavy bellows that allow both bending and prismatic movements. In fact, it has high telescopic capabilities since it can shorten up to a $25 \%$ of its full length of 1.2 meters. This is granted by its soft body composed by flexible Polyvinyl Chloride (PVC) bellows of $100 \mathrm{~mm}$ in external diameter and $2 \mathrm{~mm}$ in thickness. Thus, when a single actuator makes a displacement, it usually combines both bending and prismatic movements, giving place to a high non-linear model (Figure 3.8). 


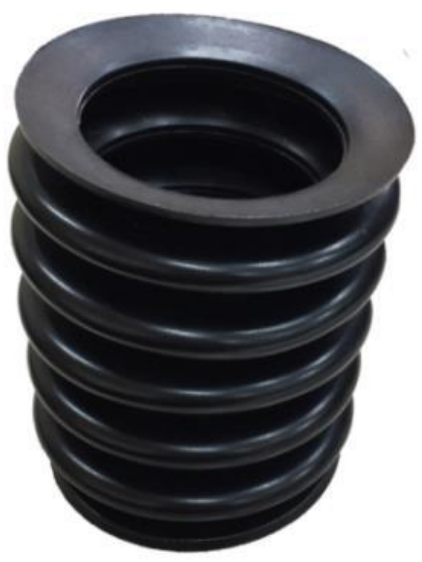

Figure 3.8. Soft PVC bellow used to construct the main structure. The morphology allows both bending and prismatic capabilities.

Its body is divided into 4 sections, each one of them actuated by 3 angularly equidistant tendons at 120 , made by Ultra-High Molecular Weight Polyethylene (UHMwPE), a very strong and light fibre. Other geometrical combinations were also tested but showed less stability and lower torques (Figure 3.9).
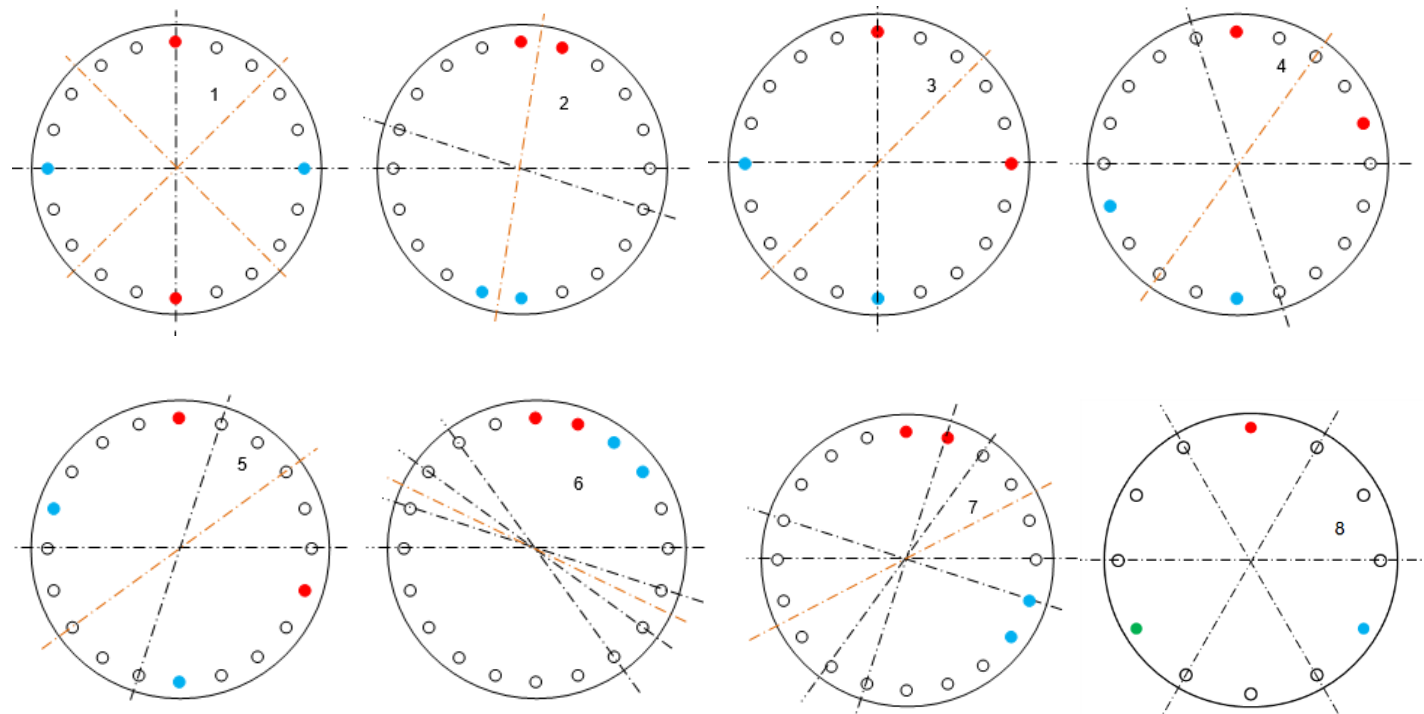

Figure 3.9. Different configurations to distribute the cables in a single module. Same colour dots represent the movement of a motor and a cable in two directions, black axis represent independent actuation rotations and orange ones the combination of all of them.

These cables are anchored in 4 fiber-reinforced plastic discs, encapsulated inside the bellows (Figure 3.10).

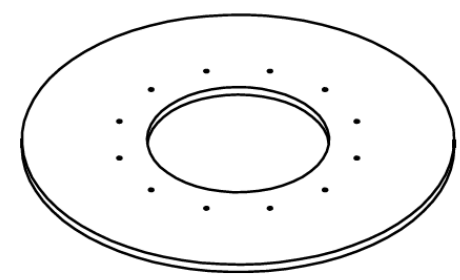

Figure 3.10. Inter-modular disk made from fiber-reinforced plastic.

Kyma relies on a total of 12 stepper motors in the external actuation box, capable to pull the 12 tendons in a controlled manner (Figure 3.11). 


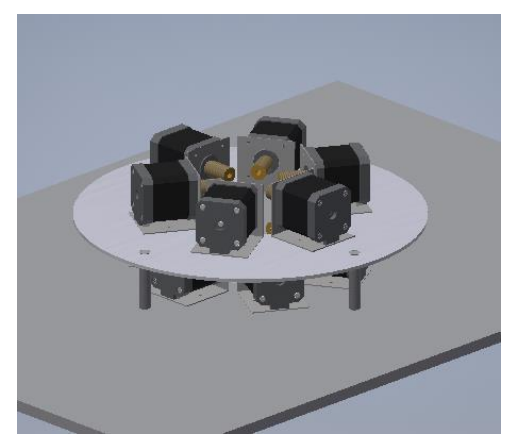

Figure 3.11. Actuation box with 12 stepper motors located in two parallel planes.

In this way, it is possible to obtain 2 rotational degrees-of-freedom combining lengths one by one or in pairs, and 1 prismatic degree-of-freedom when the three of them act simultaneously for each section. Thus, the whole robot has 12 controlled degrees-of-freedom: 8 rotational and 4 prismatic. Because of this, the discs have 12, 9, 6 and 3 holes from the base to the tip respectively, and distributed in circumference, to make way or to anchor the cables.

The length of the 12 tendons is independently controlled by using the motors, all of them synchronized using a hierarchical control structure composed of 1-master and 3-slave processors. Also, the robot is equipped with an end-effector gripper that allows carrying out pick and place operations. However, it will not be considered for the next modelling process since the object of study is just the soft body.

Additionally, the robot has a handcrafted, on-board sensor system to capture its pose in real time. It is constructed based on an array of Inertial Measurement Units (IMU) and further information can be found in Section 4.2 . 


\subsection{Other design efforts}

\subsubsection{A magnetorheological approach to soft robots}

In addition to the previous approach that used solid, permanent magnets to provide a damped mechanism, other areas related to magnetic actuation have been explored during this thesis. The result of this research have led to magnetorheological soft robots. This kind of robots were constructed from soft materials such as silicone but, during the curing process, both ferromagnetic and magnetic small particles were added to the mixture.

The interesting part is that this magnetic blood is susceptible of being actuated by another magnetic source, which can be either permanent or variable, in the case of electromagnets. This is very interesting because, by using this strategy, the structure of the robot is acting as both a structure and an actuation mean, allowing incredibly miniaturization efforts.

Furthermore, as another disruptive contribution, these robots can be remotely actuated without a mechanical or pneumatic transmission but just with forces involved, even across magnetically permeable materials. These kind of robots have been previously introduced in the state-of-the-art $[238,239]$ but in this document a novel conceptual idea will be proposed as Figure 3.12 depicts.

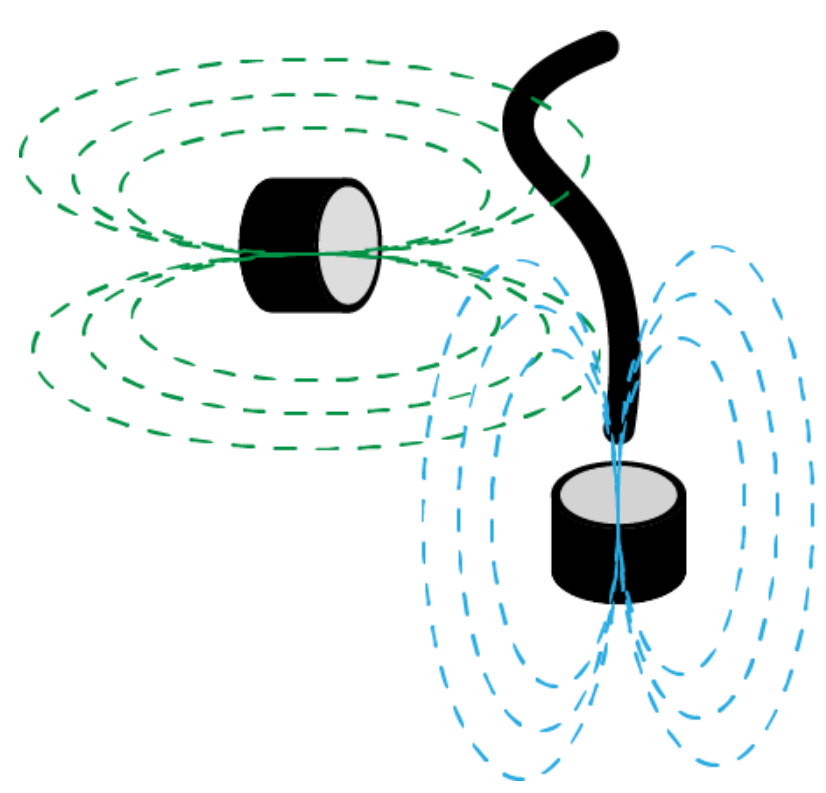

Figure 3.12. Three-dimensional magnetic field created by the conjunction of multiple magnetic variable sources to control the movements of a magnetorheological, susceptible robotic device.

Usually, these robots are created by silicone and iron particles so they behave like a ferrofluid; always attracted by magnetic sources but not repelled. However, it would be possible to create one of this robots with magnetic particles efficiently aligned to obtain two poles or a sequence of coupled dipoles while still retaining the soft structure.

This idea relies on the construction of a magnetic field in the 3D space with different variable magnetic field forces in multiple directions so a susceptible device can be controlled within. The magnetic forces could be created either with rotating permanent magnets, typically stronger and more compact, or with 
electromagnets. Moreover, the robot inside could adopt different shapes by contactless actuation or even levitate. Smart design ideas in the robot morphology could even facilitate the movement along certain directions.

This prototype was constructed and some valuable conclusions could be derived from it. Firstly, the silicone material and the quantity of solute should be optimized to minimize the damping between the magnetic sources and the susceptible particles. In addition, a real-time Finite Element Method or similar modelling technique would be needed to control the forces in the space and, consequently, the shape of the robot. This kind of software tools do not currently exist. Additionally, the applications of this technology seems to be quite limited for now but certainly has a lot of potential for research purposes.

\subsubsection{Synthesis of hyper-redundant robots using the Fourier transform}

The mechanism synthesis determines the size and configuration of mechanisms that shape the flow of power through a mechanical system to achieve a desired performance. In this context, previous works have already proposed some efforts to synthesize serial manipulator robots using the Fourier Transform [240].

The Fourier transform is a mathematical tool that allows you to convert signals from the domain of time (or space) to the frequency domain. Typically, we are used to seeing the Fourier transform applied to onedimensional signals in temporal domain.

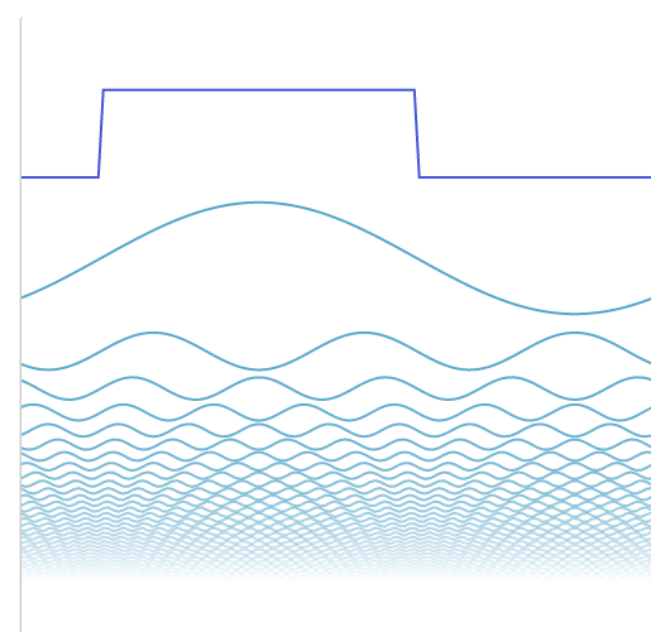

Figure 3.13. Composition of a squared signal from the sum of multiple sinusoidal signals obtained from the Fourier transform

However, it is possible to apply the Fourier transform also for two-dimensional spatial signals by using complex numbers.

Fourier Series: $u(t)=\frac{a_{0}}{2}+\sum_{n=1}^{\infty}\left(a_{n} \cos 2 \pi n F t+b_{n} \sin 2 \pi n F t\right)$
Substitute: $\cos \theta=\frac{1}{2} e^{i \theta}+\frac{1}{2} e^{-i \theta} \quad$ and $\quad \sin \theta=-\frac{1}{2} i e^{i \theta}+\frac{1}{2} i e^{-i \theta}$

Complex Fourier Series: $u(t)=\sum_{n=-\infty}^{\infty} U_{n} e^{i 2 \pi n F t}$

If the $\sin$ and $\cos$ functions are substituted by an exponential function, it is possible to express the same equation with complex numbers. Actually, this exponential function can be expressed as a two-dimensional curve usually called epicycle. One epicycle can be understood as the curve obtained from the rotation of a set of sequentially joined segments. 


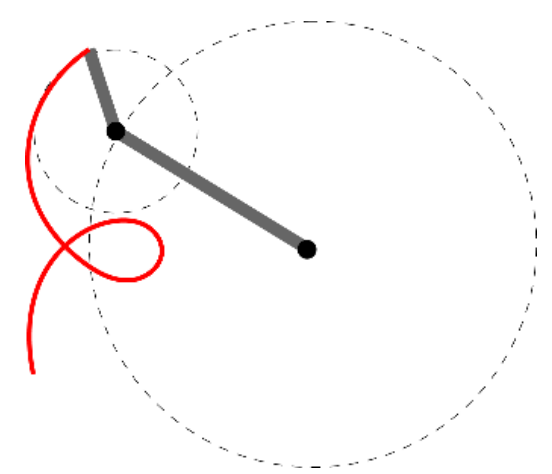

Figure 3.14. Epicycle resulted from of the rotation of two segments, represented in red.

In other words, by using the Fourier Transform, it is possible to find a set of segments that, constantly rotating in different directions, result in a previously defined trajectory. Thus, the Fourier transform provides knowledge about the length of those segments, their initial phase and the frequency of the rotation.

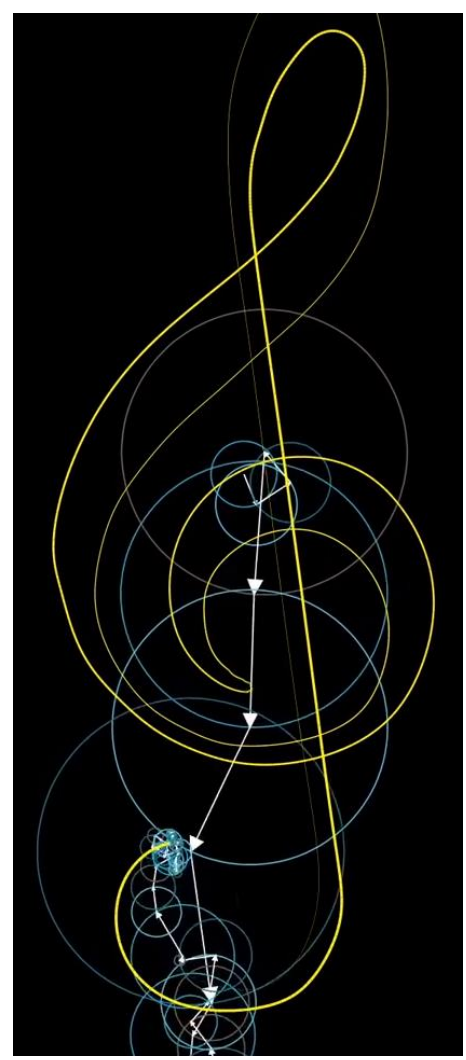

Figure 3.15. A set of multiple segments that rotate can result in a complex trajectory under the initial given conditions.

Epicycles could remind to the trajectories obtained from complex pendulum systems with zero gravity conditions. Just the double pendulum is already one of the most simple, chaotic systems. The trajectories obtained from the tip are highly dependent on the initial conditions when the dynamic actions are not neglected. 


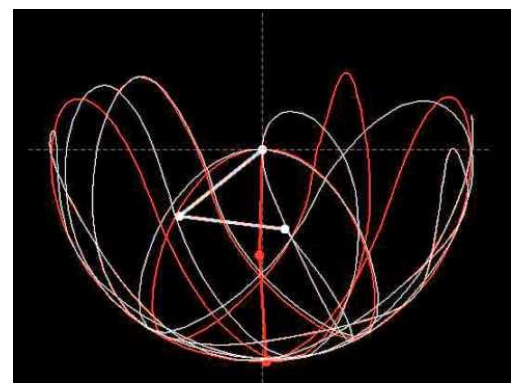

Figure 3.16. The trajectory of the tip of a double pendulum is a chaotic system.

However, by using the Fourier transform it is possible to control the movements of such systems neglecting the dynamic actions and just studying the kinematic actions.

Each segment that give place to an epicycle could be understood as links from a robot that rotate to result in a given trajectory. Consequently, it is possible to control the movement of a robot, and specifically those hyper-redundant. The higher the number of links, the higher the redundancy of the robot would be, and the higher also the precision to perform a given trajectory. Previous past efforts have acknowledged this problem for regular manipulators but have not been well-adapted to the needs of hyper-redundant ones.

There are some key insight conclusions derived from several programmed simulations about this for hyperredundant mechanisms:

- The design of a hyper-redundant robot can also constitute as an integral part of their control. Thus, the Fourier transform can be a design criterion to choose the level of redundancy and the morphology of a purely kinematic system.

- The epicycles obtained using the Fourier Transform give place to a desired trajectory as a result of a cumulative sum at the end-effector. Since the sum is mathematically commutative, it is possible to alter the order of the links rotating to give place to a given trajectory. This property grants a huge flexibility in order to design hyper-redundant robots since there are many schemes that can be proposed from sorting the links by length, direction of rotation, speed or initial phase.

- The higher the number of links or modules of the robot, the higher the redundancy and the higher the precision to accurately follow a given trajectory. By using the Fourier transform, it is possible to filter the small frequencies and the resulting trajectory will be followed with relatively low errors. This filtering process can be optimized depending on the application requirements, and supposes the removal of the smallest links of the robot while retaining the biggest ones.

- From a constructive perspective and since the theory of this methodology is purely kinematic and based on constant velocities, once the links of the robot are rotating, the energy transferred to the robot should only be directed to keep that velocity constant due to undesirable mechanical frictions. Thus, the resulting robots are very energy-efficient and can almost passively follow a given trajectory just relying in their morphology. Therefore, it can be deduced that this kind of systems would be quite interesting in microgravity or for space exploration purposes.

- If the trajectories are defined continuously and closed as a loop, the robot will perform smooth movements. However, if the points of the trajectory are defined with big distances or jumps between each other, something very singular and interesting will happen to the robot kinematics: the links of the robot will tend to become parallel providing an incredible force to the system in the tip as a propulsion mechanism. 


\subsection{Discussion}

An ideal hyper-redundant continuous or soft robot would be modular, lightweight, safe to interact, compliant with the environment, robust, accurate, with low cycle times, high repeatability, high resolution and high maximum bending angles to grant great dexterity. However, it is concluded that it is not possible to obtain all of these characteristics at once due to current technology and materials limitations. Thus, these characteristics should be adequately tuned depending on each application. However, it is possible to find a great balance or a specific optimization to complete a determined task.

It is also concluded that a distributed location scheme of the actuators throughout the robot body is usually unfeasible for redundant robots, unlike for traditional ones. It is an unsustainable design strategy because hyper-redundant robots have a lot of degrees-of-freedom, hence a lot of actuators. This large number of actuators in the robot body adds more weight to the structure, requiring more power to balance this extraweight, forcing to use even bigger actuators, and creating a vicious circle. On the other hand, distributed actuation schemes could be interesting in microgravity environments and for space exploration purposes.

Therefore, ideas based on external actuation such as those based on tendons, pneumatics or magnetic sources are usually recommended for in-earth applications. Regarding the latter, it is possible to use the magnetic sources as a mean to obtain clever designs such as passive damping mechanisms or remote, contactless actuation or even levitation.

Additionally, a remote actuation scheme can always be selected to allow a lighter design with further minimization possibilities. Related to this, choosing the right materials is also crucial to get the optimal dimensions. Flexible materials would lead to continuous or soft robots, instead of rigid ones, increasing the compliance but decreasing the payload, the robustness or the accuracy. Specifically, the form factor is crucial for the design of soft robots where the consistency of the materials should find a close compromise with the force exerted by the actuators in order to obtain the desired behaviour. Consequently, finding the right design will be highly dependent on the application and the environment. 


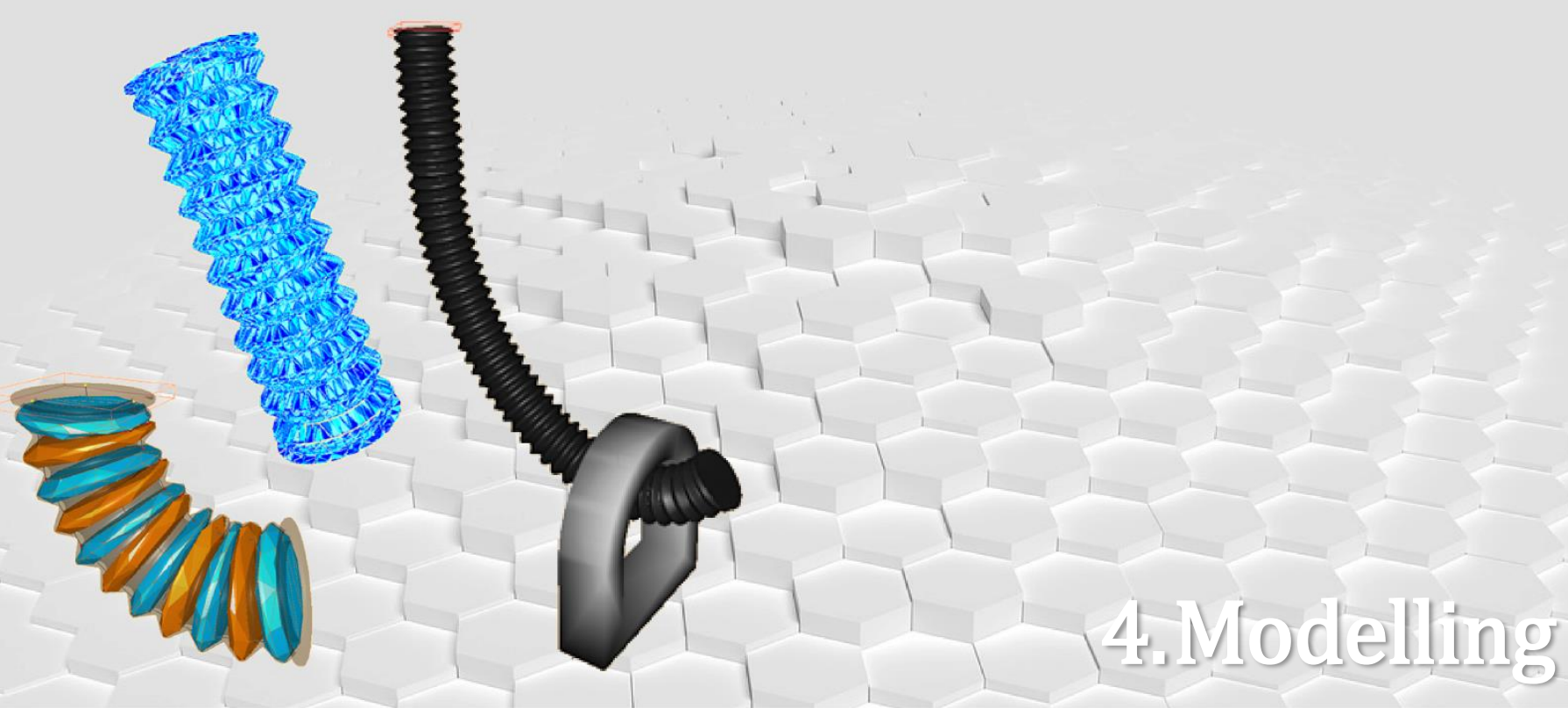

This section explains the implementation of up-to-date modelling techniques for discrete, continuous and soft robots. First ones are easy to model following rigid-body mechanics and their kinematic modelling can be performed using traditional conventions such as the Denavit-Hartenberg one. On the other hand, continuous robots, and especially soft robots are more difficult to model since they present numerous nonlinearities. Thus, a hybrid method that combines a geometric modelling technique with an on-board sensory system is first proposed. Additionally, modelling soft robots is a non-trivial task since their behaviours rely on their morphology, materials and surrounding elements. This section explains the challenging modelling process of a cable-driven soft robot called Kyma. For this purpose, the real-time Finite-Element Method (FEM) is applied using the Simulation Open Framework Architecture (SOFA). And two methods are implemented and compared to optimize the model efficiency: a heuristic one and the Model Order Reduction (MOR). Both models are also validated with the real robot using a precise motion tracking system. Moreover, an analysis of robot-object interactions is proposed to test the compliance of the presented soft manipulator. As a result, the real-time FEM emerges as a good solution to accurately and efficiently model the presented robot while also allowing to study the interactions with its environment.

Further information of this section can be found in an original previous publication ${ }^{13}$. Also, an explanatory of this video can be found in the Electronic Supplementary Material (Video S. 2).

${ }_{13}$ Andrés Martín-Barrio et al., Modelling the Soft Robot Kyma based on Real-Time Finite Element Method. Computer Graphics Forum, 2020. 


\subsection{Discrete hyper-redundant robots}

A sequence of displacements and rotations can easily and accurately define the pose of rigid-body mechanisms with distinguishable links. This procedure is very common and widely assumed when applying the so-called Denavit-Hartenberg convention for regular, non-redundant robots as well [3]. In order to apply this method accurately, it is important that the selected robot is made by rigid materials that cannot bend. However, both rotational and prismatic capabilities can be modelled by this method. The process to find the direct kinematics of serial manipulators by this method is summarized as follows:

- DH1: Enumerate the links beginning with 1 as the first mobile link in the chain, and end with $n$ as the last mobile link. The fixed base will be enumerated with a 0 .

- DH2: Enumerate each joint beginning with 1 as the correspondent to the first DoF and ending with $n$.

- DH3: Localise the axis of each joint. If it is rotational, the axis will be located around the pivot line. If it is prismatic, the axis will be located along the displacement.

- $\quad$ DH4: For a given $i$ from 0 to $n-1$ put the axis $Z_{i}$ over the joint $i+1$.

- DH5: Put the origin of the system in the base as $\mathrm{S}_{0}$ in any point of the axis $\mathrm{Z}_{0}$. The axis $\mathrm{X}_{0}$ and $\mathrm{Y}_{0}$ will be set following the right-hand rotation rule to obtain $\mathrm{Z}_{0}$.

- DH6: For Ifrom 1 to $n-1$, put the system $S_{i}$ in the intersection of the axis $Z_{i}$ with the perpendicular line of both $\mathrm{Z}_{\mathrm{i}-1}$ and $\mathrm{Z}_{\mathrm{i}}$. If both axis crossed, $\mathrm{S}_{\mathrm{i}}$ would be located in the intersection point. If both axis were parallel, $\mathrm{S}_{\mathrm{i}}$ would be located in the joint $i+1$.

- DH7: Put $\mathrm{X}_{\mathrm{i}}$ in the perpendicular line of both $\mathrm{Z}_{\mathrm{i}-1}$ and $\mathrm{Z}_{\mathrm{i}}$.

- DH8: Put $Y_{i}$ so it follows the right-hand rule for rotations with $X_{i}$ and $Z_{i}$.

- DH9: Set the system $S_{n}$ in the tip of the robot so $Z_{n}$ is in the same direction of $Z_{n-1}$ and $X_{n}$ is perpendicular to $\mathrm{Z}_{\mathrm{n}-1}$ and $\mathrm{Z}_{\mathrm{n}}$.

- DH10: Obtain $\theta_{i}$ as the angle needed to rotate around $\mathrm{Z}_{\mathrm{i}-1}$ so $\mathrm{X}_{\mathrm{i}-1}$ and $\mathrm{X}_{\mathrm{i}}$ are parallel.

- DH11: Obtain $d_{i}$ as the distance along $\mathrm{Z}_{\mathrm{i}-1}$ that would be needed to move $\mathrm{S}_{\mathrm{i}-1}$ so $\mathrm{X}_{\mathrm{i}}$ and $\mathrm{X}_{\mathrm{i}-1}$ are aligned.

- DH12: Obtain $a_{i}$ as the distance along $\mathrm{X}_{\mathrm{i}}$ in order to make the origin of $\mathrm{S}_{\mathrm{i}-1}$ and $\mathrm{S}_{\mathrm{i}}$ coincident.

- DH13: Obtain $\alpha_{i}$ as the angle around $\mathrm{X}_{\mathrm{i}}$ needed so the system $\mathrm{S}_{\mathrm{i}-1}$ is totally coincident with $\mathrm{S}_{\mathrm{i}}$.

- DH14: Obtain the transformation matrices ${ }^{\mathrm{i}-1} \mathrm{~A}_{\mathrm{i}}$, where:

$$
{ }^{i-1} A_{i}=\left[\begin{array}{cccc}
\cos \left(\theta_{i}\right) & -\cos \left(\alpha_{i}\right) \sin \left(\theta_{i}\right) & \sin \left(\alpha_{i}\right) \sin \left(\theta_{i}\right) & a_{i} \cos \left(\theta_{i}\right) \\
\sin \left(\theta_{i}\right) & \cos \left(\alpha_{i}\right) \cos \left(\theta_{i}\right) & -\sin \left(\alpha_{i}\right) \cos \left(\theta_{i}\right) & a_{i} \sin \left(\theta_{i}\right) \\
0 & \sin \left(\alpha_{i}\right) & \cos \left(\alpha_{i}\right) & d_{i} \\
0 & 0 & 0 & 1
\end{array}\right]
$$

- DH15: Obtain the transformation matrix that relates the base with the tip of the robot.

$$
\mathrm{T}={ }^{0} \mathrm{~A}_{1}{ }^{1} \mathrm{~A}_{2} \ldots{ }^{\mathrm{n}-1} \mathrm{~A}_{\mathrm{n}} .
$$

- DH16: The matrix T defines the orientation and the position of the tip of the robot referred to the base expressed as the $n$ joint variables. 


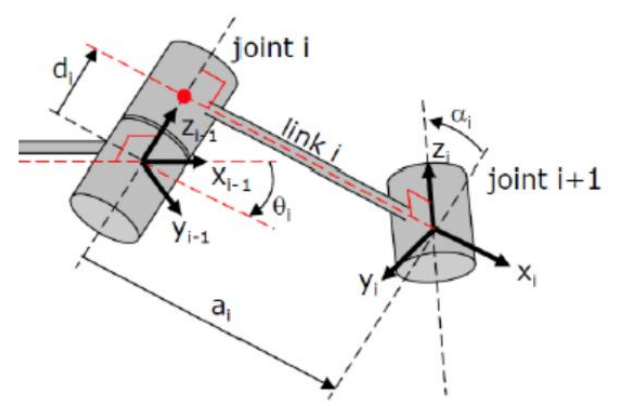

Figure 4.1. Denavit-Hartenberg convention to model rigid and discrete manipulators.

This traditional method will be further used in this document to model discrete and rigid manipulators. It is the most accurate and complete model to extract the kinematics even for hyper-redundant ones. If the dynamics should also be computed, further methods based on the Newton-Euler and Lagrange equations. However, this work will use the previously presented hyper-redundant discrete robot from Section 3.1, which moves at low velocities and accelerations for accurate inspection purposes. Thus, the dynamic forces can be neglected in this case, assuming an almost total kinematic system. 


\subsection{Continuous robots}

This work proposes a novel method to model continuous robots. As a remainder, a continuous robot is made by materials and structures that let the body to slightly deform and bend, usually accompanied by a higher dexterity. Therefore, previous described method cannot accurately capture these deformations since it assumes rigidity between links. In other words, continuous robots show non-linearities that have to be calculated in order to predict their movements with precision.

One of the most popular and easy methods to solve this problem has been already introduced as the Piecewise Constant Curvature (PCC). It assumes that the continuous robot bends following different sections that can fit as an arc of different radius circles. In reality, continuous robots are deformed by other forces such as the gravity, so the curves that compose their shape are often not constant. Thus, this method exhibits relatively high errors compared to others such as the Cosserat Rod, also introduced in Section 2.2. The Cosserat Rod allows to extract the non-linear bending capabilities of continuous robots with fairly relative low errors, taking into account twisting rotations and dynamic forces. However, it fails at capturing the interactions of the robot with the environment. In other words, if the continuous robot complies around an object, both the PCC and the Cosserat Rod method will fail to predict the pose of the robot. And the most important thing here is that continuous robots (as well as soft ones) are mainly used because of their flexibility and adaptability in terms of interacting with their surroundings. Thus, the state-of-the-art methods are considered as insufficient.

In this thesis, a hybrid method is proposed between a model and a sensory system. Models are usually created a priori in order to predict the robot movements even in absence of an accurate sensory system. However, the environment can be highly dynamic and changing. Motion capture systems such as an Optitrack $®$ camera setups can be very useful for testing in controlled environments but cannot constitute a portable and reliable solution. While on the other hand, sensors based on Fiber Bragg Grating (FBG) can allow such portability but they are also very sensitive for hazardous environments and expensive.

Thus, this work proposes to model continuous robots using the PCC method with an online correction of an Inertial Measurement Unit (IMU) array sensory system. For this purpose, the PCC method is firstly computed [162]. On the other hand, the robot is disposed with a set of IMUs provided with gyroscopes, accelerometers and magnetometers that grant the absolute rotation of the robot at a specific point in their body. The higher the number of IMUs in the array, the higher also the precision to obtain the pose of the robot. A multiplexor was also used to collect all the information from the IMUs, connected by the I2C communication. It is worth highlighting that in serial manipulators the error is accumulative from the base to the tip, so small deviations in each module can lead to high errors in the end-effector. This is why it is important to be very precise at every modelling step.
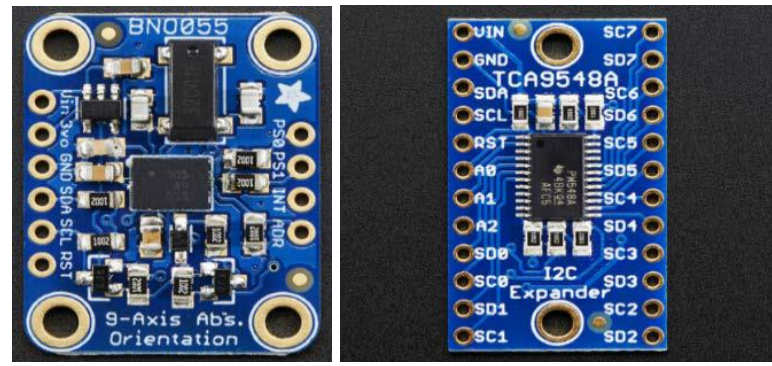

Figure 4.2. Sensor BNO055 and multiplexor TCA9548A. 
Then, the errors from the PCC were averaged and corrected by using the data from the sensory system. This method should be taken as a hybrid approach since it does not entirely predict the robot movements beforehand but corrects the pose with online information. Results were obtained at very high frequencies and reduced the relative errors to a $3 \%$, which is quite high compared to other state-of-the-art methods. Moreover, by using this sensory system, it is possible to capture the disturbances in the robot movements such as interactions with the environment or undesired collisions.

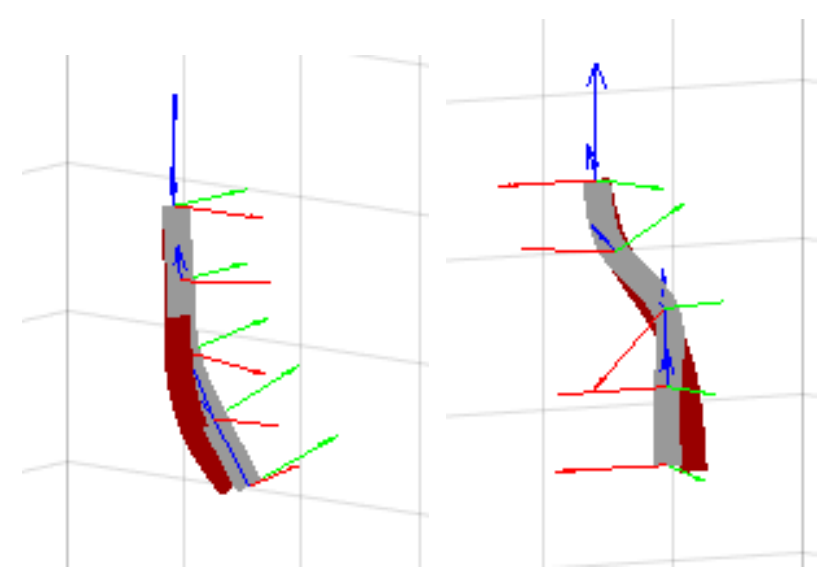

Figure 4.3. Experimental comparison of the real pose of the robot obtained with an Optitrackß system and the estimated pose of the robot with the presented method.

If the continuous or soft robot is actuated by tendons and has prismatic capabilities, as the one shown in Section 3.3, then the IMUs would grant the 3 DoF related to the orientation of a certain point, and the tendons could give information of the position of those points following the PCC scheme. This method is very cheap and quite easy to implement in any continuous robot. However, this method would not be feasible to predict the robot movements beforehand. Latest contributions to the state-of-the art show that, possibly, the method with more potential to model continuous and soft robots in real time is the real-time Finite Element Method (FEM) as discussed below. 


\subsection{Soft robots}

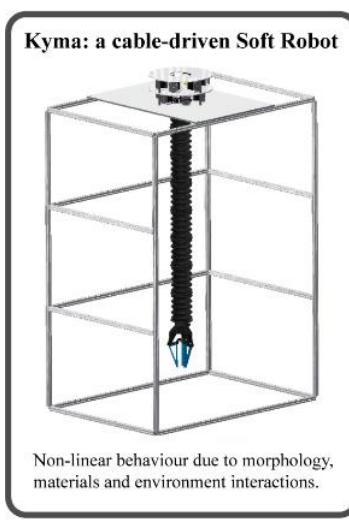

Figure 4.4. Main different stages to model the soft robot Kyma using the real-time Finite Element Method

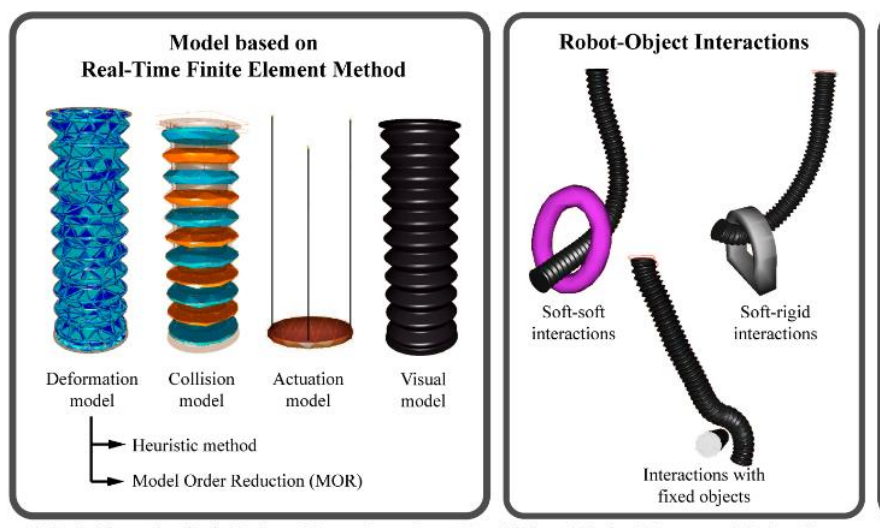

Modelling the Soft Robot Kyma based on Real-Time Finite Element Method

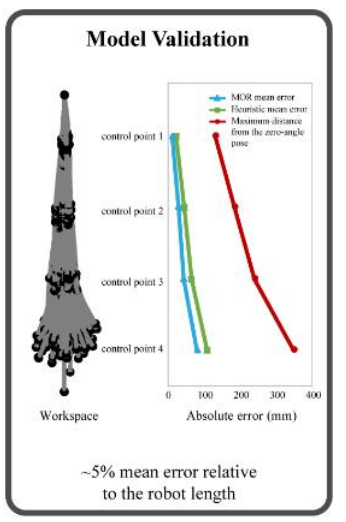

to the robot length

Soft robots can be understood as those programmable devices made from compliant materials, often inspired by nature, inherently safe to interact, and with high flexibility and adaptability skills.

This kind of robots can be considered a subset of continuous ones since they can continuously deform. In turn, continuous robots are often identified as a subset of hyper-redundant robots. Specifically, those underactuated, for having a large number of degrees-of-freedom, while only some of them being directly controlled.

Despite their complexity, soft robots are one of the most impacted research topics within the robotics scope because of their potential applications. They can be used in the medical field, especially for rehabilitation or in minimally invasive surgery procedures [241]; for exploration purposes [242]; in agriculture [243]; as personal robots to interact with people, or for entertainment, among others [244].

Typically, soft robots offer kinematic advantages as well as flexibility and safety against rigid ones. Also, they can be fault-tolerant, so they can correctly perform even if some of their actuators fail. Moreover, they exhibit better capabilities to move in complex environments with obstacles, compared to conventional robots [7]. However, research of such devices is often narrowed due to some engineering issues yet to be solved. For instance, it is important to still research about new technologies and materials to design and create these robots. Additionally, current modelling techniques are not efficient and accurate enough to provide a generalizable method for all kinds of soft robots and actuation. On the other hand, past efforts focused on solving the inverse kinematics model have not fully exploited the whole-body capabilities of these robots since only the end-effector was directly commanded. Therefore, once a feasible model is obtained, integrated control or teleoperation schemes are required to manage all the available degrees-offreedom.

Currently, there are two main strategies to solve the modelling issue for continuous and soft robots (Section 2.2). On one hand, model-free approaches rely on learning techniques based on artificial intelligence to construct underlying models from large datasets. On the other hand, model-based ones propose analytical or numerical methods for the same purpose. This work aims to test a numerical modelling strategy based on a Finite Element Method (FEM) using SOFA, an open-source library.

As a remainder, the soft robot Kyma will be used for this purpose. It is an original soft continuous manipulator designed in the Centre for Automation and Robotics (CSIC-UPM). Its name comes from the Greek and means wave. It is a long cable-driven robot whose structure allows prismatic and bending movements. Because of its morphology and dynamic properties, its behaviour is far from trivial. This is why 
a real-time Finite Element Method (FEM) will be proposed to accurately model the multiple deformations that cause the mechanical coupling between the actuators and the soft structure.

The main hypothesis of this work states that real-time FEM constitutes a reliable and generalizable solution to model soft robots. Subsequent sections will contrast this assumption.

Accordingly, the contributions of this section are the following. Firstly, it provides a description of a challenging modelling process of a soft manipulator. Specifically, this robot gathers several challenges: very thin-walls in a big robot, a strong dependence on its wavy morphology, the appearance of both bending and prismatic movements or a high number of actuators. A heuristic method and the Model Order Reduction technique are proposed to overcome these problems. Secondly, this work presents an analysis of robotobject compliance in different scenarios. Actually, soft devices are relevant due to their high interaction skills. Thus, models that do not study the synergies of these robots with their environment can be considered as incomplete. Finally, a validation scheme is proposed to contrast the goodness of the model compared to the real robot behaviour.

\subsubsection{Finite-Element Method model}

The Finite Element Method (FEM) is a useful tool that has been around for decades. Traditional FEM techniques are used in computers to solve differential equations associated with physical problems over complex geometries and with heavy computational costs. However, its use has become popular to model soft robots when it allowed applications in real-time [9].

First, it is very important to define real-time since it is commonly misused. A real-time system can be understood as an information processing activity that responds to an externally generated stimulus in a previously determined frame of time [245]. Thus, depending on the application, real-time requirements will be different. For this work, it will be considered that the model works in real-time if it provides enough outputs to take control actions at the actuators maximum speed requirements, as explained in Section 4.3.3.

The main justification to use this strategy for modelling the soft robot Kyma (Figure 3.7) relies on its behaviour, highly dependent on its wavy morphology, which has been designed to take advantage of its prismatic capabilities. While other methods can extract the non-linearities of soft robots, the FEM approach is the only one that deals with the morphology issue. Thus, it is the only method that does not neglect the geometrical design of soft robots. Additionally, and most important, while other state-of-the-art methods suppose the robot to be stiffer than its surrounding environment [9], the FEM strategy allows contact response and compliance analysis, as analysed in Section 4.3.2.

This model has been implemented by using the Simulation Open Framework Architecture (SOFA) [246], an open-source framework that performs FEM in real-time as well as constraint-based models. It has been used alongside the Soft Robots Plugin, which extends its capabilities to model continuum mechanics and boundary conditions using a unified framework based on Lagrange multipliers [174].

The main concepts of the SOFA Framework and FEM are explained in [174]. The system is based on the second law of Newton, that models the dynamic behaviour of a body as shown in Eq. 4.3.

$$
M(q) \dot{v}=P(t)-F(q, v)+H^{T} \lambda
$$

Here, $q \in \mathbb{R}^{n}$ is the vector of generalized degrees-of-freedom, the displacements of the mesh nodes; $v=\dot{q} \in$ $\mathbb{R}^{n}$ is their corresponding velocity vector; $M(q)$ is the inertia matrix; $F$ represents internal forces applied to the simulated object depending on the current state, such as the deformation law; $P$ collects other known external forces; $H^{T}$ is the matrix with the constraint directions; and $\lambda \in \mathbb{R}^{n}$ is the vector of Lagrange 
multipliers containing the constraint force intensities, such as the force applied by actuators like tendons or pneumatic pressures.

This system is solved by methods oriented to ordinary differential equations, such as the implicit backward Euler scheme [174]. From this, it is possible to infer the state variables $q$ and $v$ using a matrix solver. As a result, the element's force fields, masses, contacts and constraints give place to the position and velocity outputs.

On the programming interface, SOFA makes use of different nodes and components to extract the features and behaviours of the robot to create a model. The principal implementation of the FEM model relies on these components, so they will be further explained. First, the volumetric mesh that describes the arrangement of the small elements for computing the robot deformations will be described (Section 4.3.1.1). Next, the tendon-based actuation system will be defined (Section 4.3.1.2). Also, a collision model will be included for two main purposes: to recreate the robot movements caused by the interaction of the bellow folds and to analyse its compliance with external objects (Section 4.3.1.3). Finally, the visual model will provide the external appearance of the soft robot (Section 4.3.1.4).

To clarify the implementation, a scheme with the main components to construct the model is proposed in Table 4.1. Also, to facilitate the understanding of this section, the main features of Kyma are presented in Table 4.2 .

\begin{tabular}{|c|c|}
\hline Root node & \\
\hline -RequiredPlugin & SoftRobots plugin \\
\hline -RequiredPlugin & SofaPython plugin \\
\hline -RequiredPlugin & SOFAMiscCollision plugin \\
\hline Visualstyle & Visual appearance of the scene \\
\hline FreeMotionAnimationLoop & Constraint solver \\
\hline GenericConstraintSolver & Constraint solver \\
\hline BruteForceDetection & Collision detection by pair-wise tests \\
\hline -LocalMinDistance & Set distances to consider collisions \\
\hline Kyma node & \\
\hline -EulerImplicit & Time integrator \\
\hline -MeshGmshLoader & Mesh loader for Gmsh file format \\
\hline Mechanicalobject & Mechanical state vectors \\
\hline UniformMass & Define the element's mass \\
\hline -TetrahedronFEMForceField & Tetrahedral finite elements \\
\hline -RestShapeSpringsForceField & Elastic springs used to anchor the robot \\
\hline Kyma visualization node & \\
\hline OgLModel & Visual model for OpenGL display \\
\hline BarycentricMapping & Mapping using barycentric coordinates \\
\hline Kyma collision model & \\
\hline MeshSTLLoader & Mesh loader for STL file format \\
\hline Mechanicalobject & Mechanical state vectors \\
\hline Point & Collision model based on points \\
\hline Line & Collision model using a linear mesh \\
\hline -Triangle & Collision model using a triangle mesh \\
\hline BarycentricMapping & Mapping using barycentric coordinates \\
\hline Disks node ( $x 4$ units) & Simulation of the inter-modular disks \\
\hline Tendons node (x12 units) & Actuation cables anchored in the disks \\
\hline Mechanicalobject & Mechanical state vectors \\
\hline CableConstraint & Tendons used to actuate the robot \\
\hline BarycentricMapping & Mapping using barycentric coordinates \\
\hline PythonScriptController & External code source based on python \\
\hline Obstacles (optional) & Obstacles for interacting purposes \\
\hline
\end{tabular}

Table 4.1. Main node hierarchy and a bullet list of the principal components to model Kyma in the SOFA environment alongside their descriptions. 


\begin{tabular}{lll}
\hline Variables & Real values & Heuristic tuning \\
\hline Exterior diameter & $100 \mathrm{~mm}$ & \\
Total length & $1200 \mathrm{~mm}$ & $10 \mathrm{~mm}$ \\
Thickness & $2 \mathrm{~mm}$ & \\
Material & PVC & $2.5 \mathrm{~kg}$ \\
Density & $1160 \mathrm{~kg} / \mathrm{m} 3$ & \\
Mass & $1.10 \mathrm{~kg}$ & \\
Young's Modulus & $10 \mathrm{~N} / \mathrm{mm} 2$ & \\
Rupture strain & $400 \%$ & \\
Actuated sections & 4 & \\
Number of tendons & 12 &
\end{tabular}

Table 4.2. Main parameters of Kyma to construct the FEM model and a heuristic adjustment to decrease the computational cost.

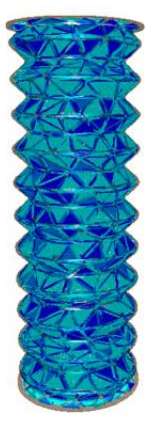

(a)

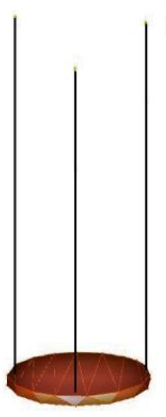

(b)

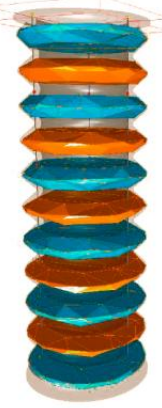

(c)

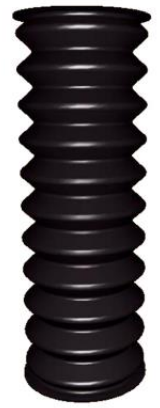

(d)

Figure 4.5. Main components to model in the SOFA environment. (a) Deformation model. (b) Actuation model. (c) Collision model. (d) Visual model.

\subsubsection{Deformation model}

The first and maybe most important step to compute the FEM model is also known as the volumetric mesh generation. It is based on selecting an adequate shape and size of the small elements that are going to compute the macroscopic deformations of the robot. Those shapes could be either cubes, dodecahedra or even two-dimensional elements among others. For this work, a tetrahedron shape has been selected for being the best to fit in generalized volumes (Figure 4.5a). The deformation model is the most important one since the actuation, collision and visual models will adopt its pose using a Barycentric Mapping.

The mesh resolution and accuracy are always balanced in every Finite Element Analysis to obtain reliable results with an appropriate number of elements. In other words, the higher the number of elements, the better the accuracy and the higher the computational time. It is possible to find more information about the relation of these variables in [247]. This last parameter, the computational time, is critical for this application because the model is needed to work in real-time. Thus, the number of elements of the mesh should be carefully set. Based on previous works, it is possible to establish that the SOFA Framework begins to perceive relative frame-rate decreases for more than 2000 elements, depending on the computer performance. Therefore, the deformation model of a soft robot should not exceed that value.

There are a lot of morphologies that accept several simplifications in the volumetric mesh to make it suitable for real-time applications. But Kyma, as well as a lot of soft robots, they are often designed very slim and hollow in order to be pressurized with air or to grant more flexibility. Because of this thin-thickness structure, the number of tetrahedra to fit the volume must be very high (Figure 4.6a). In this work, two different solutions are applied. Firstly, a heuristic method proposes to tune a model with fewer elements to match the behaviour of the real robot (Section 4.3.1.1.1). Secondly, a Model Order Reduction technique [247] uses a set of offline simulations to reduce the number of state variables of the model (Section 4.3.1.1.2). 


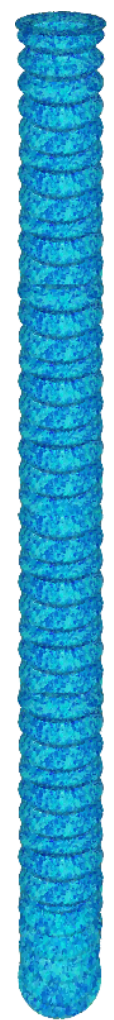

(a)

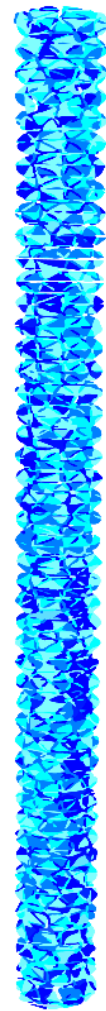

(b)

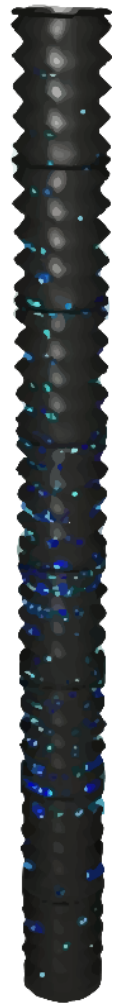

(c)

Figure 4.6. (a) Full-order deformation mesh with 120000 tetrahedra, infeasible for real-time applications. (b) The heuristic method adapts a model with 1650 elements to match the real robot behaviour. (c) The Model Order Reduction decreases the number of state variables of the model to 1886 while retaining its original characteristics.

\subsection{Heuristic method}

The following heuristic method is aimed to tune the characteristics of the robot by trial and error to match the behaviour of Kуma. To simplify the model, the initial Computer-Aided Design (CAD) resolution is decreased without substantially affecting its morphology. This lets the number of tetrahedra decrease from 120000 to 11000 . A considerable reduction but still, insufficient. After some observations, it is concluded that the limiting factor is the robot thickness, which is just $2 \mathrm{~mm}$. This forces to have a very high number of interconnected elements. Therefore, the thickness of the walls needs to be increased in order to decrease the number of elements and clarify whether or not it is feasible for real-time applications. The model similarity $(S)$ will be obtained by a min-max normalization of the evaluated thickness $(r)$ compared to the real one $\left(r_{\min }=2 \mathrm{~mm}\right)$ and the maximum thickness $\left(r_{\max }=50 \mathrm{~mm}\right)$, as shown in Eq. 4.4. The results are collected in Table 4.3 .

$$
S=100 *\left(\frac{r-r_{\max }}{r_{\min }-r_{\max }}\right)
$$

\begin{tabular}{lcl}
\hline & Model thicknessNumber of tetrahedra & Similarity \\
\hline $2 m m$ (real) & 11000 & $100 \%$
\end{tabular}




\begin{tabular}{lll}
$3 \mathrm{~mm}$ & 9000 & $98 \%$ \\
$5 \mathrm{~mm}$ & 2500 & $94 \%$ \\
$10 \mathrm{~mm}$ & 1650 & $83 \%$ \\
$50 \mathrm{~mm}$ (solid) & 600 & $0 \%$ \\
\hline \multicolumn{2}{c}{ Table 4.3. Analysis of the model thickness versus the number of FEM elements. }
\end{tabular}

The higher the model thickness, the less similarity to the real robot but also the smaller computational cost. It is very important for this type of applications to find a compromise between the number of elements while, at the same time, to find an accurate model and replicate the real robot behaviour. The real-thickness model is not suitable because the computational cost is not feasible. On the other hand, a completely solid model is not representative of the robot's behaviour. In this case, a model of $10 \mathrm{~mm}$ thickness has been heuristically selected to be the one with the best compromise between similarity and feasibility as shown in Table 4.3. Due to the differences of this model with the real robot, further experimental adjustments in the mass is considered in order to reproduce the real behaviour while still maintaining the main morphology (Figure 4.6b). With this strategy, the robot characteristics are not perfectly retained, but the macroscopic deformation properties are quite similar.

About the implementation, first, an EulerImplicit component is created to provide solutions to the underlying differential equations for the deformation model. Then, the mesh should be loaded from a $m s h$ extension file. To create the mesh, it is needed to use third-party programs such as gmsh. Also, MechanicalObject and UniformMass components are added to provide dynamic properties to the deformation model node. Next, the TetrahedronFEMForceField component implements a co-rotational FEM method based on tetrahedra. To finish, the robot should be anchored in its base to avoid its fall due to the gravity action. For that purpose, the BoxROI is used to define a region of interest, in which the RestShapeSpringsForceField component will restrict the environment forces.

\subsection{Model Order Reduction}

Previous heuristic methods can be useful to adjust the behaviour of the model to a particular soft robot. However, they do not constitute a general or reliable solution for the modelling process of such devices. On the other hand, Model Order Reduction (MOR) techniques retain the exact robot characteristics and use a set of offline simulations to reduce the number of state variables of the model. More information about this strategy can be found in [247].

For this process, it is necessary to ensure that the accuracy of the full-order model is representative of the real soft robot. Successively, evaluate the number of actuators and their range. Then, define a number of samples within each actuator range. If it is feasible to compute an exhaustive process, then the state variables will be stored. Otherwise, a non-exhaustive strategy should be selected like the Latin Hypercube or the Bayesian. Finally, the reduced basis is defined by performing a Proper Orthogonal Decomposition (POD). Some works even used this kind of reduced-order models and applied dynamically closed-loop architectures [248].

Regarding the implementation, the ModelOrderReduction plugin is used, the scene is prepared and the objects to animate are defined. About the actuators, their increment in each animation is set to a $20 \%$ of their total range, and the time between animations is $5 \mathrm{~s}$. Additionally, the presented robot has big prismatic capabilities; it can shorten up to $25 \%$ of their full length. Consequently, this will also determine the range of the actuators. Finally, the level of accuracy to select the reduced basis is set to $0.1 \%$ (Figure 4.6c). 


\subsubsection{2. $\quad$ Actuation model}

As explained in Section 3.3, the actuation system of Kyma (Figure 4.5b) is based on 12 equispaced tendons around a circle inside the robot cavity. They are actuated by a set of 12 synchronized stepper motors. These cables are distributed in groups of 3 for each section. Combinations of displacements for this arrangement allow the robot to rely on 3 controlled degrees-of-freedom for each section, two rotational and one prismatic (Figure 4.7). Thus, the whole robot has a total of 12 controlled degrees-of-freedom, although similar to the rest of soft robots, they can be considered continuous and underactuated for having an infinite number of virtual degrees-of-freedom as well.

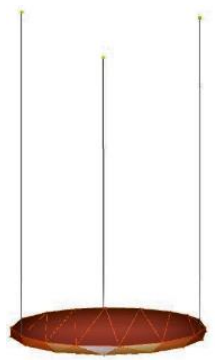

(a)

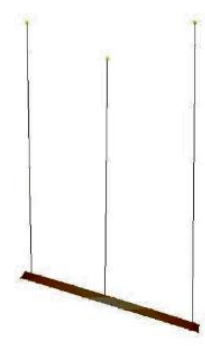

(b)

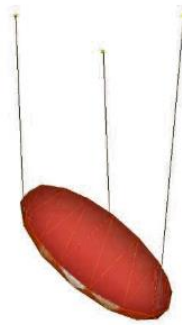

(c)

Figure 4.7. Actuation system based on three tendons per section. (a) Not actuated, (b) side view of the left-tendon displacement and (c) multiple-tendon displacement.

This set of tendons are virtually defined to exert displacements from a prefixed pull point to an anchor point. However, they are not capable to collide throughout their way. For this reason, they are guided through a set of intermediate points consistent with the deformation model movements (Figure 4.8).

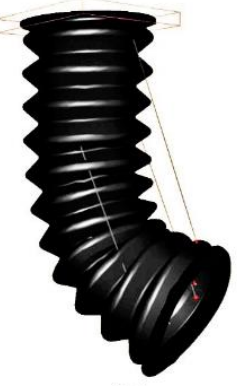

(a)

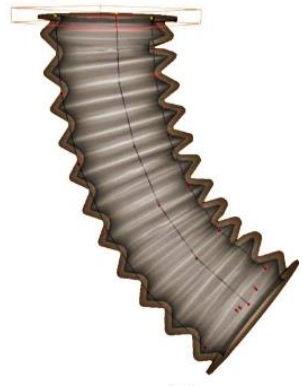

(b)

Figure 4.8. Transmission system based on (a) tendons without guidance and (b) the same system restricted to the robot morphology.

The complete model for the actuation system is represented in Figure 4.9, where a set of displacement values results in one configuration for the deformation model. 


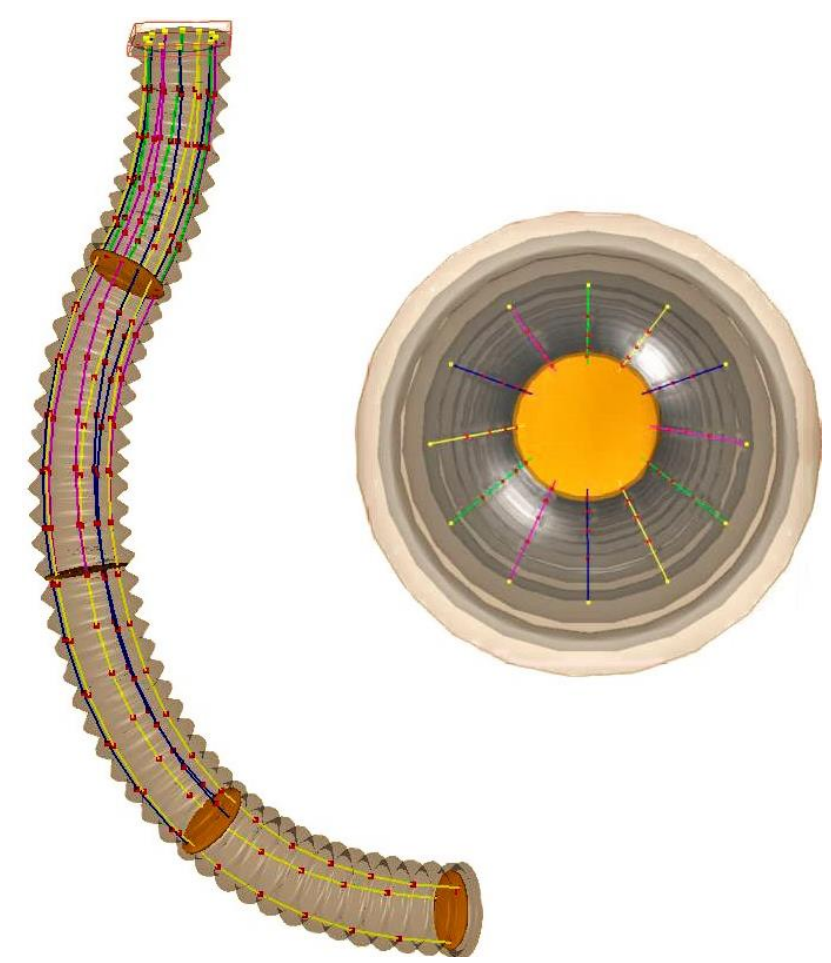

Figure 4.9. Side and top view of the complete actuation system of Kyma. Tendons corresponding to the same robot section are represented with same colours.

According to the SOFA implementation, a MechanicalObject component stores the values for each cable. All of them are then defined as CableConstraint components with information relative to their lengths, guides, anchorages, or pull points among others. This data is mapped to compute their correspondent to the deformable body using the BarycentricMapping object. The main controlled variable will be the length of each tendon, allowing the robot to be moved. For this purpose, a PythonScriptController component will be in charge of coordinating the actuators.

\subsubsection{Collision model}

The collision model (Figure 4.5c) has been implemented for two main purposes. On one hand, the robot must self-collide: the bellows' folds let the robot be compressed and elongated, but they should also interact with each other to precisely recreate the real robot behaviour. On the other hand, the collision model should also let the robot interact with external objects as will be explained in Section 4.3.2.

The same collision model design is created for both purposes in terms of reducing the computational cost. If the self-collisions were not considered, the robot deformation model would overlap the bellows in a nonrealistic way (Figure 4.10b). In this context, it is recommended to deal with each collision system independently for better results. Thus, if the soft robot is made with 8 bellows provided with 6 folds each, it will be expected to have 48 independent collision models to consider all the possible permutations. However, it was experimentally deduced that interactions between neighbour folds are enough to recreate the robot behaviour (Figure 4.10a). 


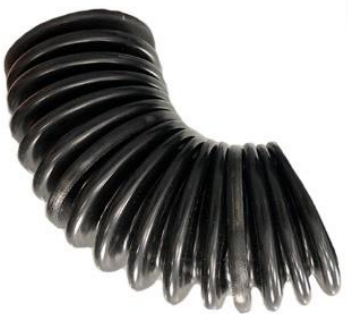

(a)

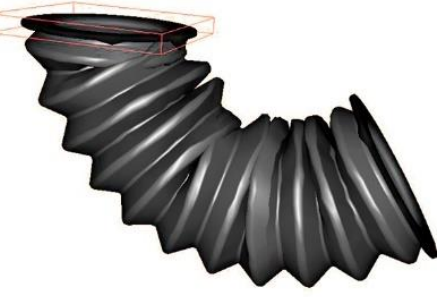

(b)

Figure 4.10. Difference between (a) the real robot behaviour with physical restrictions and (b) the undesired model without selfcollision restrictions.

Based on this, the initial collision model was reduced to a set of 2, instead of 48, as shown in Figure 4.11. Consequently, the computational cost is widely reduced while still obtaining good results. To define the collision system, first, it is needed to assign each one of them to different groups.

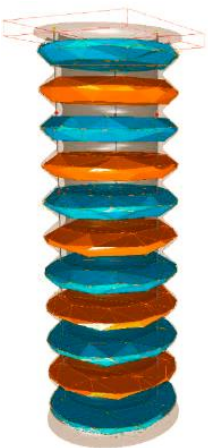

(a)

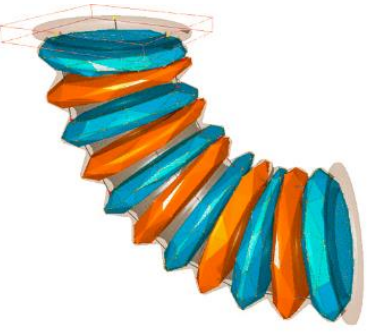

(b)

Figure 4.11. Self-collision restriction model for one section. (a) Robot in rest pose. (b) Actuated robot. Different colours are used to depict different collision systems. This model describes better the real robot behaviour.

There are other simpler collision systems available in the SOFA Framework such as those two-dimensional based on point-plane distances. These strategies seem to work well to prevent self-collisions since interactions occur through the relative normal direction of the planes. However, this arrangement is not appropriate to avoid collisions with external objects, which would usually approach from the tangential directions of the planes. Thus, the previously explained method, which is based on three dimensions to prevent self-collisions, is also useful to study the robot compliance with its environment as sown in Section 4.3.2.

In regards to the software implementation, the first component to explain is called LocalMinDistance. It enables setting the value of the distance at which two collision models are considered to be approaching or contacting. Then, both collision models should be loaded, typically from a two-dimensional file such as STL, using the MeshSTLLoader component. Next, the collision models are included in different groups through the Point, Line and Triangle components. All the elements within the same groups will not collide. Finally, a BarycentricMapping component is created to move the collision model with the deformation one. 


\subsubsection{Visual model}

The visual model (Figure 4.5d) is the most trivial node of the system since it is used to represent the real appearance over the deformation model, which is the one that truly computes its movement. Because of this, the visualization node is created as a Child of the deformation node. First, this visualization model is loaded with a OgIModelcomponent and its material, colour or shininess are tuned, among other parameters. Then, a BarycentricMapping component is again used to move this model according to the deformation one in real-time. The final effect looks like the visual model is the one moving, while the truth is only used for representation.

\subsubsection{Robot-object interaction}

Soft robots stand out against other types for being flexible, versatile and safe to interact. In Section 4.3.1, the FEM strategy has been used to model the soft robot Kyma. However, this approach can be used as well to model external objects, provided with a collision model, to study robot-object interactions. In this section, these synergies between the soft robot and its environment will be analysed.

It is important to fully understand what compliance means in the soft robotics scope. It can be understood as the act of conforming, acquiescing or yielding. Conventional robots are traditionally controlled to properly locate its end-effector for a determined task. Soft robots expand that perspective. Thus, control actions over soft robots are usually taken over their whole-body because it can serve as an interface with the environment. For example, compliance allows soft robots to surround objects without risks of being damaged, facilitates the avoidance of obstacles for path planning operations, or allows them to anchor to different shapes and sustain their own weight.

In this context, the performance of Kyma under different scenarios has been tested. For example, it was studied how the robot interacts with rigid and static objects such as its supporting structure (Figure 4.12). It can be deduced that the robot begins to bend adapting its flexible body around the structure on its end. However, contact forces are also transmitted through the entire robot's macroscopic pose in a very realistic manner.
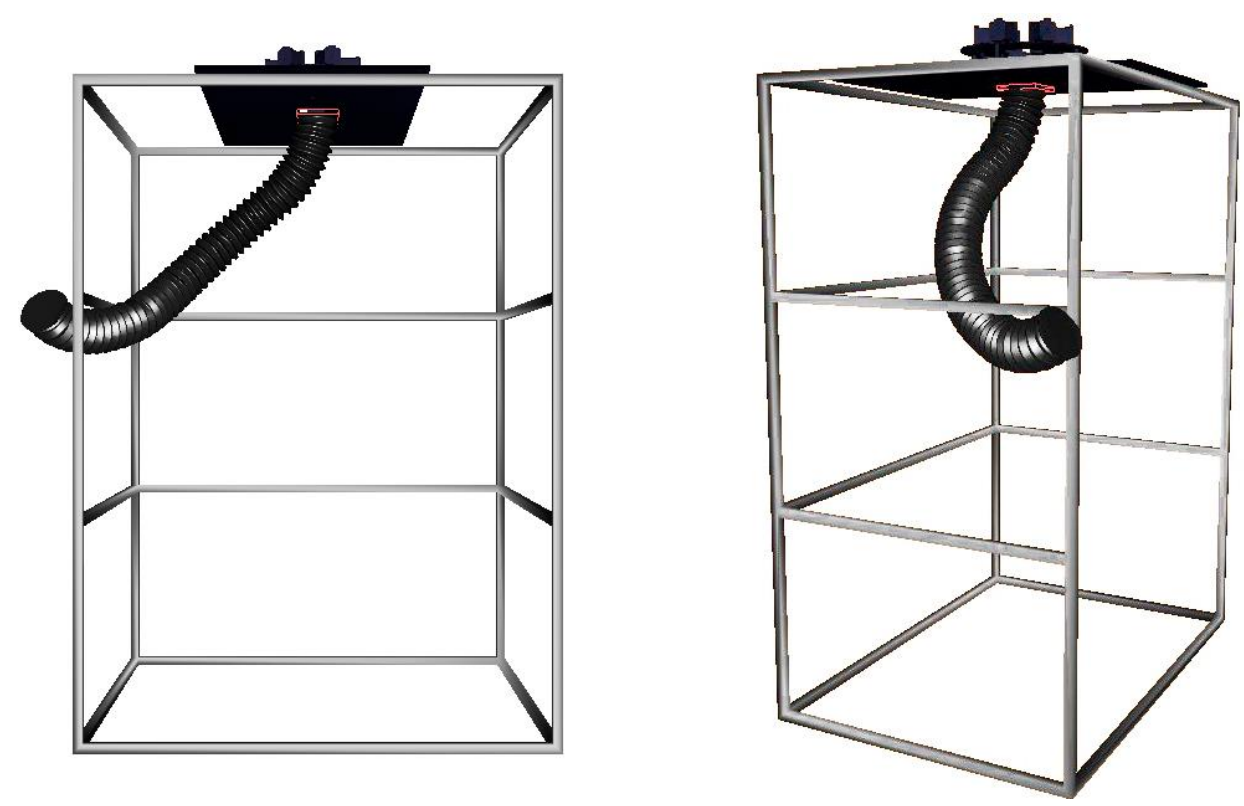

Figure 4.12. Front and perspective view of the Kyma model interacting with its own supporting structure in a zero-gravity environment. 
This is also represented in Figure 4.13, where the soft robot movement is intercepted by a rigid and fixed cylinder. The robot is also capable of gently surrounding this object, adapting its pose.
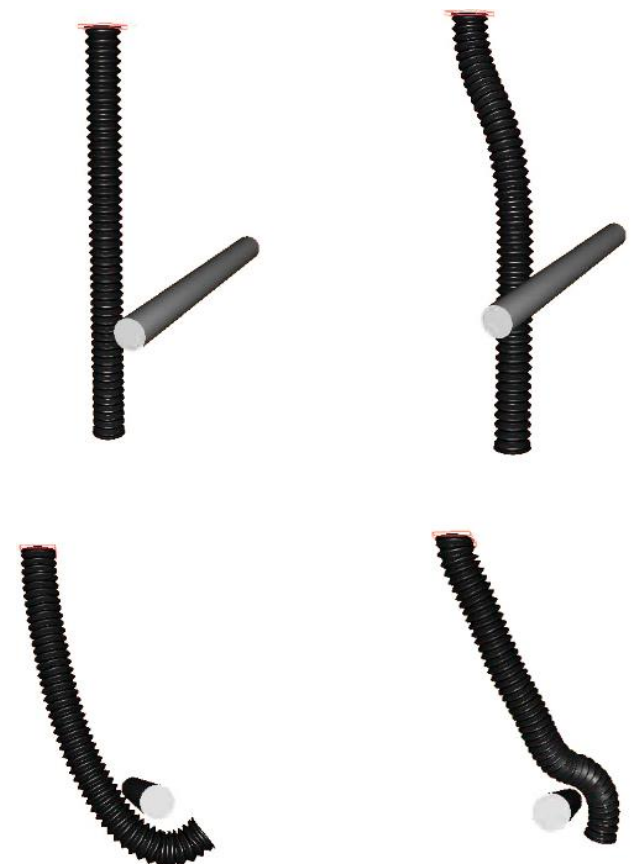

Figure 4.13. Soft robot compliance with a fixed obstacle in different situations.

It is important to clarify that these interactions also affect the actuation system, where new control actions can be inferred in real-time from possible contact events. Thus, modelling the environment constitutes an indissoluble union to the soft robot model itself. Both models should not be understood as different systems but as a whole since obstacles can directly alter the robot movement. As previously mentioned, models that do not study the environment as part of the soft robot itself can be presumed as incomplete since these robots are designed for such purposes. In this context, it is even possible to add or modify scene parameters to alter the real robot behaviour such as virtual obstacles or forces, to make tests prior implementation of the robot in a real scenario.

Additionally, as Figure 4.14 depicts, it is possible to make interactions with deformable objects in the same manner. Here, the robot configuration exhibits high compliance with the obstacle. Its movements are less altered than in previous situations such as in Figure 4.13. In all of these cases, the robot parameters such as mass or those related to deformations like Young's modulus, are strongly coupled with the external object ones. 

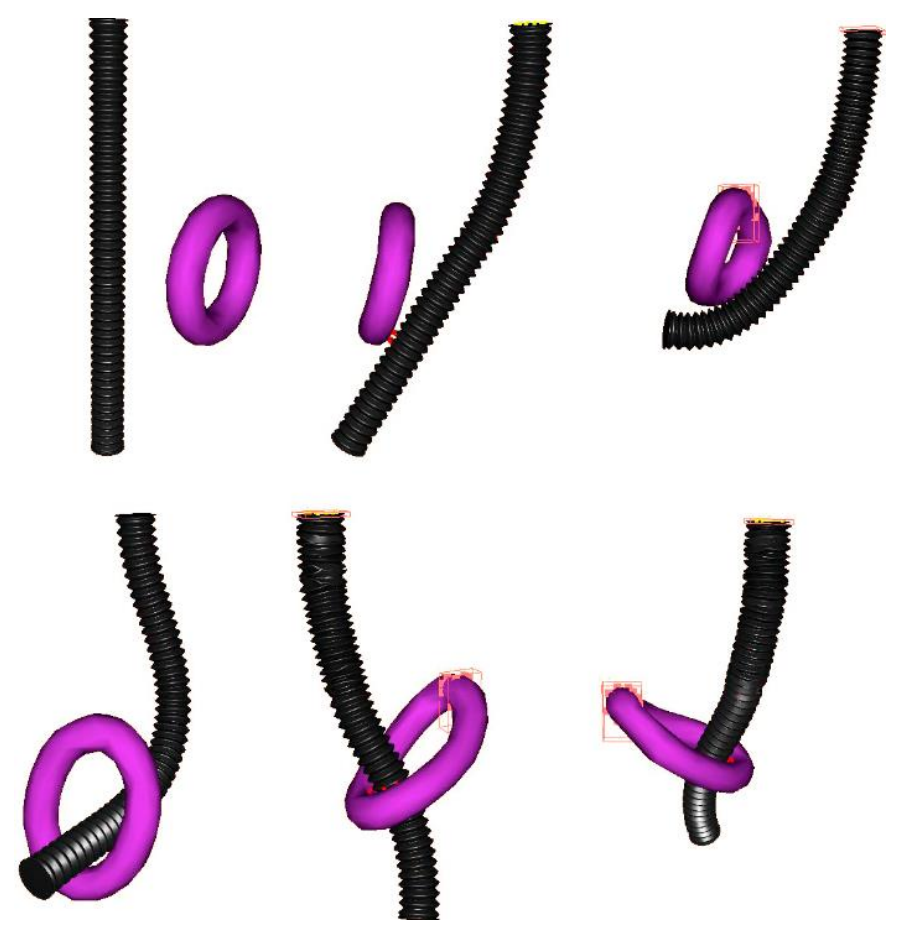

Figure 4.14. Kyma interacting with a flexible toroid-shape object.

In the latter experiment, Kyma was expected to lift a rigid, non-fixed object from the ground (Figure 4.15). In this case, a favourable outcome depends on the object mass and shape. Nevertheless, under the right conditions, it was proven that the soft robot is able to perform manipulation operations using its own body.

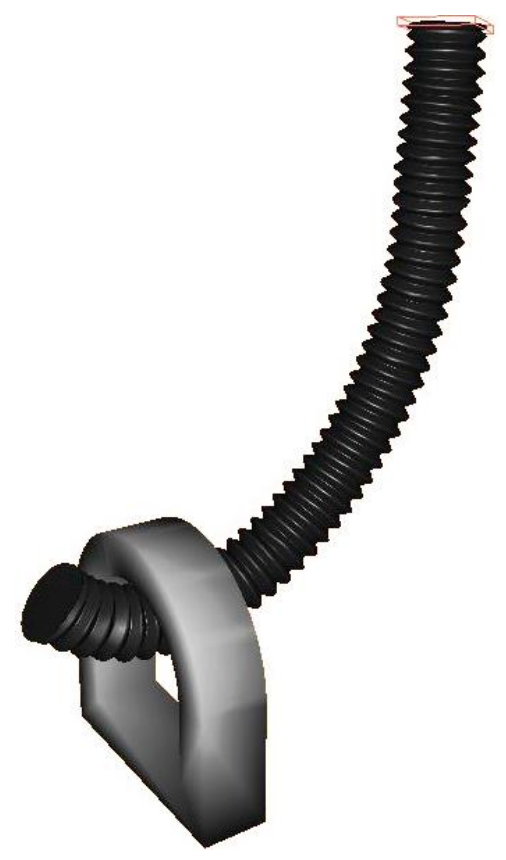

Figure 4.15. Lift of a non-fixed object from the ground using the robot body. 


\subsubsection{Model validation}

Both previously explained models, the heuristic and the Model Order Reduction one, have been tested by making comparisons with the real robot behaviour. Two metrics have been studied to analyse the model feasibility: computational time and accuracy.

About the first metric, the real-time capability has been tested by actuating the model at a slightly higher speed than the prefixed motors maximum speed $(120 \mathrm{rpm})$. Under these circumstances, the model provides its outputs at an adequate frequency of $20 \mathrm{~Hz}$ to compute the robot dynamics. This value may not be just dependent on the number of elements of the deformation model, but also on the computer system, the collision model, or the visual one, among other parameters. In fact, the wavy morphology of the robot Kyma influences the computational cost of the collisions management. The following tests were conducted in a computer with Intel CoreTM i7-8700 at 3.70GHz, x64 architecture and 16Gb of RAM. Higher frequency rates could be obtained using a computer with better specifications.

Regarding the accuracy, both the soft robot Kyma and the real-time FEM models have been equally actuated to compare their whole-body configurations. For that purpose, a set of 100 random robot poses has been compared to evaluate the model accuracy within its entire workspace. To measure the exact pose of Kyma, an OptiTrack motion tracking system has been used. Thus, several passive markers made of retro-reflective material have been deployed on the robot's surface to capture its shape by using an array of infraredsensitive cameras and light sources (Figure 4.16). The motion capture system works at 250 frames per second and its accuracy is below $0.1 \mathrm{~mm}$. Because of this, the obtained results by the motion tracking system can be considered as the ground truth.

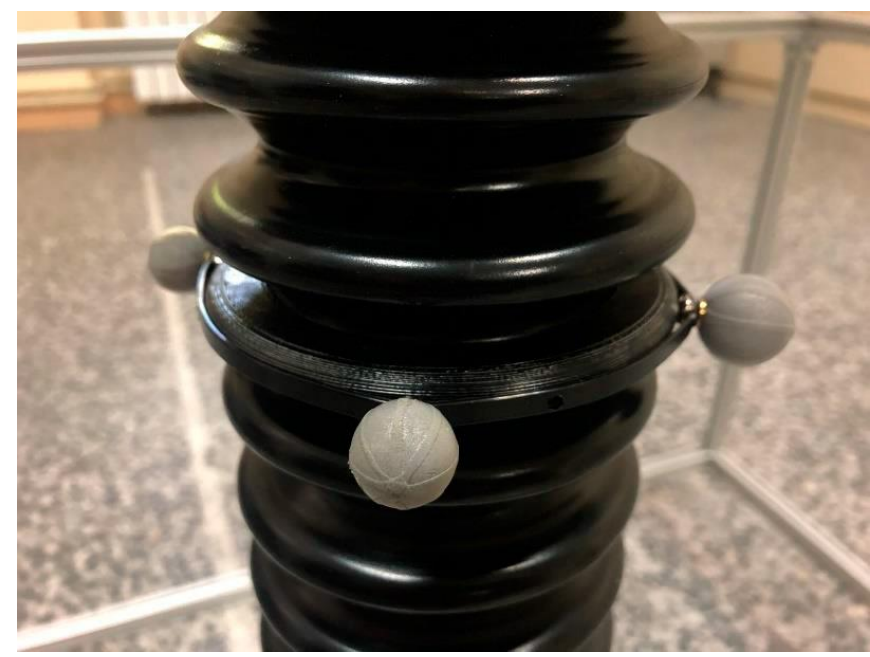

Figure 4.16. Three of a total of twelve infrared-sensitive passive markers, used by the motion capture system to obtain the robot pose.

Then, both the robot and the FEM models are equally actuated as shown in Figure 4.17. If all the tracked poses are combined in the same graphic, it is possible to show the explored workspace (Figure 4.18). Exact results are exhibited in Table 4.4. Here, the mean, minimum and maximum errors are calculated and can be analysed concerning their maximum distance from the robot's zero position. 

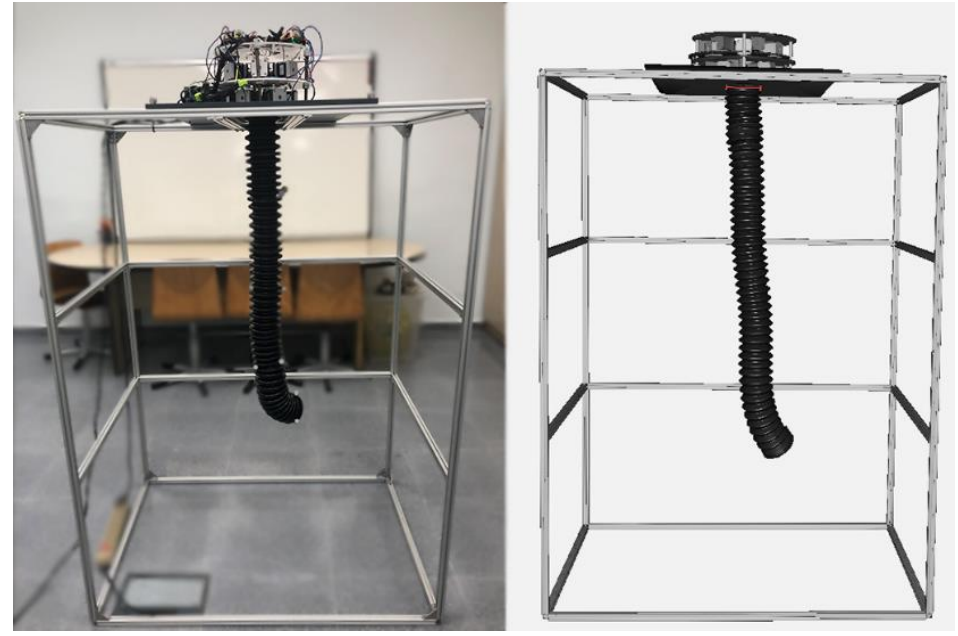

Figure 4.17. Comparison of a single test of the real robot (left) versus the obtained model (right).

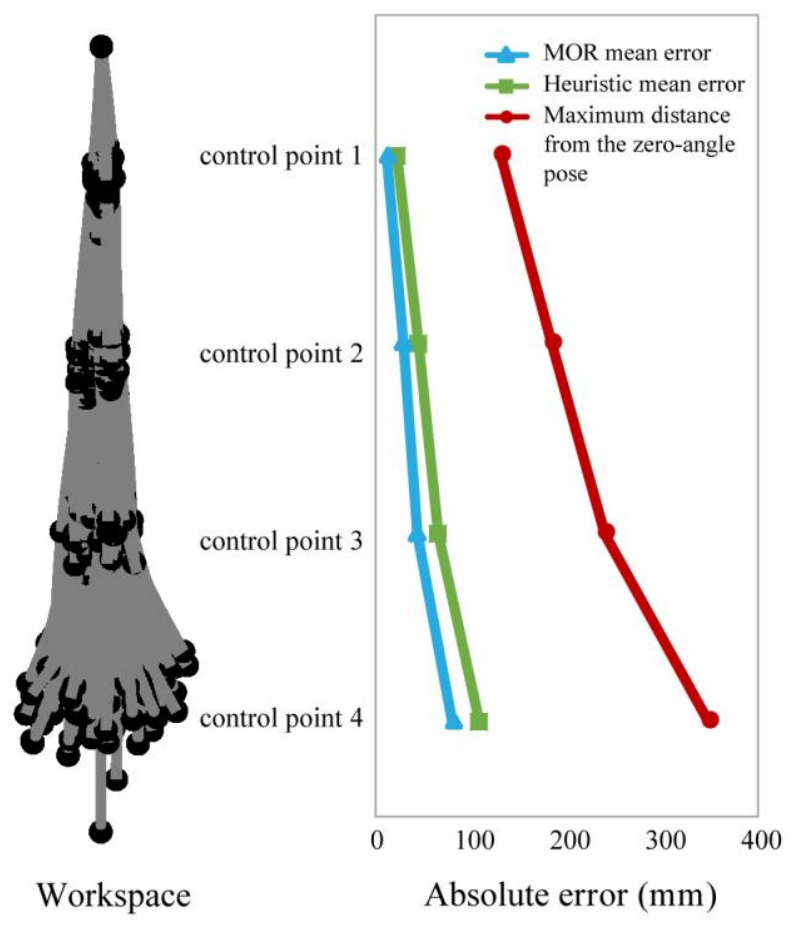

Figure 4.18. Real robot workspace representation for 100 iterations and validation of the FEM model. The absolute error is compared to the Euclidean maximum distance of the robot from a non-actuated pose.

\begin{tabular}{ll|lllll}
\hline Control point & $h$ & $D_{\max }$ & $\bar{x}_{1}$ & $\bar{x}_{2}$ & $x_{\min }$ & $x_{\max }$ \\
\hline 1 (base) & 300 & 130 & 22 & 20 & 3 & 66 \\
2 & 600 & 183 & 44 & 40 & 6 & 89 \\
3 & 900 & 239 & 64 & 59 & 10 & 124 \\
4 (tip) & 1200 & 348 & 107 & 99 & 46 & 195 \\
\hline All: & - & 348 & 59 & 56 & 3 & 195
\end{tabular}

Table 4.4. Validation results of the FEM Model for 100 random pose iterations. Actuated control points are numbered from the base to the tip as 1 to 4 respectively. h: distance of the control points to the base. $D_{\text {max }}$ : maximum separation of the control points from the zero-angle pose. $\bar{x}_{1}$ : mean error using the heuristic method. $\bar{x}_{2}$ : mean error using the Model Order Reduction method. $x_{\min }$ : minimum error. $x_{\text {max }}$ : maximum error. Errors are accumulated from control points 1 to 4 . Units in millimetres. 
It is important to clarify that the main purpose of soft robots relies on easily adapting or safely interacting with their environment. However, not precise enough models can result in an infeasible closed-loop control. Thus, accuracy must be studied in soft robot models but it should not be evaluated with the same yardstick as for rigid ones.

It can be seen in Table 4.4 that the heuristic model locates its first control point with an accuracy of $22 \mathrm{~mm}$ on average while getting maximum ones up to $3 \mathrm{~mm}$. Similarly, the MOR model obtains a mean accuracy of $20 \mathrm{~mm}$ for the first control point. Likewise in all serial manipulators, the error is accumulated from previous to subsequent control points. Thus, the tip gets higher errors than those control points close to the base. Additionally, the MOR model presents a slightly higher accuracy than the heuristic one, but both of them are quite similar. The MOR technique relies on the characteristics of the real robot and proposes a general solution without any human intervention. However, the heuristic method is less reliable since it highly depends on the robot and on a specific decision-making process.

Taking into account the Euclidean maximum distance of each control point from their non-actuated state, it is possible to infer the relative error. In similar works, such as [9], a $1.4 \mathrm{~mm}$ mean error is obtained using the same framework for a smaller robot that works in a workspace of $32 \mathrm{~cm}^{3}\left(4.37 \cdot 10^{-2} \mathrm{~mm}\right.$ per $\left.\mathrm{cm}^{3}\right)$. However, the current work obtains mean errors of $59 \mathrm{~mm}$ for the heuristic model and $56 \mathrm{~mm}$ for the MOR one within a workspace of $152000 \mathrm{~cm}^{3}$ (around $3.75 \cdot 10^{-4} \mathrm{~mm}$ per $\mathrm{cm}^{3}$ ). Thus, the presented models presents 2 orders of magnitude higher accuracy relative to the workspace sizes when the data is normalized. Surprisingly, the accuracy obtained from the model of a bigger soft robot based on real-time FEM does not decrease proportionally to a bigger size. On the other hand, if the mean errors are relative to the full-length of the robot, they are around 5\% for both the heuristic and the MOR model. 


\subsection{Discussion}

It can be concluded that discrete hyper-redundant robots are relatively easy to model following the rigidbody mechanics whereas their kinematics can be accurately extracted by using the Denavit-Hartenberg convention.

On the other hand, continuous robots present some degree of compliance. In other words, their movements are nonlinear and usually harder to model. Thus, several techniques have been proposed to overcome this problem. However, the majority of strategies tend to accurately describe their movements through geometry shapes or dynamic models in absence of external objects. And continuous robots are especially designed to move in constrained environments or interact with objects with high dexterity. Thus, models that are not able to describe how a continuous robot behaves when contacting external objects can be considered as insufficient. This problem is even more exacerbated when modelling soft robots, since their main purpose is oriented towards safely interacting with their environment. Because of this, a hybrid method was developed as a combination of a modelling strategy with an on-board sensory system. The modelling strategy chosen to model the continuous robot was the Piecewise Constant Curvature or PCC, which provides high relative errors based in simple and efficient principles. And this errors were corrected in real time using the data from an IMU array system obtaining good performances overall.

However, the method that has more scientific support to become the most complete in order to model continuous and soft robots is the real-time Finite Element Method (FEM). This kind of practices are traditionally known to have high computational costs. However, several software optimizations and the exponential technological growth in informatics made this strategy feasible for real-time applications while retaining their well-known accuracy. Eventually, this method decomposes the robot in small elements that, adequately tuned, can extract the dynamic and material properties such as the mass, stiffness or slippery conditions, among many others. In addition, it is the only modelling technique that constitutes an integral solution to predict the robot movements considering both complex morphologies and also the surrounding elements and the environment.

Therefore, the soft Kyma was modelled following the real-time FEM. Likewise lots of soft devices, Kyma is composed by a thin body, usually difficult to model using FEM because of requiring a lot of elements, often unsuitable for real-time applications. This is an important issue that had to be addressed to define the deformation model. Two methods are presented to optimize the FEM efficiency and reduce the computational time. First, a heuristic method proposes to tune a model with fewer elements to match the behaviour of the real robot. Second, the Model Order Reduction technique may be the most promising one from the state-of-the-art. The heuristic method aims at solving this issue by slightly reducing the robot model accuracy in favour of experimentally adjusting other parameters to still capture the macroscopic deformations in real-time. On the other hand, the Model Order Reduction uses a set of offline simulations to reduce the number of state variables, retaining the main characteristics of the robot. In both scenarios, an actuation system based on tendons and a collision model have been developed to manage self and possible robot-object interactions.

Additionally, this work features a brief study of the compliance of the models, since soft robots are usually devised for that purpose. Consequently, the developed models exhibit high dexterity and good capabilities to surround or lift objects using the robot's body. It is also concluded that the relation between the characteristics of the robot and the properties of the objects is crucial to perform specific tasks. Thus, simulation results show that soft robots also have a lot of potential in space exploration activities where the action of gravity is negligible. 
To finish, both models have been validated using a very precise motion tracking system and making comparisons with the real robot. As a result, the control points were properly located with lower mean errors than in similar previous works. The MOR model presents a slightly higher accuracy than the heuristic one, and results obtained from the first one are more reliable than those from the second one since they are obtained without making any manual adjustment. Additionally, it can be deduced that this work studies the real-time FEM method for much bigger robots. And most importantly, the accuracy obtained from the model validation does not decrease proportionally to a higher robot size. Moreover, both models exhibit accuracies up to 3 times higher than previous methods such as PCC. However, future works should not lose sight of other approaches like two-dimensional shell elements since they may also provide low computational costs for thin morphologies [249].

With these results, it is possible to discuss whether a the real-time FEM model can be valid or not. In the context of this work, the obtained models manage to capture the main macroscopic shapes of a very complex device, opening the way for a promising future to predict the movements of soft robots and take reliable control actions. 


\section{Control}

This section presents a novel strategy to apply a high-level control to hyper-redundant, continuous and soft robots. In other words, to manage the movements of their whole kinematic chain. Thus, a new method to solve the inverse kinematics problem is firstly presented. From an engineering perspective, this kind of robots are underdetermined systems. Therefore, they exhibit an infinite number of solutions for the inverse kinematics problem, and to choose the best one can be a great challenge. A new algorithm based on the Cyclic Coordinate Descent (CCD) and named as Natural-CCD is proposed to solve this issue. It takes its name as a result of generating very harmonious robot movements and trajectories that also appear in nature, like the Golden spiral. In addition, it has been applied to perform continuous trajectories, to develop whole-body movements, to analyse motion planning in complex environments and to study fault-tolerance, even for both prismatic and rotational joints. The proposed algorithm is very simple, precise and computationally efficient. It works for robots either in two or three spatial dimensions and handles a large amount of degrees-of-freedom. Additionally, it was found that modifying and reinterpreting the three basic variables needed to solve the inverse kinematics problem, a complete new paradigm emerged. This modifications lead to a number of novel, decentralized control behaviours. These primal behaviours were not designed but rather emerged as a result of distributed, incremental and uniform movements in the robot body using the Natural-CCD algorithm. They include different modes of attraction, repulsion, surrounding, straightening, folding and circumnutations. Opposed to traditional control approaches which are typically programmed for one specific application, this framework provides a set of general behaviours that can combined for different scenarios.

Further information can be found in original previous publications ${ }^{14,15}$. Also, an explanatory of this video can be found in the Electronic Supplementary Material (Videos S. 3 and S. 4).

\footnotetext{
${ }_{14}$ Andrés Martín-Barrio et. al."The Natural-CCD algorithm: A novel method to control hyper-redundant and soft robots." Soft Robotics 5, no. 3 (2018) 242-257.

${ }^{15}$ Andrés Martín-Barrio et al., Design of a Hyper-Redundant Robot and Teleoperation Using Mixed Reality for Inspection Tasks. Sensors 20.8, 2020: p. 2181.
} 


\subsection{The Natural-CCD algorithm}

Methods like the Cyclic Coordinate Descent (CCD) proposes one solution for this problem. It is a derivativefree optimization algorithm that finds the minimum of a multi-variable function by uniformly and cyclically minimizing each variable. [250] proposed an approach based on $C C D$ to solve the inverse kinematics problem. Let a kinematic chain be defined by a discrete number of control points that can be connected to define a robot shape. Thus, a single control point can contain one or more degrees-of-freedom. Then, $\mathbf{p}_{\mathbf{c}}$ can be defined as a variable that takes the position of every control point $(i)$ cyclically from $i=[1, n], \mathbf{p}_{\mathbf{e}}$ the end-effector position, and $\mathbf{p}_{\mathbf{f}}$ the desired end-effector position. The position of the control points can be defined as $P\left(\mathbf{q}_{\mathbf{i}}\right)$ where $\mathbf{q}_{\mathbf{i}}=\left\{q_{1}, q_{2}, \ldots, q_{i}\right\}$. is the joint space (Eq. 5.1).

$$
\begin{aligned}
& \boldsymbol{p}_{c}=P\left(\boldsymbol{q}_{\boldsymbol{i}}\right) \\
& \boldsymbol{p}_{e}=P\left(\boldsymbol{q}_{\boldsymbol{n}}\right) \\
& \boldsymbol{p}_{f}=\boldsymbol{Q} \in I R^{m}
\end{aligned}
$$

Then, it is possible to find an angle $\theta_{i}$ (Eq. 5.2) and a direction $\mathbf{d}_{\mathbf{i}}$ (Eq. 5.3) to rotate every control point and minimize the distance $\left\|\mathbf{Q}-P\left(\mathbf{q}_{\mathbf{n}}\right)\right\|$ as seen in Figure 5.1.

$$
\begin{gathered}
\theta_{i}=\cos ^{-1}\left(\frac{\boldsymbol{p}_{e}-\boldsymbol{p}_{\boldsymbol{c}}}{\left\|\boldsymbol{p}_{e}-\boldsymbol{p}_{c}\right\|} \cdot \frac{\boldsymbol{p}_{f}-\boldsymbol{p}_{\boldsymbol{c}}}{\left\|\boldsymbol{p}_{f}-\boldsymbol{p}_{c}\right\|}\right) \\
\boldsymbol{d}_{\boldsymbol{i}}=\frac{\boldsymbol{p}_{\boldsymbol{e}}-\boldsymbol{p}_{\boldsymbol{c}}}{\left\|\boldsymbol{p}_{e}-\boldsymbol{p}_{\boldsymbol{c}}\right\|} \times \frac{\boldsymbol{p}_{f}-\boldsymbol{p}_{\boldsymbol{c}}}{\left\|\boldsymbol{p}_{f}-\boldsymbol{p}_{c}\right\|}
\end{gathered}
$$

$\mathbf{a}$

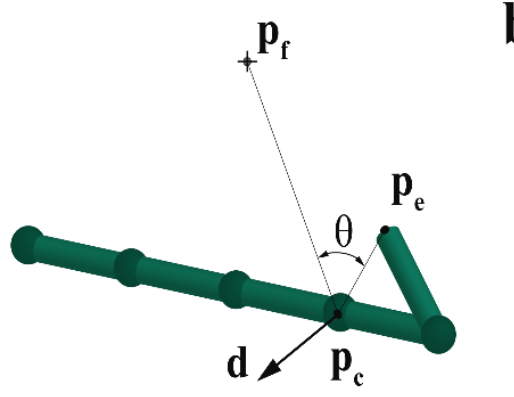

b

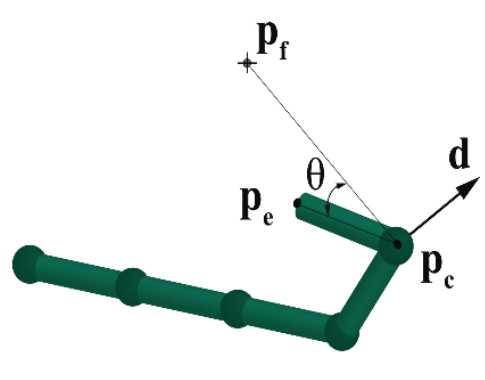

Figure 5.1 The Cyclic Coordinate Descent algorithm rotates every control point so the distance between the end-effector and the desired target is minimized [11].

This method is very simple and computationally efficient for hyper-redundant robots. However, it has several limitations. It does not manage singularities or self-collisions, and high values of $\theta_{i}$ usually lead to complex solutions. Other works [251], [252] proposed useful modifications to overcome these problems.

However, [11] introduced a different alternative that provides a set of intermediate configurations for the inverse kinematics: the Natural-CCD algorithm. It is based on the idea of limiting the value of $\theta_{i}$ to small increments using a maximum angle $\theta_{\max }$ (Eq. 5.4), so the robot iteratively and smoothly converges to solutions in real-time even for thousands of degrees of freedom [11].

$$
\theta_{i}^{*}=\max \left(\theta_{i}, \theta_{\max }\right)
$$

The Natural-CCD method is based on incremental, cyclical and uniform movements. Additionally, the Natural-CCD algorithm manages kinematic singularities. When vectors $\overrightarrow{p_{c} p_{f}}$ and $\overrightarrow{p_{c} p_{e}}$ are parallel, the 
mentioned $\vec{d}$ direction results in a null vector (Eq. 5.3). In this case, it is possible to decide not to move the corresponding joint, because the movement of the previous ones may help avoiding that singularity. However, there are some situations in which all the joints are blocked by singularities; for example, when all of them are collinear with the desired position. In those special cases, the use of a random direction $\vec{d}$ (Eq. 5.3) and angle $\theta$ (Eq. 5.2) is recommended to avoid the initial singularity.

Moreover, this method manages the existence of self-collisions. For this purpose, the distance and direction of movement of each section is compared to the rest of sections while also considering the girth of the robot. Interestingly, if the kinematic chain is inverted, $p_{f}$ will be taken as the base of the robot and the application of the algorithm will lead to an Endpoint control. With that scheme, the base of the robot can move such as in a mobile platform while the tip of the robot remains in a given location. This could be very interesting to perform multiple tasks in with high dynamics involved.

The main structure of the Natural-CCD algorithm can be found in Table 5.1. Some authors have proposed other Coordinate Descent approaches, such as the Adaptive Coordinate Descent (ACiD) [253], which uses adaptive encoding to gradually build a transformation of the coordinate system. With this method, the new coordinates are as decorrelated as possible with respect to the objective function. Another technique worth mentioning is the Randomized Coordinate Descent [254], which selects randomly with uniform probability the coordinate to minimize.

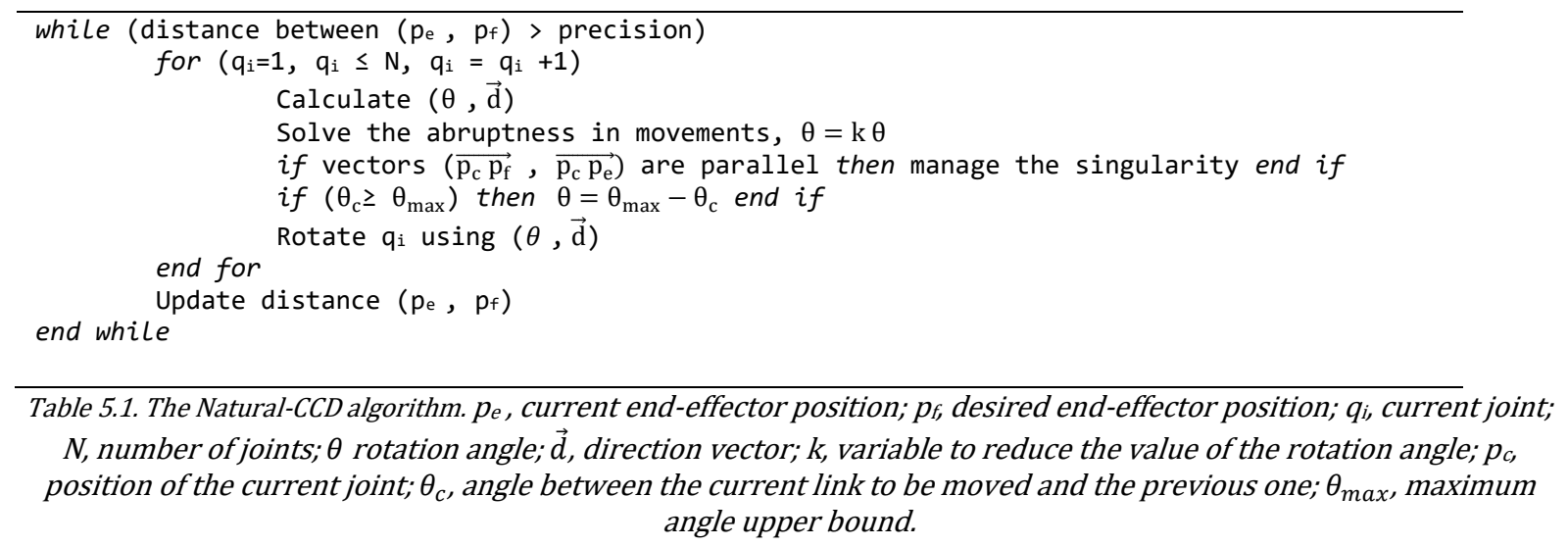

In this work a novel alternative called Selective Coordinate Descent (SCD) is also presented. The aim is to use this new approach in underdetermined systems like hyper-redundant robots. The main difference between CCD and SCD is that the first one minimizes all the coordinates or variables uniformly, whereas the second one does not. The SCD selects a candidate to be moved among all the elements so as to meet a specific requirement. For example, in the inverse kinematics problem of hyper-redundant robots, the joint to be moved, in terms of minimizing energy, could be chosen in each cycle. This can be also combined with the modifications presented in the Natural-CCD algorithm as shown in Table 5.2. Also, a comparison between both techniques is shown in Figure 5.2. 


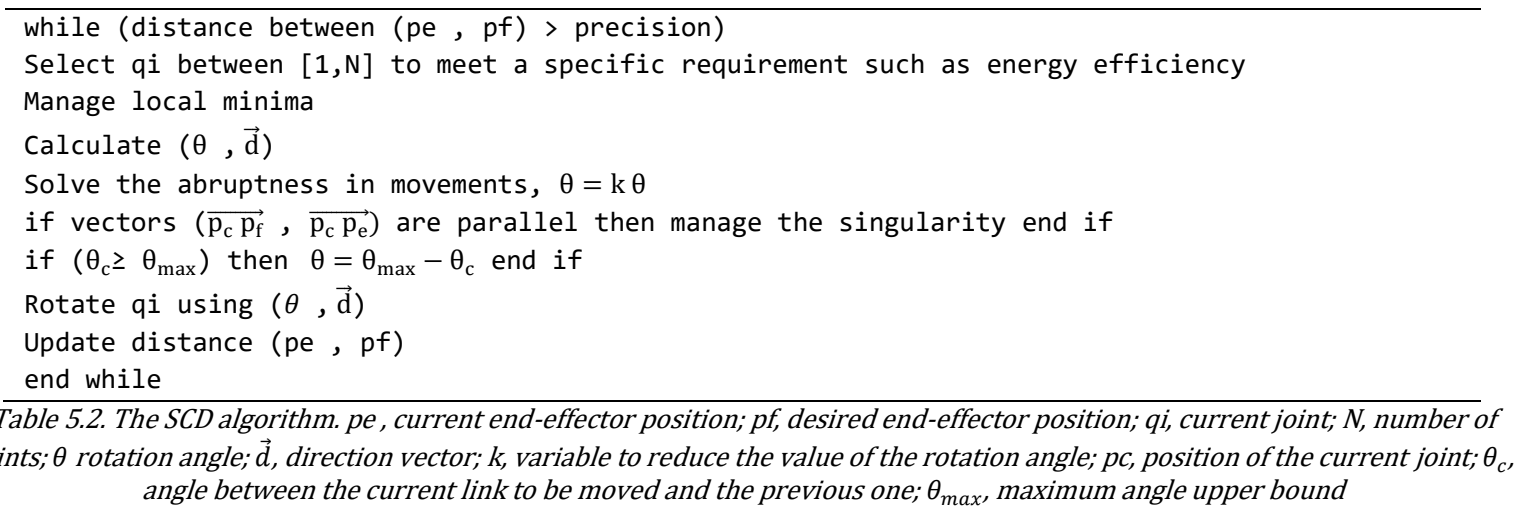

a

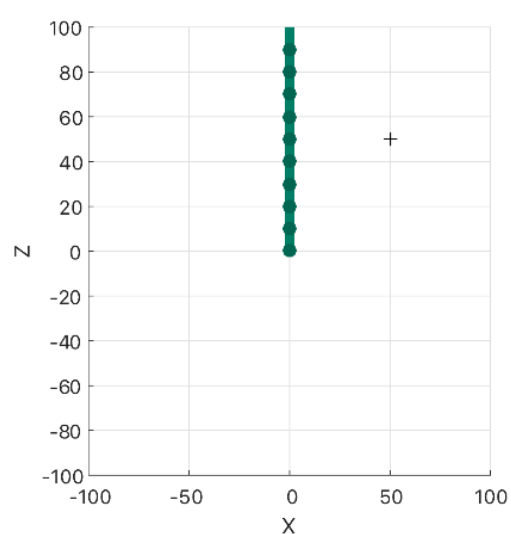

b

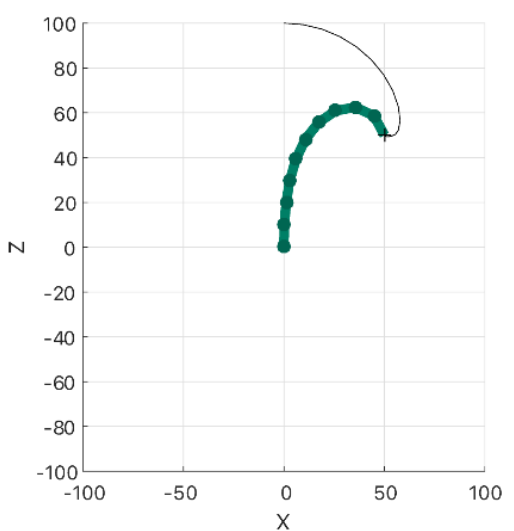

C

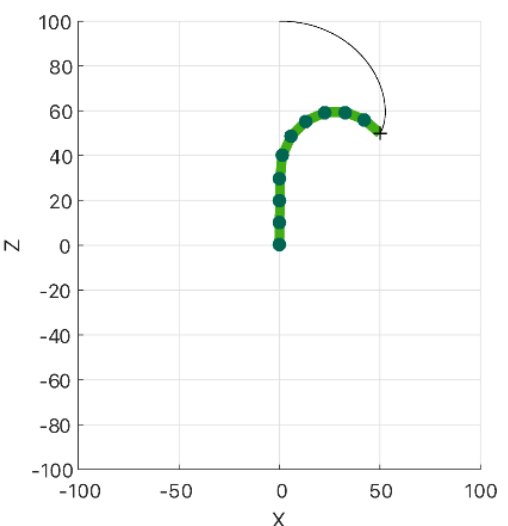

Figure 5.2. Movement of a hyper-redundant robot with 10 rotational joints (a) from a straight configuration to a desired position, (b) using the Natural-CCD algorithm (c) and the Natural-SCD algorithm.

Moreover, this method can also take advantage of prismatic actuators or growing mechanisms to reduce the distance $\left\|\mathbf{p}_{\mathbf{e}}-\mathbf{p}_{\mathbf{f}}\right\|$, as shown in Eq. 5.5, where $L_{i}$ is the length between two joints and $\Delta L$ a positive or negative increment.

$$
L_{i}=\left\|P\left(\boldsymbol{q}_{i+1}\right)-P\left(\boldsymbol{q}_{i}\right)\right\|+\Delta L
$$

This prismatic or growing capabilities can be limited by a maximum and a minimum restriction to simulate real robot behaviours. When the length between two joints reaches the maximum elongation, it is theoretically possible to add a new joint in between, such as in the middle, letting the robot to grow (Figure 5.3). 
a

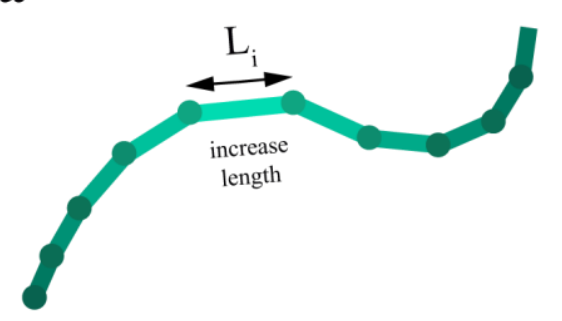

b new control

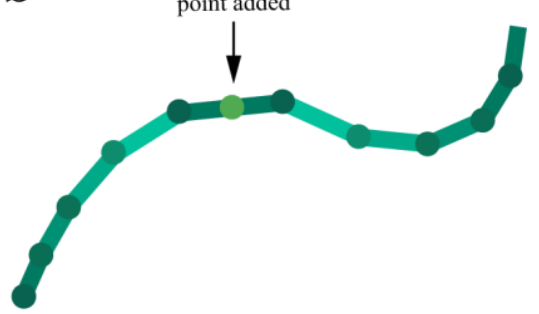

Length

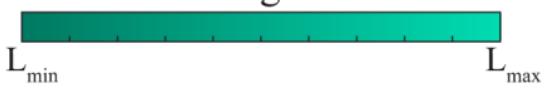

Figure 5.3. Kinematic growing process from a discrete perspective. (a) When a module extends its length $L_{i}$ close to a predefined maximum constraint, (b) a new control point is added to the configuration, increasing the robot maneuverability.

\subsubsection{Biomimetics}

It can be said that some trajectories resulting from the Natural-CCD algorithm are biomimetic or similar to those observed in nature. Biomimicry derives from the Ancient Greek: bios (life) and mimesis (imitation). It is the science that studies nature as a source of inspiration and new innovative technologies to solve human problems, which the nature itself has already solved through system models, processes and elements that are inspired therein [255].

The Figure 5.4 shows the movement of a two-dimensional robot with ten rotational joints and ten equallength links. It starts from an upright configuration, with a desired end-effector position close to the anchored base and with no orientation restrictions. It can be seen that the final configuration is close to a decagon. In this particular case, it is result of the incremental and uniform movement of all the joints, as it will be explained.

a

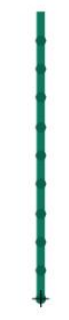

b

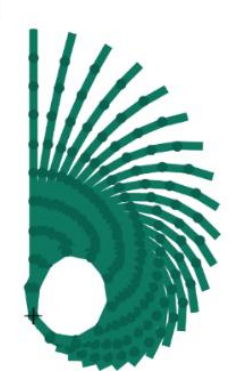

C

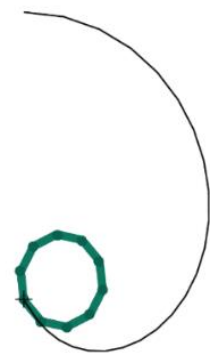

Figure 5.4. Movement of a planar robot with 10 rotational joints using the Natural-CCD algorithm. (a) Initial straight configuration. (b) Intermediate configurations. (c) Final configuration and the end-effector path representation.

The final end-effector path has been interpolated one time per cycle, only when all joints have had the chance to be moved, as other authors have done in previous works [250]. In this case, the resulting paths within a two-dimensional space are often quite particular. They tend to shapes commonly present in nature: Logarithmic spirals.

A logarithmic spiral, equiangular spiral or growth spiral is a self-similar spiral curve for which the radius grows exponentially with the angle (Figure 5.5). It has the following mathematical definition in Cartesian coordinates (Eq. 5.6) and in Polar coordinates (Eq. 5.7), where $r$ is the distance from the origin, $\alpha$ is the angle from the x-axis and $a$ and $b$ are arbitrary constants. 


$$
\begin{aligned}
& x=r \cos \alpha=a e^{\mathrm{b} \alpha} \cos \alpha \\
& y=r \sin \alpha=a e^{\mathrm{b} \alpha} \sin \alpha
\end{aligned}
$$

$$
r=a e^{\mathrm{b} \alpha}
$$

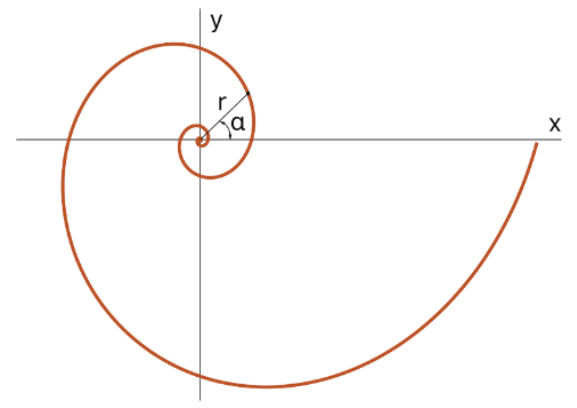

Figure 5.5. Representation of a logarithmic spiral

In differential geometric terms, the logarithmic spiral can also be defined with the constant angle $\beta$ (Eq. 5.8), measured from the tangent and radial vectors at every single point of the curve $(r, \alpha)$. The derivative of $r(\alpha)$ is proportional to the $b$ parameter. In other words, it determines how closely and in which direction the spiral wraps. In the extreme case, where $b$ is equal to zero, the spiral would be a circle of radius $a$, and if it approached to infinity, the spiral would tend to be a ray.

$$
\beta=\cos ^{-1} \frac{\left\langle r(\alpha), r^{\prime}(\alpha)\right\rangle}{\|r(\alpha)\|\left\|r^{\prime}(\alpha)\right\|}=\tan ^{-1} \frac{1}{b}
$$

When the robot has an initial upright configuration, all of its structure temporally belongs to a unidimensional space. On that basis, when the robot is moved using the Natural-CCD algorithm and with no orientation restrictions, the joints will belong to a plane. In this scenario, some of the resulting end-effector paths tend to be logarithmic spirals. This can be tested using the Cesàro equation. In geometry of plane curves, this equation provides an indicator of the curvature $\kappa$, which is the reciprocal of the radius. It can be said that two curves are congruent if they have the same Cesàro equation. In geometry, two figures or objects are congruent if they have the same shape and size, or if one has the same shape and size as the mirror image of the other. The Cesàro equations of some types of curves are the ones as follows (Eq. 5.9-Eq. 5.12), where $R$ is the radius, $C$ is a constant and $s$ is the arc length [256]:

- Straight line: $\mathrm{\kappa}=0$

- Circle: $\mathrm{K}=\frac{1}{R}$

- Circular involute: $\mathrm{\kappa}=\frac{C}{\sqrt{s}}$

- Logarithmic spiral: $\mathrm{\kappa}=\frac{C}{s}$

If the curve produced by the end-effector is parameterized, it is possible to represent the relation between the curvature $\kappa$, with the arc length $s$, as it is shown in the Figure 5.6. For example, the particular movement of the previous Figure 5.4, tends to a logarithmic spiral since the arc length and the curvature are inversely proportional (Eq. 5.12). 


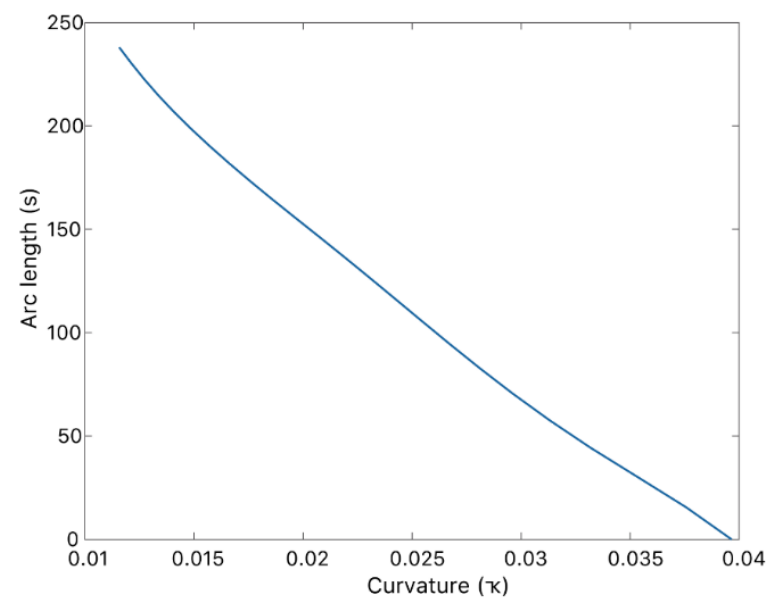

Figure 5.6. Curvature-arc length test results for the movement of a robot with 10 links using the Natural-CCD algorithm from a straight configuration and with a desired end-effector position closed to the anchored base.

Moreover, it has been found that this particular path is, in fact, a specific type of logarithmic spiral: a golden spiral. This spiral is characterized by having a growth factor equal to $\varphi$, the golden ratio or divine proportion. In mathematics, two quantities are in the golden ratio if their proportion is the same as the ratio of their sum to the larger of the two quantities (Eq. 5.13-Eq. 5.14).

$$
\frac{u+v}{u}=\frac{u}{v} \text { with } u>v
$$

$$
\begin{gathered}
\varphi=\frac{u}{v} \\
1+\varphi^{-1}=\varphi \\
\varphi^{2}-\varphi-1=0 \\
\varphi=1.61803398 \ldots
\end{gathered}
$$

A widely known approximation of the golden spiral is the Fibonacci spiral (Figure 5.7). This curve has numerous applications in number theory, applied mathematics, computer science, physics and biology [257]. Its popularity comes from its appearance in numerous figures of nature such as branches of trees, arrangement of leaves on the stem, flowers of artichokes and sunflowers [258], inflorescences of Romanesco broccoli, horns and claws of some mammals, phalanges of human hands, sea waves, tails of comets or even arms of galaxies. The Fibonacci spiral is generated drawing circular arcs that connect the opposite corners of several squares beside each other, having as sides the values of the Fibonacci sequence (Eq. 5.15).

$$
x(k+2)=x(k+1)+x(k)
$$




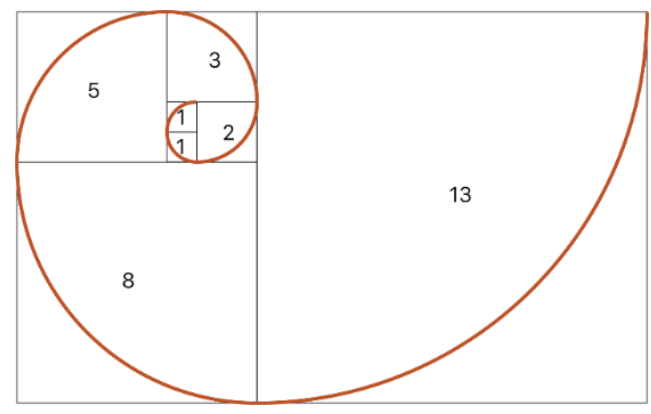

Figure 5.7. The Fibonacci spiral constructed from squares with same size as the Fibonacci sequence.

Owing to this concept, the Golden spiral and consequently its approximation, the Fibonacci spiral, can be defined according to the Eq. 5.8 (Eq. 5.16-Eq. 5.18).

$$
\begin{gathered}
e^{b_{f} \alpha_{\text {right }}}=\varphi \\
\left|b_{f}\right|=\frac{\ln \varphi}{\pi / 2}=0.306349 \mathrm{rad} \\
\beta_{f}=\tan ^{-1} \frac{1}{b_{f}}=72.9676^{\circ}
\end{gathered}
$$

It can be demonstrated that the resulting path from Figure 5.4 is a Fibonacci spiral by polynomial curve fitting. It has been found that this appears for a sufficiently redundant number of equal-length links according to the Eq. 5.18.

It can be seen in Figure 5.8 that for a number higher than 10 links, the resulting path tends to be a Golden spiral (Figure 5.9). There exists a slightly difference between the stabilization of the theoretical constant angle $\beta_{f}$ and the obtained from the resulting path $\beta$, because the robot is discretized in a finite number of serial links, instead of being infinitely bendable on all of its points. Due to the application of Eq. 5.4, all the joints are rotating nearly the same small amount in each cycle, converging to the desired position. Because of this, from a kinematics perspective, it is possible to set forth the following statement.

A serial manipulator robot with a sufficiently redundant number of equal-length links can determine a biomimetic end-effector path, or Fibonacci spiral, by using the Natural-CCD algorithm, when their rotational joints are cyclically and uniformly moved from an upright configuration bit by bit to a position close to its base. 


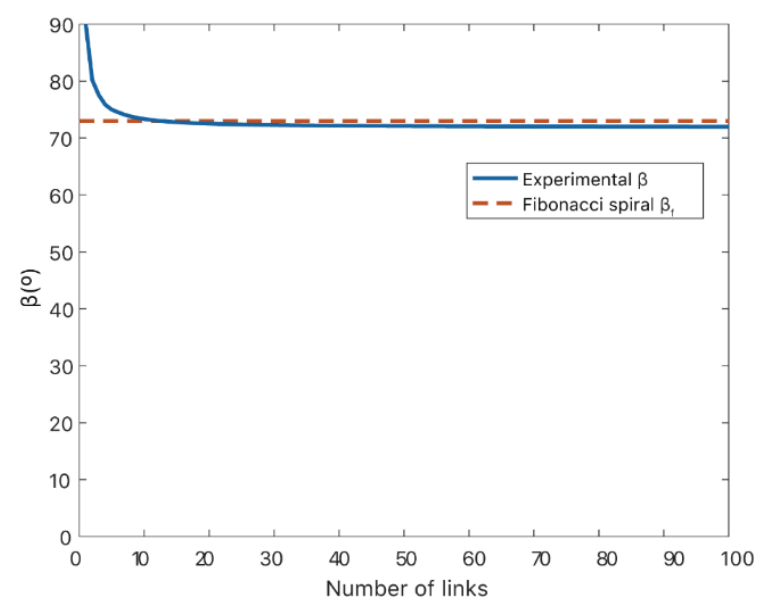

Figure 5.8. Comparison of the Fibonacci spiral constant angle $\beta_{f}$ and the obtained angle $\beta$ from the resulting path presented in Figure 5.4 versus the number of links of the robot.

a

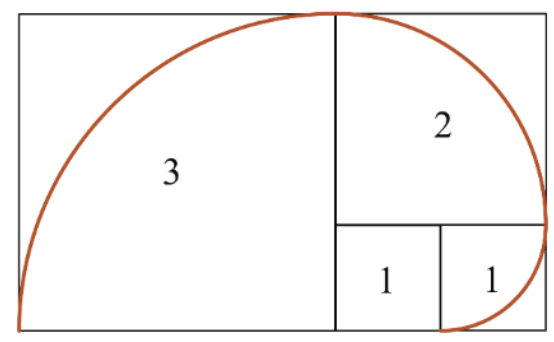

b

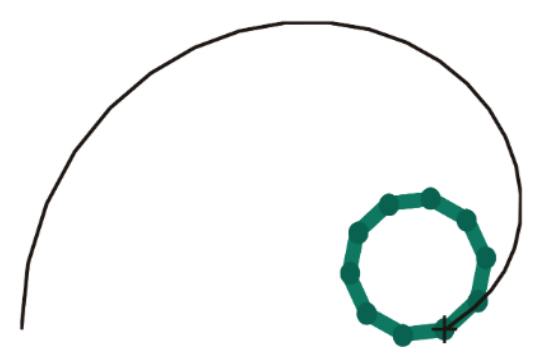

Figure 5.9. Comparison of (a) the Fibonacci spiral and (b) the resulting end-effector path using the Natural-CCD algorithm.

The demonstration of the biomimetic movement of the robot in Figure 5.4 gives reason to think that the harmonious movements originated by the Natural-CCD algorithm are, to some extent, biomimetic or related to nature. In order to generalize that theory, the proposal is to imagine a robot with an infinite number of links. In fact, this could be a soft robot since it is continuous. In that context, the same statement can be expressed in geometric terms as follows.

If a straight segment with a fixed length and anchored on one end is cyclically, uniformly and infinitesimally bended on all of its points along the same direction, the other end will move along a Golden spiral until enclosing the initial straight segment to a circle.

It is expected that this property can help to clarify the common appearance of the Fibonacci spiral in numerous figures of nature. After all, it is related to the behaviour of a very simple and familiar movement. Another way to visualize this phenomenon is by approximating the robot configurations to arcs of circumferences. Initially, since the configuration is a straight segment, it belongs to a circumference with an infinite radius. The length of the robot remains invariable with the movement, but it does vary the radius and the central angle of the circumferences that contain the robot on each cycle (Figure 5.10). 


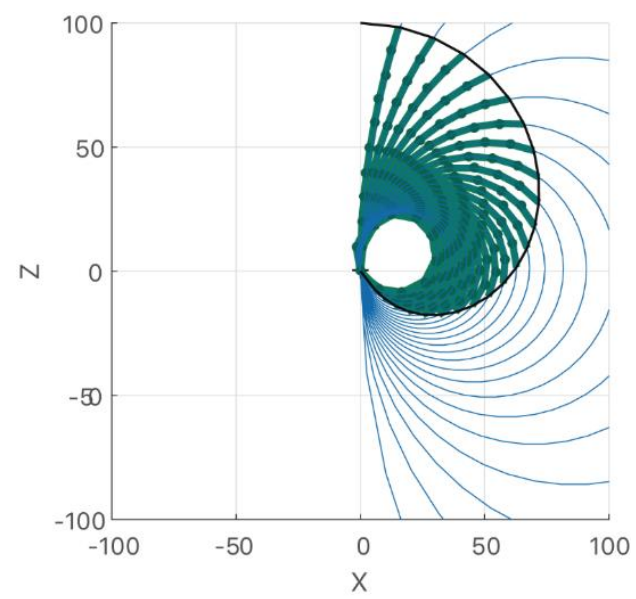

Figure 5.10. The Fibonacci spiral path and representation of the circumferences around the robot configurations.

\subsubsection{Features}

A unified, simple and clean interface was used to customize the hyper-redundant robot as desired (Figure 5.11). It includes multiple features that will be further analysed throughout this chapter.

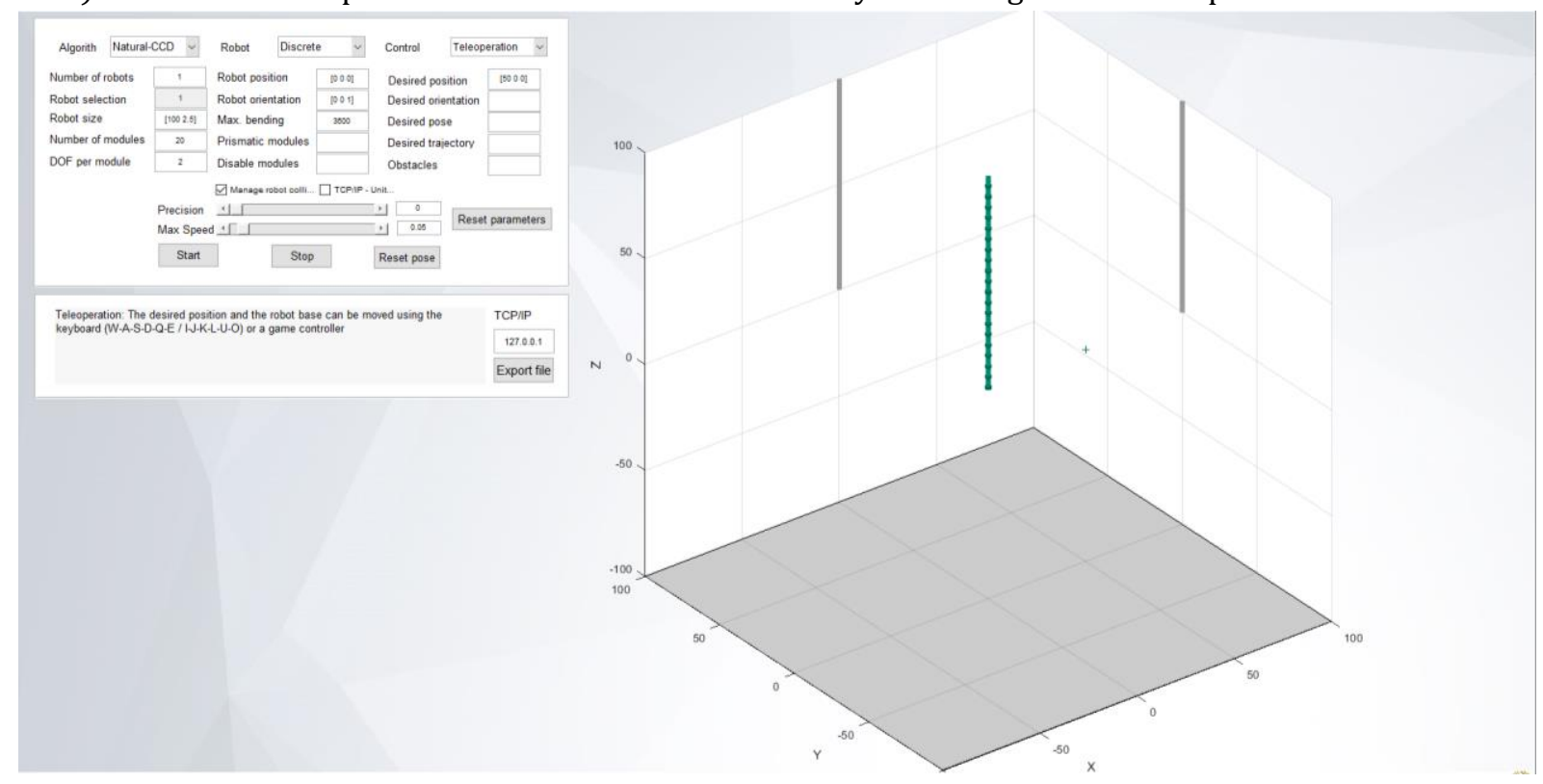

Figure 5.11. Interface created in MATLAB to customize and supervise the control of the hyper-redundant robot.

The CCD algorithm provided a desired position and orientation error minimization for either rotational or prismatic joints [250]. This has been tested using the Natural-CCD as well. Figure 5.12 shows the movement of a robot for a desired position using the Natural-CCD algorithm and the variation of some important parameters. 
a

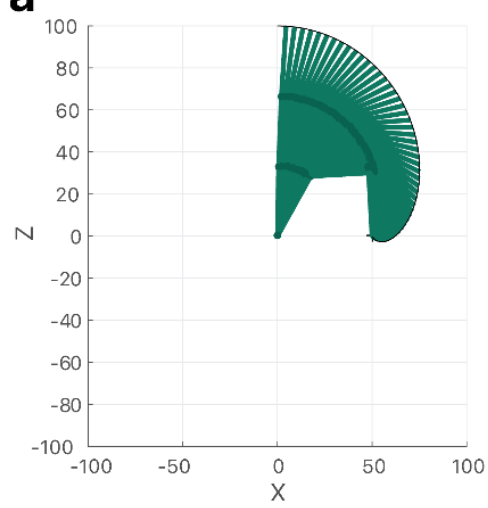

b

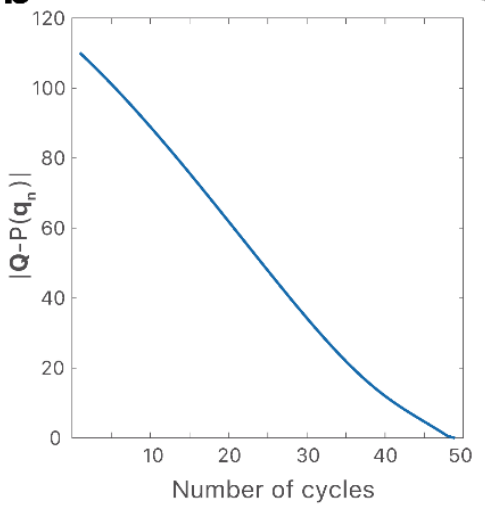

C

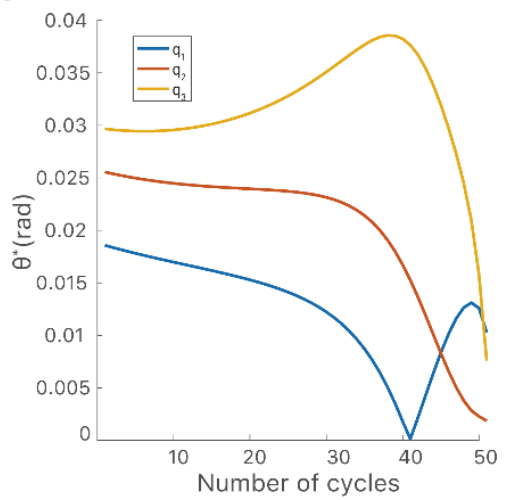

Figure 5.12. Movement of a robot with three rotational joints using the Natural-CCD from a straight configuration to a given desired point, where $q_{1}, q_{2}, \ldots, q_{n}$ are the robot joints from the base to the end-effector. (a) Representation of the intermediate configurations.

(b) Convergence of the end-effector to the desired position measured as a Euclidean distance. (c) Angle variation for each joint.

For a given position and orientation, the Natural-CCD algorithm stands out for providing very harmonious and coherent solutions (Figure 5.13).

a

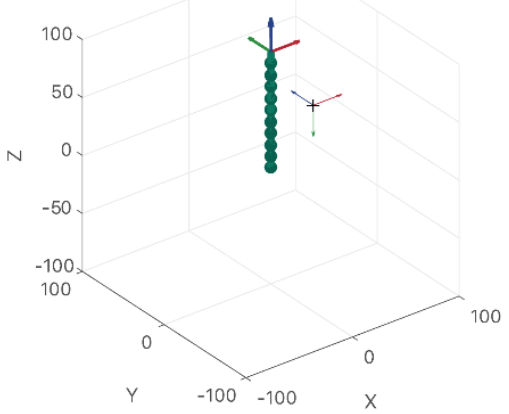

b

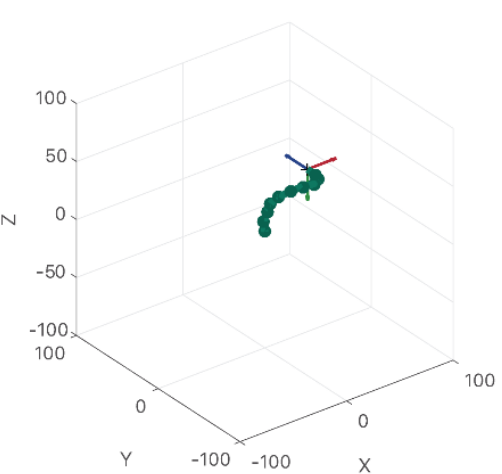

C

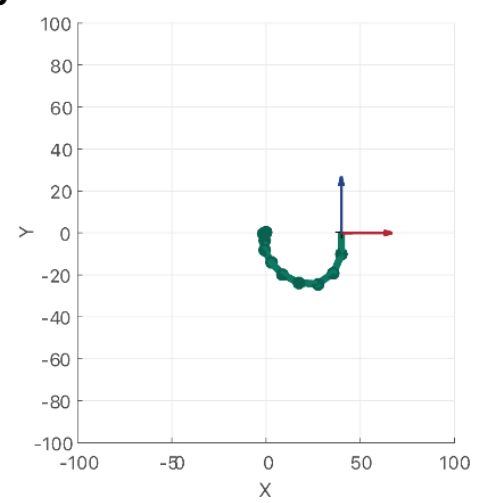

Figure 5.13. Movement of a hyper-redundant robot with 10 rotational joints and 3 DOFs per joint using the Natural-CCD. (a) Initial configuration and desired position and orientation for the end-effector. (b) Final configuration in a 3D perspective. (c) Top view of the final configuration.

Additionally, the combined use of rotational and prismatic joints have also been studied as Figure 5.14 depicts.

a

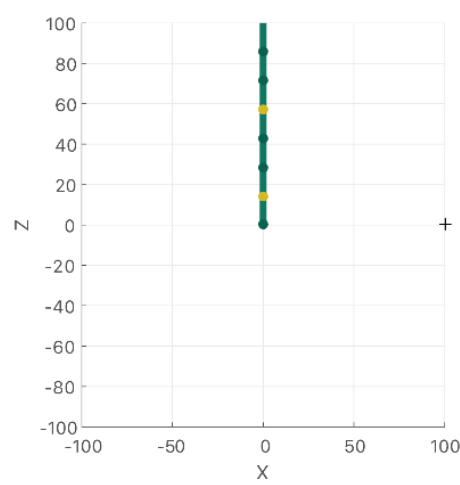

b

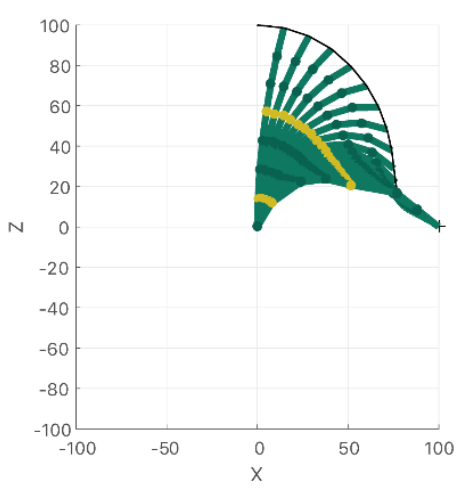

C

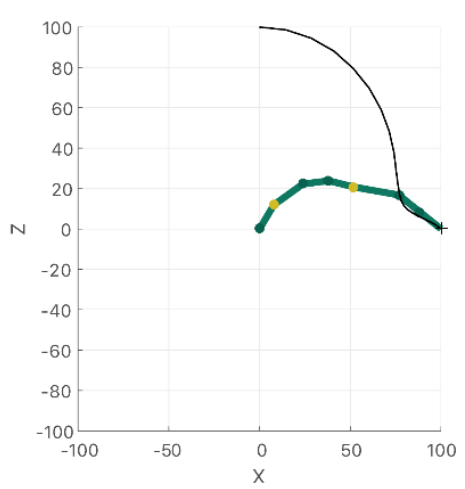

Figure 5.14. Movement of a robot with 5 rotational joints and 2 rotational and prismatic joints (yellow) from a straight configuration to a desired position using the Natural-CCD. (a) Initial straight configuration. (b) Intermediate configurations. (c) Final configuration. 
In addition to this, other important features have originally been studied in this work. For example, it is possible to disable the movement of a given joint in real-time to simulate a mechanical failure and exploit the fault-tolerance capabilities since it does not matter in what order the coordinates are minimized using the Natural-CCD (Figure 5.15).

a

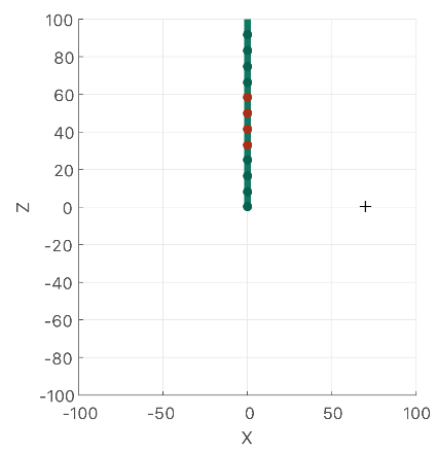

b

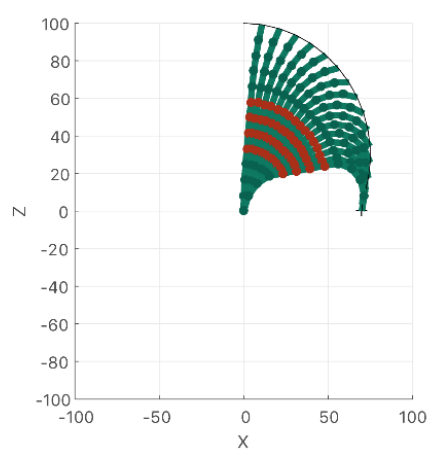

c

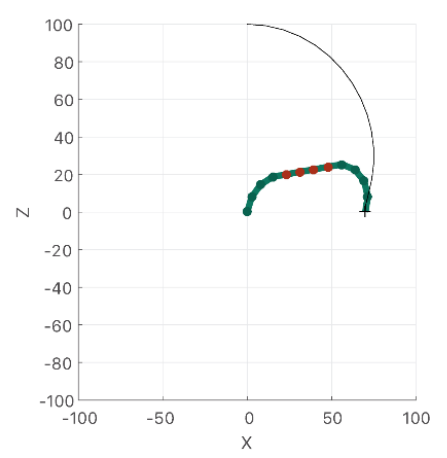

Figure 5.15. Simulation of failure of 4 joints (red) over a total of 12 rotational joints to study fault tolerance for hyper-redundant robots- (a) Initial straight configuration. (b) Intermediate configurations. (c) Final configuration.

Furthermore, the inverse kinematics algorithm can be applied for a continuous trajectory. This trajectory can be discretized as a set of desired positions that have to be reached sequentially under a certain precision, instead of having a single desired position (Figure 5.16). In this context, it is recommended to use the SCD algorithm instead of using the Natural-CCD. With that strategy, the joint with more manipulability to move along a particular trajectory can be selected in each iteration, allowing to significantly reduce the number of iterations.
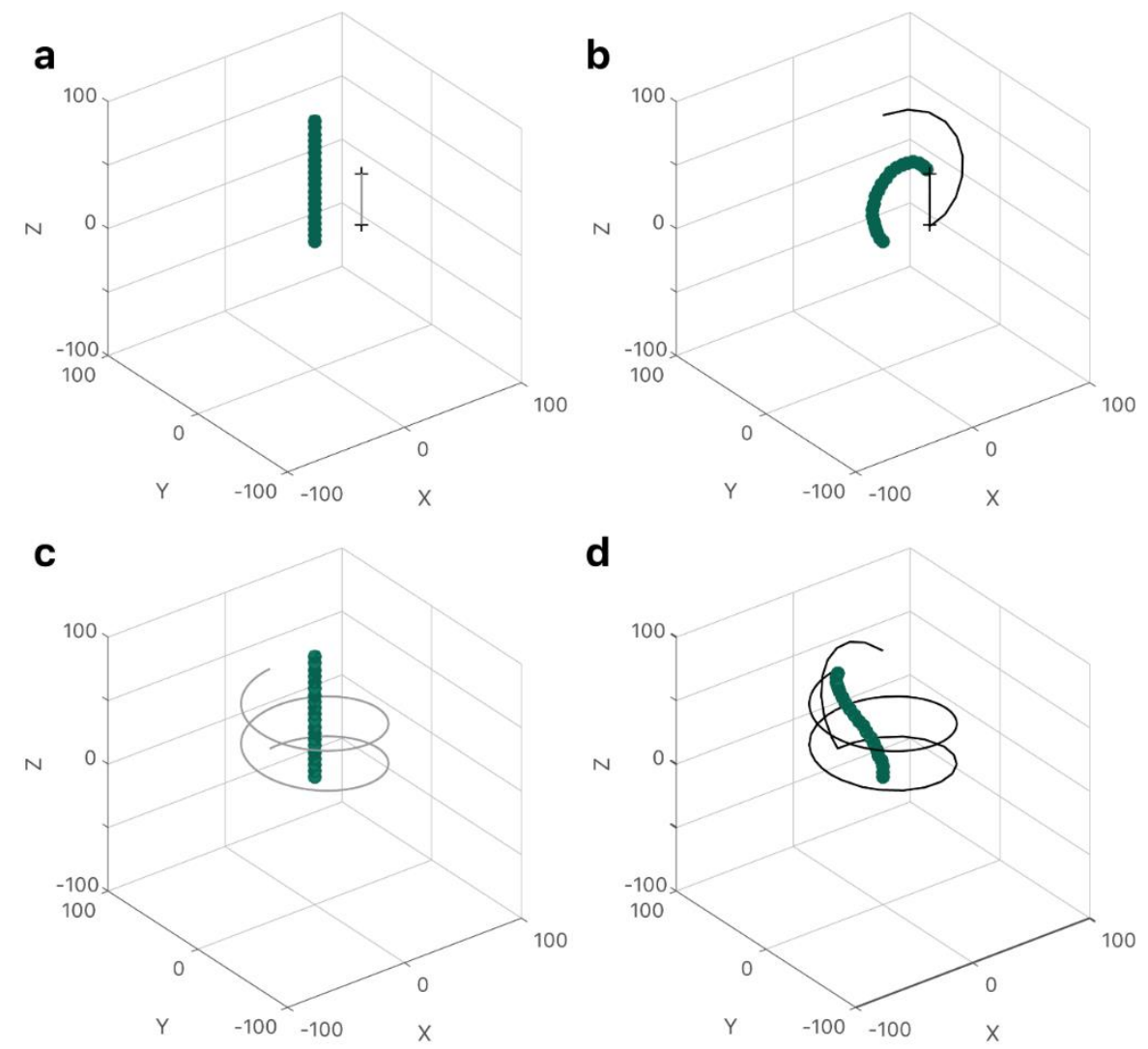

Figure 5.16. Trajectory planning of a robot with 20 rotational joints and 3 DOFs per joint (60 DOFS) using the SCD. (a) Desired straight-line trajectory. (b) Final configuration and end-effector path for the line trajectory. (c) Desired helical trajectory. (d) Final configuration and end-effector path for the helical trajectory. 
Moreover, a study of the obstacle-avoidance capabilities of hyper-redundant robots using the Natural-CCD has been made. The Natural-CCD algorithm lets the end-effector of the robot be attracted by the desired location. This can be combined with a robotic motion planning method, such as potential fields [259], in order to repel the obstacles in each iteration (Figure 5.17). All the obstacles have been virtually swollen a slightly higher amount than the half of the girth of the manipulator in order to prevent collisions. The main drawback of this strategy is that the algorithm can fall into a local minimum while minimizing the coordinates. To solve this issue, a tangential direction is determined in order to move the robot contouring the obstacle. This strategy can remind to a Bug Algorithm [260], a complete path planning method for mobile robots.

a

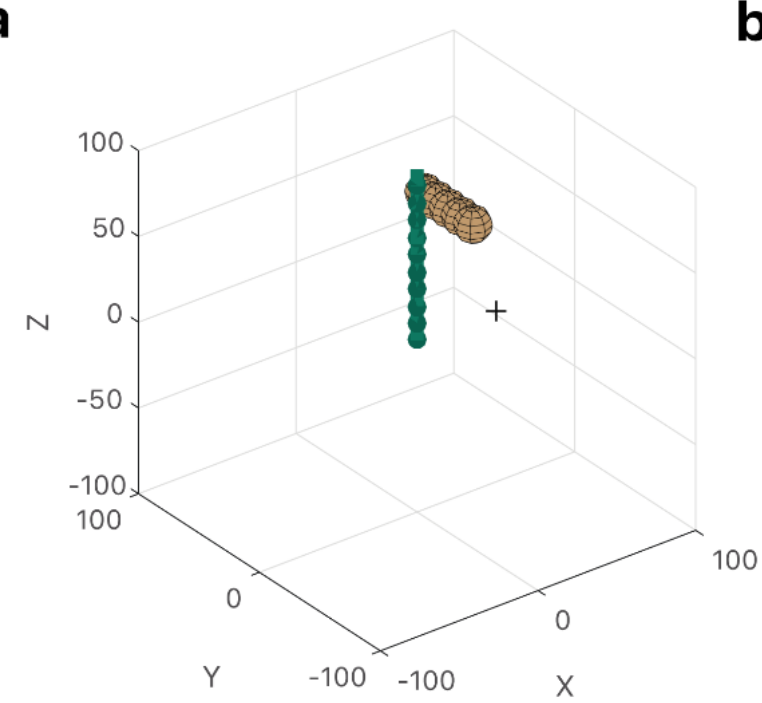

b

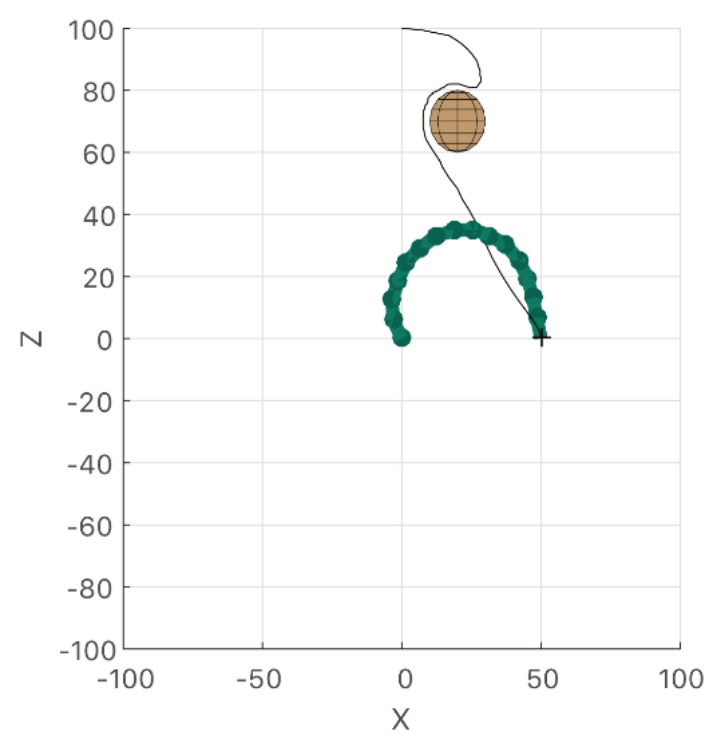

Figure 5.17. Combined use of Natural-CCD and potential fields to avoid the obstacles modelled as consecutive spheres. (a) Initial robot configuration, obstacles and desired position. (b) Final configuration and path.

A collision-free trajectory from the initial configuration to the desired position has been calculated when the obstacles and the environment are more complex, such as introducing the robot through a hole in a wall (Figure 5.18). Thus, this trajectory will guide the end-effector while the rest of the robot is repelled from the obstacles if necessary. This trajectory cannot be any collision-free trajectory since the end-effector is not a point mobile robot and it is linked to the joints towards its base. This approach avoids the use of the offline calculation of the Configuration Space or C-Space [73], which results in a high computational cost for hyper-redundant manipulators. 

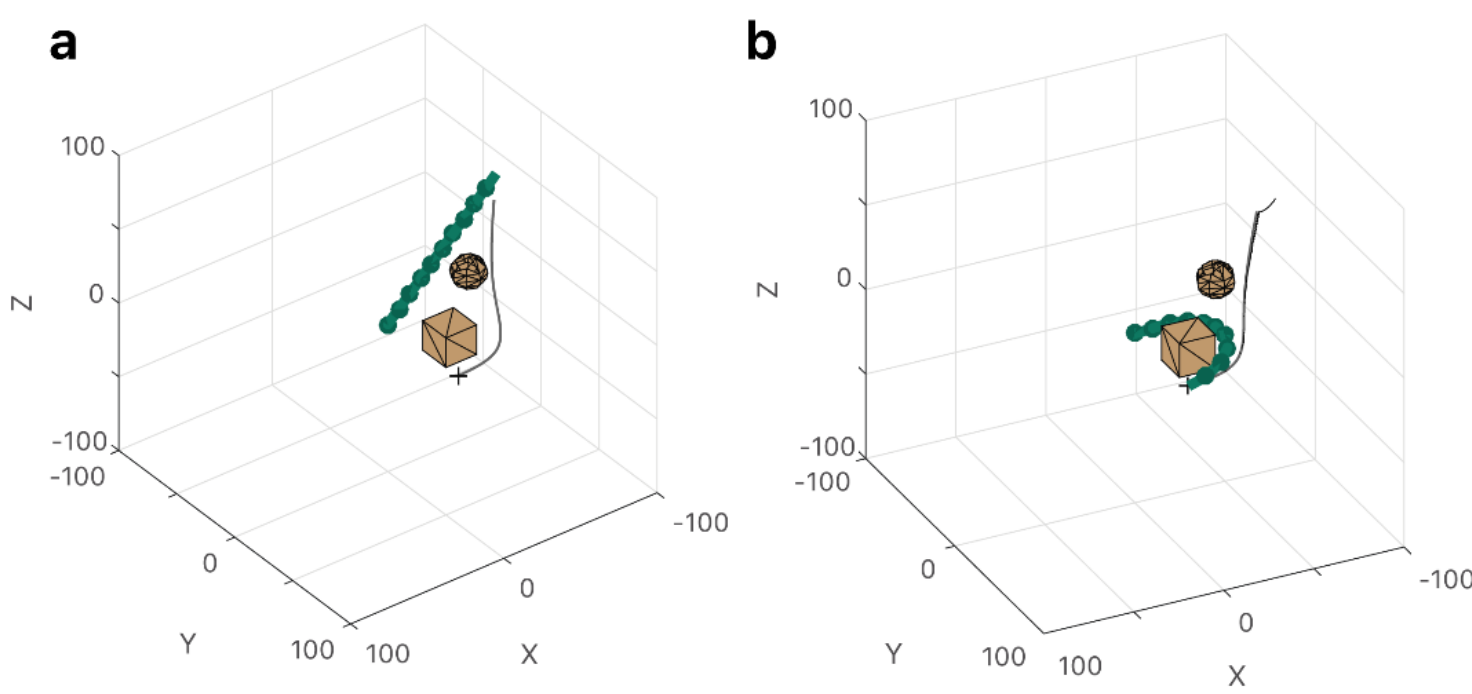

C

d
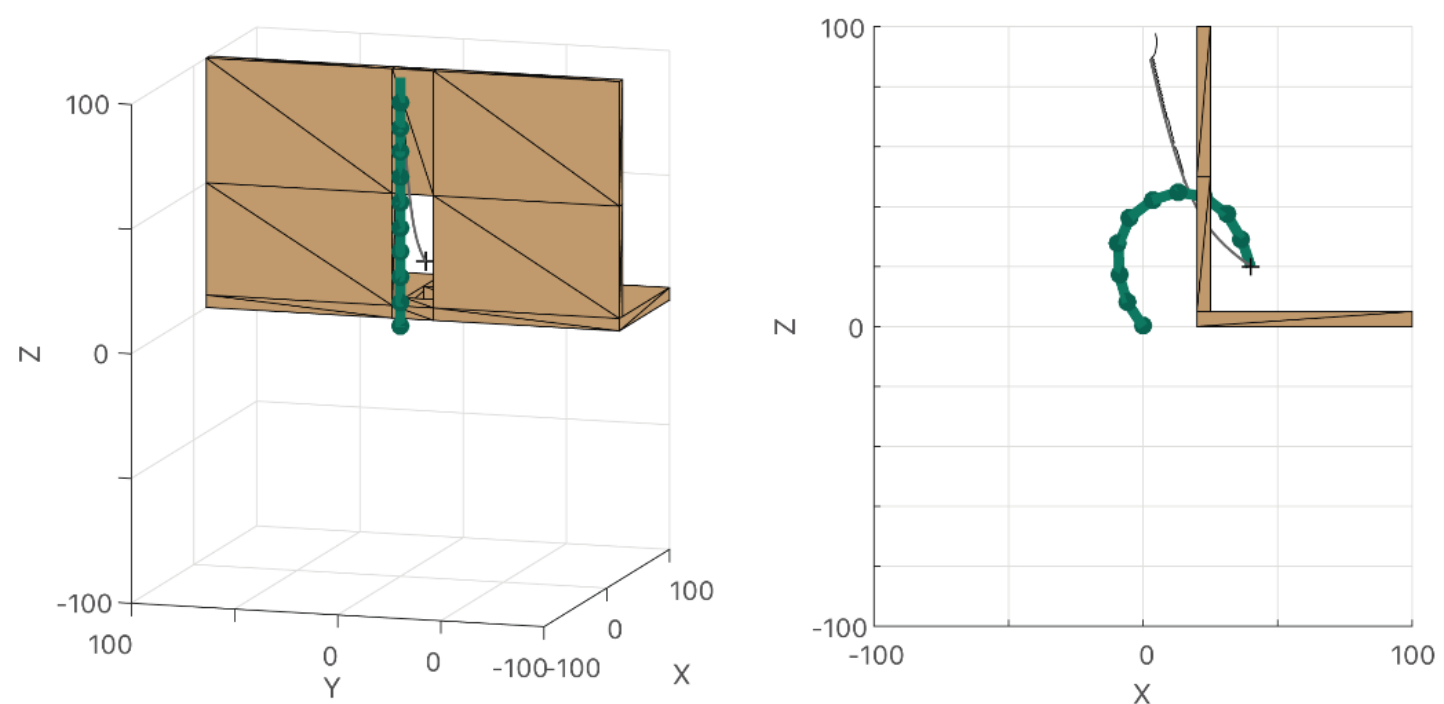

Figure 5.18. Motion planning for hyper-redundant robots in complex environments. No collisions occurred during the execution. (a) Initial robot configuration in an environment with two complex obstacles and representation of the desired position and a valid collision-free trajectory for the end effector. (b) Final robot configuration and path for the previous scenario. (c) Initial configuration, desired position, and collision-free trajectory for the end effector in other scenarios with walls and holes. (d) Final robot configuration and path for the previous scenario.

To conclude the simulation results, it has been tested the previous features of hyper-redundant robots all at once in order to study their performance (Figure 5.19). For example, a hyper-redundant robot with 9 joints is studied. A mechanical failure in joints 2 and 3 has been introduced. The end effector joint is both rotational and prismatic. Additionally, 9 consecutive spheres have been introduced in the working environment as obstacles that the robot has to avoid. The goal is to follow a linear trajectory with a desired 
orientation in all of its points with a precision of $0.1 \%$. Despite all these constraints, the algorithm works successfully for the given task.

a

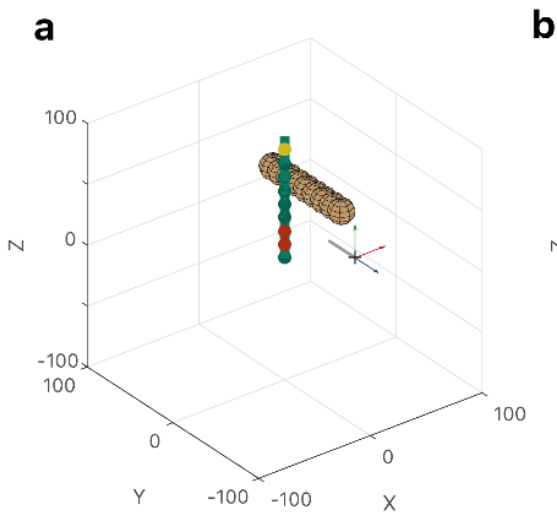

b

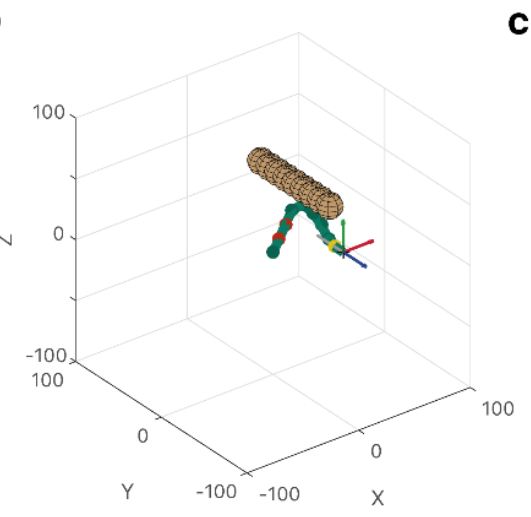

C

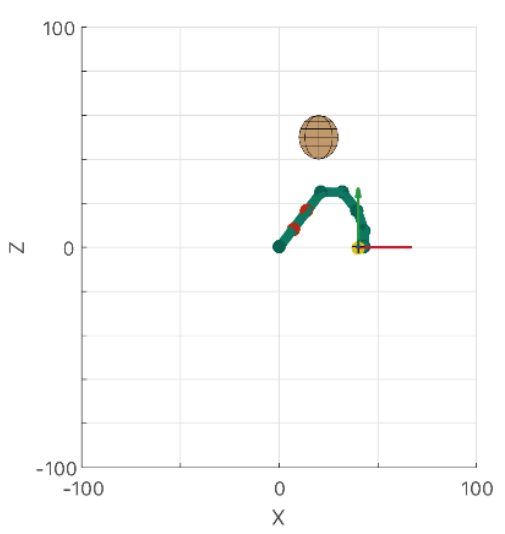

Figure 5.19. Use of the natural-CCD algorithm for resolution of a complex task with a lot of constraints. No collisions occurred during the execution. (a) Initial configuration of a robot with 9 rotational joints and 3 DOFs per joint. A mechanical failure is simulated in 2 joints (second and third from the base) and the last joint is also prismatic. The workspace includes 9 consecutive obstacles that have to be avoided. Then, the robot must conduct a linear trajectory with a given orientation. (b) Final robot configuration in a $3 D$ perspective. (c) Final robot configuration in a front view.

\subsubsection{Validation}

An application has been developed in MATLAB 2016a in Windows 10 to test the performance of the NaturalCCD. A computer featuring an Intel ${ }^{\circ}$ Core i7-4720HQ $(2.60 \mathrm{GHz})$ processor and $16 \mathrm{~GB}$ of RAM memory was used to execute the algorithm. As previously mentioned, the most important criteria to analyse the effectiveness of an inverse kinematics algorithm for hyper-redundant robots are the precision, computational time and quality of the solution.

Precision. Using the Natural-CCD algorithm, the end-effector of the robot will always converge to the desired location, if reachable, under a certain precision. This is why this parameter has been taken as an input variable requirement, whereas the computational time is the critical one to measure. It is worth noting that, the precision should be relative to the robot length. For example, it is not the same for a robot of 10 centimetres and a robot of 1 -meter length to reach a desired position with a precision of 1 millimetre. The precision in the first case should be $1 \%$ and in the second case $0.1 \%$. Precisions up to $10^{-7} \%$ have been defined without significantly increasing the computational time when the Natural-CCD algorithm is applied.

Computational time. This is usually the most critical variable when a hyper-redundant robot relies on a large number of DOF. One of the main reasons to have chosen the CCD as starting point is because it is a very simple and computationally efficient method. The Monte Carlo method has been used to study the computational time against the number of joints. A set of 100 random initial robot configurations and desired locations have been taken inside the reachable 3D workspace - a solid sphere of radius the length of the robot - to measure the computational time. This has been done for robots with different number of joints and DOF per joint. The precision was set $0.1 \%$ for all cases. Figure 5.20 shows that the computational times for one, two or three DOF per joint are quite similar. The differences among them are a result of the randomized input data. However, it is remarkable that the computational times are less than 1 second for robots of up to 300 DOF for both desired positions and desired positions and orientations. When robots of up to 1000 DOF are managed, it can be deduced that the computational time grows lineally with an increase of the number of joints, which is a huge advantage. 
a

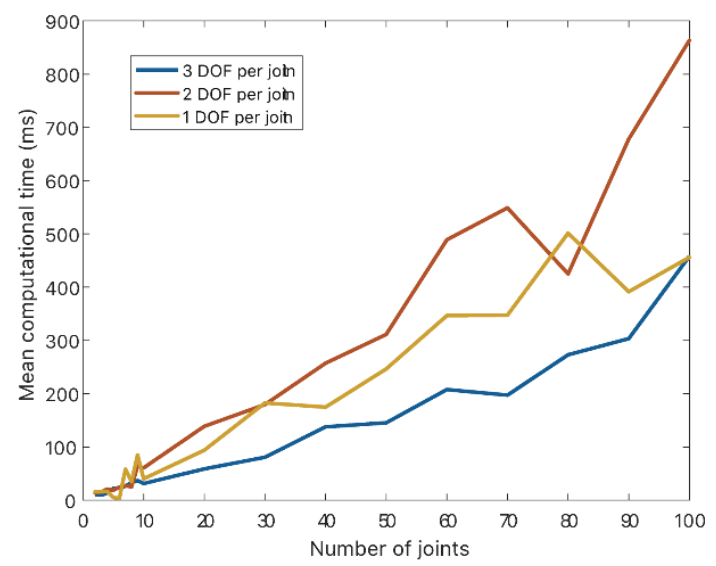

b

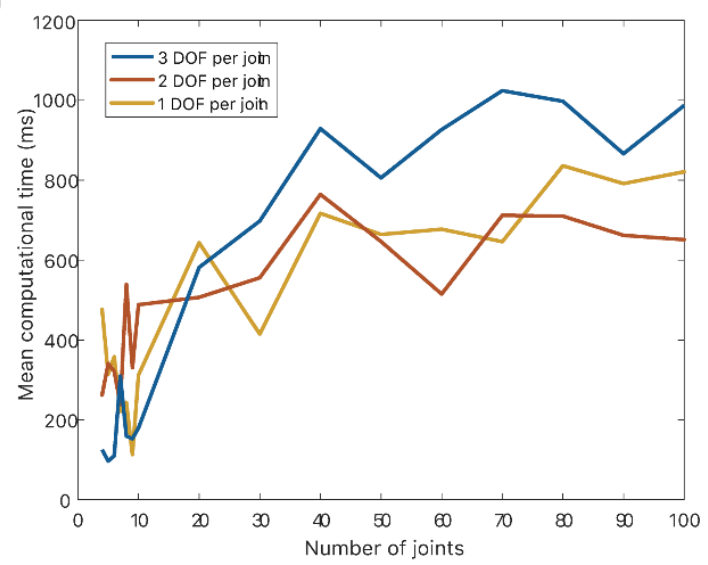

Figure 5.20. Mean computational time of the inverse kinematics problem resolution versus the number of joints for 1, 2, or 3 DOFs per joint (a) for given random positions (b) and for given random positions and orientations.

Solution optimality. A good-quality solution is one without self-collisions that maximizes the transmissibility of force, dexterity, rigidity, energy efficiency or availability of the joints range. This is, in other words, the one that best convey the strength to the end-effector, keep each joint as close as possible to its central position, minimize the speed, momentum and inertia of the joints, avoid singularities and maximize the skill of the end effector. First of all, the Natural-CCD ensures that the robot does not have any singularity or collide with itself. And also, it provides an incremental and uniform movement for all the joints of the robot. As a result, the robot shows an ability to easily move in a way that is biomimetic and fluid, giving place to coherent final configurations. 
The Natural-CCD performance can be compared with other similar studies from the state-of-the-art both qualitatively and quantitatively. This other methods are: Exhaustive methods [85], Pattern Search [85], Global Search [85], Genetic Algorithms [85], Simulated Annealing [85] and Particle-Swarm Optimization [78]. The CCD algorithm has not been included in the following comparisons because it does not offer acceptable solutions in terms of singularities, self-collisions and abrupt movements. First of all, due to the simplicity of the Natural-CCD, it manages a much higher number of DOF with acceptable computational times. It is a method with a very simple theoretical basis but with a high rate of convergence against other previous works. Despite the state-of-the-art methods, the precision of the presented algorithm is an input value that can be defined as a system requirement. It studies and manages the robot singularities, constraints and the joints movements that lead to appropriate and coherent solutions. About the computational time, the critical variable, the Natural-CCD provides solutions for the inverse kinematics problem in less than 1 second for robots of up to 300 DOF. Compared to the state-of-the-art methods that present computational times in order of seconds that increase exponentially against the number of joints, the Natural-CCD presents far lower ones that grow lineally against the number of joints (Figure 5.21).

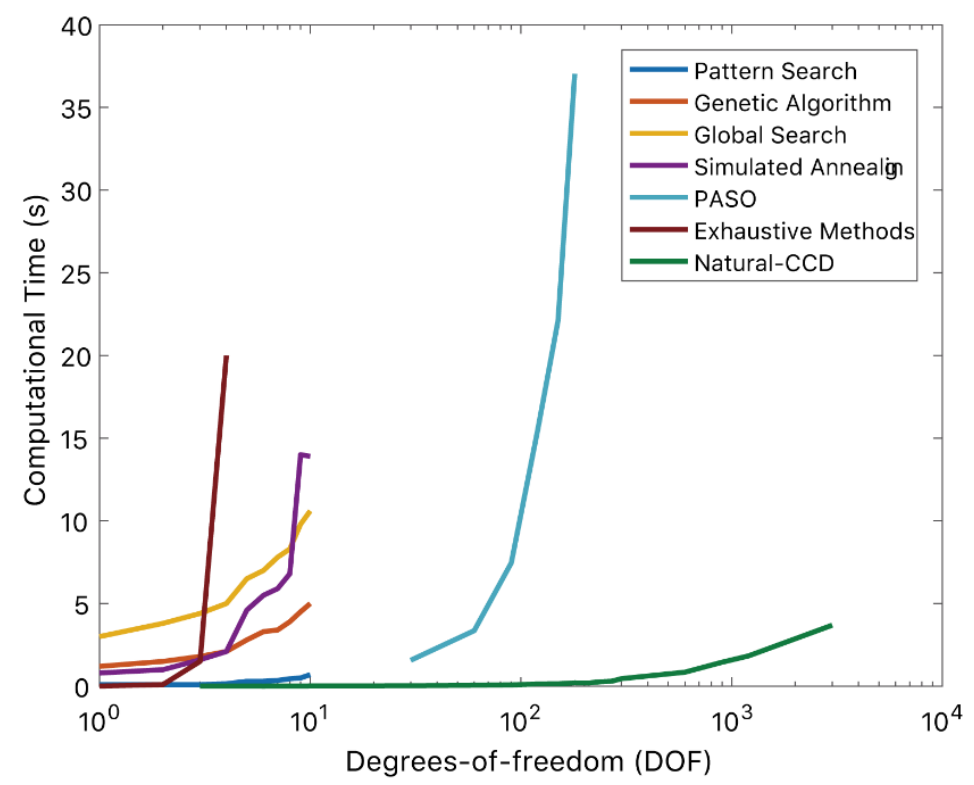

Figure 5.21. Computational time comparison of the state-of-the-art methods and the natural-CCD against the number of DOFs in a logarithmic scale.

In summary, the strengths of the algorithm compared to the state of the art are the simplicity, low computational cost, ability to establish high desired precisions and the quality of the solutions obtained. All of this could eventually lead to numerous potential benefits. First of all, the Natural-CCD can be a viable solution to solve the inverse kinematics problem of hyper-redundant robots with real-time requirements. Secondly, it is based in biomimetic movements that help to understand some shapes of nature like the Fibonacci spiral. And thirdly, it proposes a method to convert the obtained discrete solutions to continuous for soft robotic applications. 


\subsection{Extension to emerging behaviours}

As a remainder, the Natural-CCD algorithm uses three fundamental variables [pc, pe, pf] to manage the movements of highly-articulated robots. The core idea of this section work is to rearrange and reinterpret these three variables and use them to define a distributed control approach for hyper-redundant and growing robots to exploit their kinematic capabilities.

In this work, a behaviour is defined as a distinctive movement of a robot. In fact, the previously explained principles for the Natural-CCD algorithm constitute one of multiple behaviours. It allows the end-effector be attracted to an external reference, something useful to solve the inverse kinematics problem for hyperredundant and growing robots (Eq. 5.1). However, there are other possible behaviours that can be extracted by modifying the positions of the triangle formed by $\left[\mathbf{p}_{\mathbf{c}}, \mathbf{p}_{\mathbf{e}}, \mathbf{p}_{\mathbf{f}}\right]$ as vertices. This leads the emergence of a remarkable set of behaviours.

Let's have a closer look. In order to rotate each control point $P\left(\mathbf{q}_{\mathbf{i}}\right)$, there are a few relevant points to look at: (i) the starting point of the chain $P\left(\mathbf{q}_{\mathbf{1}}\right)$, (ii) a previous control point $P\left(\mathbf{q}_{\mathbf{i}-\mathbf{1}}\right)$, (iii) the current control position $P\left(\mathbf{q}_{\mathbf{i}}\right)$, (iv) a successive control point $P\left(\mathbf{q}_{\mathbf{i}+1}\right)$, and (v) the endpoint $P\left(q_{n}\right)$. Moreover, (vi) $\mathbf{Q} \in \mathrm{I} R^{m}$ will be used to represent any external reference to the kinematic chain (Figure 5.22).

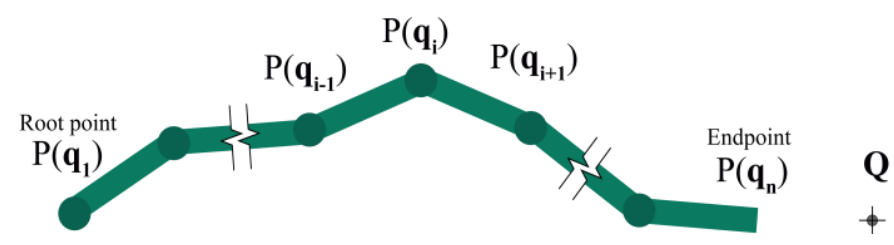

Figure 5.22. Relevant points in a kinematic chain and the location of an external reference $\boldsymbol{Q}$.

Then, it is possible to find different combinations of $\left[\mathbf{p}_{\mathbf{c}}, \mathbf{p}_{\mathbf{e}}, \mathbf{p}_{\mathbf{f}}\right]$ that can be assigned to these points. Each combination results in different values of $\theta_{i}$ (Eq. 5.2) and $\mathbf{d}_{\mathbf{i}}$ (Eq. 5.3), leading to different rotations on each control point and eventually to an overall different behaviour. It is possible to calculate the number of possible behaviours. Here, $\left[\mathbf{p}_{\mathbf{c}}, \mathbf{p}_{\mathbf{e}}, \mathbf{p}_{\mathbf{f}}\right]$ has dimension $k=3$, and the set of relevant points defined in Figure 5.22 has dimension $x=6$. Therefore, 20 distinctive behaviours $(B)$ emerge from k-combinations on the set of $x$-elements, as defined by Eq. 5.19.

$$
\left(\begin{array}{l}
x \\
k
\end{array}\right)=\frac{x !}{k !(x-k) !}
$$

Additionally, internal permutations of $\left[\mathbf{p}_{\mathbf{c}}, \mathbf{p}_{\mathbf{e}}, \mathbf{p}_{\mathbf{f}}\right]$ could lead to even more behaviours. However, it is important to highlight that only non-circular permutations lead to different behaviours. This is because of how rotation directions are calculated by using the cross product (Eq. 5.3). A circular permutation shifts all elements of a set by a fixed offset, with the elements shifted off the end inserted back at the beginning. To clarify this, the number of ways to arrange $k$ distinct objects along a fixed circle is

$$
\begin{array}{ll}
P_{k}=(k-1) ! & \text { Eq. } 5.20
\end{array}
$$

In the case $k=3$, there are 2 possible ways to arrange the permutation set: i.e. $\left[\mathbf{p}_{\mathbf{c}}, \mathbf{p}_{\mathbf{e}}, \mathbf{p}_{\mathbf{f}}\right]$ and $\left[\mathbf{p}_{\mathbf{c}}, \mathbf{p}_{\mathbf{f}}, \mathbf{p}_{\mathbf{e}}\right]$. As a result, any of the 20 behaviours $(B)$ will have their complementary $(\bar{B})$. Such complementary behaviours will change the sign of $\mathbf{d}_{\mathbf{i}}$ (Eq. 5.3) in every iteration since the cross product is anticommutative. Accordingly, a total of 40 behaviours can be obtained when the Natural-CCD algorithm is extended by different combinations of $\left[\mathbf{p}_{\mathbf{c}}, \mathbf{p}_{\mathbf{e}}, \mathbf{p}_{\mathbf{f}}\right]$, as seen in Table 5.3. 


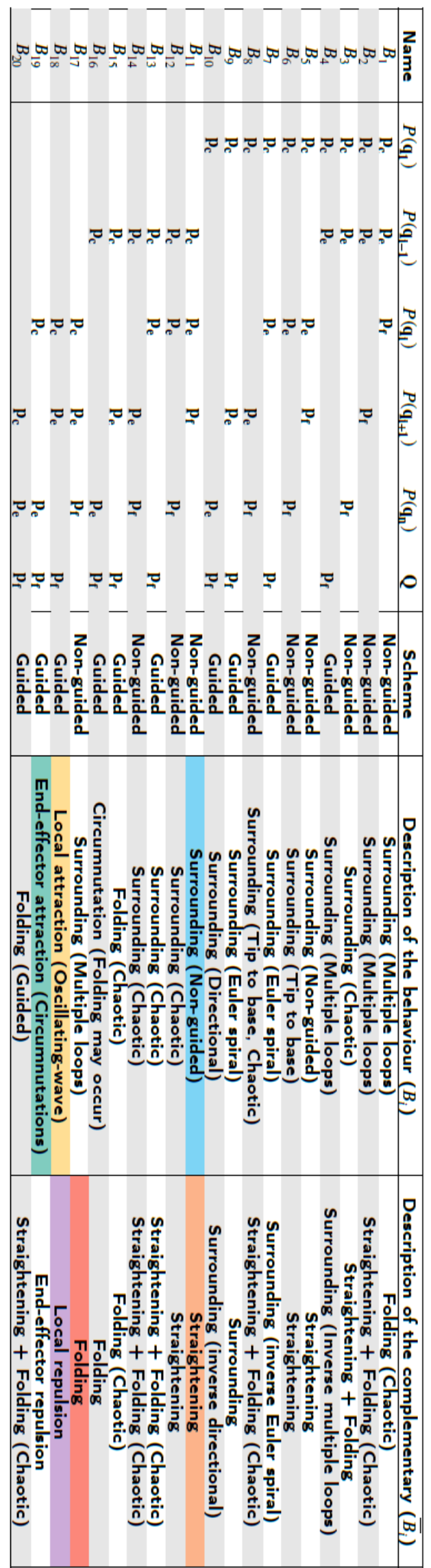


If any variable of a behaviour rely on an external reference ( $\mathbf{Q})$, they are classified as guided. If they passively perform a specific action relying only on their current morphology they are called non-guided (Table 5.3).

All these behaviours are result of applying the Natural-CCDalgorithm, which cyclically and uniformly moves all the control points by small increments. Hence, all behaviours emerge from differently distributed control schemes. Not all behaviours are functional and distinctive. For example, some of them like $B_{8}, B_{13}, B_{14}$ or $B_{15}$ seem to undergo chaotic motion, showing a sensitive dependence on the initial conditions. However, others are stable, distinctive and functional for hyper-redundant and growing robots.

Out of these 40 behaviours, some of the most relevant have been selected. They can be grouped in 6 different categories: Attraction (Section 4.1) and Repulsion (Section 4.2), Surrounding (Section 4.3) and Straightening (Section 4.4), Folding (Section 4.5) and Circumnutations (Section 4.6).

\subsubsection{Relevant behaviours}

\subsubsection{Attraction}

a

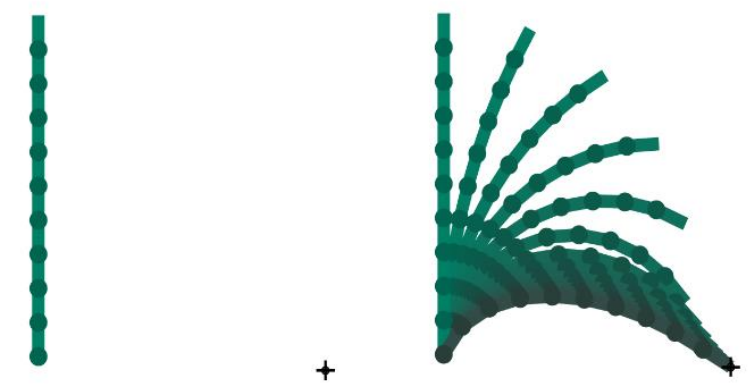

b
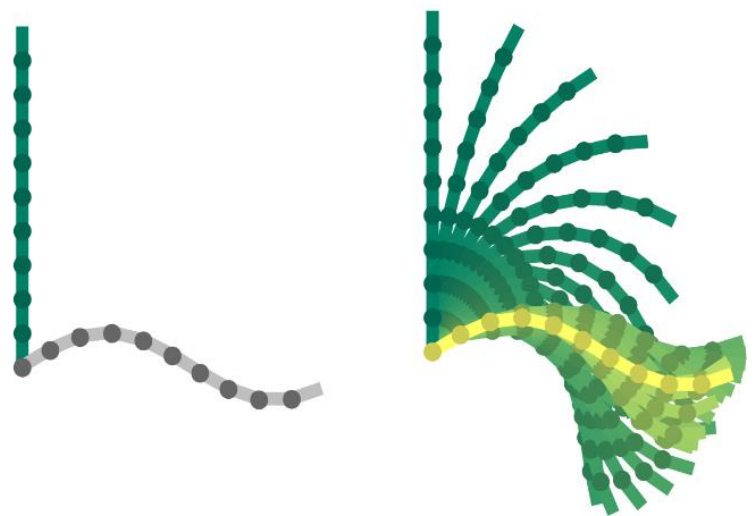

Figure 5.23. (a) The inverse kinematics problem requires a single reference to optimize the end-effector location. (b) Shape control requires a multi-variable reference (grey) to define the desired pose of the robot.

Some behaviours were found to attract the robot to an external input. For example, behaviour $B_{19}$, which is the original Natural-CCD algorithm described in Eq. 5.1, let the end-effector be attracted towards a desired reference, solving implicitly the inverse kinematics problem (Figure 5.23a).

Similarly, behaviour $B_{18}$ divides the inverse kinematics problem into many sub-problems. Every control point of the robot is attracted to different targets. This kind of behaviour is usually called shape control [13]. 
Consequently, a multi-variable input is needed to define the robot configuration (Figure 5.23b), rather than just one variable for the end-effector (Figure 5.23a). In other words, by performing a shape control, the reference needs to change from $\mathbf{Q} \in \mathrm{I} R^{m}$ (Eq. 1.2b) to $\mathbf{Q} \in \mathrm{I} R^{n}$, where $m$ is the dimension of the desired location and $n$ the number of degrees-of-freedom. As a consequence, the configuration of the robot $\mathbf{q} \in \mathrm{I} R^{n}$ will not longer be underdetermined (Eq. 1.2b), but fully determined (Eq. 5.21). Thus, the kinematic hyperredundancy becomes debatable by using this approach, since in this case

$$
\operatorname{dim}(\boldsymbol{q})=\operatorname{dim}(\boldsymbol{Q})=n \quad \text { Eq. } 5.21
$$

Following this idea, the initial definition of $\mathbf{p}_{\mathbf{c}}$ stays as in the original Natural-CCD algorithm, $\mathbf{p}_{\mathbf{e}}$ becomes the position of the successive control point of the current state of the robot, and $\mathbf{p}_{\mathbf{f}}$ is now located at the reference $\mathbf{q}^{\prime}$ (Eq. 5.22).

$$
\begin{aligned}
& \boldsymbol{p}_{\boldsymbol{c}}=P\left(\boldsymbol{q}_{\boldsymbol{i}}\right) \\
& \boldsymbol{p}_{\boldsymbol{e}}=P\left(\boldsymbol{q}_{\boldsymbol{i}+\mathbf{1}}\right) \\
& \boldsymbol{p}_{\boldsymbol{f}}=\boldsymbol{Q}=P\left(\boldsymbol{q}_{\boldsymbol{i}+\mathbf{1}}^{\prime}\right)
\end{aligned}
$$

Eq. 5.22

The challenge of shape controls is to find the appropriate input. Once a reference is selected, there is more than one method to perform the shape control. For example, [11] proposed a fast and converging method by decoupling the reference and relocating the inputs to the current state of the robot (Figure 5.24a).
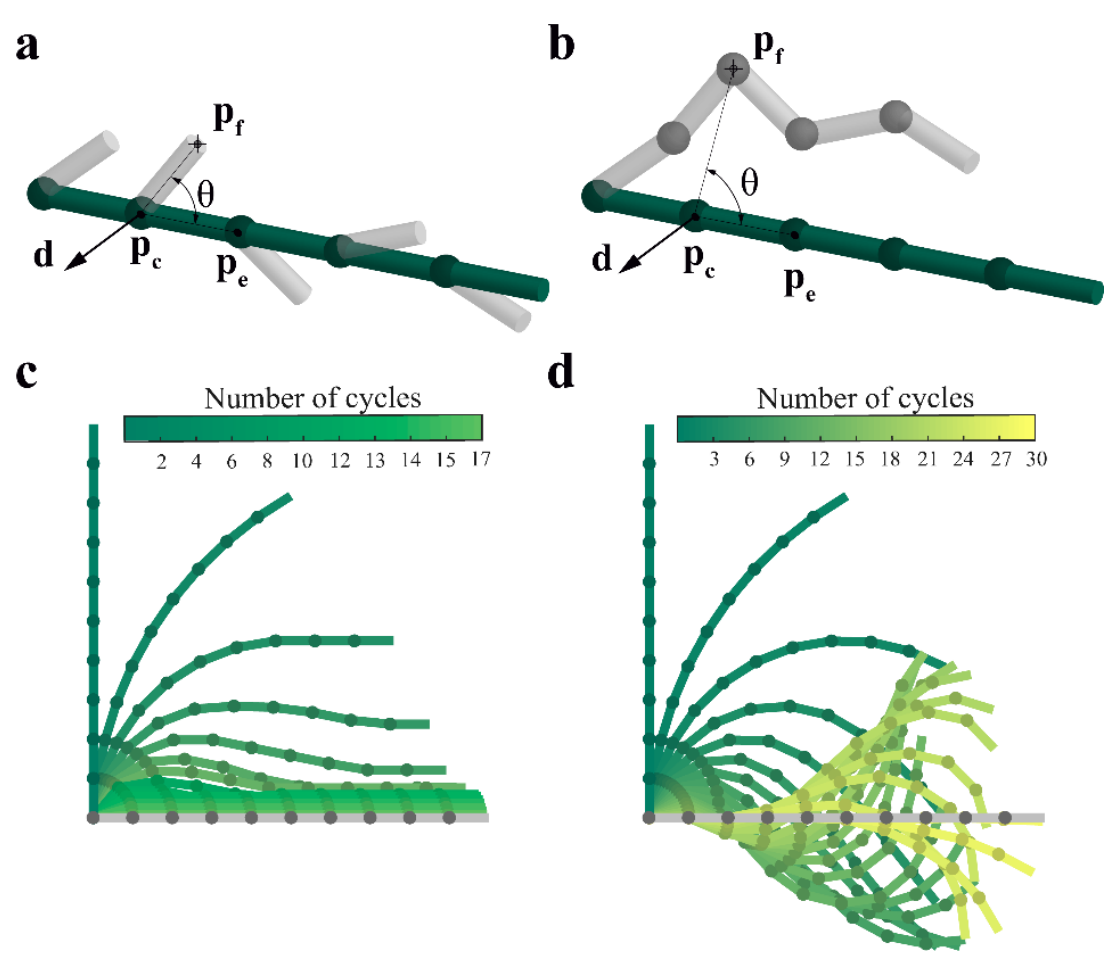

Figure 5.24. Different strategies for to perform a shape control. The current state of the robot (green) and the reference (grey) are the same in both cases. (a) The decoupled strategy (c) leads to a fast converging solution. And (b) the coupled strategy (d) leads to an oscillating-wave movement.

This strategy let the control points move independently from their predecessors, hence, the kinematic chain is decoupled. In this scenario velocities can be easily managed to achieve coordinated and synchronous movements (Figure 5.24c). 
However, if the same robot pose reference is sequentially coupled (Figure 5.24b), then an oscillating-wave movement will emerge (Figure 5.24d). This oscillation is the result of an accumulation of the incremental angles given by the movement of each control point added to the respective ones from their predecessors. Therefore, this behaviour relies on the cyclical and incremental rotation of every control point using the Natural-CCD. This approach also converges the pose to the solution since the movement of the robot will gradually converge from the base to the tip following the response of an underdamped system.

The oscillating-wave behaviour does not converge as quickly as the decoupled one. However, it is compatible with prismatic capabilities and the addition of new control points as a result of growth (Figure 5.3).

By using this methodology, growing robots could take advantage of their movement speed and variable length to obtain a desired frequency for the oscillating-wave behaviour. This can be understood in the context of the Marsenne's law [261], which settles that the fundamental frequency of oscillation $(f)$ of a stretched string is directly proportional to the speed of propagation $(v)$ and inversely proportional to its length $(L)$ (Eq. 5.23). Under this perspective, the kinematic chain of the robot can be understood as a loose string anchored on one end that cyclically propagates its movement until it conforms towards the reference.

$$
f=\frac{v}{2 L}
$$

Therefore, it is possible to control the frequency of the oscillating-wave behaviour $\left(B_{18}\right)$ by controlling the length, the velocity or the number of control points of growing robots. For example, if a manipulator grows through adding more control points and the velocities and the length remain constant, then the frequency of the wave will proportionally increase (Figure 5.25). Hence, if this behaviour is required to persist in time, then the reference location has to dynamically change as the robot continues attempting to reach it. Otherwise, this approach will converge to the solution. Applications of this kind of behaviour vary from breaking through unstructured environments, enveloping the robot pose towards remote trajectories or performing follow-the-leader guidance, as explained in Section 5.2.2.

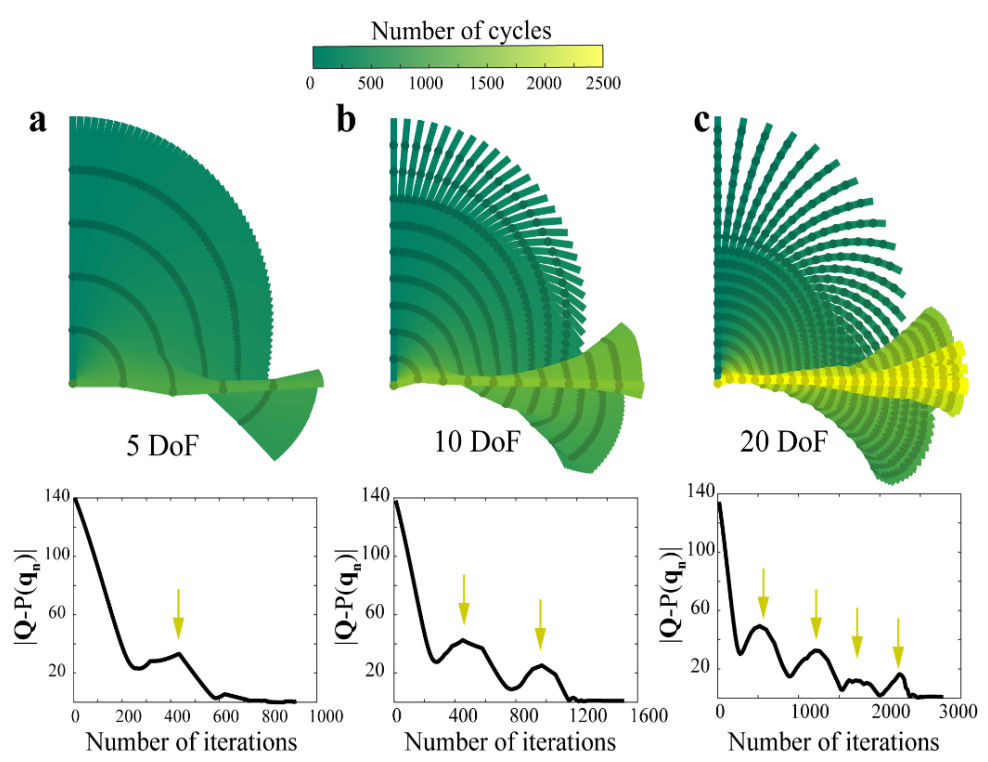

Figure 5.25. Oscillating-wave movement of robots with the same length and different degrees-of-freedom (DoF) moving at the same velocity. Also, convergence between the end-effector and the desired point $\left\|\boldsymbol{Q}-P\left(\boldsymbol{q}_{\boldsymbol{n}}\right)\right\|$. (a) 5 control points lead to 1 period, (b) 10 control points lead to 2 periods and (c) 20 control points lead to 4 periods. 


\subsubsection{Repulsion}

Repulsion behaviours are the complementary of the attraction ones. For example, the complementary of the end-effector attraction $\left(B_{19}\right)$ gives place to an end-effector repulsion $\left(\bar{B}_{19}\right)$. With this approach, every module of the robot will try to get the end-effector as far away as possible from the reference point. This could be used to steer the robot's end-effector away from moving objects such as humans, other robots or obstacles. However, it does not guarantee the repulsion of objects from all the points in the kinematic chain. This can be added including the complementary behaviour of the local attraction $\left(B_{18}\right)$, called local repulsion $\left(\bar{B}_{18}\right)$ (Figure 5.26). This behaviour $\bar{B}_{18}$ is defined by swapping the values of $\mathbf{p}_{\mathbf{e}}$ and $\mathbf{p}_{\mathbf{f}}$ and by selecting $\mathbf{Q}$ as the closest point on the surfaces of an external object that needs to be avoided (Eq. 5.24).

$$
\begin{aligned}
& \boldsymbol{p}_{\boldsymbol{c}}=P\left(\boldsymbol{q}_{\boldsymbol{i}}\right) \\
& \boldsymbol{p}_{\boldsymbol{e}}=\boldsymbol{Q} \in I R^{n} \\
& \boldsymbol{p}_{\boldsymbol{f}}=P\left(\boldsymbol{q}_{\boldsymbol{i}+\mathbf{1}}\right)
\end{aligned}
$$

Eq. 5.24

a

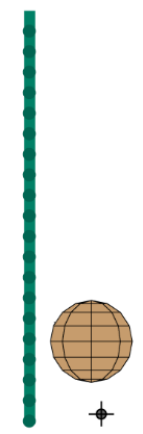

b

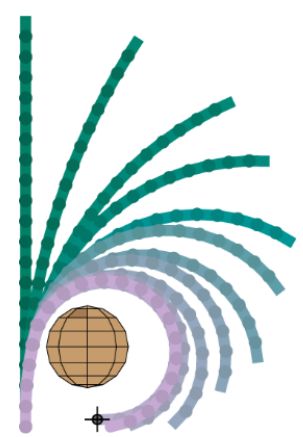

Figure 5.26. (a) Initial configuration of the robot, an static obstacle and the desired end-effector location. (b) The robot configuration is locally repelled to avoid the obstacle, but still reaches the reference with its end-effector.

This behaviour can be activated when a external event appears, e.g. when approaching an object. Moreover, this activation can also be applied gradually as the distance between the robot and the object is reduced. Therefore, this behaviour can be very useful for obstacle avoidance in real-time (Figure 5.27a).

If one object moves towards the robot arm, it will be repelled using this distributed behaviour while the rest of the robot still tries to achieve the target (Figure 5.27b). However, growing robots can even extend their length when contacting external objects so the rest of the pose remains unaffected (Figure 5.27c). Therefore, they show a much higher adaptability to their surroundings.

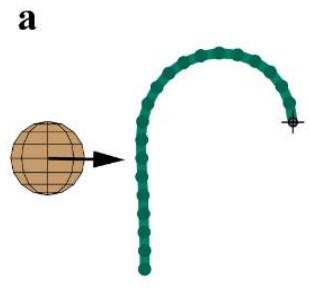

b

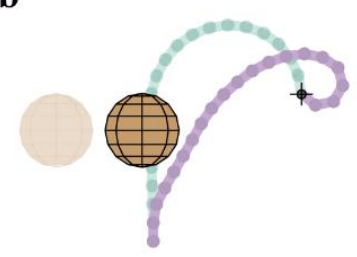

c

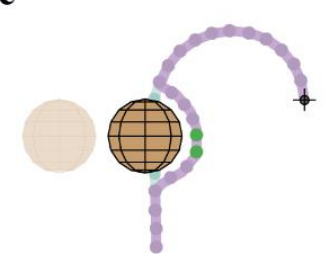

Figure 5.27. (a) Obstacle approaching to a static configuration of the robot. (b) The robot configuration is automatically adapted to locally repel the dynamic object. (c) A growing robot can add new control points (green) to easily avoid the same obstacle while the pose remains static and more energy-efficient. 


\subsubsection{Surrounding}

Many behaviours exhibit surrounding properties like $B_{6}, B_{10}$ or $B_{11}$. The ones relying on an external reference $(\mathbf{Q})$, the guided strategies, can control the direction of the robot to surround a predefined object. However, non-guided behaviours let the robot move relying only on its current shape.

For example, behaviour $B_{10}$ is guided and shows a directional surrounding, which bends the kinematic chain until the base and the tip of the robot are aligned with the reference, independently from the rest of the body. Note that this strategy is not feasible without additional kinematic constraints, such as self-collisions or a maximum bending angle, since otherwise the robot could collide with itself. This behaviour aligns the end-effector with the position of the base and the external reference (Q). As a result of this geometry, if that reference is expressed in polar coordinates, the behaviour will only change by increments in the angular coordinate, remaining unaffected by the radial distance. Accordingly, behaviour $\bar{B}_{10}$ will move in the opposite direction of $B_{10}$.

Similarly, behaviours $B_{9}$ and $\bar{B}_{9}$ replicate the same idea of $B_{10}$ and $\bar{B}_{10}$. The difference here is that every control point in the kinematic chain attempts to align itself between the robot base and the external reference Q, resulting in shapes that remind of Euler spirals.

Regarding non-guided strategies, they could be very useful to grab and release objects without any prior knowledge of the morphology or exact location. For example, behaviour $B_{11}$ will rotate every joint in order to align its successors to their predecessors (Eq. 5.25).

$$
\begin{aligned}
& \boldsymbol{p}_{\boldsymbol{c}}=P\left(\boldsymbol{q}_{\boldsymbol{i - 1}}\right) \\
& \boldsymbol{p}_{\boldsymbol{e}}=P\left(\boldsymbol{q}_{\boldsymbol{i}}\right) \\
& \boldsymbol{p}_{\boldsymbol{f}}=P\left(\boldsymbol{q}_{\boldsymbol{i}+\mathbf{1}}\right)
\end{aligned}
$$

As a result, a hyper-redundant robot with kinematic constraints will exhibit surrounding movements. In the cases there is no object it simply coils up (Figure 5.28).

a

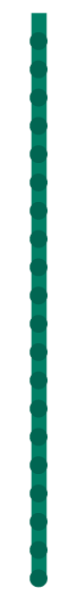

b

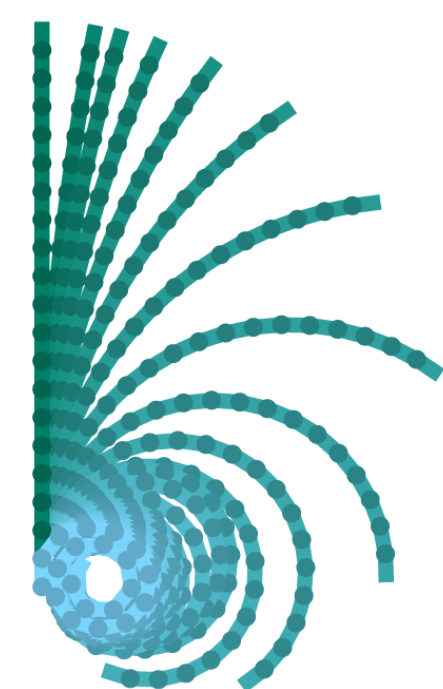

c

Figure 5.28. (a) Initial configuration of the robot. (b) Intermediate configurations of the surrounding behaviour. (c) Final configuration with self-collision constraints.

On the other hand, if the robot makes contact with an object while using this behaviour, then it will surround it (Figure 5.29a). These movements are very similar to the palmar grasp reflex, found in humans and primates [262]. 
Other non-guided alternatives, such as behaviour $B_{6}$ will tend to bend the kinematic chain until the tip approaches the base and encloses the robot pose. This strategy is stable and does not need any kinematic constraints in order to appear, as opposed to $B_{10}$ or $B_{11}$.

The surrounding behaviour can also be exploited by growing robots. First, they can surround objects that are bigger and farther away since their workspace increases as their body also extends. And second, they can improve their maneuverability in order to grasp more tightly complex objects (Figure 5.29).

$\mathbf{a}$

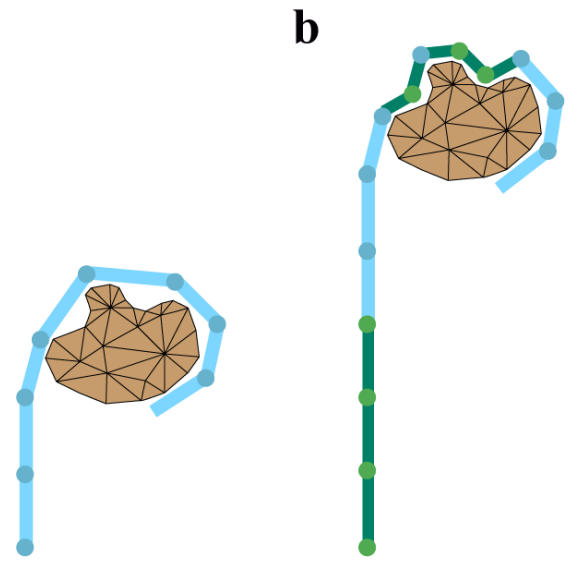

Figure 5.29. (a) Object surrounding performed by a regular hyper-redundant robot. (b) A growing robot can reach farther objects and adjust its shape to tight around irregular morphologies.

Moreover, growing robots could potentially take advantage of this behaviour by extending through branching or arborescent structures (Figure 5.30a). This scheme, inspired by plants and roots, could be used to facilitate grabbing complex objects by increasing friction through adding more contact points (Figure $5.30 \mathrm{~b})$.

$\mathbf{a}$

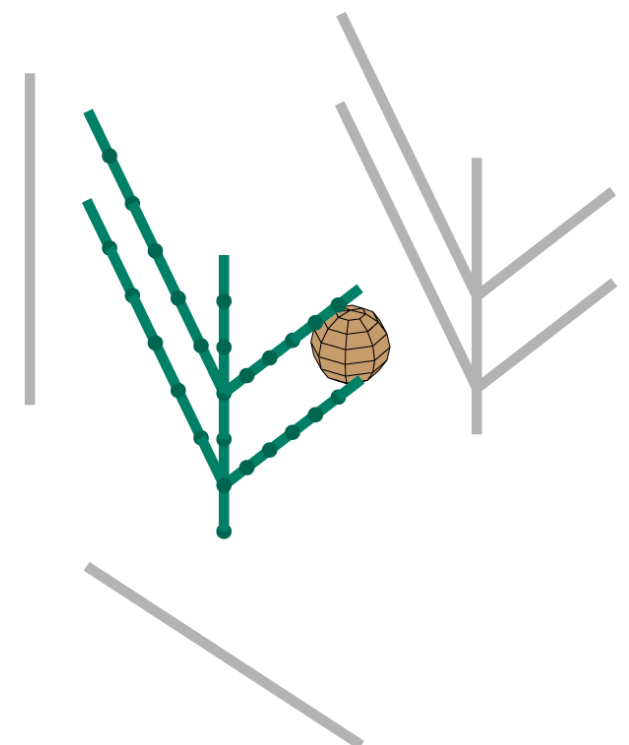

b
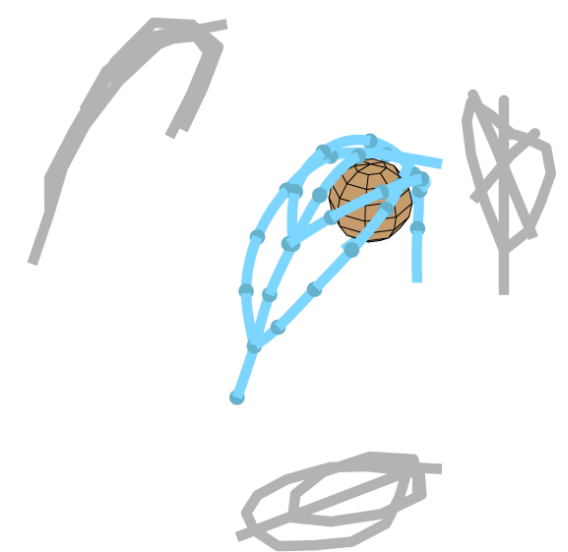

Figure 5.30. (a) A growing robot could customize their structure using branches depending on the application. (b) This structure allows the robot to grab complex objects by using the surrounding behaviour on each arm independently. Shadows are depicted in grey to ease the spatial understanding. 


\subsubsection{Straightening}

Similarly to attraction and repulsion, the complementary behaviours of surrounding is called straightening because it tends to align all the control points of the robot.

For example, behaviour $\bar{B}_{11}$ (Eq. 5.26) is a non-guided behaviour that moves all the control points until the pose is completely straight (Figure 5.31).

$$
\begin{aligned}
& \boldsymbol{p}_{\boldsymbol{c}}=P\left(\boldsymbol{q}_{\boldsymbol{i}-\mathbf{1}}\right) \\
& \boldsymbol{p}_{\boldsymbol{e}}=P\left(\boldsymbol{q}_{\boldsymbol{i}+\mathbf{1}}\right) \\
& \boldsymbol{p}_{\boldsymbol{f}}=P\left(\boldsymbol{q}_{\boldsymbol{i}}\right)
\end{aligned}
$$

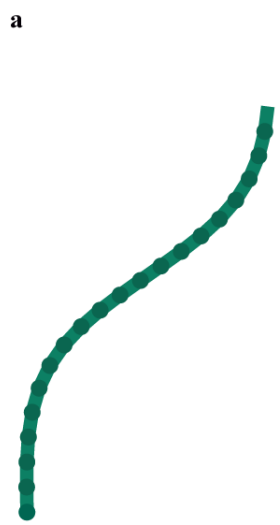

b

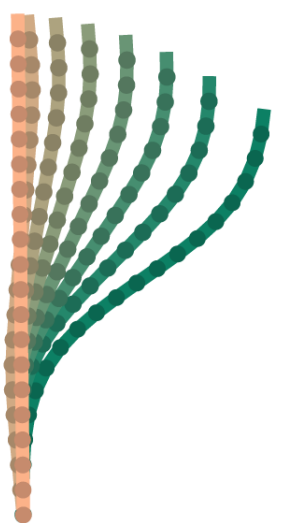

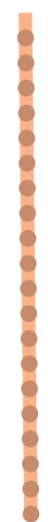

Figure 5.31. (a) Initial configuration of a hyper-redundant robot. (b) The straightening behaviour aligns all the control points. (c) Final configuration.

The resulting configuration let the end-effector reach the limits of the workspace, but it reduces the mobility of the robot. However, this behaviour could be used to increase the rigidity of the robot since the resulting pose tends to be a boundary singularity [3].

Furthermore, this behaviour can be used to release grasped objects. Additionally, it can be very useful to straighten partial sections or even the whole-body of the robot if needed. Moreover, it can be used by growing robots to align their pose in order to retract its body after being extended.

\subsubsection{Folding}

The folding behaviours emerged as complementaries of multiple behaviours. This kind of movements retract the robot configuration following a zigzag pattern. Interestingly, they are not complementary to the a straightening behaviour as initially expected but rather they are complementary to completely different behaviours.

Two different distinctive folding behaviours were found. Firstly, behaviour $\bar{B}_{17}$, which is non-guided, so it folds the robot depending on the initial configuration of the robot (Eq. 5.27).

$$
\begin{aligned}
& \boldsymbol{p}_{\boldsymbol{c}}=P\left(\boldsymbol{q}_{\boldsymbol{i}}\right) \\
& \boldsymbol{p}_{\boldsymbol{e}}=P\left(\boldsymbol{q}_{\boldsymbol{n}}\right) \\
& \boldsymbol{p}_{\boldsymbol{f}}=P\left(\boldsymbol{q}_{\boldsymbol{i}+\mathbf{1}}\right)
\end{aligned}
$$


For instance, when the initial configuration is straight and the initial singularities are managed, the robot performs the folding movements, shown in Figure 5.32.

The second folding behaviour is guided $\left(B_{16}\right)$. In this scenario the robot aligns the folding direction following an external reference. In contrast to the non-guided folding $\left(\bar{B}_{17}\right)$, this behaviour is the result of a propagating wave in the chain that increases its frequency until the robot is completely folded. Alternatively, behaviour $B_{20}$ also exhibits folding capabilities. Interestingly, at the same time, it tries to reach the external reference. In all the cases, they need to be programmed in combination with kinematic constraints, otherwise, the robot pose will tend to collide with itself.

The folding strategy can be very interesting for hyper-redundant robots when their long bodies need to efficiently retract after grasping an object, or to become very compact in order to improve their portability by increasing their rigidity and reducing their inertia. Moreover, if the robot is composed by elastic elements, it could be used to accumulate energy in the body and release it in a sudden manner.

Growing robots often exhibit irreversible extension capabilities, being unable to shorthen their length. Thus, this strategy can be very interesting to retract their body.

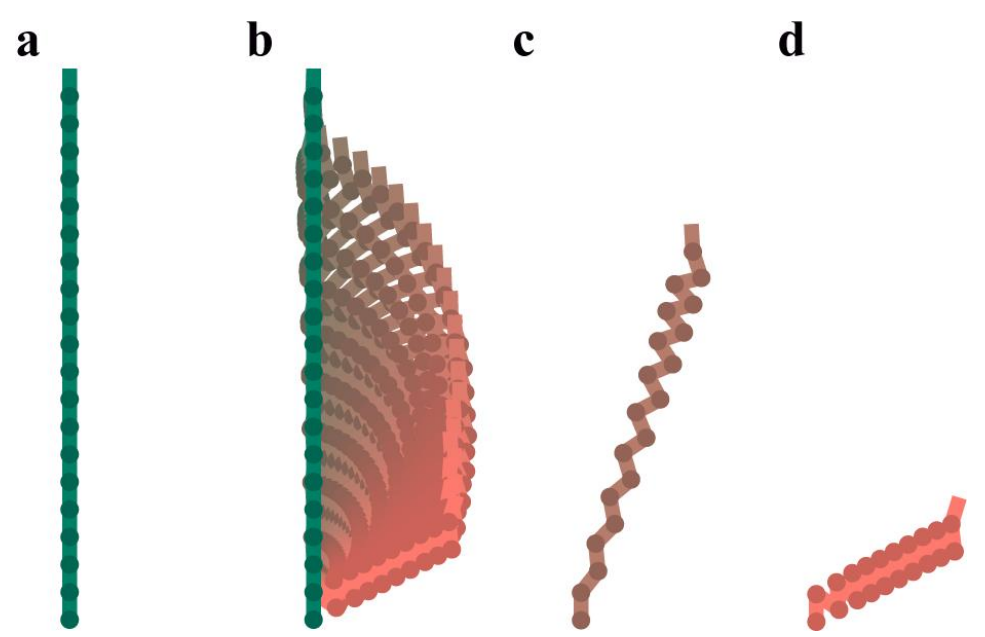

Figure 5.32. (a) Initial configuration of a hyper-redundant robot. (b) The folding behaviour retracts the configuration. (c) Intermediate configuration. (d) Final configuration.

\subsubsection{Circumnutations}

In botany, nutation is the bending movement executed by some plant organs by which the part is inclined successively in various directions. Specifically, a circumnutation can be described as a successive helical movement widespread among climbing plants. The origin of this behaviour in nature is still unclear [263], but it is still useful in the context of hyper-redundant and growing robots.

In this work, circumnutations appear when the end-effector attraction behaviour $\left(B_{19}\right)$ is used to solve the inverse kinematics problem (Eq. 5.1) over a moving reference (Figure 5.33). Technically, they were found when every point of a kinematic chain rotates to align the tip towards a moving reference, perpendicular to the plane that contains the robot configuration. 
a

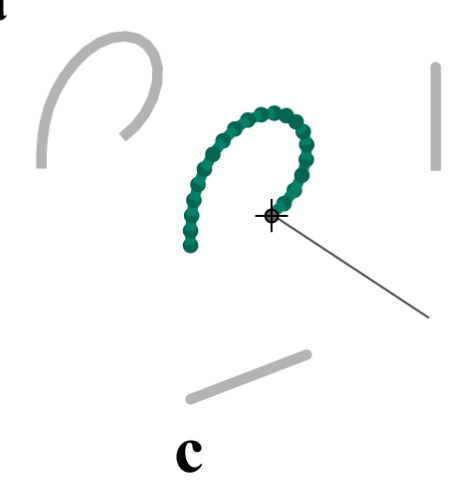

b

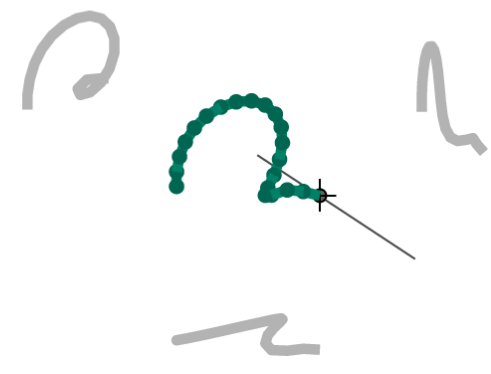

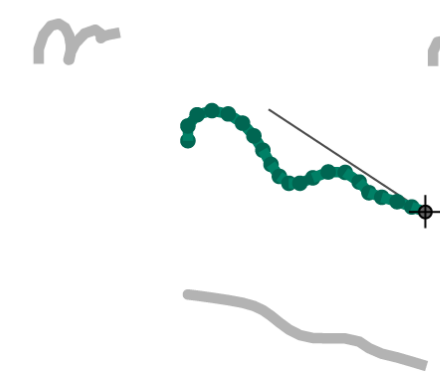

Figure 5.33. (a) Initial configuration. (b) The end-effector follows a straight line, perpendicular to the robot configuration using the end-effector attraction behaviour. (c) And the resulting movement corresponds to a helical circumnutation. Shadows are depicted in grey to ease the spatial understanding.

In nature, these movements of growing structures can be explained from an exogenous perspective. In fact, it is widely known that a lot of plants orient their tip to move towards the sun direction, which similarly constitutes a moving reference along in the sky.

Alternatively, behaviour $B_{15}$ follows the same principles as $B_{19}$. However, in this case, the robot keeps twisting its configuration following the helical circumnutation, until it retracts its body. Thus, it can be considered as a combination of the helical circumnutation and the folding behaviours. This approach could potentially be used to squeeze objects or to accumulate elastic energy like a spring does.

Applications of this behaviour vary from anchoring the robot to different shapes to grasping objects. Similarly to the results presented in Figure 5.25, growing robots could take advantage of this behaviour by extending their length in order to control the magnitude, period or shape of the helical circumnutations. Compared to regular hyper-redundant robots, this feature grants a much higher flexibility to interact with different shapes in unstructured environments.

\subsubsection{Motion strategies}

This section will provide simulation results from the application of the previously explained behaviours in various relevant scenarios. The different approaches to exploit such behaviours will be denominated motion strategies.

This work proposes 4 motion strategies. (i) Time-based strategies $\left(A_{1}\right)$ provides the possibility to switch behaviours over time to obtain complex configurations. (ii) Section-basedstrategies $\left(A_{2}\right)$ state that different behaviours can be applied to different sections of the kinematic chain. (iii) Event-based strategies $\left(A_{3}\right)$ propose that behaviours can be activated or deactivated based on environmental events. (iv) Combined 
strategies $\left(A_{4}\right)$ let behaviours be obtained from alternating different ones in non-consecutive control points of the kinematic chain. These motion strategies can even be simultaneously applied to perform more complex tasks.

Next, two scenarios will be described to illustrate the application of these motion strategies. In the first application, a hyper-redundant manipulator is required to perform the follow-the-leader strategy for inspection purposes while an external event hinders its completion (Section 5.1). This scenario illustrates the use of section-based $\left(A_{2}\right)$ and event-based $\left(A_{3}\right)$ strategies, making use of local attraction and repulsion behaviours (Section 4.1 and 4.2). Subsequently, in the second application, a growing robot is required to grab a distant object in the space (Section 5.2). This scenario illustrates the time-based $\left(A_{1}\right)$ and combined $\left(A_{4}\right)$ motion strategies by using the surrounding, folding and straightening behaviours (Sections 4.3, 4.5 and 4.4).

\subsubsection{Section and Event-based strategies}

A follow-the-leader strategy provides a trajectory as input that has to be followed by the end-effector and by the rest of the robot body as well. As a result, the manipulator follows a three-dimensional route with its body. This is very useful to navigate through narrow spaces, e.g. for search and rescue or inspection purposes (Figure 5.34).

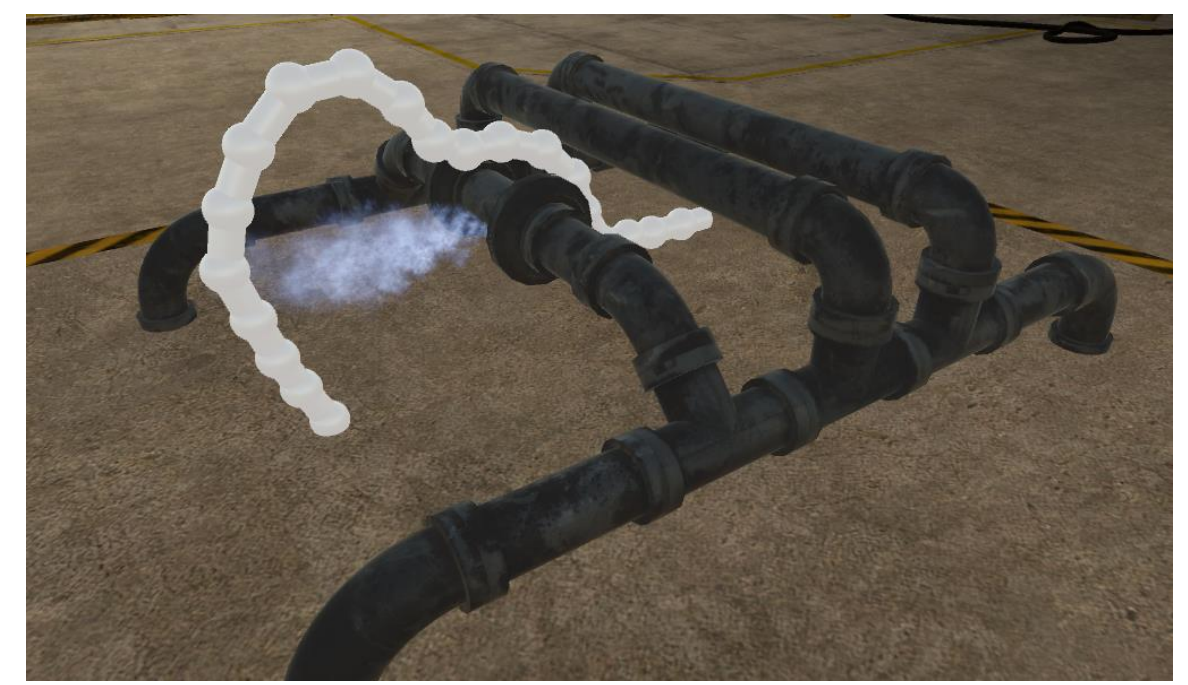

Figure 5.34. Hyper-redundant robot guided using the follow-the-leader control through a set of pipes for inspection by the sectionbased approach $\left(A_{2}\right)$. If there is a gas leak, the robot can use the event-based approach $\left(A_{3}\right)$ to locally repel it.

However, if the robot is a manipulator robot with a fixed base, then the problem has additional constraints and only some of the control points of the robot will be able to follow the desired trajectory. Therefore, it is crucial to design an appropriate motion strategy in order to find the desired input reference in every iteration.

In order to achieve the follow-the-leader strategy for a manipulator with a fixed base (Figure 5.35a), it will be necessary to apply a section-based approach $\left(A_{2}\right)$. The tip section of the robot (see yellow region in Figure $5.35 \mathrm{~b}$ ) will have to be attracted by the desired trajectory while, at the same time, restricted by the length of the base section (see green region in Figure 5.35b).

Thus, the local attraction behaviour $\left(B_{18}\right)$ will be applied to the tip section to command the movements of each control point independently. The higher the number of degrees-of-freedom, the higher the versatility of the robot configuration for complex trajectories. On the other hand, the section closer to the base will use 
behaviour $B_{19}$ to get the robot as closer as possible to the trajectory despite having the base in a fixed position (Figure 5.35b).

a

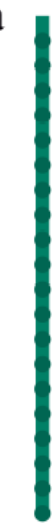

c

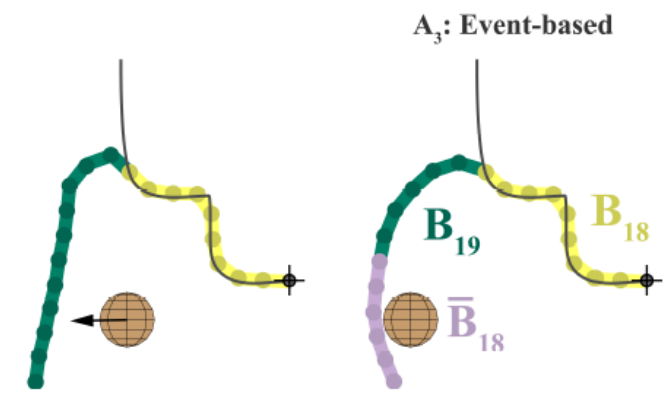

Figure 5.35. (a) Initial robot configuration and follow-the-leader trajectory. (b) The section-based approach $\left(A_{2}\right)$ divides the robot in two sections with different attraction behaviours $\left(B_{19}\right.$ and $\left.B_{18}\right)$. (c) If an external perturbation takes place, (d) the event-based approach $\left(A_{3}\right)$ will readjust the shape of the robot using the local repulsion behaviour $\left(\bar{B}_{18}\right)$.

Finding the limit between the two sections is non-trivial because the robot has constraints and the trajectory changes over time. In Figure 5.35, the end-effector was prioritized to reach the end of the trajectory. Based on this assumption, the number of control points of the ending section was maximized.

Additionally, after reaching the final configuration, a moving obstacle was approaching the robot while performing the previous motion strategy (Figure $5.35 \mathrm{c}$ ). The local repulsion behaviour $\left(\bar{B}_{18}\right)$, was then applied only to the closest control points to the perturbation. Thus, the event-based approach $\left(A_{3}\right)$ was employed. Growing robots could deal with this scenario even better as shown in shown in Figure 5.27c. But hyper-redundant ones can also readjust their body to some degree, barely influencing subsequent sections in the robot body (Figure 5.35d).

In serial manipulators, the movement of subsequent control points depend on the movement of their predecessors in the kinematic chain from the base to the tip. Similarly, subsequent behaviours will also rely on their predecessors to obtain a desired output. From this perspective, the resulting robot can be understood as a set of different manipulators sequentially appended to each other performing different behaviours, but working altogether towards the same objective.

This scenario was intentionally designed to be very specific and complex. It consists on performing an inspection guidance while also having external changing perturbations. Previous control techniques from the state-of-the-art are designed for each individual application, so they are not usually able to adapt to unpredictable events. Selected fundamental behaviours and motion strategies show a remarkable flexibility to successfully achieve complex tasks like the one presented. 


\subsubsection{Time-based and Combined strategies}

Extension capabilities performed by growing robots can be very useful to reach remote targets such as debris or samples in the space environment. Sometimes, those targets are delicate (e.g. satellites) and some of the previously explained behaviours can be used to gently pick and place objects exploiting the robot's shape. Hence, there is no need of using additional end-effector tools like grippers (Figure 5.36).

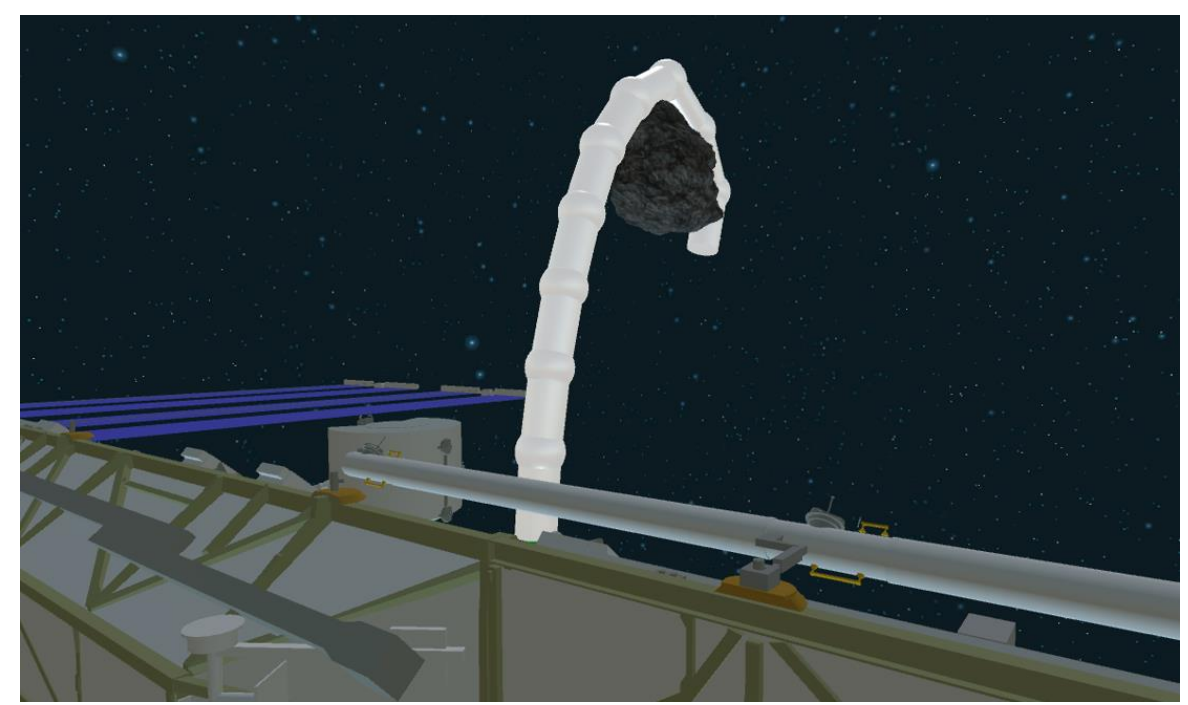

Figure 5.36. A growing robot taking a distant sample from a spacecraft. The robot can surround the object with the whole-body and bring it closer using the combined approach $\left(A_{4}\right)$. Then, the configuration can be changed using the time-based motion strategy $\left(A_{1}\right)$ in order to release it.

For example, if a robot is located on a spacecraft, it can perform different tasks, relying on its workspace and the moving capabilities of the vehicle. However, artificial satellites or space stations are not designed to be moved all the time. Then, regular and non-extensible manipulators in these scenarios have limited functionalities. Nevertheless, growing robots can be deployed in compact spaces and then extended to reach remote locations. Thus, their workspace can become larger and less dependent on the movements of the spacecraft. For instance, if a remote object needs to be grasped, maybe a regular hyper-redundant robot cannot reach it (Figure 5.37a), but a growing robot could (Figure 5.37b).

The extension process of many growing robots is irreversible, hindering their retraction back to the base. Then, a combined approach $\left(A_{4}\right)$ can be used here to control a new movement for the robot. For example, the combination of the surrounding and folding behaviours $\left(B_{11}\right.$ and $\left.\bar{B}_{17}\right)$ lead to a very interesting behaviour. It keeps the object grasped while, at the same time, folding the robot body approaching to the base (Figure 5.37c). This strategy is non-guided so the robot does not need to know the exact position, size or morphology of the object. It successfully manages to grasp the object as long as it is enclosed in the area of action.

Once the object is grasped, the time-based motion strategy $\left(A_{1}\right)$ lets the object be released by changing the shape of the robot. Then, the straightening behaviour $\left(\bar{B}_{11}\right)$ can be applied to the whole-body, aligning all the control points of the robot (Figure 5.37d). 
a

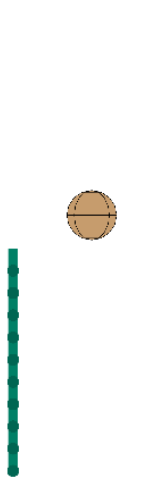

c

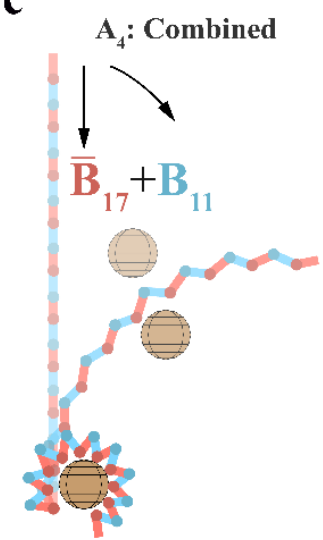

b

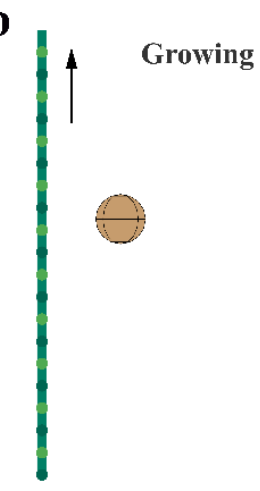

d

$A_{1}$ : Time-based

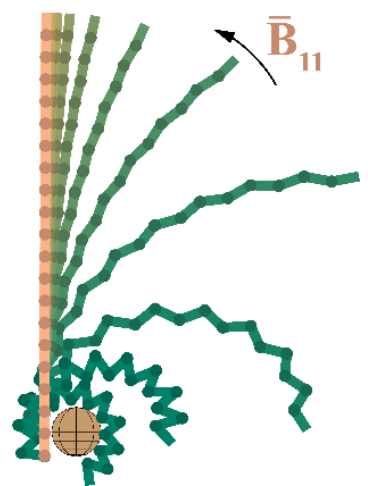

Figure 5.37. (a) A regular hyper-redundant robot cannot reach very distant objects. (b) But growing robots can extend their length for that purpose. (c) The combined motion strategy $\left(A_{4}\right)$ can be used to fuse the surrounding and folding behaviours $\left(B_{11}\right.$ and $\left.\bar{B}_{17}\right)$. It can be useful to grab and bring an object closer. (d) Then, the time-based approach $\left(A_{1}\right)$ let the configuration change using the straightening behaviour $\left(\bar{B}_{11}\right)$ to release the object when desired.

As it can be seen, the behaviours explained in Section 5.2.1 can be used in multiple scenarios. But the use of different motion strategies presented in Section 5.2.2 exponentially increase the number of potential applications for hyper-redundant and growing robots. Moreover, this type of distributed control is based on simple and fundamental principles while, at the same time, let the completion of complex tasks in a flexible and elegant way. 


\subsection{Applications}

Once the robot Mach was fully designed and built (Section 3.1), it was possible to study how to manage its movements. It is possible to distinguish three different types of control. First, low-level control regulates electrical inputs to obtain a desired output in the actuators. Then, mid-level control supervises how to transform the values of the actuators to rotations in the joints. Finally, high-level control is proposed to manage the rotations of the joints to useful movements for the whole kinematic chain.

\subsubsection{Actuation control}

The robot is actuated by 21 motors, but the actuation box is expandable to use 3 additional ones for endeffector tools. Each of them has an encoder that is used for feedback and regulated by drivers (Figure 3.4a). The initial step to control the motors is the identification. It helps to understand its behaviour against variations in the inputs in order to adjust the outputs as desired. For that purpose, a motor load was applied to analyse a step response and obtain the correspondent transfer function (Eq. 5.28). As a result, it was analysed and could be either approximated by a first or a second-order function. An underdamped secondorder response was finally chosen to provide a better fit (Figure 5.38a).

$$
G(s)=\frac{95900}{(s+65.48)(s+43.02))}
$$

Eq. 5.28

Once the behaviour of the motor is modelled, a closed-loop control scheme is developed (Figure 5.38b). The input of the system is the desired position trajectory for each motor. Such input will be previously limited in velocity and acceleration according to the functioning capabilities of the motors. The output of the system will be the motor velocity that leads to the desired position. A cascaded proportional-integral-derivative (PID) controller is employed to close the loop: one loop for the velocity and another for the position. Additionally, a feed-forward control is applied to the input of the PID controller for the velocity, looking for avoiding undesirable delays in the response. This PID was tuned and provided with an anti-windup filter. On the other hand, the PID controller for the position was simpler since the integral and derivative actions could be neglected. 
A
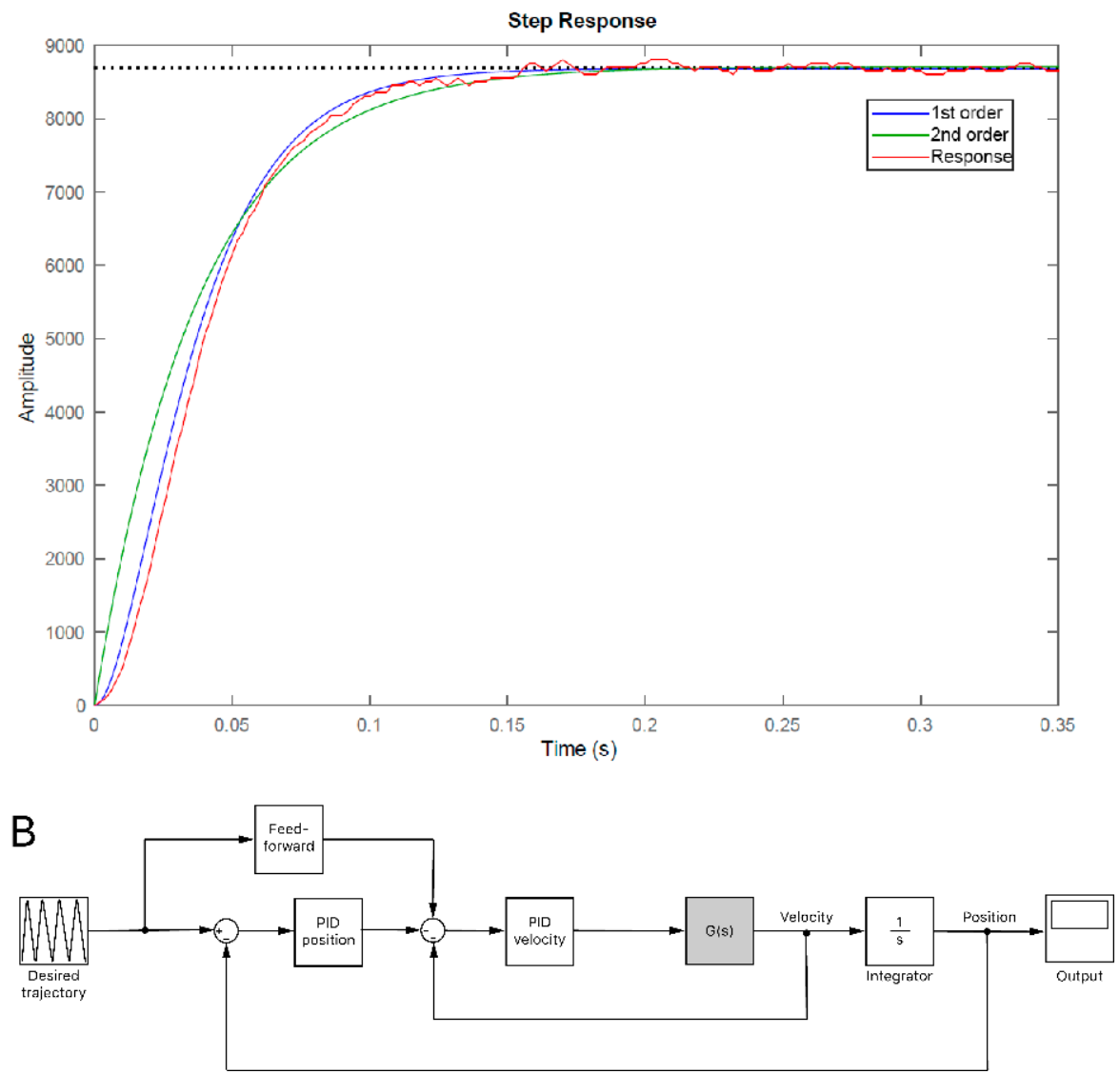

Figure 5.38. (a) Step response for the motor identification can fit to a first or a second-order system. (b) Control scheme for the motors. Two feedback controllers are used both for velocity and position and a feed-forward scheme is implemented to correct undesirable delays in the response.

\subsubsection{Joint control}

Once the motors are appropriately controlled, it is possible to obtain a reliable function that relates the linear displacements of cables to the rotations of joints. First, the robot is calibrated in a zero-angle pose and then, a reference system is created to measure the rotations and displacements. Two angles define each joint rotation: $\alpha$ represents the direction to rotate the joint, whereas $\beta$ is dependent on $\alpha$ and represents the magnitude of the rotation (Figure 5.39a). Thus, the resulting rotation $(R)$ could be defined using the Rodrigues rotation formula (Eq. 5.29), where I is the identity matrix and $K$ the cross-product matrix (Eq. 5.30).

$$
\begin{gathered}
R(\alpha, \beta)=I+\sin (\beta) K \alpha+(1-\cos (\beta))\left(K \alpha^{2}\right) \\
K=\left[\begin{array}{ccc}
0 & -k_{z} & k_{y} \\
k_{z} & 0 & -k_{x} \\
-k_{y} & k_{x} & 0
\end{array}\right] \text { and }\left[k_{x}, k_{y}, k_{z}\right]=\left[\cos \left(90^{\circ}+\alpha\right), \sin \left(90^{\circ}+\alpha\right), 0\right]
\end{gathered}
$$

Each module is driven using 3 equidistant cables at $120^{\circ}$ from each other. The displacements of each cable when the joint rotates can be obtained applying homogeneous matrix transformations (Eq. 5.31). The position of each cable $P$ after a rotation will lead to $P^{\prime}$ applying a translation, $T$ (Eq. 5.32), then the desired rotation, $R$ (Eq. 5.29), and finally the same translation again, $T$, as depicted in Figure 5.39b. 


$$
\begin{gathered}
P^{\prime}=\text { TRTP } \\
T=\left[\begin{array}{cccc}
1 & 0 & 0 & 0 \\
0 & 1 & 0 & 0 \\
0 & 0 & 1 & l / 2 \\
0 & 0 & 0 & 1
\end{array}\right]
\end{gathered}
$$

Eq. 5.32

It is important to highlight that, theoretically, the relation between the angle $\beta$ and the increment in the length of the cables is not perfectly linear (Figure 5.39C.1). However, the linear model will be used because the errors obtained from this assumption are negligible (Figure 5.39C.2).

Finally, trapezoidal speed-time curves were managed to generate coordinated trajectories. For this purpose, acceleration and deceleration speeds are established as well as the maximum global speed (08000 pulses-per-second). Then, the speed of all the motors is intentionally slowed down, so their movements begin and end in sync with the motor that needs to rotate the most.

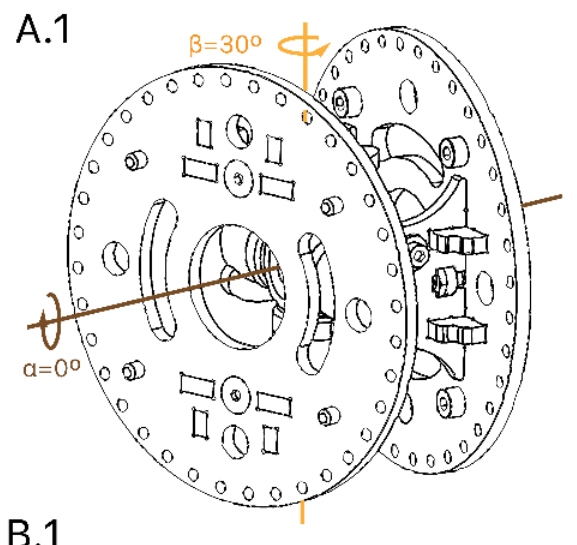

B.1

B.2

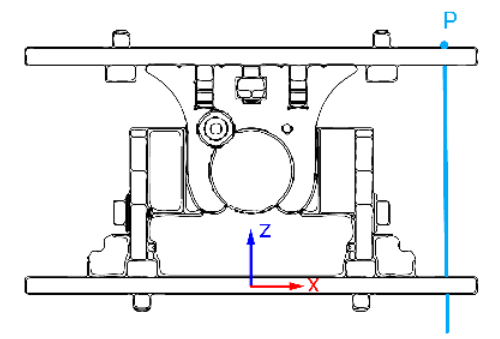

C.1

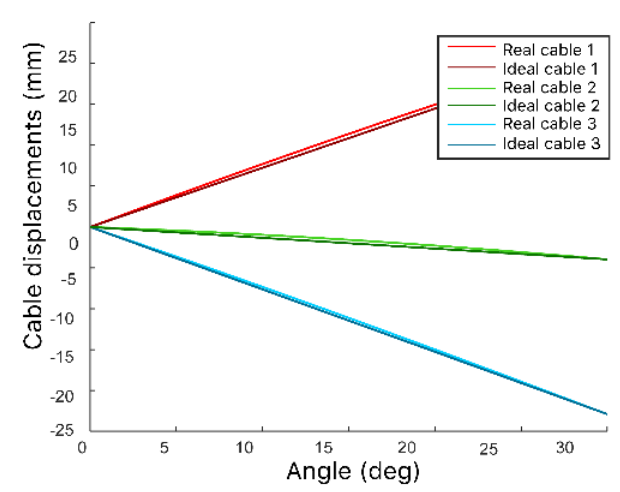

C. 2

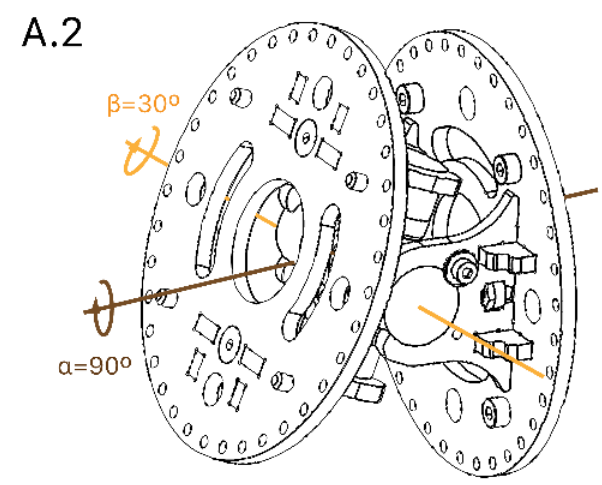

A.2
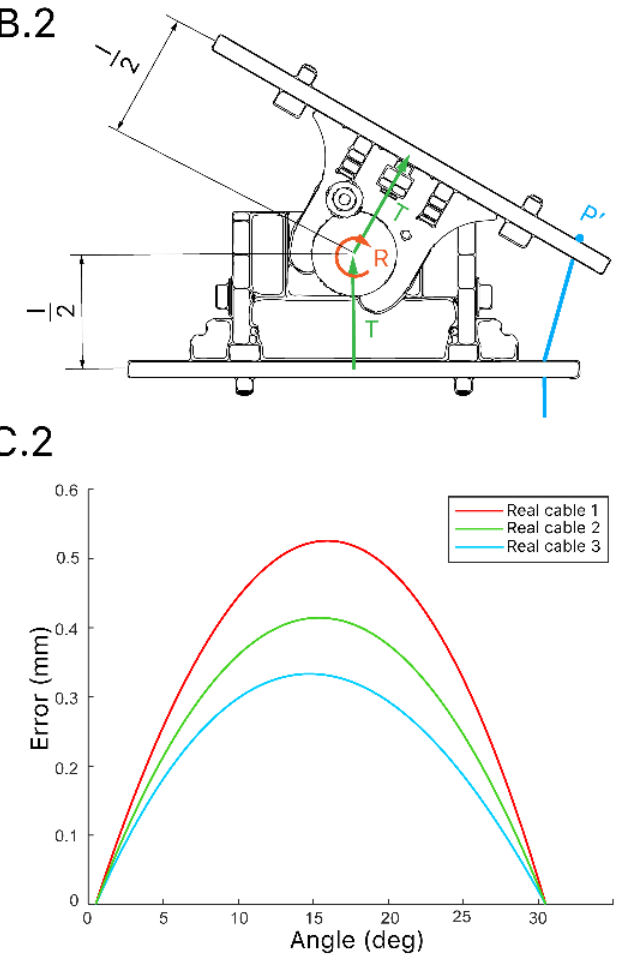

Figure 5.39. (a) The rotations are defined by two angles $\alpha$ and $\beta$ which do not directly represent the rotation axes, but the direction and the magnitude of the rotation, respectively. (b) Transformation of the pull point $P$ to $P^{\prime}$ after a joint rotation. (c.1) Difference between the real displacements of the cables of the same module versus the angle magnitude. (c.2) Error between the real displacements and the linear assumption. 


\subsubsection{Natural-CCD and shape control}

Once the control of joints is adequately managed, high-level controls are intended to manage the movements of the whole kinematic chain. Thus, such strategies will transform the independent joint movements in global ones for the robot body in order to perform specific applications.

It is possible to cite some of the most common control practices in this field. For example, the inverse kinematics aims to find the joint values that lead to a position and orientation for the end-effector. This problem is relatively easy to be solved for non-redundant robots. However, hyper-redundant ones offer a set of infinite possible solutions for each location of the end-effector. Therefore, selecting the most adequate is not always a simple task.

However, methods aiming to solve the inverse kinematics problem do not usually exploit all the kinematic capabilities for such robots. Thus, the shape-control appears to manage the whole configuration of the robot relying on a multi-variable reference [13]. The main challenge for this control is finding a good method to efficiently command such references in the three-dimensional space. Traditional methods were complex to design or not fully adapted to the needs of this kind of robots. Immersive technologies emerge as the best solution to teleoperate hyper-redundant robots for teleoperation applications [15]. This teleoperation scheme will be further analysed in Section 6.6.

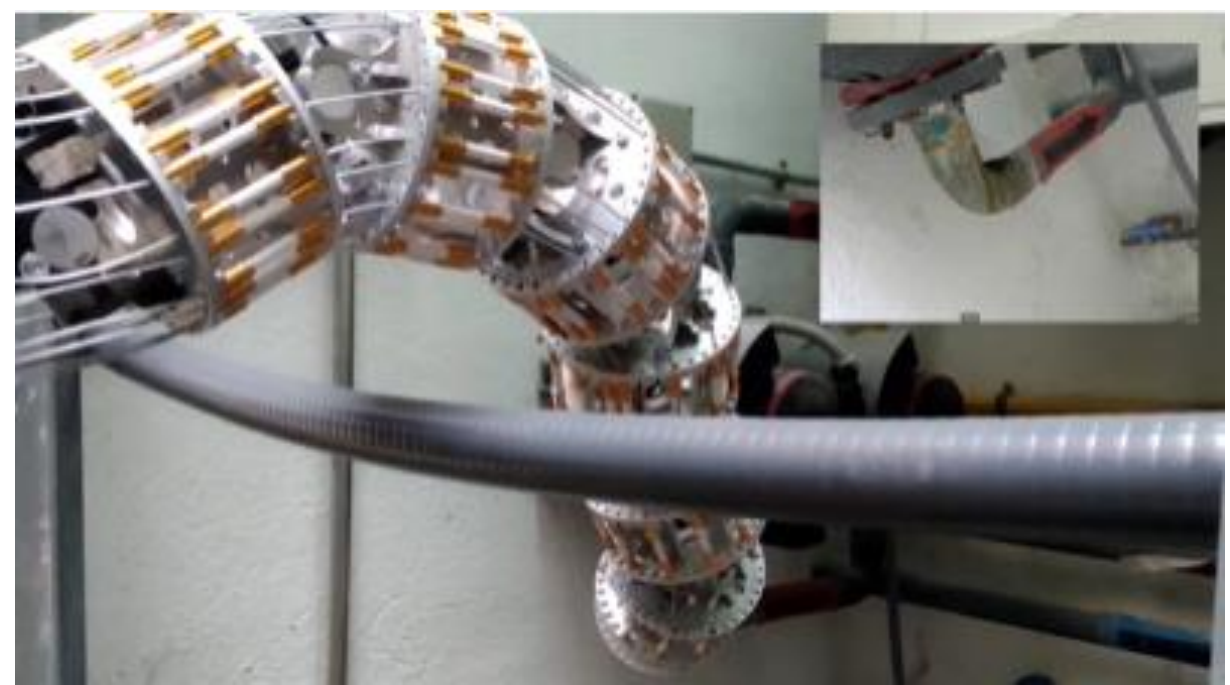

Figure 5.40. Shape control applied with a teleoperation scheme based on Mixed Reality 


\subsection{Discussion}

In this document, soft and hyper-redundant robots have been studied because of their potential benefits against conventional manipulators. They can perform better obstacle avoidance, are error-tolerant and offer mechanic and kinematic advantages. But one of the main challenges of this particular kind of robots is to effectively control them.

This chapter started proposing an algorithm to efficiently solve the inverse kinematics, since there exist an infinite number of possible solutions that satisfy that problem. This method is based on the Cyclic Coordinate Descent (CCD) and is entitled as Natural-CCD. This algorithm shows coherent and harmonious solutions as a result of graceful, incremental and iterative movements in the robot body. Using this algorithm, a robot with a sufficiently redundant number of equal-length links was found to lead to a particular end-effector path that is very present in nature: the Fibonacci spiral.

Moreover, this method presents multiple customization options to model hyper-redundant and continuous manipulators in a very clean and friendly interface. Some of them include the robot dimensions, initial orientation, number of modules and degrees-of-freedom per module, both prismatic and rotational ones, with maximum bending angles and fault-tolerance options, management of self and external collisions, development of cooperative hyper-redundant robots and even the possibility to add growing, branching and arborescent capabilities.

On the other hand, multiple control methods were designed, not just the inverse kinematics, but also shape controls, path following or follow-the-leader strategies among many others showing high-end performances. In fact, while previous works typically showed high computational times that grow exponentially against the number of degrees-of-freedom, the Natural-CCD presents far lower ones that allow real-time applications. But then, the solution to these control methods were found to be just the tip of the iceberg.

The Natural-CCD algorithm cyclically and uniformly moves all the degrees-of-freedom by small increments by using 3 relevant positions to obtain the desired rotation at every control point in the kinematic chain. Interestingly, multiple other emerging behaviours appear when those three variables are modified and assigned to different combinations of relevant positions in the robot. Some of the emerging behaviours include attraction, repulsion, surrounding, straightening, folding or circumnutations. Therefore, the solution to solve the inverse kinematics or the shape control are just 2 out of, at least, 40 of them.

Such behaviours have been classified in two groups. They were either called guided, whether an external reference exists, or non-guided when passively perform a specific action relying on their current shape. Regarding the attraction behaviours, they let the robot approach one or multiple references. In fact, solving the inverse kinematics problem can be understood as an end-effector attraction. However, similar behaviours let the robot be locally attracted to multiple references, enabling shape controls or even an oscillating-wave control. This second behaviour was obtained when the references were sequentially coupled. Interestingly, the frequency of the oscillating-wave could be dynamically controlled by growing robots as their body extends. This feature could be very useful to break through unstructured environments, enveloping the robot pose towards remote trajectories or performing follow-the-leader controls.

Similarly, repulsion behaviours let the robot be pushed away from objects. The local repulsion was particularly efficient to avoid obstacles by moving only nearby control points in the kinematic chain, without significantly affecting the rest of the configuration. Thus, this behaviour could be used to solve the motion planning of hyper-redundant robots. Accordingly, growing robots showed more flexibility in this matter since they could extend their bodies conforming with the environment. 
Besides, the surrounding and straightening behaviours were useful to grasp and release objects using the robot body as well as to enable local bracing. Interestingly, using non-guided surrounding behaviours, the computation is transferred to the morphology, so it is not necessary to know the exact position, size or morphology of the object to grasp. Growing robots could also take advantage of these behaviours by reaching farther objects or adjusting their shape to tight around irregular morphologies.

Additionally, the folding behaviour let hyper-redundant robots be contracted by following a zigzag pattern. This configuration makes the robot more compact and let it accumulate elastic energy that can be released for high-dynamic tasks.

Finally, the last behaviours studied in depth are circumnutations, described as a successive helical movement widespread among plants. Applications of this behaviour vary from attaching the robot for local bracing to grasping objects. Growing robots could take advantage of this behaviour by dynamically extending their length in order to control the magnitude, period or shape of such movements.

It is important to highlight that the appearance of these behaviours is not consequence of complex assumptions. In fact, it is based on slight modifications of three positions, the minimum number of variables to describe any rotation in the three-dimensional space. The behaviours emerge based on these local rules.

This work also proposed how to use such behaviours in 4 different motion strategies; firstly, time-based approaches switch different behaviours over time; secondly, section-based approaches apply different behaviours to different sections of the robot; thirdly, event-based approaches are applied based on environmental events; and lastly, the combined approach alternate different behaviours in the kinematic chain to create new ones. Even different combinations of these 4 main approaches could be simultaneously applied to complete complex tasks. Such motion strategies exponentially increase the flexibility and the number of potential applications for hyper-redundant and growing robots.

It is also important to acknowledge that this work has only analyzed some of these behaviours in depth. Initial configurations were usually straight and the movements were generated using the Natural-CCD algorithm, which applies uniform and small increments in the kinematic chain. Therefore, new behaviours and movements may emerge if those variables are further explored or if different combinations of such behaviours are proposed. Some of these behaviours seem to exhibit chaotic motion under these circumstances, but they may be useful for certain applications still unexplored.

In summary, this work proposes a framework to unify the underlying kinematics of highly articulated structures. Therefore, they are expected to play an important role in future high-level control strategies for hyper-redundant and growing robots.

The presented framework was used and tested in a real hyper-redundant robot and in a real environment performing a real task. This robot, Mach, was controlled from the actuators to high-level strategies based on the management of the whole kinematic chain. It is important to highlight that a kinematic control is reliable enough when dynamic forces can be neglected and the robot performs with moderately high cycle times. In contrast to traditional robotics with the actuators distributed in the robot body, the selected remote actuation scheme forces to calculate an intermediate control step to link the output of the actuators to the joints. Relating to the latter, it is very important to appropriately choose the actuators as well as the speed reducer system because they will highly determine the dimensioning, payload, resolution, accuracy and speed of the robot. The robot used was intended to perform an inspection task in a boiler room by using a teleoperation scheme and immersive technologies as it will be further explained in Section 6.6. 


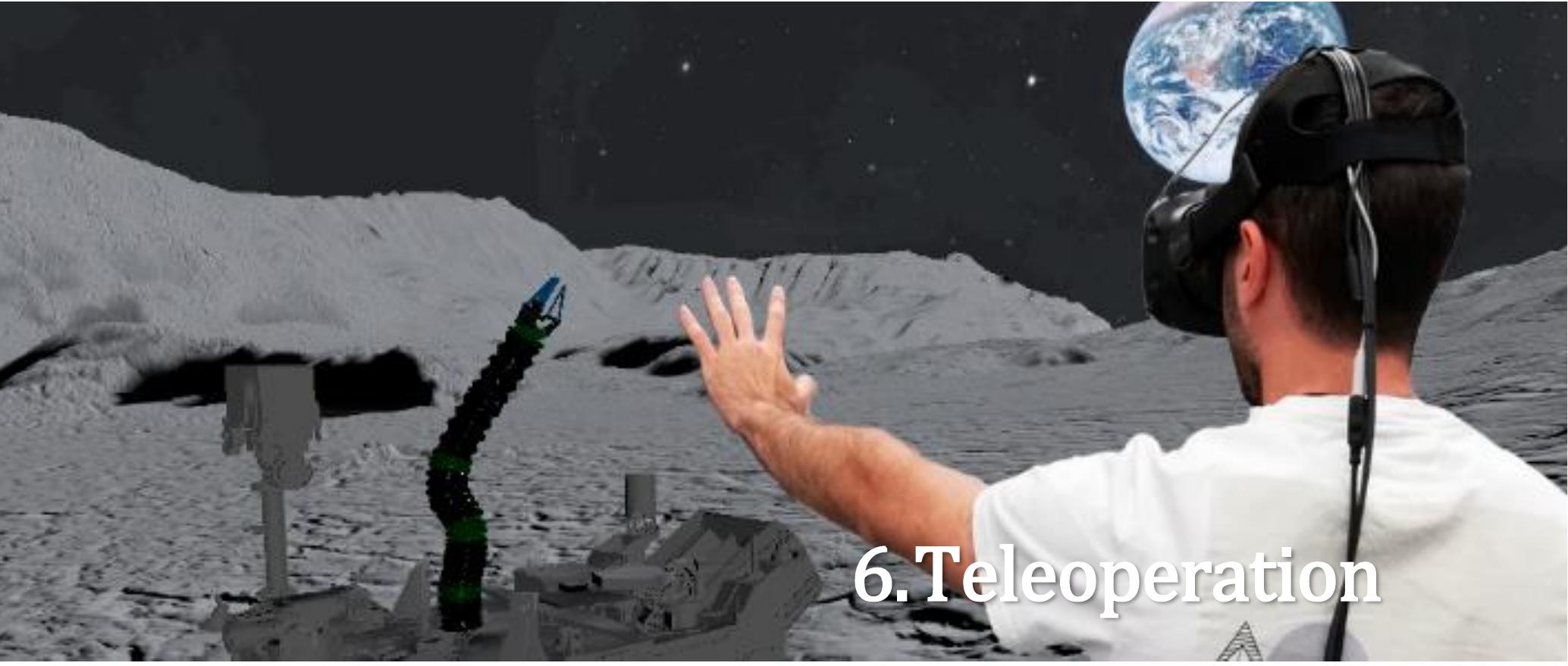

This section presents an analysis of immersive realities and natural language applied to the whole-body teleoperation of hyper-redundant robots. Such devices have a large number of degrees of freedom, so they often exhibit complex shapes frustrating their spatial understanding. This work aims to contrast two hypotheses; first, if immersive interfaces enhance the telepresence and efficiency against conventional ones; and second, if natural language reduces workload and improves performance against other conventional tools. A total of 2 interfaces and 6 interaction tools have been tested by a population of 50 people. As a result, immersive interfaces were more efficient, improved situational awareness and visual feedback, and were chosen by $94 \%$ of participants against conventional interfaces. On the other hand, participants performed better using natural language than conventional tools despite having less previous experience with the first ones. Additionally, according to $52 \%$ of the population, the preferred interaction tool was a mixed strategy that combined voice recognition and hand gestures. Therefore, it is concluded that immersive realities and natural language should play a very important role in the near future of hyper-redundant robots and their teleoperation.

Further information of this section can be found in an original previous publication ${ }^{16,17}$. Also, an explanatory of this video can be found in the Electronic Supplementary Material (Video S. 5).

\footnotetext{
${ }^{16}$ Andrés Martín-Barrio et al., Application of immersive technologies and natural language to hyper-redundant robot teleoperation. Virtual Reality, 2019, p. $1-15$.

${ }^{17}$ Andrés Martín-Barrio et al., Design of a Hyper-Redundant Robot and Teleoperation Using Mixed Reality for Inspection Tasks. Sensors 20.8, 2020: p. 2181
} 


\subsection{Context}

Traditionally, highly-articulated robots are controlled from the inverse kinematics perspective. In other words, controlling just the tip of the robot. However, commanding the end-effector location of a hyperredundant robot offers a limited number of applications. Besides, methods aiming to solve the inverse kinematics problem usually do not exploit all the kinematic capabilities for such robots. Thus, shape controls emerge as other type of control to manage the whole-body. This work will focus on the application of this type of control scheme. Under this paradigm, the entire configuration of the robot is controlled in order to perform tasks such as local avoidance of obstacles, surrounding objects or follow-the-leader guidance. Therefore, one reference would not be longer needed to control such robots, but multiple ones instead.

Strategies to adequately define this set of references are usually studied in telerobotics. This outlook implies linking a human operator and a robot in order to execute a remote task. Thus, whole-body teleoperation of a hyper-redundant robot should study how to properly associate a set of user-defined references to shape its pose. This field is highly related to telepresence, which means that the information about the remote environment is naturally displayed to the operator, implying a feeling of presence at the remote site. A good degree of telepresence guarantees the feasibility of a determined manipulation task [14]. Since hyperredundant robots often exhibit intricate shapes, hindering their spatial understanding, maybe emerging immersive technologies can be a good solution to solve this issue.

According to the previous definition, immersive realities could be understood as those real or simulated environments in which a perceiver experiences telepresence [16, 17]. For example, Virtual Reality (VR) provides interaction of virtual elements in virtual environments, Augmented Reality (AR) features interaction of virtual elements in real environments, and Mixed Reality(MR) offers interaction of real and virtual elements through real or virtual environments [18]. Nowadays, all these realities are achieved using headsets, headphones, glasses or controllers among several other devices, providing stimulus to the human senses including, but not limited to, vision, audition and touch. This feedback can be used within a teleoperation system to properly manage the pose of hyper-redundant robots.

On the other hand, traditional telerobotic systems have often relied on a master, intended to command the movements of a remote robot, called slave. These master-slave architectures were conceived in this manner to be transparent, in other words, to exactly reproduce the remote environment [14]. However, they often implied the design and construction of a physical interface to reproduce the real-robot movements. Immersive technologies could replace those physical masters within the virtual world while enhancing the immersion and telepresence. But such transparency will highly rely on the interaction tools used to replace those physical architectures. In that context, natural language could be used as one possible tool. It is understood as a rich, intuitive mechanism by which humans can interact with systems around them, offering sufficient signal to support robot task planning [264].

Accordingly, this section aims to contrast two hypotheses; the first one states that immersive interfaces are better in terms of efficiency and situational awareness against conventional ones to teleoperate hyperredundant robots; the second one states that natural language can improve the performance and reduce the workload against conventional tools for the same purpose.

The first main contribution of this work is the comparison of emergent immersive technologies against conventional ones to teleoperate the whole-body of hyper-redundant manipulators. Furthermore, an analysis of natural language and other tools used to interact within the immersive scenarios has been conducted and compared against conventional tools. Therefore, this section is expected to clear the path to which the research of teleoperation of hyper-redundant, continuous and soft robots should be focused on. 


\subsection{System setup}

The system arrangement will rely on the information exchanged between the user and the robot in both directions. Thus, communication is named bilateral (Figure 6.1). In other words, the user sends information to operate the robot in one way, whereas the robot transmits information to the user in the other way in order to report its current pose, both in real-time. As a reminder, a real-time system can be understood as an information processing activity that responds to an externally generated stimulus in a previously determined frame of time [245]. In the presented system, this bilateral communication performs a cycle with a frequency of $10 \mathrm{~Hz}$, which depending on the application can be acceptable or not. In this work, it was high enough to test the capabilities of the interfaces that will be explained in Section 6.3.

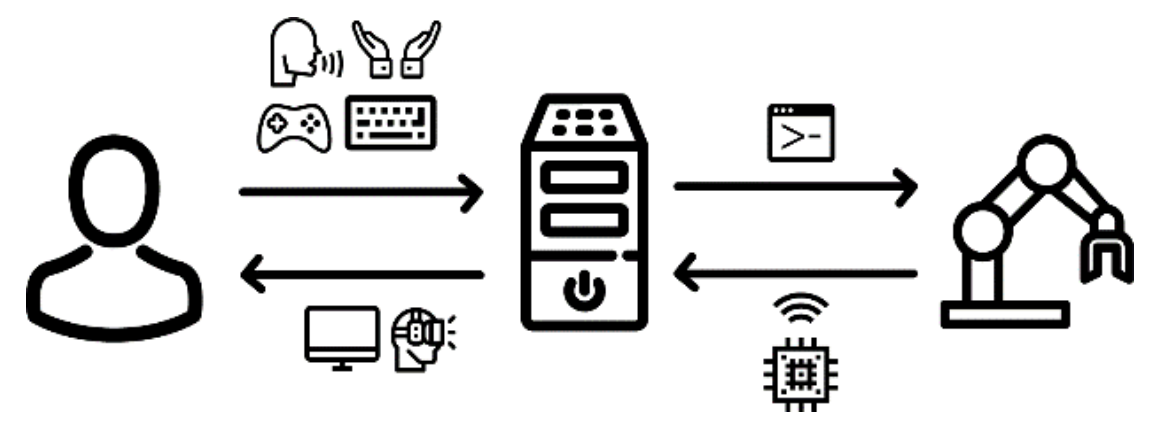

Figure 6.1. Bilateral scheme for the robot teleoperation.

The bilateral controller implemented in this work follows a position-position scheme. Thus, the robot will move to a determined position previously commanded by the user. Although this scheme is very simple, it is the best suited when the master-slave size relation is similar, there are no limitations in the slave speed, a high level of telepresence is required and the task involves a high number of degrees of freedom [265]. All these requirements describe the developed resources and setup, so the position-position scheme will be used.

In this work, the hyper-redundant and soft manipulator Kyma will be used to conduct the experiments [266] (Figure 6.2). Its structure consists of a set of bellows that allow both flexion and prismatic movements. It is divided into 4 sections, each one actuated by 3 angularly-equidistant tendons and motors. Therefore, teleoperating this robot will rely on 4 input positions. Moreover, the robot is also equipped with a gripper for pick and place operations. 


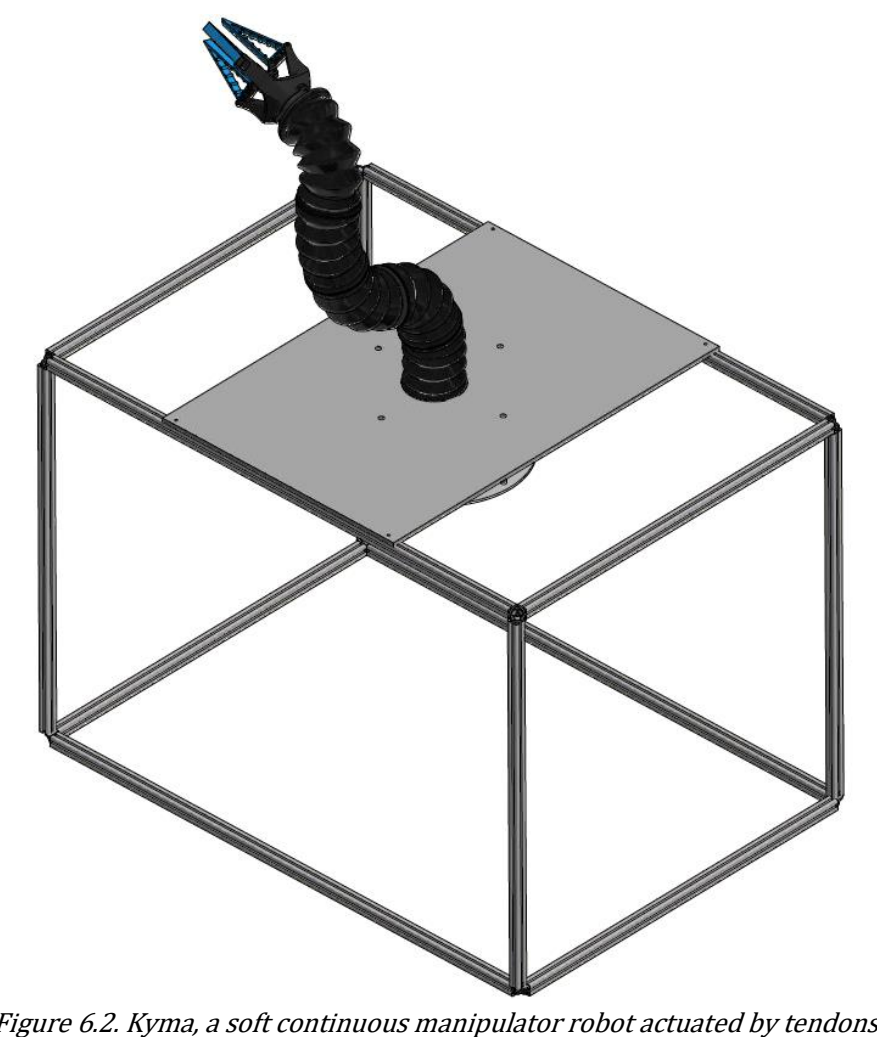

Additionally, a computer is used to host the interfaces and control the robot movements. It uses different peripherals to transmit or capture information from the user such as a display monitor, a VR headset, a keyboard or a gaming controller. The robot feedback to the user will be visual, so it will rely on displaying its whole-body configuration relying on its on-board sensory system based on Inertial Measurement Units (IMUs).

Therefore, the developed setup corresponds to Mixed Reality (MR), since interaction occurs with both real and virtual elements through real and virtual environments. This MR can be achieved from a Virtual Reality (VR) perspective, by introducing the user in a virtual world, or from an Augmented Reality (AR) one, by overlapping virtual elements over the real scene. In this work, the developments have been made in MR from both perspectives using the HTC Vive VR headset and the Hololens AR glasses respectively, although the experiments have been entirely conducted on the first ones.

It is worth highlighting that virtual and augmented environments are not competitors, but rather different perspectives for different purposes and applications. Usually, AR is thought to be useful in local environments, enriching the real world while VR is oriented to replicate remote or imaginary places by immersing the user in the environment. For example, teleoperating a robot in a nuclear reactor [267], or in a remote place such as the moon [268], are applications better suited for VR. However, teleoperating a robot in a surgery room [269] or in a cooperative environment for in-situ assemblies [270], are perspectives more appropriate for AR. 


\subsection{Human-robot interfaces}

This work aims to determine whether immersive interfaces are better or worse than conventional ones to teleoperate hyper-redundant robots. Hence, both interfaces have been created based on the same constitutive elements in Unity3D, a cross-platform real-time engine. A human-robot interface can be understood as a set of elements that enable the communication and interaction between a person and a robotic system [271]. In this context, a good interface would be clear, concise, familiar, responsive, consistent, aesthetic, efficient and forgiving. Binding such features will give place to an efficient interface while also providing high situational awareness.

Teleoperation of hyper-redundant robots, similarly to multi-robot systems, usually requires a relatively high number of inputs to appropriately manage their whole-body [272]. And it is important to highlight that human beings perform better paying full attention to one thing at one time [273]. Therefore, choosing the most convenient interface for this purpose is very important in order to maximize efficiency. In the context of teleoperating robots, efficiency will be defined as the ability to maximize precision and speed by commanding a robot to perform a determined task.

In this context, the number of inputs will be highly dependent on the number of degrees of freedom, but not always strictly the same. For example, several hyper-redundant devices such as continuous or soft robots can be considered as under-actuated [7]. Such systems have a higher number of degrees of freedom than actuators, so their teleoperation will usually rely on a model to estimate their pose when being teleoperated. In the case of Kyma (Figure 6.2), it has 4 sections so the number of inputs will be the same, although the virtual robot will rely on a Piecewise Constant Curvature (PCC) model to reconstruct its whole body [162]. This is the main difference between commanding the whole-body of a non-redundant robot and the shape of a continuous or soft one.

On the other hand, situational awareness can be defined as the perception of elements in the environment within a volume of time and space, the comprehension of their meaning, and the projection of their status in the near future [274]. Under these circumstances, a high level of situational awareness is considered a result of a high level of telepresence. Thus, situational awareness will be measured later to infer the quality of telepresence in the created interfaces.

In any of these two human-robot interfaces, the scenes are three-dimensional, although in the conventional interface the information is shown in a two-dimensional (2D) display. The user is sitting looking at the monitor and can use the keyboard to move in 3D a sphere that symbolizes the position where the robot's end-effector is commanded to move. The user is expected to move the input towards 5 predefined positions and, in case of making a selection, the robot will move accordingly and its pose will be updated in the interface in real-time (Figure 6.3). 


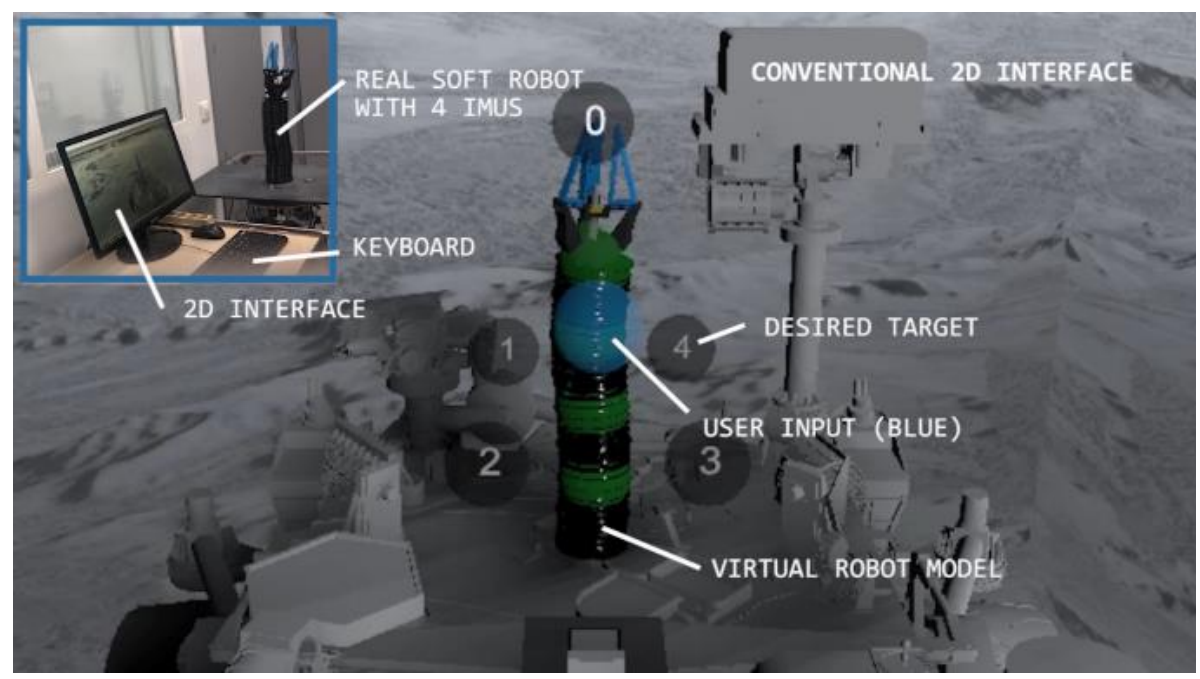

Figure 6.3. Conventional interface. Here the scene is displayed in two dimensions by a monitor. The robot is commanded to move using a keyboard and its pose is displayed in the interface in real-time.

In the immersive interface, the same information is displayed, but in this case, an immersive headset with three-dimensional (3D) stereoscopic vision is used. In this section, only visual feedback will be further analysed to enlighten which one of such interfaces is better suited to enhance telepresence and efficiency, and regardless of the interaction strategy used (Figure 6.4).

These tools will be analysed in Section 6.4, whereas specific details of the experiments and results are explained in Section 6.5.

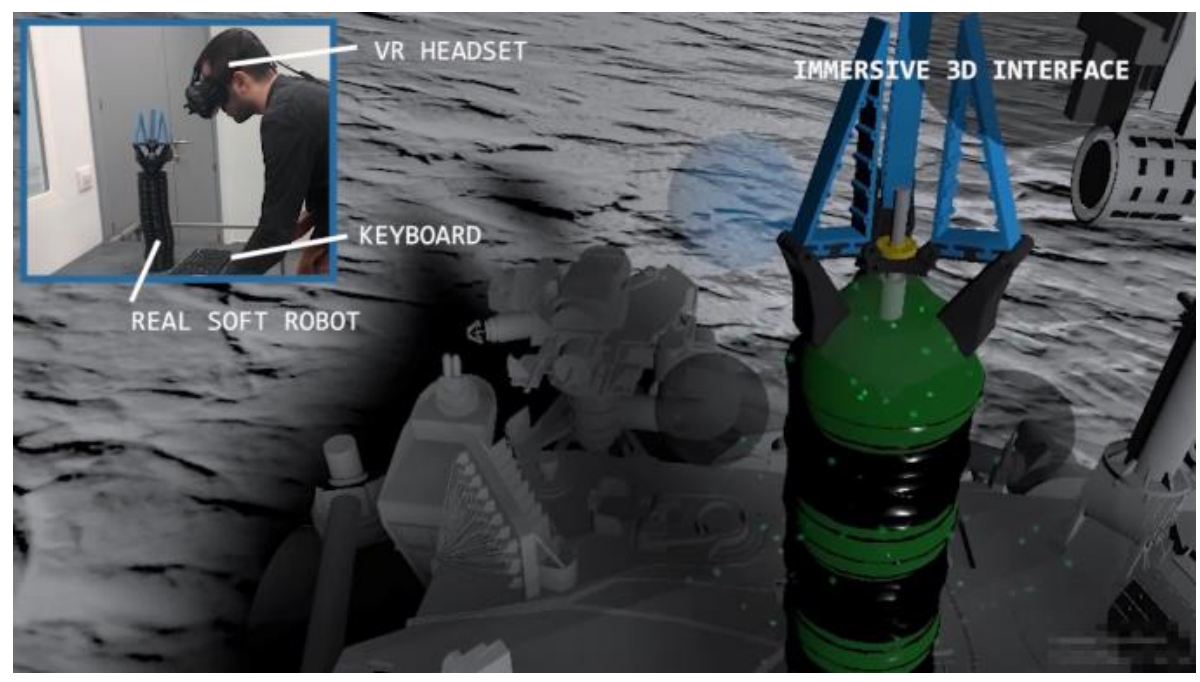

Figure 6.4. Immersive interface. The setup is nearly the same than in the conventional interface but using a VR headset instead of a monitor. 


\subsection{Interaction tools}

Another main purpose of this work aims at determining which strategy is more appropriate to teleoperate hyper-redundant robots in an immersive interface in terms of efficiency and workload.

In this context, efficiency will be defined in the same way as the previous Section 6.3, as the ability to maximize precision and speed. On the other hand, the workload can be defined as a hypothetical construct that represents the cost incurred by a human operator to achieve a particular level of performance [275].

As a result, 6 different approaches have been developed in Unity3D, making use of a computer, the HTC Vive VR headset and, in some cases, Xbox or Leap Motion controllers. These strategies are the following: controller, master-slave, local gestures, remote gestures, voice commands and a hybrid approach of gestures and voice recognition.

The strategy based on a controller was chosen to illustrate one of many possible conventional tools based on a physical device to command the movements. The master-slave was inspired by past efforts based on physical replicas of the robot; but in this case, the replica was designed in the virtual world, so it is easily scalable, the teleoperation transparency is preserved, and the construction and maintenance costs are minimized. The local gestures were developed to be the most intuitive since relied on shaping the real-size robot with the hands. While remote gestures were also conceived to manipulate the robot with the hands, they promise better interactions when the robot is too big or too small. Additionally, voice commands were implemented to let the robot be moved when the operator hands are busy or their movement is hindered. Finally, the gestures and voice strategy combines two natural language approaches to determine if they synergize and give place to better results.

In every method, the user has to exploit all the available resources to adequately manage the pose of a virtual robot, which has to be approached towards a predefined virtual reference. This reference was only conceived to measure the performance and conduct the experiments. A more detailed description of each interaction tool is explained as follows. Basically, in all these interaction tools the user will be introduced in a Virtual Reality scenario and then, will be requested to move a virtual robot (green) towards a virtual reference (blue). As it will be explained in Section 6.5, it will be requested to complete the tasks as fast and precise as possible.

\subsubsection{Controller}

This strategy uses an Xbox gaming controller to teleoperate the robot, so it can be defined as a conventional technique against others developed in this work based on natural language. The user, provided with a $V R$ headset and the controller, will rely on pressing buttons and axis to move the virtual robot towards the reference.

First, the desired joint must be selected using some buttons and then, it can be moved along the Cartesian directions using the controller's axis. The implementation allows simultaneous inputs so the user can combine the three directions to perform 3D movements. The joints' speed can be adjusted by the quantity of displacement in the axis. In fact, these inputs have been filtered to minimize drifting or undesired sensibility issues (Figure 6.5). 


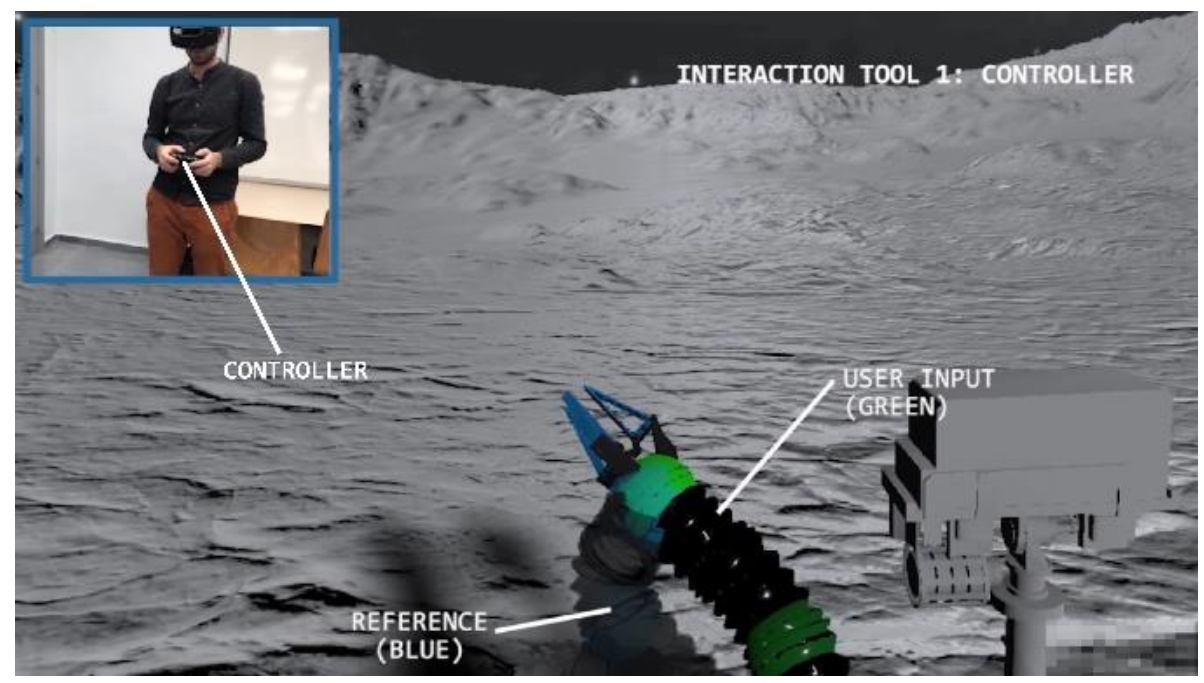

Figure 6.5. Controller strategy. In this interaction tool, a gaming controller is used to select and move the robot joints one by one using buttons and axis.

\subsubsection{Master-slave}

Traditionally, the master-slave architecture often implied the design and construction of one physical device (master) to reproduce the real-robot movements (slave). In this context, the system is more transparent if the similarity of the master and the slave is higher. However, creating a physical replica of the robot with on-board sensors usually involved additional cost, maintenance and construction time.

Currently, immersive realities offer the possibility of rapidly and easily creating a virtual master with the same features of the slave without any physical restriction on its construction, and any additional cost or maintenance. According to the specific literature, all the presented strategies in this chapter have a master and a slave. However, this strategy takes its name inspired in traditional master-slave physical models, since the robot relies on a virtual model of different size. Thus, this strategy could be most beneficial when the robot size is too big or too small to be adequately manipulated using regular gestures.

In this scenario, the user's hands were tracked using the Leap Motion controller, which uses a set of infrared light-emitting diodes (LED) and cameras to track the movements of hands and fingers. The sense of proprioception using this device is very high, so the users feel like touching virtual objects, although they are not.

Thus, when the user raises one hand, a smaller replica of the robot is created. Additionally, it will follow the hand movements until it closes so it can be positioned as desired. Then, the other hand can be raised to gently push the robot joints towards the desired reference by applying contact. By doing this, the slave will be moved the same way as the master is being manipulated. But in any case the user will enter in contact with the slave directly (Figure 6.6). 


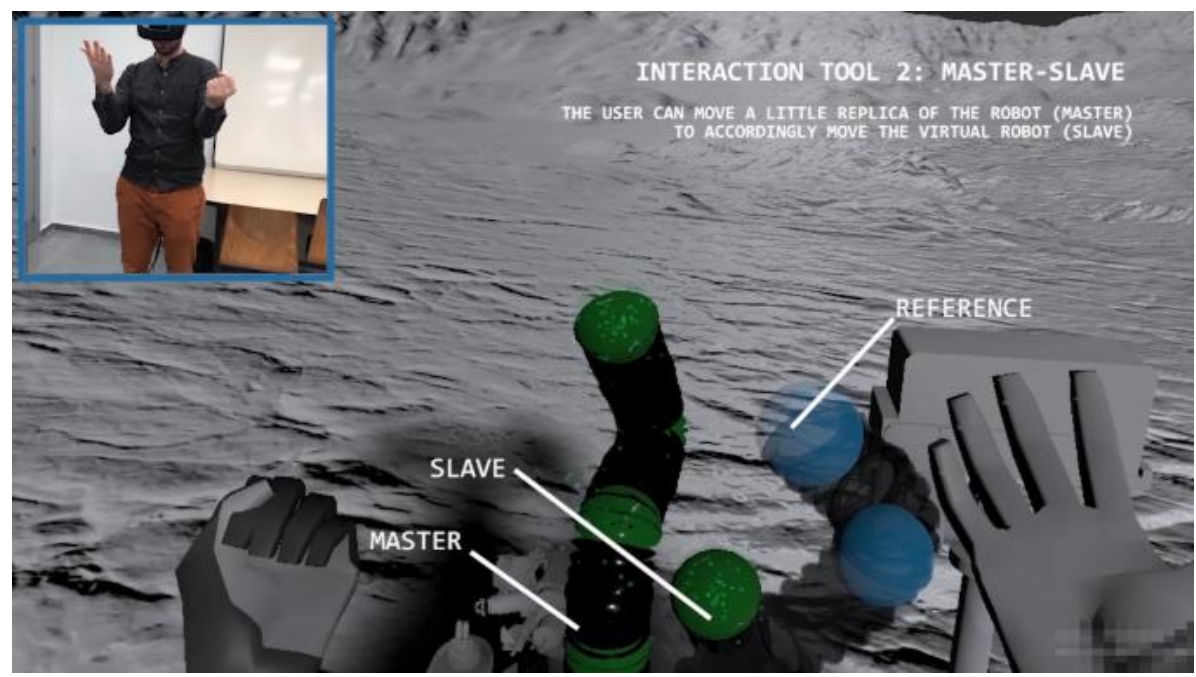

Figure 6.6. Master-slave strategy. Here, a little replica of the virtual robot (master) is manipulated so the bigger one (slave) is identically moved towards the desired reference.

\subsubsection{Local gestures}

This strategy also uses hand tracking to capture gestures. However, the main difference is that the user must approach the virtual real-size robot and use the hands to push the robot joints towards the desired reference by direct contact. It was found that pushing enhanced efficiency and reduced workload against grabbing, so the joints could not be grabbed but only pushed using this approach.

Here, the performance is highly dependent on the relative size of the robot and the user. If the virtual robot is too small, it will be difficult to be precise. On the other hand, if the virtual robot is too big, then the user will be very slow and will need to move more in the workspace. However, in $V R$ is possible to scale all the environment so the perfect size of the robot can be chosen to the most adequate even if the real one has other dimensions (Figure 6.7).

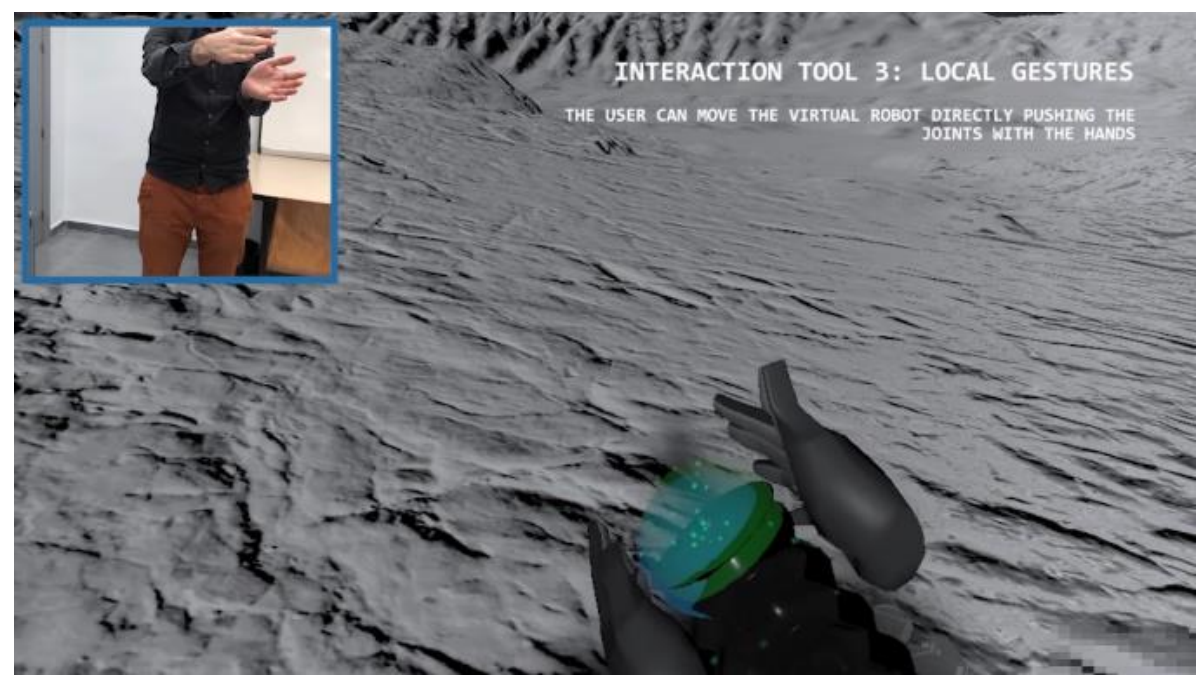

Figure 6.7. Local gestures strategy. The user's hands are tracked using a Leap Motion system so they can be used to directly interact with the virtual robot. 


\subsubsection{Remote gestures}

Next, similarly to the master-slave strategy, remote gestures allow the robot to be moved without direct contact with the virtual robot model. In this case, the virtual robot model is moved without any intermediary using hand gestures as well.

Therefore, the user can open the index finger on one hand, so a virtual laser appears as an extension to easily select the joint to move. Then, when the other hand is opened, it will move the selected joint accordingly to its own movement. When this hand closes, the joint does not move.

This implemented technique is called reindexing. It can be defined as the temporary removal of connection between master and slave when the range of motion is smaller for the first than for the second one [276]. It is the same principle as when a computer mouse is raised when gets closer to the edge of a table.

Since this strategy relies on remote gestures, the operator is not expected to move a lot, so reindexing is very important to minimize the difference of size between master and the slave (Figure 6.8).

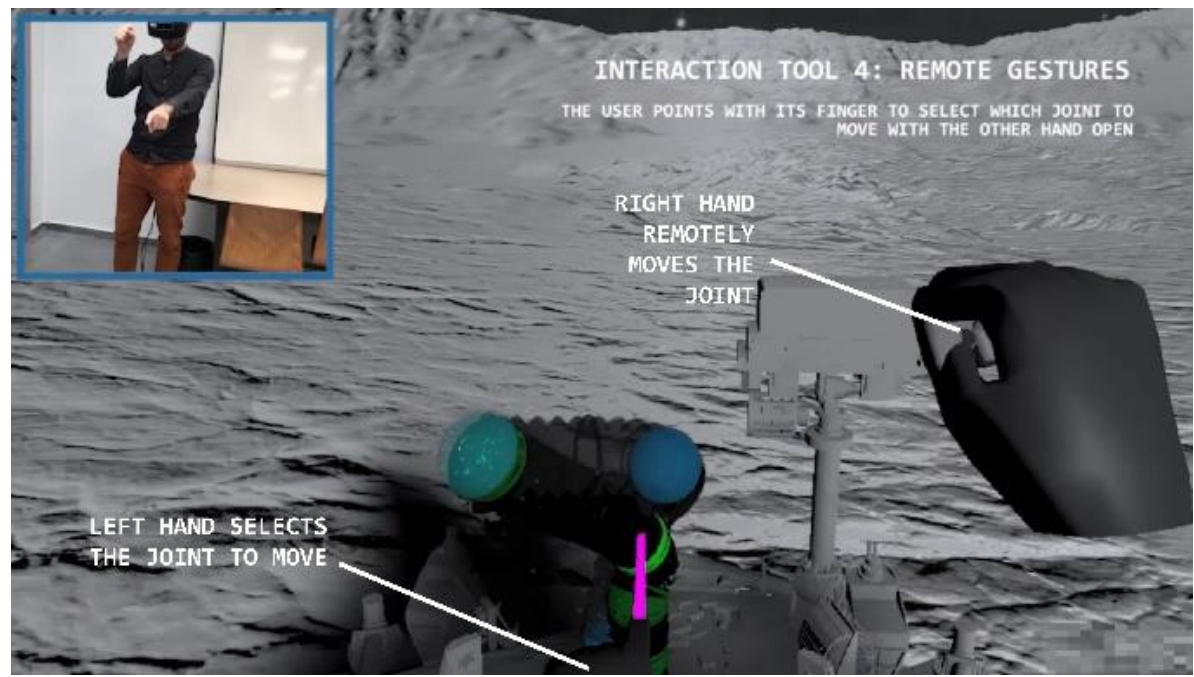

Figure 6.8. Remote gestures strategy. In this setup, the user can select the joint to move by pointing with a virtual laser that emanates from the left index finger. Then, the selected joint remotely moves when the right hand is opened.

\subsubsection{Voice commands}

This following tool is based on speech recognition to command the robot movements. This approach was created so the user can teleoperate the robot even if the hands are busy or their movement is hindered. Therefore, the user needed to remember some intuitive commands previously defined in a dictionary. Such commands could activate the movement of the robot by small increments or continuously by defining when to start and stop. This work opted for the second alternative because it was found to increase the precision and dramatically reduce the number of commands.

Therefore, the joint to move is first selected by pronouncing numbers, in this case, from 1 to 4 . Then the speed can be defined as slow, medium or fast. Only three levels of speed are taken to reduce the complexity of the strategy. However, they are tuned for the robot dimensions, so the slowest one is aimed to be more precise, while the fastest is more adequate to move long distances. Finally, the user can speak in which direction to move the selected joint among left, right, forward, backward, up and down. 
These directions have not been allowed to be combined using this method because the result was unfamiliar to the users. Alternatively, this method could be implemented using voice intensity as a variable to select the movement speed (Figure 6.9).

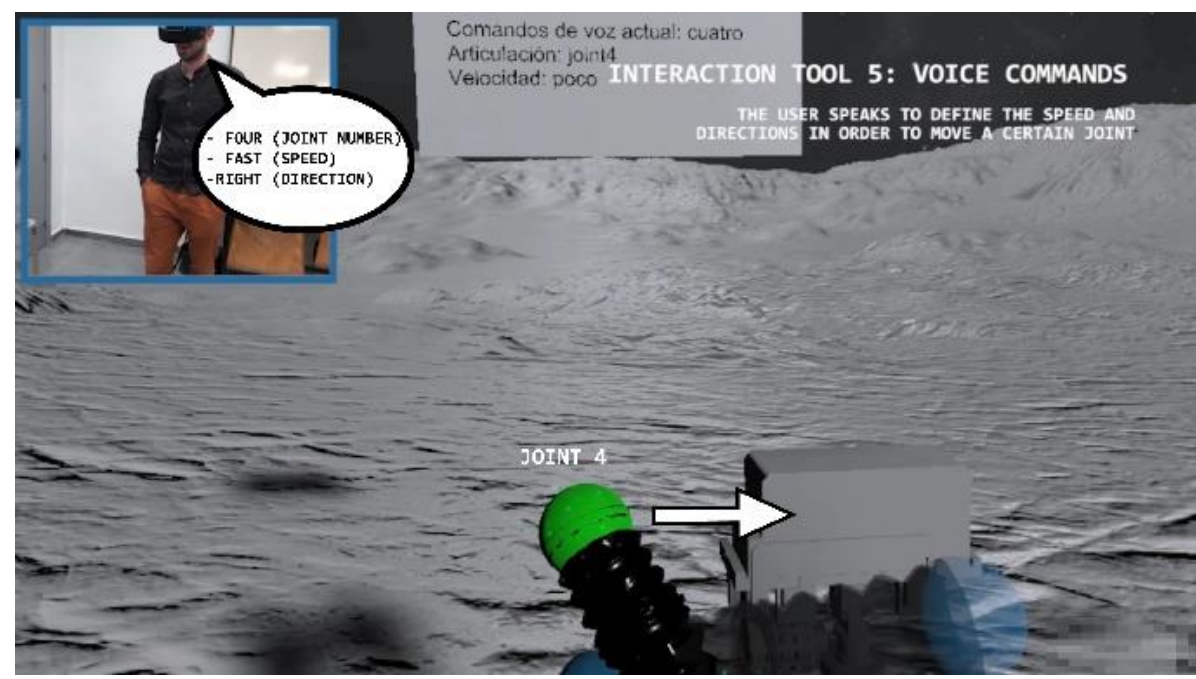

Figure 6.9. Voice commands strategy. The VR headset records the user's voice to recognize simple commands in order to select the joint to move, its speed and the direction.

\subsubsection{Gestures and voice}

If the user can speak and move in the real environment, another approach is based on using both voice and gestures to command the robot. Thus, it is the only multimodal approach in this work. Several methods could be proposed in this matter but, it was finally decided to combine what voice and gestures do better.

Therefore, and similarly to the previous method, Voice Commands are programmed to select the desired joint by pronouncing which joint to move from 1 to 4 . Then, similarly to the Remote Gestures strategy, the user can raise the hand to move the joint with reindexing, as explained before. Hence, the voice is proposed as an alternative of pointing with the other hand to unequivocally select the joints, and gestures are selected to efficiently move the joint. This method can be understood as a hybrid method within the natural-language strategies (Figure 6.10).

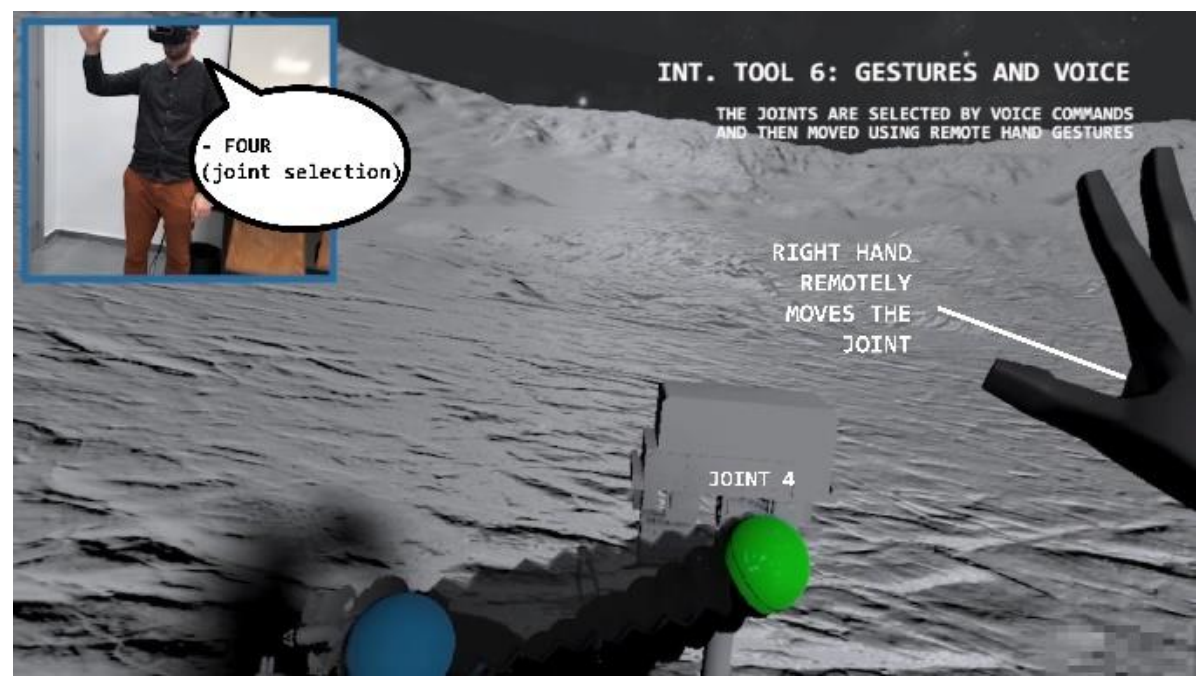

Figure 6.10. Gestures and voice strategy. This strategy uses the voice to select a joint and hand gestures to remotely move it. 


\subsection{Results and validation}

Two experiments were conducted between February and March of 2019 to validate the proposed hypotheses of this work. As a reminder, the first one wonders whether immersive 3D interfaces or conventional 2D ones are better suited to teleoperate hyper-redundant robots. On the other hand, the second one aims at selecting which interaction tools are more efficient and reduce workload for the same purpose.

For both experiments, a sample of $\mathbf{5 0}$ volunteers was selected in order to be heterogeneous enough to lead to generalizable results, while also homogeneous enough to minimize variances due to factors not relevant in the context of this work. For example, an engineering student with experience using computers or videogames is expected to perform differently compared to an elderly person. Since this work is not intended to compare people skills, but the interfaces and interaction tools instead, the population sample should exhibit a compromise between heterogeneity and homogeneity.

Therefore, the participants have different academic backgrounds but all of them come from a university context, they have different genders and ages but come from the same generation, and have different previous experience in immersive realities, robotics and videogames (Table 6.1).

\begin{tabular}{lll}
\hline Personal data & Mean & Variance \\
\hline Age (years) & 24.2 & 11.5 \\
VR experience (\%) & 35.6 & 58.4 \\
AR experience (\%) & 28.1 & 50.2 \\
Robotics experience (\%) & 50.1 & 79.2 \\
Videogame experience (\%) & 66.9 & 81.5 \\
\cline { 2 - 2 } Table 6.1. Mean and variance values of the participant ages and their subjective previous experience (the higher, the more \\
experience).
\end{tabular}

Since this sample is just one sector of the potential population, the obtained data is first evaluated to measure the goodness of fit to a determined statistical distribution. For this purpose, the KolmogorovSmirnov test is applied to all the normalized data using the software Matlab [277]. As a result, it has been found that the data follow a normal distribution. Based on this assumption, determining if the means of two sets of data are significantly different from each other, require the use of a Student's t-test or one-way analysis of variance (ANOVA), so they will be applied throughout this chapter. If not mentioned, the chosen significance level is $\alpha=0.05$.

\subsubsection{Human-robot interfaces}

In order to test the objective efficiency of the developed interfaces in terms of precision and time, the participants were asked to move a blue sphere as a representation to where the end-effector of the robot is desired to move. They were warned to do it equally as fast and precise as possible. This input could be moved towards 5 numbered and predefined target positions, depicted by grey spheres of the same size. The participants could practice for a while until they felt ready, in order to minimize the learning factor between interfaces. They could press a begin button and move the input towards 3 of the 5 target positions, previously specified. Then, a timer began to run, and the precision was measured as the mean of the Euclidean distances between the input and the desired targets. Both time and precision variables were first rescaled -min-max normalization- Eq. 6.1 among the data relative to both interfaces to allow further comparisons, then averaged Eq. 6.2), and then rescaled again Eq. 6.1. 


$$
\begin{gathered}
x^{\prime}=\frac{x-\min (x)}{\max (x)-\min (x)} \\
\bar{x}=\frac{1}{n} \sum_{i=1}^{n} x_{i}
\end{gathered}
$$

where $x$ is a single data for each participant, $x^{\prime}$ the scaled value relative to all the population for both interfaces, $\bar{x}$ the mean value between time and precision and $n$ the number of participants. By this method, speed and precision are measured the same to test efficiency since both parameters can be considered as equally important to teleoperate a generic hyper-redundant robot. However, it must be remarked that one variable could be more important than the other depending on the robot and its purpose.

(a) Efficiency

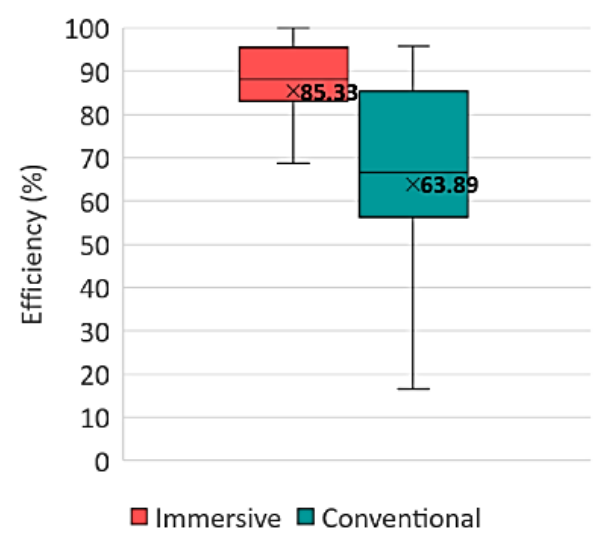

(c) Subjective visual feedback

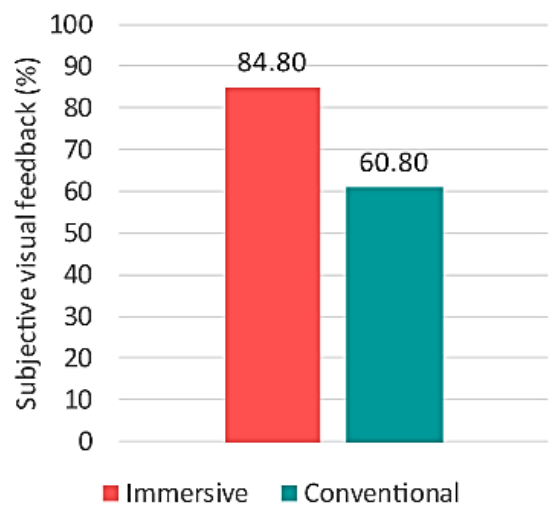

(b) Situational awareness

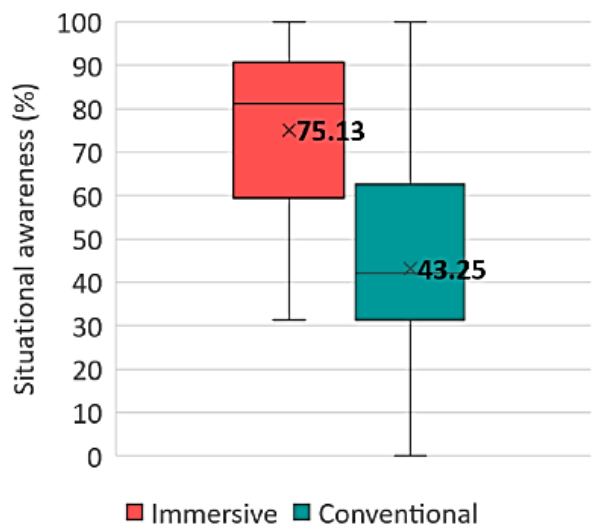

(d) Subjective preference

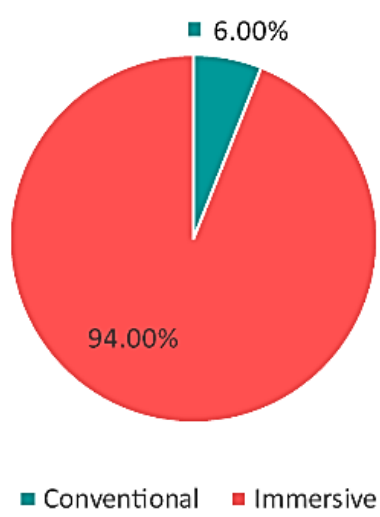

Figure 6.11. Results for the human-robot interfaces test. (a) Efficiency in terms of precision and time, (b) situational awareness, (c) subjective visual feedback and (d) subjective preference.

As a result, the efficiency is a $33.55 \%$ higher on average and the variance a $41.98 \%$ lower for the immersive interface than for the conventional one (Figure 6.11a). The results between immersive and conventional interfaces are significantly different between each other based on both the Student's $t$-test $\left(p=1.9 \cdot 10^{-6}\right)$ and the $A N O V A\left(p=6.6 \cdot 10^{-7}\right)$. Therefore, their comparison is statistically reliable. 
The situational awareness $\left(S_{A}\right)$ is measured in this document inspired by previous works [278]. Since such variable is subjective and depends on the environment setup, the questionnaire is usually custom-made. In this test, 3 questions were made to quantify the user perception of what was happening in the real world, compared to the images displayed on the interface. Therefore, the participants were able to see both the real robot and the interface in order to make an inference.

It is worth highlighting that the interface was programmed to hide the target number labels, so the participants were previously asked to remember them in order to test memory retention $\left(s_{1}\right)$. Moreover, they were asked to focus on which of the 3 commanded movements they thought their precision was higher $\left(s_{2}\right)$. To finish, they were asked to evaluate visual feedback from both interfaces $\left(s_{3}\right)$. Parameters $s_{1}$ and $s_{2}$ were binary variables which could take value 0 if the answer was wrong or 1 if it was right. Variable $S_{3}$, the visual feedback, it was measured from 1 to 10. All this variables were normalized Eq. 6.1 to allow further averaging Eq. 6.2). Furthermore, the influence of $s_{3}$ was increased to reduce the variance of the results a $10 \%$ Eq. 6.3.

$$
S_{A}=\frac{s_{1}+s_{2}+3 s_{3}}{5}
$$

Eq. 6.3

Afterwards, the resulting data is again rescaled Eq. 6.1 in order to facilitate comparisons between interfaces. The results show that situational awareness is a $73.71 \%$ higher in the immersive interface than for the conventional one (Figure 6.11b). Concerning the visual feedback evaluation, the participants were able to see the real robot and the model within the interface simultaneously and in real-time, so they could make reliable comparisons between them. As a result, they perceived that the visual feedback was a $39.47 \%$ higher for the immersive interface than for the conventional one on average (Figure 6.11c). These results are also significantly different based on the Student's $t$-test $\left(\mathrm{p}=1.8 \cdot 10^{-10}\right)$ and the ANOVA $\left(\mathrm{p}=4.3 \cdot 10^{-12}\right)$.

To finish, and regardless of previous questions, the participants were asked to subjectively choose between both interfaces assuming they had to perform the same task. Consequently, 94\% of the participants preferred to use the immersive interface (Figure 6.11d). Therefore, both subjective and objective parameters agree to select the immersive 3D interface to teleoperate hyper-redundant robots as the best to enhance efficiency and telepresence.

\subsubsection{Interaction tools}

The human-robot interfaces test shows that immersive ones obtain better results to teleoperate hyperredundant robots. The next inquiry is based on selecting which interaction strategy is better within this immersive reality for the same purpose. As a reminder, there are 6 developed strategies, as explained in Section 6.4: controller (CR), master-slave (MS), local gestures (LG), remote gestures (RG), voice commands (VC) and gestures and voice (GV). In this test, the participants used a VR headset to command the movements of a hypothetical virtual robot towards a randomly-defined set of references by using the previously mentioned interaction approaches.

On the one hand, the efficiency was similarly measured using both elapsed time and precision. For each one of the approaches, the user could also practice before the timer began in order to reduce the learning factor. And the precision was measured and averaged as the Euclidean distance between every 4 sections of the virtual robot and the relative ones of the reference, differently coloured to ease comprehension. 
In all the strategies, the user had to repeat the process 3 times, moving the virtual robot towards different references in order to increase the data normalization. Such references were randomly defined but bounded to a predefined and limited space so they would not differ too much from one participant to another. Therefore, the virtual robot joints were moved 3600 times in total in these experiments. Accordingly, the learning factor was minimized, whereas the randomness and repetitions standardize the data in order to achieve generalization.

Similarly to the previous experiment, the speed and time were first rescaled Eq. 6.1, then averaged Eq. 6.2), and then rescaled again Eq. 6.1, giving place to the previously defined efficiency. In this case, the obtained $p$-values using the Student's t-testin pairs shows that the interaction tools are significantly different except for two combinations (Table 6.2). In those cases, it is not possible to determine which one of them is statistically better or worse.

\begin{tabular}{l|lllll|}
\multicolumn{5}{c}{ CR } & \multicolumn{3}{c}{} \\
\cline { 2 - 4 } MS & $10^{-11}$ & \multicolumn{1}{l}{ MS } & & \\
LG & $10^{-5}$ & $10^{-15}$ & LG & & \\
RG & 0.18 & $10^{-12}$ & $10^{-5}$ & RG & \\
VC & $10^{-22}$ & $10^{-5}$ & $10^{-26}$ & $10^{-20}$ & VC \\
GV & $10^{-3}$ & $10^{-17}$ & 0.13 & 0.02 & $10^{-26}$ \\
\cline { 2 - 4 } & & & & &
\end{tabular}

Table 6.2. Pair-wise $p$-values to show the significance between the results of efficiency in the interaction tool test. Relations in red do not reject the null hypothesis. CR: controller, MS: master-slave, LG: local gestures, RG: remote gestures, VC: voice commands, GV: gestures and voice.
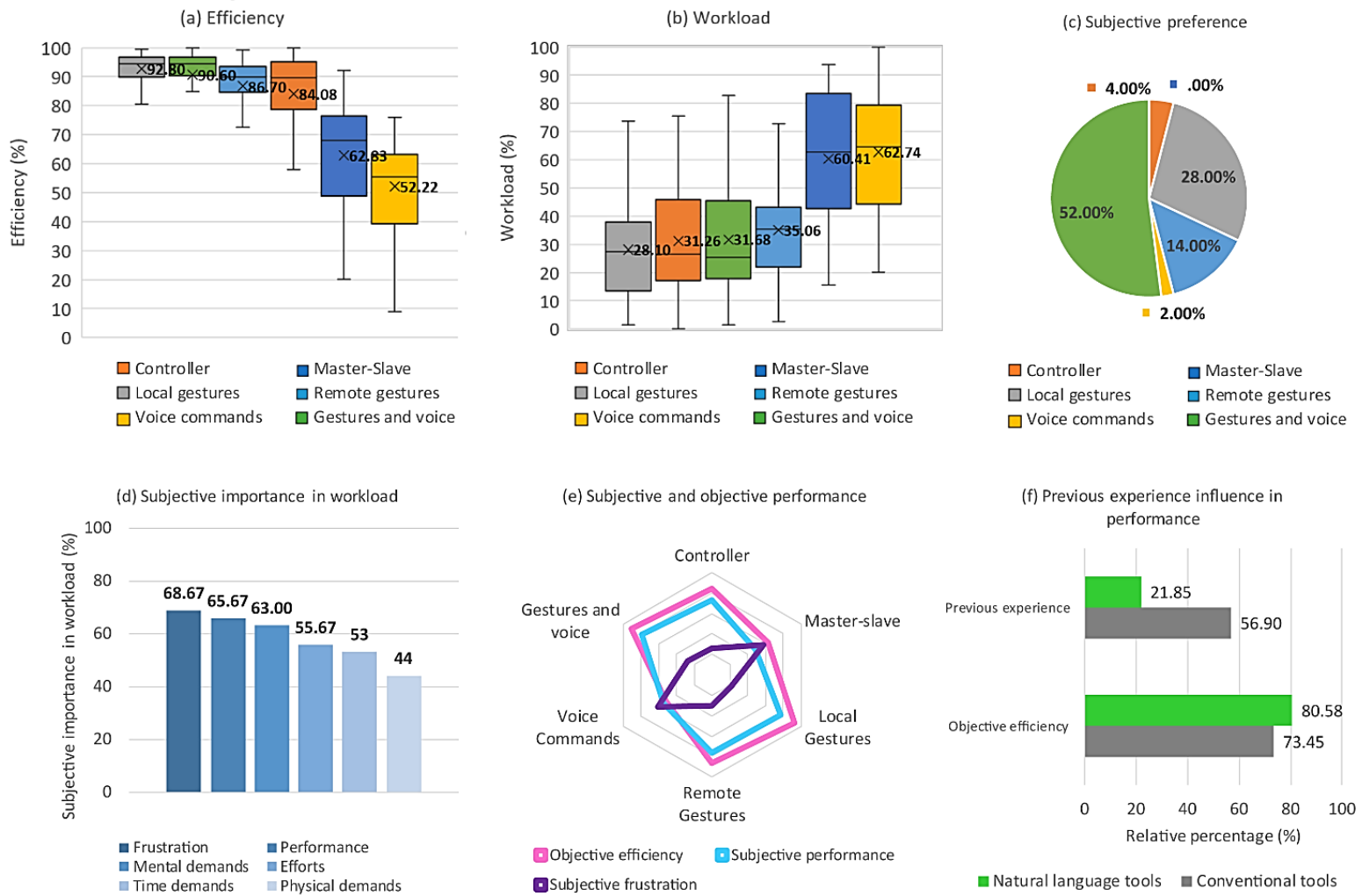

Figure 6.12. Results for the interaction-tools test. (a) Efficiency in terms of precision and speed, (b) workload, (c) subjective preference, (d) subjective importance of variables in workload, (e) subjective and objective performance comparison and ( $f$ ) previous experience influence in performance. 
Therefore, as seen in Figure 6.12a, the efficiency is indistinguishably the best for both local gestures and gestures and voice, with mean values of around $90 \%$. They are closely followed by remote gestures and the controller, also indistinguishable between each other with efficiencies of around $85 \%$ on average. And finally, the master-slave and the voice commands are respectively the ones with less objective efficiency.

On the other hand, the most common method to determine the workload (W) of a mission is the NASA Task Load Index (NASA-TLX) [275, 279]. It is based on rating 6 variables related to the influence of workload: mental demands, physical demands, temporal demands, performance, efforts and frustration. These parameters were first defined to ease their comprehension and then, the users could evaluate them using natural numbers $\left(w_{i}=[0,20]\right)$. On the other hand, the participants ordered from lowest to highest the subjective relevance of such parameters in workload $\left(r_{i}=[0,5]\right)$, so the obtained scores were weighted and normalized according to Eq. 6.4.

$$
W=\frac{\sum_{i=1}^{6} w_{i} r_{i}}{\sum_{i=1}^{6} 20 r_{i}}
$$

Eq. 6.4

Afterwards, the resulting data is again rescaled Eq. $6.1 \mathrm{in}$ order to facilitate comparisons between strategies. Again, the p-values using the Student's t-test in pairs have been analysed to objectively make reliable comparisons from the results showed in Figure 6.12b (Table 6.3).

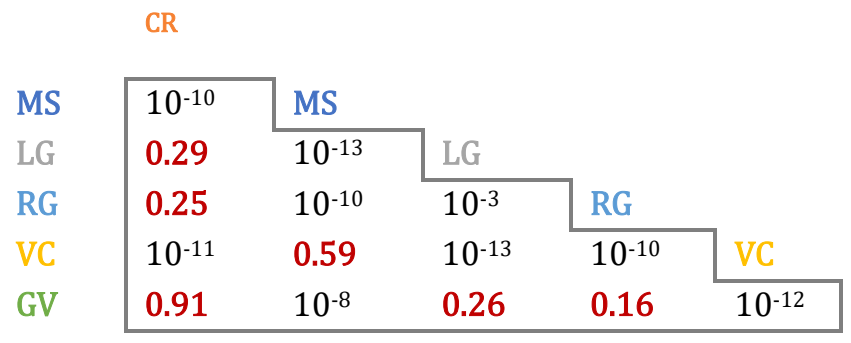

Table 6.3. Pair-wise p-values to show the significance between the results of the workload in the interaction tools test. Relations in red do not reject the null hypothesis. CR: controller, MS: master-slave, LG: local gestures, RG: remote gestures, VC: voice commands, GV: gestures and voice.

Thus, local gestures, controller, gestures and voice and remote gestures have half of the workload compared to master-slave and voice commands with mean values of approximately $32 \%$ and $61 \%$, respectively.

Besides, the participants selected the frustration as the most important factor to influence workload, followed by performance, mental demands, efforts, time demands, and physical demands as shown in Figure 6.12d. Furthermore, it is possible to compare the objective efficiency represented in Figure 6.12a with the subjective performance obtained from the workload test. Surprisingly, the participants were very accurate evaluating their performance and it was found to be highly influenced by the level of frustration (Figure $6.12 \mathrm{e})$.

As explained in Table 6.1, the participants were asked about their previous experience in VR and AR, robotics and videogames. If that experience is averaged and plotted against the objective mean efficiency for both tests, the variables are found as directly proportional (Figure 6.13). Therefore, previous experience can be understood as a very important factor to achieve a high level of efficiency. 


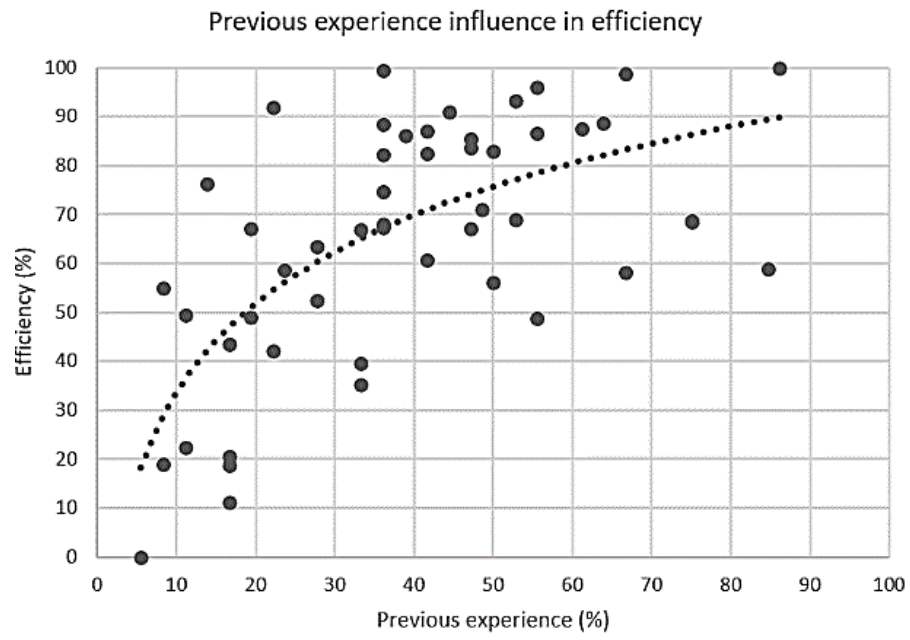

Figure 6.13. Previous experience influence in efficiency both for the human-robot interfaces and the interaction tools experiments.

Although the master-slave strategy is controlled by gestures, it has been inspired by the physical architecture. Therefore, this work proposes to consider this tool as conventional, together with the controller one. In this context, the results show that despite having less experience with natural language than conventional tools, the participants performed better using the first ones against the last ones (Figure $6.12 f)$.

Finally, and regardless of previous results, the participants were asked to choose among their preferred interaction tool to teleoperate hyper-redundant robots. More than half of the participants, exactly $52 \%$, chose the gestures and voice strategy, followed by local gestures with $28 \%$ and remote gestures with $14 \%$ (Figure 6.12c). If the number of conventional and natural-language tools were the same, $92.31 \%$ of the participants would have preferred to use a natural-language tool against a conventional one. 


\subsection{Applications}

The first industrial revolution used water and steam to mechanize production, the second used electric energy to create mass production and the third used electronics and information technology to automate production. Industry 4.0 is the next revolution, and it does not aim to produce more but to customize and optimize the production process. Robotics and immersive realities are considered as some of the most important technologies to achieve that goal [280].

This section will present an analysis of an inspection task in a real environment using such technologies. Specifically, it consists on the inspection of a boiler room looking for anomalies. These environments can be hazardous and unstructured with complex pipe structures, so using dexterous robots can be a good idea without the presence of human beings. However, these tasks usually involve high risks and sometimes require to take unexpected and complex decisions. Thus, human intervention is usually desirable from remote and safe locations. Hyper-redundant robots are devised for this kind of tasks. Additionally, recent work claims that immersive technologies can be the best solution to ease their spatial understanding and command their shape in the three-dimensional space [15].

As a result, the system setup is comprised of two workspaces: the robot is located in the boiler room while an operator safely commands its movements from a safe and remote location. In the boiler room (Figure 6.14a), the robot is disposed to perform the inspection task with 2 on-board sensors (Figure 6.14b): an RGB camera and a thermal camera located on its end-effector (Figure 6.14c). On the other hand, an operator is located in a remote location, provided with a head-mounted display (HMD) to command the robot movements using Mixed Reality. Specifically, the operator will be introduced in a virtual environment previously reconstructed in 3D using structured-light techniques (Figure 6.14d). This virtual scenario was recreated mimicking the real one in order to increase the situational awareness of the operator (Figure 6.14e). The HMD used is the HTC Vive headset in conjunction with the Leap Motion controller to track the hand movements (Figure 6.14F). As a result, the operator has visual, aural and vestibular feedback to effectively command the robot movements. Making an appropriate selection of the sensors in both spaces is crucial to obtain a good teleoperation performance.
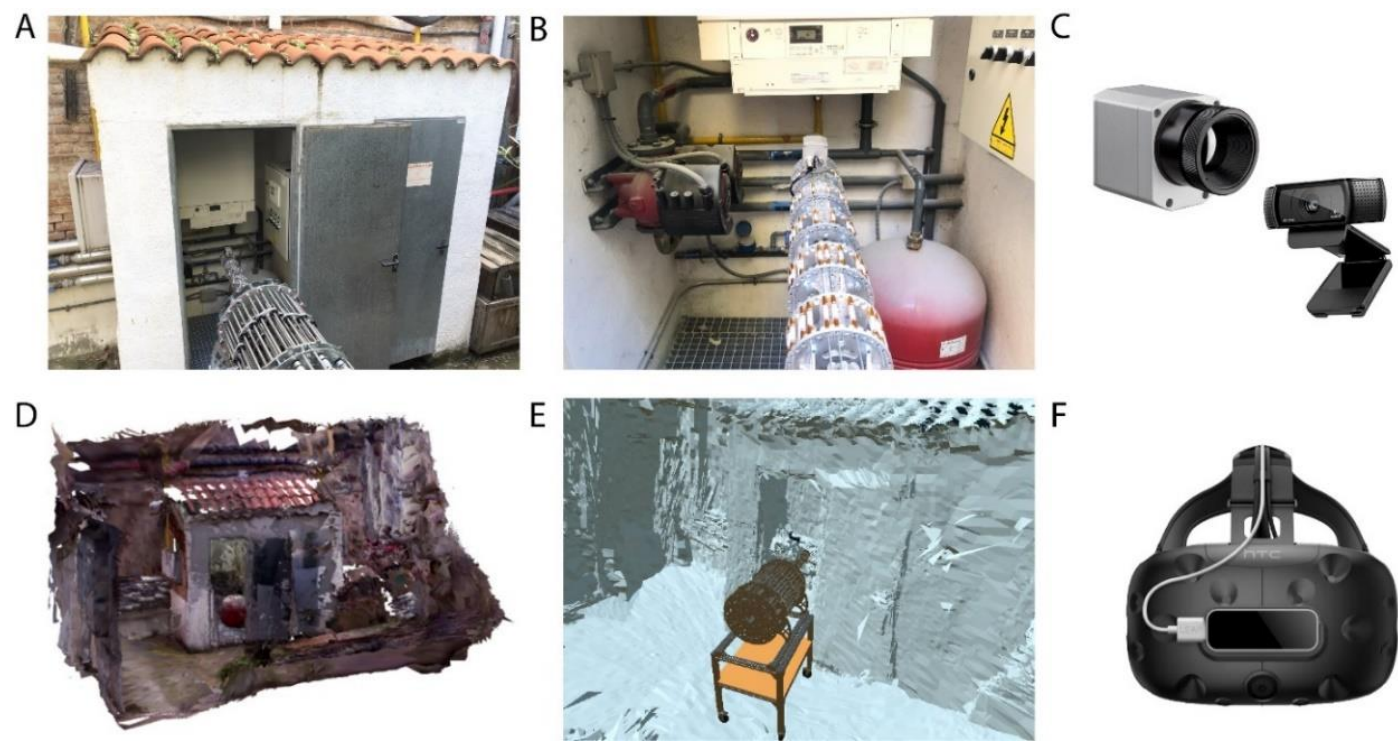

Figure 6.14. (a) Boiler room with the robot prepared to perform an inspection. (b) First-person view of the robot and the thermal camera located in the end-effector. (c) Two sensors were used in the real environment: an Optrix PI thermal camera and a Logitech RGB camera. (d) On the other hand, the real scenario was reconstructed using a Kinect and the software RTAB-Map. (e) Then, it was imported in Virtual Reality with a model of the robot. (f) The operator uses the HTC Vive VR headset and the Leap Motion controller to teleoperate the real robot from a safe location. 
Also, communications and delay management are very important since they determine if the system is suited for real-time applications. Thus, a bilateralarchitecture was implemented using sockets and a TCP/IP connection. Furthermore, the designed teleoperation follows a position-position scheme. In other words, the robot will move to a determined position previously commanded by the user. Although this scheme is very simple, it is the best suited for this application, since the size relation of the master-slave is similar, there are small limitations in the slave speed, a high level of telepresence is required, and the task involves a high number of DoF [265].

In summary, the operator will use Mixed Reality to move an exact replica of the robot in the virtual environment. The resulting telepresence is very high given that the robot size, movements, restrictions and its environment in the virtual world match with the ones in the real world (Figure 6.15a). The user will be able to use the bare hands to interact with the virtual robot so the real one adopts the same pose using shape-control techniques. Then, once the desired configuration is ready, the operator pushes a virtual button and the signal is sent to the real one. As a result, the real robot moves according to the configuration commanded in the virtual world. With this strategy, the user is safe from a remote distance while, at the same time, has the perception of being in the boiler room. Simultaneously, a video streaming with the output from the thermal camera allows to supervise any abnormal temperatures inside the boiler room (Figure $6.15 b)$. Thanks to the high versatility of the hyper-redundant robot, it can orient the end-effector with high dexterity whereas a traditional, non-redundant one would have struggled to perform the same task.
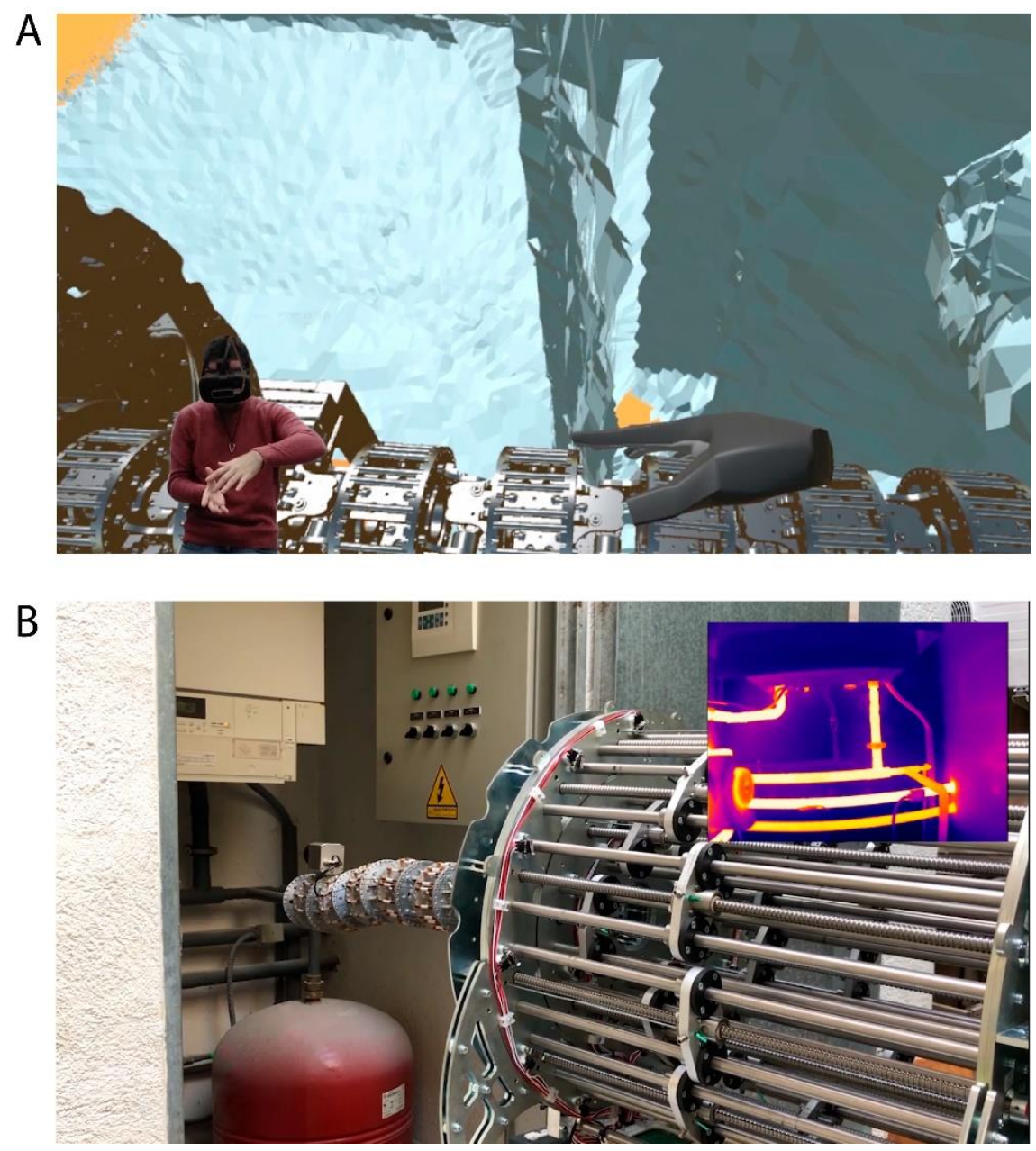

Figure 6.15. (a) The operator performs a shape control using his bare hands to interact with the virtual robot, a replica of the real one with the same kinematics and physical restrictions. (b) Using Mixed Reality, the real robot (slave) is moved adopting the same pose as the virtual one (master). The thermal camera located in the end-effector let the operator supervise the temperatures of the boiler room from a safe and remote location. 
As a result of the inspection and using the RGB camera, it is concluded that there is no appreciable damage, but just some debris that may cause malfunction problems in the pumps in the long-term period. On the other hand, the hyper-redundant robot allowed to inspect the constrained space using the thermal camera. The pipes and pumps temperature was adequate and homogeneous, which implies that the boiler is performing appropriately. However, the heat seemed to undesirably expand by conduction through small metal elements and the room walls deteriorating the infrastructure. Thus, thermal insulation was recommended to be applied in certain locations to prevent future damages.

In addition to previous developments based in Virtual Environments to command the robots, a different headset was used to create Mixed Reality experiences based in Augmented Reality, the Hololens headmounted display.

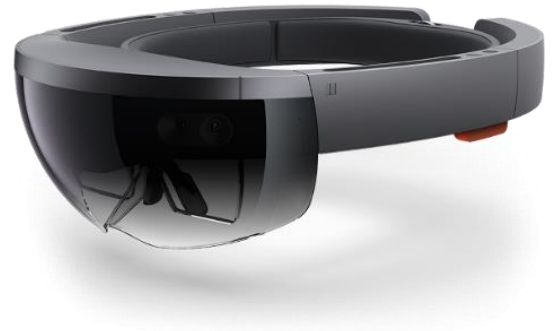

Figure 6.16. The Hololens device, a head mounted display intended for Augmented and Mixed Reality experiences.

As a remainder, in Augmented Reality (AR) the virtual objects are overlapped as holograms in the real world. Then, commanding the movements of hyper-redundant, continuous and soft robots with this technology requires the appearance of the user in the same environment as the robot. Therefore, this kind of practices are not intended to control robots located in hazardous environments but to enrich their teleoperation for in situ supervision. The use of AR can be extremely useful to perceive the complex shapes that this robots can adopt in order to convey the best strategy depending on each application. The Mixed Reality Toolkit library and the software Unity $3 D$ were used to develop this scenario. The soft robot Kyma was also controlled in real-time using this strategy and the results were very satisfactory.

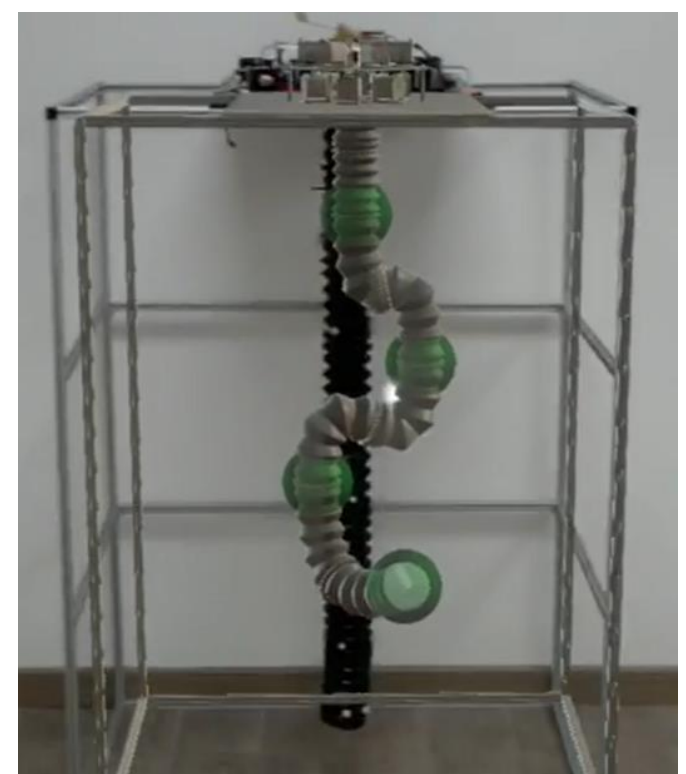

Figure 6.17. Hologram overlapped to the real robot in order to command a shape-control using the Hololens device. Both gestures and voice commands were used to move the hologram. 


\subsection{Discussion}

This section aims to clear the path to focus the future research of hyper-redundant robot's teleoperation. This kind of robots have a lot of degrees of freedom, so their spatial understanding is often difficult to be perceived. And teleoperation is a very important field in robotics since it is essential for several applications such as surgery, spatial missions, inspection or surveillance. A bilateral communication based on a positionposition scheme has selected as the most adequate to teleoperate hyper-redundant robot applications.

This section aimed to solve whether immersive 3D interfaces are better or worse than conventional 2D ones in order to teleoperate hyper-redundant robots. Experiments with 50 participants show that immersive interfaces exhibit 33.55\% mean higher efficiency in terms of speed and precision and $73.71 \%$ mean higher situational awareness than conventional ones. As a result, immersive interfaces were chosen to teleoperate this kind of robots by $94 \%$ of the participants.

On the other hand, some interaction tools were tested by the same population in order to determine which strategy is better suited for the same purpose within an immersive reality. A total of 6 methods were developed: controller, master-slave, local gestures, remote gestures, voice commands and gestures and voice. In this case, efficiency is indistinguishable the best for both local gestures and gestures and voice, with mean values of around $90 \%$, closely followed by remote gestures and the controller. Also, a workload test showed that local gestures, controller, gestures and voice and remote gestures have half of the workload than the other two approaches.

Moreover, the participants were very accurate in evaluating their performance, which was found to be highly influenced by the level of frustration. Moreover, results showed that natural-language tools are more intuitive since exhibited higher performance despite the less previous experience. Finally, more than half of the users chose the gestures and voice strategy as the preferred one. This is understandable since human beings are used to communicate by simultaneously gesticulating and speaking. This is closely related to the Principle of Least Astonishment (POLA), which states that a user interface should behave in the way that most users will expect it to behave. Thus, it can be deduced that this combined strategy was the most intuitive while, at the same time, the distribution of tasks to different stimulus did not increase the subjective workload to the users. Moreover, $92.31 \%$ selected an interaction tool based on natural language against a conventional one. Future work in this matter should be focused on proposing and classifying new interaction strategies in order to select the most appropriate, depending on the hyper-redundant robot specifications and its applications. Conducted experiments with a real hyper-redundant robot for inspection tasks in a boiler room show the efficiency and accuracy of immersive technologies and natural language to perform a remote teleoperation. Therefore, it can be concluded that these tools should play an important role in successive approaches towards hyper-redundant robots and their teleoperation.

Additionally, the teleoperation was tested in a real environment within the framework of Industry 4.0. Specifically, it was teleoperated to conduct an inspection task in a boiler room using Mixed Reality in realtime. These practices are very useful when the real environment is potentially hazardous or adverse. Both the boiler room and the robot were replicated in a virtual world, where the user could use the bare hands to interact with the virtual robot and command the movements of the real one. Many sensors were used for this application from encoders, thermal and RGB cameras, a head-mounted display with 6 DoF or a handtracking visual system. Making an appropriate selection of these sensors is crucial to obtain an optimal teleoperation performance. Consequently, the operators experienced high situational awareness and performed with high efficiency from a remote and safe location. Results from the inspection task revealed that the boiler was functioning adequately whereas application of thermal insulation was recommended in certain locations to prevent future damages. 


\section{Repercussion}

This chapter precedes the conclusions to previously analyse the impact of this thesis. First, some of the most potential applications and benefits derived from this work are explained. Then, a brief analysis will explain the sustainability and ethic queries that can be inferred from the research activities, methods and future implications. Next, some of the most relevant achievements obtained during the execution of this thesis are mentioned. And finally, electronic supplementary materials will be introduced to gather some explanatory videos that emphasize the contributions of this work. 


\subsection{Applications and benefits}

Future applications and benefits derived from the design, modelling, control and teleoperation of hyperredundant, continuous and soft robots are a reality now. Previous works in the state-of-the-art have already supported some of the following.

- In medicine, for surgical interventions such as laparoscopies, endoscopies, NOTES (Natural Orifice Transluminal Endoscopy Surgery), neurological and cardiovascular procedures. Also for the diagnosis of illnesses at early stages or for rehabilitation purposes, since this robots can safely comply with their environment. Additionally, they can be used for veterinary surgical interventions and diagnosis in some animals. Interestingly, their snake-like morphology can also be used to subdue rabid animals with instinct reactions towards these reptiles.

- They can also be extremely useful for inspection of industrial facilities such as silos, tubes or turbines. Also, for operative tasks such as welding or any required task in hazardous environments like boiler rooms or nuclear core reactors.

- In the mine sector, for the detection of toxic elements, setting up explosives or exploring through small cracks. Moreover, for the detection of bombs, toxic elements or drug stash in difficult-toaccess areas. And in the agriculture, these robots could help to safely comply to grab delicate fruits or vegetables.

- Cartographies and 3D reconstruction of volumes in constrained spaces such as for archaeology purposes: exploration of delicate chambers, vessels, sarcophagi, boxes or skulls, among many others.

- Mobile highly articulated robots can be useful for search and rescue tasks such as accessing to contaminated areas after a natural or human disaster, servicing for underground tanks, inspecting underwater facilities or for military applications such as the access into demolished areas.

- The addition of growing capabilities in some of the cases let them outperform for such as for the deployment of antennas in the telecommunications sector, detection and extinction of fires, exploration of coral reefs, locomotion purposes or even to grab space debris.

- This kind of robots can be a great accessory manipulator to support activities where the fault tolerance is highly demanded. For example, they can be useful for space exploration since they can reconfigure if some of the actuators fail. Additionally, the study of highly-articulated mechanisms is not only beneficious just for the robotics sector. The ongoing research can be used for multiple other ideas such as for creating space launchers or studying compliant materials and properties.

- For research and educational purposes, highly articulated robots are quite interesting since they exploit the traditional kinematics of traditional and industrial manipulators to their maximum expression.

Therefore, it can be concluded that the area of impact of the presented technologies is very wide. Besides, it is a specialization that can provide significant contributions to numerous other scientific areas. 


\subsection{Sustainability and ethics}

The concept of sustainable development is the organizing principle for meeting human development goals while simultaneously sustaining the ability of natural systems to provide the natural resources and ecosystem services based upon which the economy and society depend (Figure 7.1).

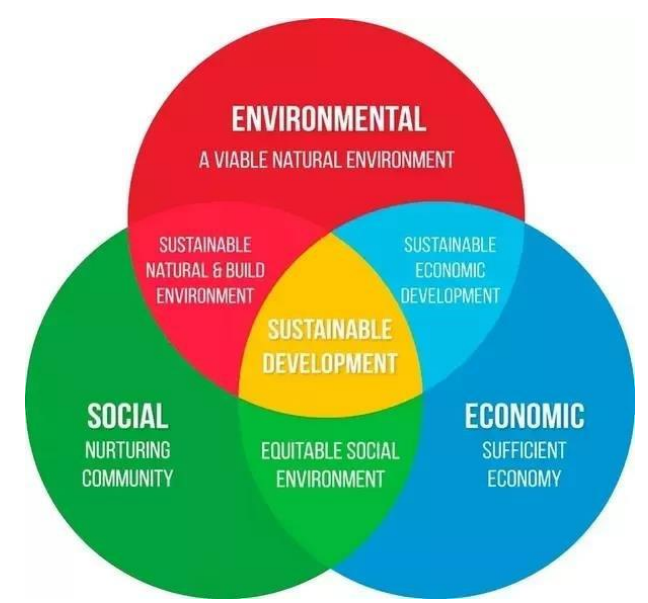

Figure 7.1. Main principles of the sustainable development.

It is encouraged to design hyper-redundant, continuous and soft robots as biomimetic as possible, especially in those situations where they need to perform in natural environments or interacting with living systems. In this context, soft and continuous robots would usually be better suited than rigid ones to allow safe and compliant interactions with delicate objects.

Additionally, it is important to emphasize the importance of using raw materials and products obtained under a Fair Trade framework for the construction of these robots. Furthermore, they need to be economically affordable and their use should be equally spread around the world, avoiding any kind of discrimination.

From the social perspective, the use of hyper-redundant, continuous or soft robots should only be used to directly improve the quality of life of the people (Figure 7.2). The contributions of this work are not authorised to be used by any means for warlike purposes or any other project that could harm, either directly or indirectly, the welfare of mankind.

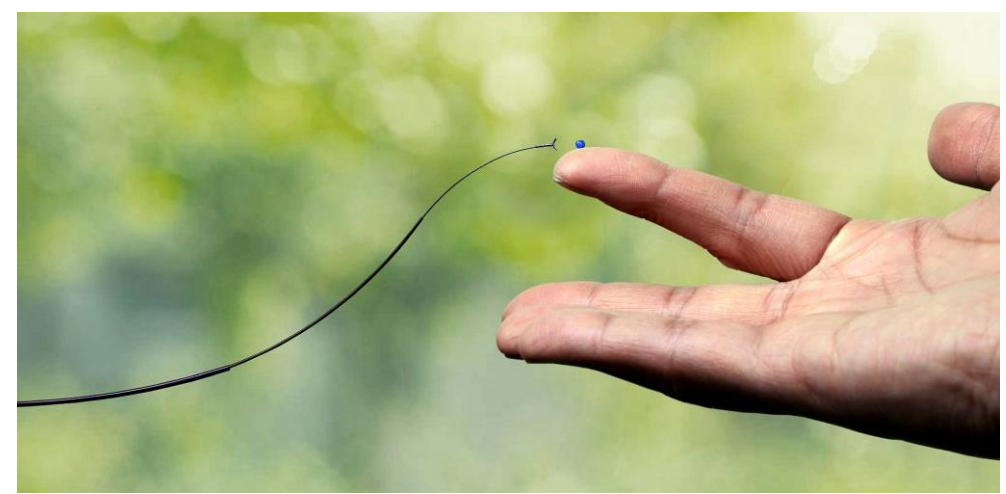

Figure 7.2. Concentric tube continuum robot. Source: Continuum Robots Laboratory, University of Toronto Mississauga.

The conjunction of these basic principles are the only warranty to an equitable, liveable and viable future surrounded by technology. 


\subsection{Achievements}

This section summarizes some of the most relevant achievements and curricular experience obtained during the execution of this thesis from relevant previous works, publications, conferences, projects, scholarships, teaching courses, participation as reviewer or supervision of BSc and MSc thesis.

\subsubsection{Relevant previous works}

This PhD thesis is the continuation of the BSc and MSc thesis presented by the same author in previous years. Therefore, the scope of this thesis has been studied by the author for 6 years to the presentation of this document (Table 7.1).

\begin{tabular}{llllll}
\hline Title & Author & Supervisor & University \\
\hline 1. & $\begin{array}{l}\text { Robot Laparoscópico Biomimético e Híper- } \\
\text { Redundante: Análisis Preliminar de Materiales }\end{array}$ & A Martín-Barrio & J.C Marinero & Universidad de Valladolid \\
& $\begin{array}{l}\text { Magnéticos, Guiado mediante Visión Artificial y } \\
\text { Primer Estudio de su Diseño. }\end{array}$ & & \\
2 & $\begin{array}{l}\text { Control Cinemático de Robots Híper- } \\
\text { Redundantes. El Algoritmo Natural-CCD }\end{array}$ & A Martín-Barrio & A Barrientos & Universidad Politécnica de Madrid \\
\hline
\end{tabular}

Table 7.1. Previous BSc and MSc thesis were continued for the execution of this PhD thesis.

\subsubsection{Publications}

This thesis is result of the publication of the following articles in scientific journals. These articles have direct relation with this work (Table 7.2).

\begin{tabular}{|c|c|c|c|c|c|c|c|}
\hline & Title & Authors & Journal & Year & $\begin{array}{l}\text { Impact } \\
\text { factor }\end{array}$ & Quartile & Link \\
\hline 1. & $\begin{array}{l}\text { The Natural-CCD Algorithm, a Novel } \\
\text { Method to Solve the Inverse } \\
\text { Kinematics of Hyper-redundant and } \\
\text { Soft Robots }\end{array}$ & $\begin{array}{l}\text { A Martín-Barrio } \\
\text { A Barrientos } \\
\text { J del Cerro }\end{array}$ & Soft Robotics & 2018 & 6.403 & $\begin{array}{l}\text { Q1 } \\
\text { (Robotics) }\end{array}$ & $\begin{array}{l}\text { https://doi.or } \\
\text { g/10.1089/sor } \\
0.2017 .0009\end{array}$ \\
\hline 2. & $\begin{array}{l}\text { Emerging Behaviours from Cyclical, } \\
\text { Incremental and Uniform } \\
\text { Movements of Hyper-Redundant and } \\
\text { Growing Robots }\end{array}$ & $\begin{array}{l}\text { A Martín-Barrio } \\
\text { J del Cerro } \\
\text { A Barrientos } \\
\text { H Hauser }\end{array}$ & $\begin{array}{l}\text { Mechanics and } \\
\text { Machine Theory }\end{array}$ & 2020 & 3.535 & $\begin{array}{l}\text { Q1 } \\
\text { (Mechanical } \\
\text { Engineering) }\end{array}$ & Under review \\
\hline 3. & $\begin{array}{l}\text { Design of a Hyper-Redundant Robot } \\
\text { and Teleoperation using Mixed } \\
\text { Reality }\end{array}$ & $\begin{array}{l}\text { A Martín-Barrio } \\
\text { J.J. Roldán } \\
\text { I. Rodríguez } \\
\text { J. del Cerro } \\
\text { A Barrientos }\end{array}$ & Sensors & 2020 & 3.031 & $\begin{array}{l}\text { Q1 } \\
\text { (Instruments and } \\
\text { Instrumentation) }\end{array}$ & $\begin{array}{l}\text { https://doi.or } \\
\text { g/10.3390/s20 } \\
082181\end{array}$ \\
\hline 4. & $\begin{array}{l}\text { Application of Immersive } \\
\text { Technologies and Natural Language } \\
\text { to Hyper-Redundant Robot } \\
\text { teleoperation }\end{array}$ & $\begin{array}{l}\text { A Martín-Barrio } \\
\text { JJ Roldán } \\
\text { S Terrile } \\
\text { J del Cerro } \\
\text { A Barrientos }\end{array}$ & Virtual Reality & 2019 & 2.906 & $\begin{array}{l}\text { Q1 } \\
\text { (Computer } \\
\text { Science, Software } \\
\text { Engineering) }\end{array}$ & $\begin{array}{l}\text { https://doi.or } \\
\text { g/10.1007/s10 } \\
\text { 055-019- } \\
00414-9\end{array}$ \\
\hline 5. & $\begin{array}{l}\text { Modelling the soft robot Kyma using } \\
\text { finite-element analysis }\end{array}$ & $\begin{array}{l}\text { A Martin-Barrio } \\
\text { S Terrile } \\
\text { M Díaz-Carrasco } \\
\text { J del Cerro } \\
\text { A Barrientos }\end{array}$ & $\begin{array}{l}\text { Computer Graphics } \\
\text { Forum }\end{array}$ & 2020 & 2.373 & $\begin{array}{l}\text { Q2 } \\
\text { (Computer } \\
\text { Science, Software } \\
\text { Engineering) }\end{array}$ & $\begin{array}{l}\text { https://doi.or } \\
\text { g/10.1111/cgf. } \\
14026\end{array}$ \\
\hline 6. & $\begin{array}{l}\text { Hyper-Redundant Robots: } \\
\text { Classification, State-of-the-Art and } \\
\text { Issues }\end{array}$ & $\begin{array}{l}\text { A Martin-Barrio } \\
\text { S Terrile } \\
\text { A Barrientos } \\
\text { J del Cerro }\end{array}$ & $\begin{array}{l}\text { Revista } \\
\text { Iberoamericana de } \\
\text { Automática e } \\
\text { Informática } \\
\text { industrial }\end{array}$ & 2018 & 1.313 & $\begin{array}{l}\text { Q3 } \\
\text { (Automation and } \\
\text { Control systems) }\end{array}$ & $\begin{array}{l}\text { https://doi.or } \\
\text { g/10.4995/riai. } \\
2018.9207\end{array}$ \\
\hline
\end{tabular}




\subsubsection{Other publications}

Other articles with limited relation to this thesis were published during its execution (Table 7.3).

\begin{tabular}{|c|c|c|c|c|c|c|c|}
\hline & Title & Authors & Journal & Year & $\begin{array}{l}\text { Impact } \\
\text { factor }\end{array}$ & Quartile & Link \\
\hline 1. & $\begin{array}{l}\text { A training system for Industry } 4.0 \\
\text { operators in complex assemblies } \\
\text { based on virtual reality and process } \\
\text { mining }\end{array}$ & $\begin{array}{l}\text { JJ Roldán } \\
\text { E Crespo } \\
\text { A Martín-Barrio } \\
\text { E Peña-Tapia } \\
\text { A Barrientos }\end{array}$ & $\begin{array}{l}\text { Robotics and } \\
\text { Computed- } \\
\text { Integrated } \\
\text { Manufacturing }\end{array}$ & 2019 & 4.071 & Q1 (Robotics) & $\begin{array}{l}\text { https://doi.org/1 } \\
0.1016 / \text { j.rcim.20 } \\
19.05 .004\end{array}$ \\
\hline 2. & $\begin{array}{l}\text { Multi-robot interfaces and operator } \\
\text { situational awareness: Study of the } \\
\text { impact of immersion and prediction }\end{array}$ & $\begin{array}{l}\text { JJ Roldán } \\
\text { E Peña-Tapia } \\
\text { A Martín-Barrio } \\
\text { M Olivares-Méndez } \\
\text { J del Cerro } \\
\text { A Barrientos }\end{array}$ & Sensors & 2017 & 2.475 & $\begin{array}{l}\text { Q2 (Instruments } \\
\text { and } \\
\text { instrumentation) }\end{array}$ & $\begin{array}{l}\text { https://doi.org/1 } \\
0.3390 / \text { s } 170817 \\
20\end{array}$ \\
\hline
\end{tabular}

Table 7.3. Other relevant publications with limited relation to this thesis.

\subsubsection{Conferences}

This thesis also lead to the assistance as a speaker in the following conferences, which are closely based on this work (Table 7.4).

\begin{tabular}{|c|c|c|c|c|c|}
\hline & Title & Authors & Conference & Location & Year \\
\hline 1. & $\begin{array}{llr}\text { Proyecto } & \text { PRIC: } \quad \text { Protección } \\
\text { Robotizada } & \text { de } & \text { Infraestructuras } \\
\text { Críticas } & & \end{array}$ & $\begin{array}{l}\text { JJ Roldán } \\
\text { M Garzón } \\
\text { J de León } \\
\text { D Garzón } \\
\text { A Martín-Barrio } \\
\text { S Terrile } \\
\text { P García-Auñón } \\
\text { J del Cerro } \\
\text { C Rossi } \\
\text { A Barrientos }\end{array}$ & Jornadas Nacionales de Robótica & Valencia (Spain) & 2017 \\
\hline 2. & $\begin{array}{l}\text { New trends in hyper-redundant, } \\
\text { continuous and soft robots }\end{array}$ & A Martín-Barrio & SoftLab - Bristol Robotics Laboratory & Bristol (UK) & 2019 \\
\hline 3. & $\begin{array}{l}\text { Immersive technologies to hyper- } \\
\text { redundant robot teleoperation }\end{array}$ & A Martín-Barrio & $\begin{array}{l}\text { Robotics for remote manipulation } \\
\text { activities in space \& on earth. European } \\
\text { Space Agency. }\end{array}$ & Oxford (UK) & 2019 \\
\hline
\end{tabular}

Table 7.4. Conference presented in the context of this PhD thesis.

\subsubsection{Other conferences}

Additionally, other conferences with limited relation to this thesis were published during its execution (Table 7.5).

\begin{tabular}{|c|c|c|c|c|c|}
\hline & Title & Authors & Conference & Location & Year \\
\hline 1. & $\begin{array}{l}\text { Interfaz de control para un robot manipulador } \\
\text { mediante realidad virtual }\end{array}$ & $\begin{array}{l}\text { E Peña-Tapia } \\
\text { JJ Roldán } \\
\text { M Garzón } \\
\text { A Martín-Barrio } \\
\text { A Barrientos }\end{array}$ & XXXVIII Jornadas de Automática & Gijón (Spain) & 2017 \\
\hline 2. & $\begin{array}{l}\text { Realidad virtual y minería de procesos } \\
\text { aplicadas a la formación de operarios en tareas } \\
\text { de ensamblado complejo }\end{array}$ & $\begin{array}{l}\text { E Crespo } \\
\text { JJ Roldán } \\
\text { A Martín-Barrio } \\
\text { J del Cerro } \\
\text { A Barrientos }\end{array}$ & $\begin{array}{l}\text { IV Seminario de Innovación } \\
\text { Docente en Automática }\end{array}$ & León (Spain) & 2018 \\
\hline
\end{tabular}

Table 7.5. Other relevant conferences with limited relation to this thesis. 


\subsubsection{Projects}

Spanish and European projects defined the framework and the funding resources to finish this work (Table 7.6).

\begin{tabular}{|c|c|c|c|}
\hline & Title & Code & Funder \\
\hline 1. & $\begin{array}{l}\text { Robotic Protection for Critical Infrastructures } \\
(P R I C)\end{array}$ & DPI2014-56985-R & $\begin{array}{l}\text { Ministry of Economy and } \\
\text { Competitiveness of the Government of Spain }\end{array}$ \\
\hline 2. & $\begin{array}{l}\text { Robocity2030-DIH-CM, Madrid Robotics Digital } \\
\text { Innovation Hub }\end{array}$ & S2018/NMT-4331 & $\begin{array}{l}\text { Programas de Actividades I+D en la Comunidad de } \\
\text { Madrid and Structural Funds of the EU }\end{array}$ \\
\hline 3. & UPM Program Project & VJIDOCUPM18JCG & Universidad Politécnica de Madrid \\
\hline
\end{tabular}

Table 7.6. Main projects that funded this thesis.

\subsubsection{Scholarships}

The presented work can be categorized as an International PhD Thesis since some contributions were developed in the United Kingdom. The author of this project was granted with a scholarship to research about hyper-redundant and growing robots in the University of Bristol for 3 months (Table 7.7). As a result, a publication was published.

\begin{tabular}{llllll}
\hline & Title & Beneficiary & Supervisors & University \\
\hline 1 & $\begin{array}{l}\text { Ayuda del Consejo Social para el Fomento de la } \\
\text { Formación y la Internacionalización de } \\
\text { Doctorandos (2019-19) }\end{array}$ & A Martín-Barrio & H Hauser & Aniversity of Bristol \\
\end{tabular}

Table 7.7. Scholarship granted to obtain the International Mention for this PhD thesis.

\subsubsection{Teaching courses}

The following courses were given in the integral context of the author as an educator (Table 7.8).

\begin{tabular}{llll}
\hline & Title & Location & Year \\
\hline 1. & Visión por computador & Centro de Automática y Robótica (CSIC-UPM) & 2017 \\
2. & Dinámica de sistemas & Universidad Politécnica de Madrid & 2018 \\
3. & Ingeniería en tus manos - Realidad Virtual & Universidad Politécnica de Madrid & 2019 \\
4. & Visión por computador & FIDAMC (Madrid) & 2019 \\
\hline
\end{tabular}

Table 7.8. Participation as assistant professor in educational courses.

\subsubsection{Reviewer}

As a contribution to the scientific field, the author of this thesis is reviewer of the following journals and conferences (Table 7.9).

\begin{tabular}{|c|c|c|}
\hline & Journal & Year \\
\hline 1. & Soft Robotics & 2017 \\
\hline 2. & Revista Iberoamericana de Automática e Informática Industrial & 2018 \\
\hline 3. & Jornadas Nacionales de Robótica & 2018 \\
\hline 4. & International Conference on Intelligent Robots and Systems & 2018 \\
\hline 5. & IEEE Transactions on Neural Networks and Learning Systems & 2018 \\
\hline 6. & IEEE Robotics and Automation Letters for RA-L IROS & 2019 \\
\hline 7. & International Journal of Robotics Research & 2019 \\
\hline
\end{tabular}

Table 7.9. Participation as reviewer in scientific journals and conferences. 


\subsubsection{Supervision of BSc and MSc projects}

Additionally, a total of $14 \mathrm{BSc}$ and MSc thesis were supervised with the professor Antonio Barrientos Cruz (Table 7.10).

\begin{tabular}{|c|c|c|c|c|}
\hline & Title & Author & Type & Year \\
\hline 1. & Desarrollo de un Robbot Manipulador Blando e Híper-Redundante & Cecilia Martínez Martín & TFG & 2017 \\
\hline 2. & $\begin{array}{l}\text { Modelado mediante Análisis de Elementos Finitos de Kyma, un Robot } \\
\text { Manipulador Blando }\end{array}$ & Montserrat Díaz-Carrasco Díaz & TFG & 2018 \\
\hline 3. & $\begin{array}{l}\text { Rediseño, Sensorización y Teleoperación mediante Realidad Aumentada de } \\
\text { Kyma, un Robot Manipulador Blando }\end{array}$ & Jesús Pareja Muñoz & TFG & 2018 \\
\hline 4. & $\begin{array}{l}\text { Generación y Evaluación de Secuencias Melódicas mediante Inteligencia } \\
\text { Artificial }\end{array}$ & Silvia Jiménez Gómez & TFG & 2018 \\
\hline 5. & $\begin{array}{l}\text { Planificación de un Robot Manipulador Móvil Autónomo para la Búsqueda y } \\
\text { Manipulación de una Válvula }\end{array}$ & David Gutiérrez Vivas & TFM & 2018 \\
\hline 6. & $\begin{array}{l}\text { Detección de Pallets mediante Técnicas de Visión por Computador y } \\
\text { Planificación de un Vehículo de Guiado Automático mediante Realidad Virtual }\end{array}$ & David Ramos Porres & TFM & 2018 \\
\hline 7. & Development of a Computer Vision System for use in Submarine Robots & Manuel Faysse & TFG & 2018 \\
\hline 8. & $\begin{array}{l}\text { Planificación de Robots Híper-Redundantes y Evaluación en Entornos } \\
\text { Complejos mediante Realidad Virtual }\end{array}$ & Álvaro Vara Mateos & TFG & 2019 \\
\hline 9. & Aplicación de Realidad Mixta para la Asistencia en Tareas de Ensamblaje & Javier Martín García & TFG & 2019 \\
\hline 10. & $\begin{array}{l}\text { Clasificación de Electrocardiogramas para la Detección Prematura de Infartos } \\
\text { mediante Redes Neuronales }\end{array}$ & Roberto Abia Carrasco & TFG & 2019 \\
\hline 11. & $\begin{array}{l}\text { Traductor Automático de la Lengua de Signos Española a Español mediante } \\
\text { Visión por Computadory Redes Neuronales }\end{array}$ & Laura Bachiller Matallana & TFG & 2019 \\
\hline 12. & $\begin{array}{l}\text { Teleoperación de un Robot Centauro mediante Realidad Mixta para tareas de } \\
\text { Búsqueda y Rescate }\end{array}$ & Adrián Barra Durán & TFG & 2020 \\
\hline 13. & $\begin{array}{l}\text { Teleoperación de Robots Híper-Redundantes mediante Tecnologías } \\
\text { Inmersivas y Lenguaje Natural }\end{array}$ & Agustín Ramírez Blanco & TFG & 2020 \\
\hline 14. & $\begin{array}{l}\text { Reducción de Orden de Modelos de Robots Blandos mediante el Método de } \\
\text { Elementos Finitos en Tiempo Real }\end{array}$ & Antonio del Abril de Mur & TFM & 2020 \\
\hline
\end{tabular}
Table 7.10. Co-supervised BSc and MSc projects during the execution of this thesis. 


\subsection{Electronic supplementary material}

Main sections and publications of this thesis are accompanied by an explanatory video as shown in Table 7.11.

\begin{tabular}{llc}
\hline & Title & Link \\
\hline S. 1 & Design of a Hyper Redundant Robot and Teleoperation using Mixed Reality & https://youtu.be/N8IqezQ9maA \\
S. 2 & Modelling the Soft Robot Kyma based on Real-Time Finite Element Method & https://youtu.be/i84Uu51tz6c \\
S. $\mathbf{3}$ & The Natural-CCD Algorithm & https://youtu.be/ulc0zI_od8o \\
S. $\mathbf{4}$ & Emerging Behaviours from Distributed Control of Hyper Redundant and Growing Robots & https://youtu.be/ORKp3toEmm8 \\
S. $\mathbf{5}$ & Immersive technologies and natural language for hyper-redundant robot teleoperation & https://youtu.be/aLtXwgCraSw \\
\hline
\end{tabular}

Table 7.11. Electronic supplementary material 

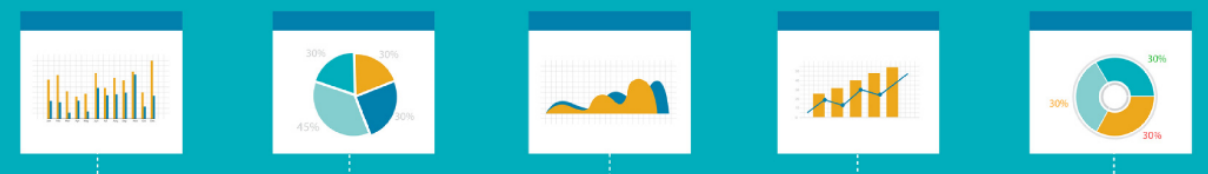

\section{Conclusions}

This section will summarize the main contributions, conclusions and future research lines of the present work. 


\subsection{Contributions}

Some of the most relevant contributions of this thesis are briefly enumerated as follows.

\begin{tabular}{l}
\hline Principles \\
\hline Proposal of a new definition of hyper-redundant robots. \\
Updated classification of hyper-redundant, continuous and soft robots. \\
Analysis of the current challenges for highly articulated systems. \\
Updated state-of-the-art in the fields of design, modelling, teleoperation and kinematic control.
\end{tabular}

Table 8.1. Contributions derived from basic principles

\begin{tabular}{l}
\hline Design \\
\hline Concept and design of Magno, a magnetically-actuated continuous robot with bending and prismatic capabilities.. \\
Participation in the conceptualization of Mach, a sturdy, robust and discrete hyper-redundant robot. \\
Design and construction of Kyma, a big, tendon-driven soft robot with bending and prismatic capabilities. \\
\hline
\end{tabular}

Table 8.2. Contributions derived from the design and construction of highly-articulated mechanisms.

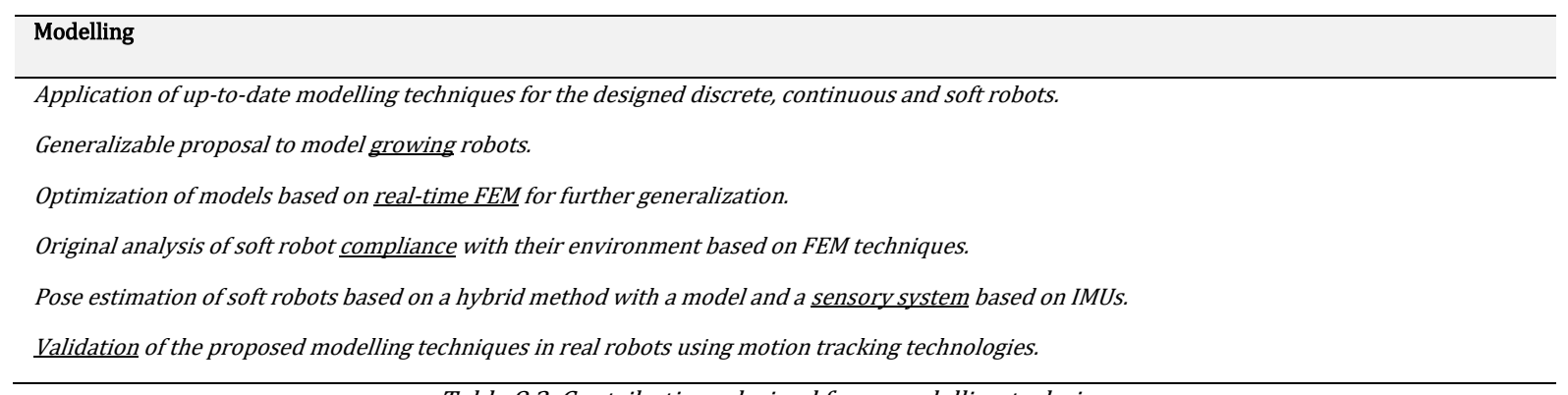

Table 8.3. Contributions derived from modelling techniques.

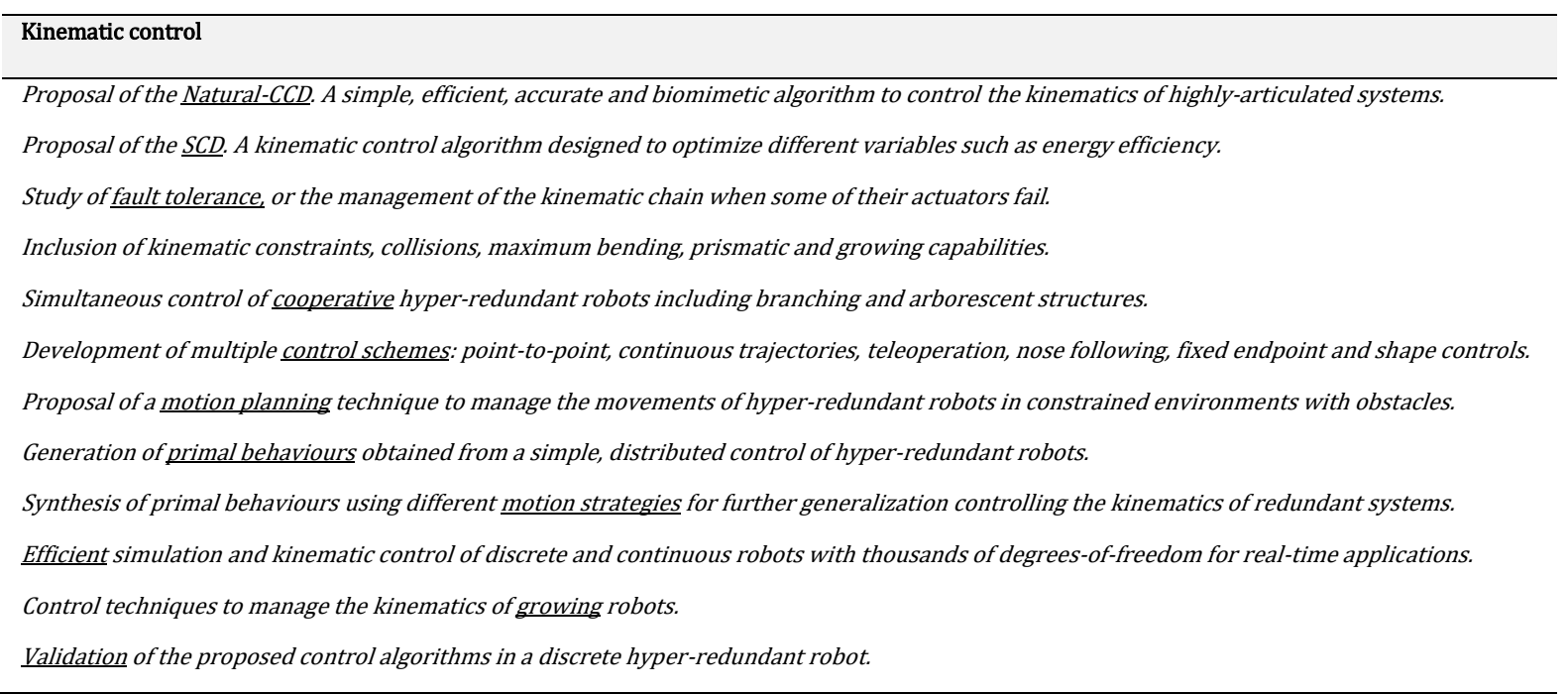

Table 8.4. Contributions derived from the kinematic control. 
Human-Machine Interfaces

Development of a user-oriented interface to command the movements of highly articulated mechanisms.

High robot customization from their size, number of modules, type of joints, degrees-of-freedom, initial poses, precisions and velocities

Easy communication of the interfaces with MATLAB, Unity3D and TCP/IP.

Development of a Virtual Reality interface using the HTC Vive headset to teleoperate hyper-redundant robots.

Development of an Augmented reality interface using Hololens to teleoperate soft robots.

Table 8.5. Contributions related to human-machine interfaces

\begin{tabular}{l}
\hline Teleoperation \\
\hline Development of a bilateral teleoperation architecture based on a position-position scheme in order to command this kind of robots in real-time. \\
Proposal of novel interaction tools to command the movements of such robots including controllers, hand tracking and voice recognition. \\
Validation of the interfaces and interaction tools using standardized tests to measure efficiency, situational awareness and workload. \\
Application of previous contributions in a real scenario with a hyper-redundant robot inspecting a boiler room.
\end{tabular}

Table 8.6. Contributions derived from the teleoperation of highly-articulated manipulators.

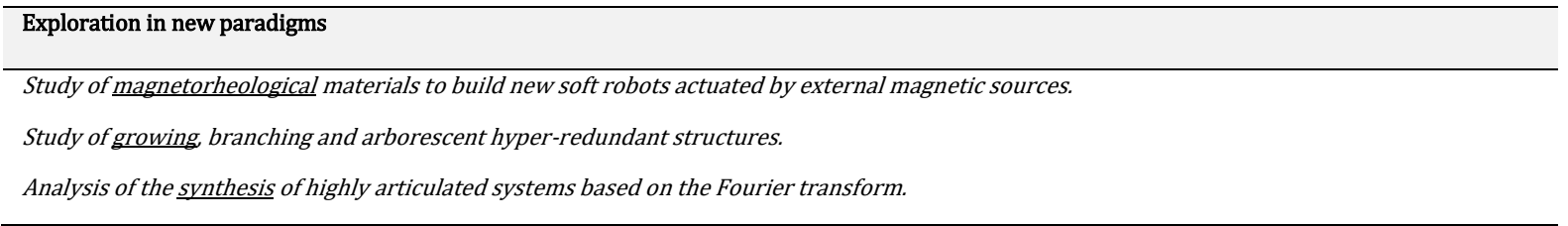

Table 8.7. Contributions derived from the exploration in new paradigms. 


\subsection{Conclusions}

An updated contextualization was firstly proposed to understand the rest of this work. A robot was mathematically described as kinematically hyper-redundant for the first time in the specific literature. This boundary was usually diffuse and this work delineates the limits to represent a very singular change. As a result, it was defined a mechanism as hyper-redundant when has, at least, the double number of degrees of freedom than the minimum required to position and orient its end-effector within a given task-space. Hyper-redundant robots have the ability to provide solutions with dexterity even with the same fault tolerance as the dimension of the task-space. An original and very complete classification was also proposed and updated according to their functionality, number of joints and degrees-of-freedom, actuation and materials.

Then, following the scientific methodology, the challenges of these robots were listed following a thorough state-of-the-art review. Some of the most important are the design, modelling, control and teleoperation, all of them analysed in this work.

Multiple design efforts are proposed inspired by a complete study of previous ones in the scientific literature. These include a discrete hyper-redundant manipulator called Mach, a continuous one called Magno, and a soft robot called Kyma. In all of them, it can be concluded that it is necessary to find a compromise between multiple requirements depending on each application. However, it is possible to optimize such balance and obtain good designs with the available technology, materials and resources. Specifically, the form factor is crucial for the design of soft robots, where the consistency of the materials should be bonded with the force exerted by the actuators in order to obtain the desired behaviour.

It is also deduced from multiple prototypes that a distributed location scheme for the actuators throughout the robot body is typically unfeasible for redundant robots. Therefore, ideas based on external actuation such as those based on tendons, pneumatics or magnetic sources are usually recommended for in-earth applications. They can allow lighter results and ease miniaturization processes. Regarding the latter, it is possible to use magnetic sources as a mean to obtain clever designs such as passive damping mechanisms or remote, contactless actuation.

About the modelling process, it can be concluded that discrete and stiff hyper-redundant robots are relatively easy to model following rigid-body mechanics. However, continuous and, especially, soft robots present movements with noticeable non-linearities, difficult to predict by using traditional methods. These compliant devices are typically designed to interact with their environment and multiple previous works were found to neglect these reciprocities. Thus, some techniques have been proposed to overcome this problem. Firstly, a hybrid method was developed as a combination of a modelling strategy, the Piecewise Constant Curvature model, with an online correction from an on-board array of Inertial Measurement Units. Experimental tests and results showed higher accuracies, including the interactions of the robot with its surroundings compared to previous and similar techniques from the state-of-the-art.

However, the method that is better supported to become the most complete in order to model continuous and soft robots is the real-time Finite Element Method. It is the only strategy that acknowledges both the effect of the morphology and the robot-object interactions. However, this kind of methods are well-known to have high computational costs. This work presents a novel comparison of two techniques to make this 
approach feasible for real-time applications: a heuristic one and the Model Order Reduction. Experiments showed that both of them provided real-time models with relative low errors for a very challenging-tomodel soft robot. In addition, a brief study of different robot-object interactions and compliance was originally presented.

Regarding the control of hyper-redundant, continuous and soft robots, they are mechanically underdetermined so governing their movements can be quite challenging. In other words, a large number of degrees-of-freedom provides an infinite number of solutions to satisfy a given problem. An original strategy entitled as Natural-CCD was initially proposed to solve the inverse kinematics of such robots. This algorithm cyclically and uniformly moves all the degrees-of-freedom by small increments, providing solutions that are kinematically connected to common shapes in nature such as the Fibonacci spiral. This algorithm was found to be very efficient while also providing coherent intermediate configurations in realtime compared to other state-of-the-art approaches.

However, the solution to the inverse kinematics was found to be just part of something much bigger. The Natural-CCD algorithm uses 3 relevant positions to obtain the desired rotation at every control point in the kinematic chain. Interestingly, it was found that different combinations of these positions led to, at least, 40 primal behaviours of highly-articulated systems. The appearance of these behaviours is not consequence of complex assumptions but rather a result of very simple and ellegant mathematical principles. Some of these emerging behaviours include different ways of attraction, repulsion, surrounding, straightening, folding or circumnutations. The initial solution for the inverse kinematics was found to be a specific way of the attraction behaviour of the robot's end-effector towards a desired target. Therefore, a new framework was found to unify the underlying kinematics of highly articulated structures, and a set of motion strategies was also proposed to manage the obtained behaviours. As a result, multiple control methods were designed, not just the inverse kinematics, but also shape controls, path following or follow-the-leader strategies among many others showing high-end performances.

In addition, a very clean and intuitive interface was also developed as open-source with high flexibility and efficiency for real-time applications. The customization options include the robot dimensions, morphology and the initial conditions, number of modules and degrees-of-freedom per module, definition of both prismatic and rotational ones, maximum bending angles and fault-tolerance options, management of self and external collisions, development of cooperative hyper-redundant robots and even the possibility to add growing, branching and arborescent capabilities. Thus, this application can constitute a very complete solution for future researchers to provide additional and valuable contributions to this field.

The presented framework and control algorithms were used and tested in a real hyper-redundant robot and in a real scenario. This robot, Mach, was controlled from the actuators to high-level strategies based on the management of the whole kinematic chain. In contrast to traditional robotics with the actuators distributed in the robot body, the selected remote actuation scheme forced to calculate an intermediate control step to link the output of the actuators to the joints. As a consequence, the robot was controlled in real-time with high flexibility and accuracy in a very elegant and harmonious way as expected from simulation results.

On the other hand, the teleoperation of hyper-redundant, continuous and soft robots is crucial since they are usually devised to work in delicate or hazardous environments. So strategies aimed to manage humanrobot interactions from remote distances are very interesting in this field. Hyper-redundant manipulators have a lot of degrees of freedom, so their spatial understanding is often very difficult to understand 
A bilateral architecture based on a position-position scheme was selected as the most adequate to teleoperate hyper-redundant robot applications by using immersive technologies. This is because the master-slave size relation is similar, there are not strict limitations in the slave speed, a high level of telepresence is required, and the task involves a high number of degrees of freedom.

Experiments with 50 participants were made to contrast 2 hypotheses. On one hand, this work aimed to solve whether immersive 3D interfaces are better or worse than conventional 2D ones in order to teleoperate hyper-redundant robots. On the other hand, some interaction tools were tested by the same population in order to determine which strategy is better suited for the same purpose.

Results showed that immersive interfaces exhibited 33.55\% mean higher efficiency in terms of speed and accuracy and $73.71 \%$ mean higher situational awareness than conventional ones. As a result, immersive interfaces were chosen to teleoperate this kind of robots by $94 \%$ of the participants.

In addition, a total of 6 interaction tools were developed: controller, master-slave, local gestures, remote gestures, voice commands and gestures and voice. Accordingly, efficiency was found indistinguishably the best for both local gestures and gestures and voice, with mean values of around $90 \%$. Also, a workload test showed that local gestures, controller, gestures and voice and remote gestures have half of the workload than the other two approaches. The participants were very accurate in evaluating their performance, which was found to be highly influenced by the level of frustration. Moreover, results showed that naturallanguage tools are more intuitive since exhibited higher performance despite the less previous experience. In fact, $92.31 \%$ selected an interaction tool based on natural language against a conventional one. Specifically, more than half of the users chose the gestures and voice strategy as the preferred one. This is understandable since human beings are used to communicate by simultaneously gesticulating and speaking.

Conducted experiments with a real hyper-redundant robot for inspection tasks in a boiler room showed the efficiency and flexibility of immersive technologies and natural language to perform a remote teleoperation. Both the boiler room and the robot were replicated in a virtual world, where the user could use the bare hands to interact with the virtual robot and command the movements of the real one. Many sensors were used for this application from encoders, thermal and RGB cameras, a head-mounted display with 6 DoF or a hand-tracking visual system. Making an appropriate selection of these sensors is crucial to obtain an optimal teleoperation performance. Consequently, the operators experienced high situational awareness and performed with high efficiency from a remote and safe location. Results from the inspection task revealed that the boiler was functioning adequately whereas application of thermal insulation was recommended in certain locations to prevent future damages. All in all, it can be concluded that immersive technologies and natural language should play an important role in successive approaches towards hyperredundant robots and their teleoperation.

Finally, the repercussion of this work was also analysed since all the previous contributions are vain without a sustainable development perspective. First of all, it is worth highlighting that the highly-articulated robots studied in this document take the traditional ones to their maximum expression, so their applications are uncountable. By increasing the number of degrees-of-freedom in an automatic device, the costs and the complexity may also increase. But these are challenges that will be mitigated with the advance of technology. However, the capabilities of hyper-redundant robots are much higher than those nonredundant since the first ones can always behave like the second ones but not the other way around. These devices are currently playing an important role in our world but future research efforts are always encouraged as Section 8.3 explains. 


\subsection{Future research lines}

As in every scientific enquiry, multiple efforts should be addressed in the future to grant the continuity and progress of the technological advances:

About the design, hyper-redundant, continuous and soft robots are devices which optimization highly relies on multiple parameters that should be adequately tuned because sometimes conflict between each other. So current proliferation of Machine Learning and Evolutionary Algorithms could be promising to optimize the design and construction of such robots based on specific and quantitative requirements. Also, there may be a lot of potential in the use of passive gravity compensation systems to reduce the avalanche effect in the design of this robots, allowing further miniaturization outputs. In addition, synthesis techniques such as those based on biomimicry or the Fourier Transform may also have a lot of potential to provide interesting design solutions. Moreover, magnetorheological materials look very promising to create new ways to design and construct soft robots. These schemes could allow contactless actuation or spring mechanisms to manage their movements from remote locations.

From the modelling process of continuous and soft robots, the use of the Finite Element Method in real-time is highly encouraged. However, the existing software tools such as SOFA need clearer and more intuitive interfaces. Also, there are a lot of specific cases where further optimization is required like using the Model Order Reduction to reduce the dimension of complex morphologies or very thin structures. According to the latter, research on shell elements may also be interesting. But what may be even more relevant is the current techniques focused on the inclusion of a continuously diffused sensory system within the soft materials. Accurate results from these emergent and portable technologies may lead to an unnecessary use of modelling strategies to predict their movements.

About the control of highly-articulated robots, a new framework has been proposed in this work with multiple primal behaviours that, appropriately managed with different motion strategies, could lead to an uncountable number of control architectures. Therefore, new behaviours and movements may emerge if those variables are further explored or if different combinations of such behaviours are proposed. Some of these seem to exhibit chaotic motion under these circumstances, but they may be useful for certain applications still unexplored. Additionally, the study of motion planning techniques is another challenging topic to control the movements of these robots in unstructured environments with obstacles. The construction of the configuration space is unfeasible when a large number of degrees-of-freedom is involved so other techniques are usually provided in the state-of-the-art. However, all of them lack of completeness so future work regarding this is also motivated.

Regarding the teleoperation, the immersive technologies and natural language were found to be an excellent solution to satisfy the needs of these complex devices. However, this work features 6 interaction tools out of many other possible ones. So future work in this matter should be focused on proposing and classifying new interaction strategies in order to compare and select the most appropriate, depending on the hyperredundant robot specifications and the desired applications. 


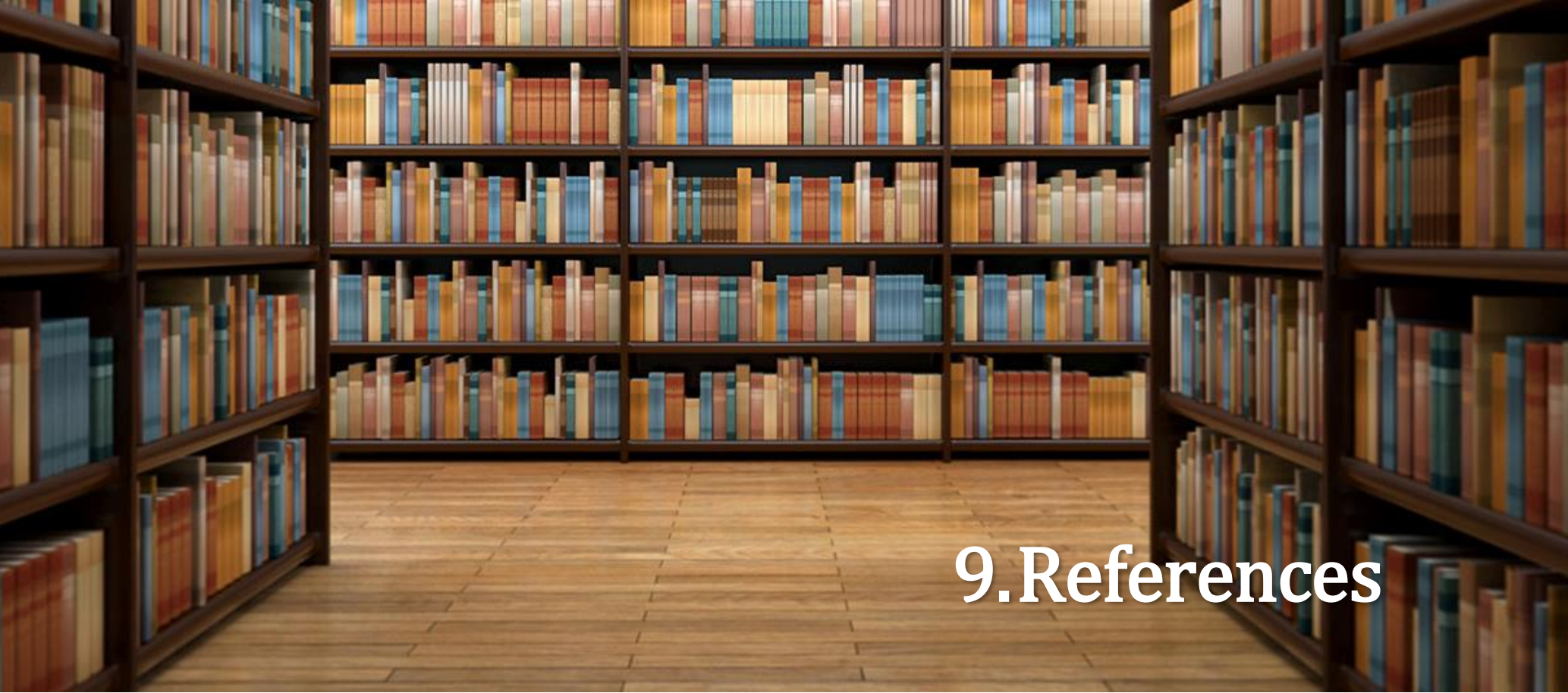

1. Martín-Barrio, A., Robot laparoscópico biomimético e híper-redundante: análisis preliminar de materiales magnéticos, guiado mediante visión artificial y primer estudio de su diseño. Universidad de Valladolid, 2014.

2. Moran, M.E., Evolution of robotic arms. Journal of robotic surgery, 2007. 1(2): p. 103-111.

3. Barrientos, A., et al., Fundamentos de robótica. 2007, Madrid: McGrawHill.

4. Chirikjian, G.S., Theory and applications of hyperredundant robotic manipulators. 1992, California Institute of Technology.

5. Chirikjian, G.S. and J.W. Burdick, A hyper-redundant manipulator. IEEE Robotics \& Automation Magazine, 1994. 1(4): p. 22-9.

6. Kimura, S., et al., A fault-tolerant control algorithm having a decentralized autonomous architecture for space hyper-redundant manipulators. IEEE Transactions on Systems, Man, and Cybernetics-Part A: Systems and Humans, 1998. 28(4): p. 521-527.

7. Martín-Barrio, A., et al., Hyper-Redundant Robots: Classification, State-of-the-Art and Issues. Revista Iberoamericana de Automática e Informática Industrial, 2018. 15(4): p. 351-362.

8. Rossiter, J. and H. Hauser, Soft robotics-the next industrial revolution. IEEE Robot. Autom. Mag, 2016. 23(3): p. 17-20.

9. Duriez, C. Control of elastic soft robots based on realtime finite element method. in Robotics and Automation (ICRA), 2013 IEEE International Conference on. 2013. IEEE.

10. Braganza, D., et al., A neural network controller for continuum robots. IEEE transactions on robotics, 2007. 23(6): p. 1270-1277.
11. Martín-Barrio, A., A. Barrientos, and J. del Cerro, The Natural-CCD algorithm: A novel method to control hyper-redundant and soft robots. Soft Robotics, 2018. 5(3): p. 242-257.

12. Choset, H. and W. Henning, A follow-the-leader approach to serpentine robot motion planning. Journal of Aerospace Engineering, 1999. 12(2): p. 6573.

13. Kobayashi, H. and S. Ohtake. Shape control of hyper redundant manipulator. in Proceedings of 1995 IEEE International Conference on Robotics and Automation. 1995. IEEE.

14. Aracil, R., et al., The human role in telerobotics., in Advances in Telerobotics. 2007, Springer. p. 11-24.

15. Martín-Barrio, A., et al., Application of immersive technologies and natural language to hyperredundant robot teleoperation. Virtual Reality, 2019: p. 1-15.

16. Rheingold, H., Virtual Reality: Exploring the Brave New Technologies of Artificial Experience and Interactive Worlds-From Cyberspace to Teledildonics. 1991, USA: Secker \& Warburg.

17. Vertut, J., Teleoperation and robotics: applications and technology. Vol. 3. 2013, France: Springer Science \& Business Media.

18. Roldán, J.J., et al., Multi-robot Systems, Virtual Reality and ROS: Developing a New Generation of Operator Interfaces., in Robot Operating System (ROS). 2019, Springer. p. 29-64.

19. Hermann, M., T. Pentek, and B. Otto. Design principles for industrie 4.0 scenarios. in 2016 49th Hawaii international conference on system sciences (HICSS). 2016. IEEE. 
20. Trivedi, D., et al., Soft robotics: Biological inspiration, state of the art, and future research. Applied Bionics and Biomechanics, 2008. 5(3): p. 99-117.

21. Hannan, M. and I. Walker. The'elephant trunk'manipulator, design and implementation. in Advanced Intelligent Mechatronics, 2001. Proceedings. 2001 IEEE/ASME International Conference on. 2001. IEEE.

22. Yu, S., et al. An amphibious snake-like robot with terrestrial and aquatic gaits. in Robotics and Automation (ICRA), 2011 IEEE International Conference on. 2011. IEEE.

23. Sujan, V.A. and S. Dubowsky, Design of a lightweight hyper-redundant deployable binary manipulator. Journal of Mechanical Design, 2004. 126(1): p. 29-39.

24. Wright, C., et al. Design and architecture of the unified modular snake robot. in Robotics and Automation (ICRA), 2012 IEEE International Conference on. 2012. IEEE.

25. Bernal, J., R. Flowers-Cano, and A. CarbajalDominguez, Exact calculation of the number of degrees of freedom of a rigid body composed of $n$ particles. Revista mexicana de física E, 2009.55(2): p. 191-195.

26. Del Dottore, E., et al., Toward growing robots: a historical evolution from cellular to plant-inspired robotics. Frontiers in Robotics and AI, 2018. 5: p. 16.

27. Mazzolai, B., Plant-inspired growing robots, in Soft Robotics: Trends, Applications and Challenges. 2017, Springer. p. 57-63.

28. Coad, M.M., et al., Vine robots: Design, teleoperation, and deployment for navigation and exploration. arXiv preprint arXiv:1903.00069, 2019.

29. Sadeghi, A., et al., A plant-inspired robot with soft differential bending capabilities. Bioinspir. Biomim, 2016. 12(1): p. 015001

30. Dent, E.W. and F.B. Gertler, Cytoskeletal dynamics and transport in growth cone motility and axon guidance. Neuron, 2003. 40(2): p. 209-227.

31. Zuckerkandl, E., Coelomic pressures in Sipunculus nudus. The Biological bulletin, 1950. 98(2): p. 161173.

32. Cheetham, A.H., L.-A.C. Hayek, and E. Thomsen, Branching structure in arborescent animals: models of relative growth. Journal of Theoretical Biology, 1980. 85(2): p. 335-369.

33. Hawkes, E.W., et al., A soft robot that navigates its environment through growth. Science Robotics, 2017. 2(8): p. eaan3028.

34. Ranzani, T., et al., A bioinspired soft manipulator for minimally invasive surgery. Bioinspiration \& biomimetics, 2015. 10(3): p. 035008.
35. Blumenschein, L.H., et al., A tip-extending soft robot enables reconfigurable and deployable antennas. IEEE Robotics and Automation Letters, 2018. 3(2): p. 949-956.

36. Luong, J., et al. Eversion and retraction of a soft robot towards the exploration of coral reefs. in 2019 2nd IEEE International Conference on Soft Robotics (RoboSoft). 2019. IEEE.

37. Chirikjian, G.S. A continuum approach to hyperredundant manipulator dynamics. in Intelligent Robots and Systems' 93, IROS'93. Proceedings of the 1993 IEEE/RSJ International Conference on. 1993. IEEE.

38. Chirikjian, G.S., Hyper-redundant manipulator dynamics: A continuum approximation. Advanced Robotics, 1994. 9(3): p. 217-243.

39. Chirikjian, G.S., Inverse kinematics of binary manipulators using a continuum model. Journal of intelligent \& robotic systems, 1997. 19(1): p. 5-22.

40. Brown, H.B., et al. Design and control of a secondgeneration hyper-redundant mechanism. in Intelligent Robots and Systems, 2007. IROS 2007. IEEE/RSJ International Conference on. 2007. IEEE.

41. Mehling, J.S., et al. A minimally invasive tendril robot for in-space inspection. in Biomedical Robotics and Biomechatronics, 2006. BioRob 2006. The First IEEE/RAS-EMBS International Conference on. 2006. IEEE.

42. Robinson, G. and J.B.C. Davies. Continuum robots-a state of the art. in Robotics and Automation, 1999. Proceedings. 1999 IEEE International Conference on. 1999. IEEE.

43. Wolf, A., et al. A mobile hyper redundant mechanism for search and rescue tasks. in Intelligent Robots and Systems, 2003.(IROS 2003). Proceedings. 2003 IEEE/RSJ International Conference on. 2003. IEEE.

44. Ning, K. and F. Wörgötter, A novel concept for building a hyper-redundant chain robot. IEEE transactions on robotics, 2009. 25(6): p. 1237-1248.

45. Sujan, V.A., M.D. Lichter, and S. Dubowsky. Lightweight hyper-redundant binary elements for planetary exploration robots. in Advanced Intelligent Mechatronics, 2001. Proceedings. 2001 IEEE/ASME International Conference on. 2001. IEEE.

46. Ikuta, K., M. Nokata, and S. Aritomi. Biomedical micro robots driven by miniature cybernetic actuator. in Micro Electro Mechanical Systems, 1994, MEMS'94, Proceedings, IEEE Workshop on. 1994. IEEE.

47. Wingert, A., et al. Hyper-redundant robot manipulators actuated by optimized binary-dielectric polymers. in Smart Structures and Materials 2002: Electroactive Polymer Actuators and Devices (EAPAD). 2002. International Society for Optics and Photonics. 
48. Shepherd, R.F., et al., Multigait soft robot. Proceedings of the National Academy of Sciences, 2011. 108(51): p. 20400-20403.

49. Hunter, I.W., J.M. Hollerbach, and J. Ballantyne, $A$ comparative analysis of actuator technologies for robotics. Robotics Review, 1991. 2: p. 299-342.

50. Tedrake, R., Underactuated Robotics: Learning, Planning, and Control for Efficient and Agile Machines: Course Notes for MIT 6.832. Working draft edition, 2009: p. 3.

51. Sánchez-Alonso, R.E., et al., Análisis cinemático de un novedoso robot paralelo reconfigurable. Revista Iberoamericana de Automática e Informática Industrial RIAI, 2016. 13(2): p. 247-257.

52. Grissom, M.D., et al. Design and experimental testing of the OctArm soft robot manipulator. in Unmanned Systems Technology VIII. 2006. International Society for Optics and Photonics.

53. Neppalli, S., et al., Closed-form inverse kinematics for continuum manipulators. Advanced Robotics, 2009. 23(15): p. 2077-2091.

54. Majidi, C., Soft robotics: a perspective-current trends and prospects for the future. Soft Robotics, 2014. 1(1): p. 5-11.

55. Larson, K., Can You Estimate Modulus From Durometer Hardness for Silicones. Dow Corning Corporation, 2016.

56. Bicchi, A. and G. Tonietti, Fast and" soft-arm" tactics [robot arm design]. IEEE Robotics \& Automation Magazine, 2004. 11(2): p. 22-33.

57. Zinn, M., et al., Playing it safe [human-friendly robots]. IEEE Robotics \& Automation Magazine, 2004. 11(2): p. $12-21$.

58. Echávarri, J., et al., Towards a safety index for assessing head injury potential in service robotics. Advanced Robotics, 2013. 27(11): p. 831-844.

59. Marques, L., et al., 3D hyper-redundant robot, in 11th Spanish Portuguese Conference on Electrical Engineering. 2009: Zaragoza, Spain.

60. Webster III, R.J. and B.A. Jones, Design and kinematic modeling of constant curvature continuum robots: $A$ review. The International Journal of Robotics Research, 2010. 29(13): p. 1661-1683.

61. Croom, J.M., et al. Visual sensing of continuum robot shape using self-organizing maps. in Robotics and Automation (ICRA), 2010 IEEE International Conference on. 2010. IEEE.

62. Kang, B.-S. and E.J. Park. Modeling and control of an intrinsic continuum robot actuated by pneumatic artificial muscles. in Advanced Intelligent Mechatronics (AIM), 2016 IEEE International Conference on. 2016. IEEE.
63. Trivedi, D. and C.D. Rahn. Shape sensing for soft robotic manipulators. in ASME 2009 International Design Engineering Technical Conferences and Computers and Information in Engineering Conference. 2009. American Society of Mechanical Engineers Digital Collection.

64. Larkin, D.Q. and D.C. Shafer, Robotic surgery system including position sensors using fiber bragg gratings. 2011, Google Patents.

65. Galloway, K.C., et al., Fiber optic shape sensing for soft robotics. Soft robotics, 2019. 6(5): p. 671-684.

66. Xu, K. and N. Simaan, An investigation of the intrinsic force sensing capabilities of continuum robots. IEEE Transactions on Robotics, 2008. 24(3): p. 576-587.

67. Webster III, R.J., J.M. Romano, and N.J. Cowan, Mechanics of precurved-tube continuum robots. IEEE Transactions on Robotics, 2009. 25(1): p. 67-78.

68. Wehner, M., et al., An integrated design and fabrication strategy for entirely soft, autonomous robots. Nature, 2016. 536(7617): p. 451-455.

69. Rucker, D.C., et al., Equilibrium conformations of concentric-tube continuum robots. The International journal of robotics research, 2010. 29(10): p. 12631280

70. Trivedi, D., A. Lotfi, and C.D. Rahn, Geometrically exact models for soft robotic manipulators. IEEE Transactions on Robotics, 2008. 24(4): p. 773-780.

71. Cheng, L., Z.-G. Hou, and M. Tan, Adaptive neural network tracking control for manipulators with uncertain kinematics, dynamics and actuator model. Automatica, 2009. 45(10): p. 2312-2318

72. Qi, P., et al., Kinematic control of continuum manipulators using a fuzzy-model-based approach. IEEE Transactions on Industrial Electronics, 2016. 63(8): p. 5022-5035.

73. Lozano-Perez, T., Spatial planning: A configuration space approach. IEEE transactions on computers, 1983. $100(2):$ p. $108-120$

74. Dasgupta, B., A. Gupta, and E. Singla, $A$ variational approach to path planning for hyper-redundant manipulators. Robotics and Autonomous Systems, 2009. 57(2): p. 194-201.

75. Shkolnik, A. and R. Tedrake. Path planning in 1000+ dimensions using a task-space Voronoi bias. in Robotics and Automation, 2009. ICRA'09. IEEE International Conference on. 2009. IEEE.

76. Yahya, S., et al. Motion planning of hyper redundant manipulators based on a new geometrical method. in Industrial Technology, 2009. ICIT 2009. IEEE International Conference on. 2009. IEEE.

77. Lee, K., et al. An RRT* path planning for kinematically constrained hyper-redundant inpipe robot. in 
Ubiquitous Robots and Ambient Intelligence (URAI), 2015 12th International Conference on. 2015. IEEE.

78. Collins, T. and W.-M. Shen, PASO: An Integrated Scalable PSO-based Optimization Framework for Hyper-Redundant Manipulator Path Planning and Inverse Kinematics. Information Sciences Institute Technical Report, 2016.

79. Taherifar, A., et al. Path planning for a hyperredundant manipulator with lockable joints using PSO. in Robotics and Mechatronics (ICRoM), 2013 First RSI/ISM International Conference on. 2013. IEEE.

80. Lyons, L.A., R.J. Webster, and R. Alterovitz. Motion planning for active cannulas. in Intelligent Robots and Systems, 2009. IROS 2009. IEEE/RSJ International Conference on. 2009. IEEE.

81. Lamiraux, F. and L.E. Kavraki, Planning paths for elastic objects under manipulation constraints. The International Journal of Robotics Research, 2001. 20(3): p. 188-208.

82. Conru, A.B. A genetic approach to the cable harness routing problem. in Evolutionary Computation, 1994. IEEE World Congress on Computational Intelligence., Proceedings of the First IEEE Conference on. 1994. IEEE.

83. Paul, R.P., Robot manipulators: mathematics, programming, and control: the computer control of robot manipulators. 1981: Richard Paul.

84. Conkur, E.S. and R. Buckingham, Clarifying the definition of redundancy as used in robotics. Robotica, 1997. 15(5): p. 583-586.

85. Espinoza, M.S., et al. Inverse Kinematics of a 10 DOF Modular Hyper-Redundant Robot Resorting to Exhaustive and Error-Optimization Methods: $A$ Comparative Study. in Robotics Symposium and Latin American Robotics Symposium (SBR-LARS), 2012 Brazilian. 2012. IEEE.

86. Gravagne, I.A., C.D. Rahn, and I.D. Walker. Good vibrations: a vibration damping setpoint controller for continuum robots. in Robotics and Automation, 2001. Proceedings 2001 ICRA. IEEE International Conference on. 2001. IEEE.

87. Sheridan, T.B., Telerobotics, automation, and human supervisory control. 1992: MIT press.

88. Vincent, J.F., et al., Biomimetics: its practice and theory. Journal of the Royal Society Interface, 2006. 3(9): p. 471-482.

89. Turner, J.S. and R.C. Soar. Beyond biomimicry: What termites can tell us about realizing the living building. in First International Conference on Industrialized, Intelligent Construction at Loughborough University. 2008 .
90. Johnson, E.R., Reinventing biological life, reinventing'the human'. Ephemera: theory and politics in organization., 2010.10(2): p. 177-193.

91. Crespi, A., et al., AmphiBot I: an amphibious snake-like robot. Robotics and Autonomous Systems, 2005. 50(4): p. 163-175.

92. Van Leeuwen, J. and W.M. Kier, Functional design of tentacles in squid: linking sarcomere ultrastructure to gross morphological dynamics. Philosophical Transactions of the Royal Society of London B: Biological Sciences, 1997. 352(1353): p. 551-571.

93. Yekutieli, Y., et al., Dynamic model of the octopus arm. I. Biomechanics of the octopus reaching movement. Journal of neurophysiology, 2005. 94(2): p. 14431458.

94. Laschi, C., et al., Soft robot arm inspired by the octopus. Advanced Robotics, 2012. 26(7): p. 709-727.

95. Wilson, J., et al., Flexible robot manipulators and grippers: Relatives of elephant trunks and squid tentacles. Robots and Biological Systems: Towards a New Bionics?, 1993: p. 475-494.

96. OC-Robotics. Series II, X125 System. 2015 [http://www.ocrobotics.com/technology-/series-iix125-system/] -October 2017]

97. Yang, Y. and W. Zhang. An elephant-trunk manipulator with twisting flexional rods. in Robotics and Biomimetics (ROBIO), 2015 IEEE International Conference on. 2015. IEEE.

98. Dong, X., et al., Development of a slender continuum robotic system for on-wing inspection/repair of gas turbine engines. Robotics and Computer-Integrated Manufacturing, 2017. 44: p. 218-229.

99. McMahan, W., B.A. Jones, and I.D. Walker. Design and implementation of a multi-section continuum robot: Air-Octor. in Intelligent Robots and Systems, 2005.(IROS 2005). 2005 IEEE/RSJ International Conference on 2005. IEEE.

100. Trivedi, D., A. Lotfi, and C.D. Rahn. Geometrically exact dynamic models for soft robotic manipulators. in Intelligent Robots and Systems, 2007. IROS 2007. IEEE/RSJ International Conference on. 2007. IEEE.

101. Rolf, M. and J.J. Steil. Constant curvature continuum kinematics as fast approximate model for the Bionic Handling Assistant. in Intelligent Robots and Systems (IROS), 2012 IEEE/RSI International Conference on. 2012. IEEE.

102. Calisti, M., et al., An octopus-bioinspired solution to movement and manipulation for soft robots. Bioinspiration \& biomimetics, 2011. 6(3): p. 036002

103. Hirose, S. and M. Mori. Biologically inspired snake-like robots in Robotics and Biomimetics, 2004. ROBIO 2004. IEEE International Conference on. 2004. IEEE. 
104. Worst, R. and R. Linnemann. Construction and operation of a snake-like robot in Intelligence and Systems, 1996., IEEE International Joint Symposia on. 1996. IEEE

105. Klaassen, B. and K.L. Paap. GMD-SNAKE2: a snake-like robot driven by wheels and a method for motion control. in Robotics and Automation, 1999. Proceedings. 1999 IEEE International Conference on. 1999. IEEE.

106. Streich, H. and O. Adria. Software approach for the autonomous inspection robot MAKRO. in Robotics and Automation, 2004. Proceedings. ICRA'04. 2004 IEEE International Conference on. 2004. IEEE.

107. Kimura, H. and S. Hirose. Development of Genbu: Active wheel passive joint articulated mobile robot. in Intelligent Robots and Systems, 2002. IEEE/RS/ International Conference on. 2002. IEEE

108. Osuka, K. and H. Kitajima. Development of mobile inspection robot for rescue activities: MOIRA. in Intelligent Robots and Systems, 2003.(IROS 2003). Proceedings. 2003 IEEE/RSJ International Conference on. 2003. IEEE.

109. Kamegawa, T., et al. Development of the snake-like rescue robot" kohga". in Robotics and Automation, 2004. Proceedings. ICRA'04. 2004 IEEE International Conference on. 2004. IEEE.

110. Osuka, K. On Development of Snake-like Robots to Search a Victim Left inside Debris. in Robotics and Biomimetics, 2004. ROBIO 2004. IEEE International Conference on. 2004. IEEE.

111. Granosik, G., M.G. Hansen, and J. Borenstein, The OmniTread serpentine robot for industrial inspection and surveillance. Industrial Robot: An International Journal, 2005. 32(2): p. 139-148.

112. Crespi, A. and A.J. Ijspeert. AmphiBot II: An amphibious snake robot that crawls and swims using a central pattern generator. in Proceedings of the 9th international conference on climbing and walking robots (CLAWAR 2006). 2006.

113. Liljeback, P., O. Stavdahl, and A. Beitnes. SnakeFighter-development of a water hydraulic fire fighting snake robot. in Control, Automation, Robotics and Vision, 2006. ICARCV'06. 9th International Conference on. 2006. IEEE.

114. Ohno, H. and S. Hirose. Design of slim slime robot and its gait of locomotion. in Intelligent Robots and Systems, 2001. Proceedings. 2001 IEEE/RSJ International Conference on. 2001. IEEE.

115. Liljeback, P., et al., Snake robot locomotion in environments with obstacles. IEEE/ASME Transactions on Mechatronics, 2012. 17(6): p. 11581169.

116. Long, G., J. Anderson, and J. Borenstein. The kinematic design of the omnipede: a new approach to obstacle traversion. in Robotics and Automation, 2002
Proceedings. ICRA'02. IEEE International Conference on. 2002. IEEE.

117. Liljebäck, P., et al., A review on modelling, implementation, and control of snake robots. Robotics and Autonomous Systems, 2012. 60(1): p. 29-40.

118. Maity, A. and S. Majumder. Serpentine robots: A study of design philosophy. in Advanced Robotics (ICAR), 2011 15th International Conference on. 2011. IEEE.

119. Transeth, A.A., K.Y. Pettersen, and P. Liljebäck, $A$ survey on snake robot modeling and locomotion. Robotica, 2009. 27(7): p. 999-1015.

120. Ikeda, H. and N. Takanashi, Joint assembly movable like a human arm. 1987, Google Patents.

121. Paljug, E., T. Ohm, and S. Hayati. The JPL serpentine robot: a 12-DOF system for inspection. in Robotics and Automation, 1995. Proceedings., 1995 IEEE International Conference on. 1995. IEEE.

122. Buckingham, R. and A. Graham, Nuclear snake-arm robots. Industrial Robot: An International Journal, 2012. 39(1): p. 6-11.

123. Walker, I.D., Continuous backbone "continuum" robot manipulators. ISRN Robotics, 2013. 2013.

124. Chirikjian, G.S. and J.W. Burdick, Kinematically optimal hyper-redundant manipulator configurations. IEEE transactions on Robotics and Automation, 1995. 11(6): p. 794-806.

125. Chirikjian, G.S. and J.W. Burdick. Design and Experiments with a 30 DOF Robot. in Robotics and Automation, 1993. Proceedings., 1993 IEEE International Conference on. 1993. IEEE.

126. Hannan, M.W. and I.D. Walker, Kinematics and the implementation of an elephant's trunk manipulator and other continuum style robots. Journal of Field Robotics, 2003. 20(2): p. 45-63.

127. Cowan, L.S. and I.D. Walker, The importance of continuous and discrete elements in continuum robots. International Journal of Advanced Robotic Systems, 2013. 10(3): p. 165

128. Ikuta, K., T. Hasegawa, and S. Daifu. Hyper redundant miniature manipulator" Hyper Finger" for remote minimally invasive surgery in deep area. in Robotics and Automation, 2003. Proceedings. ICRA'03. IEEE International Conference on. 2003. IEEE.

129. Mahvash, M. and P.E. Dupont, Stiffness control of surgical continuum manipulators. IEEE Transactions on Robotics, 2011. 27(2): p. 334-345

130. Nickl, M., S. Jörg, and G. Hirzinger, The virtual path: The domain model for the design of the MIRO surgical robotic system. IFAC Proceedings Volumes, 2009. 42(16): p. 1-7 
131. Sareh, S., et al. A $7.5 \mathrm{~mm}$ Steiner chain fibre-optic system for multi-segment flex sensing. in Intelligent Robots and Systems (IROS), 2015 IEEE/RS) International Conference on. 2015. IEEE.

132. Kwok, K.-W., et al., Dimensionality reduction in controlling articulated snake robot for endoscopy under dynamic active constraints. IEEE Transactions on Robotics, 2013. 29(1): p. 15-31.

133. Slatkin, A.B., J. Burdick, and W. Grundfest. The development of a robotic endoscope. in Intelligent Robots and Systems 95. 'Human Robot Interaction and Cooperative Robots', Proceedings. 1995 IEEE/RSJ International Conference on. 1995. IEEE.

134. Chen, G., M.T. Pham, and T. Redarce, Sensor-based guidance control of a continuum robot for a semiautonomous colonoscopy. Robotics and autonomous systems, 2009. 57(6): p. 712-722.

135. Paek, J., I. Cho, and J. Kim, Microrobotic tentacles with spiral bending capability based on shape-engineered elastomeric microtubes. Scientific reports, 2015. 5: p. 10768.

136. Stokes, A.A., et al., A hybrid combining hard and soft robots. Soft Robotics, 2014. 1(1): p. 70-74

137. Li, Z. and R. Du, Design and analysis of a bio-inspired wire-driven multi-section flexible robot. International Journal of Advanced Robotic Systems, 2013. 10(4): p. 209

138. Yang, Y. and W. Zhang. ET Arm: Highly Compliant Elephant-Trunk Continuum Manipulator. in International Conference on Intelligent Robotics and Applications. 2014. Springer.

139. Immega, G. and K. Antonelli. The KSI tentacle manipulator. in Robotics and Automation, 1995. Proceedings., 1995 IEEE International Conference on. 1995. IEEE.

140. McMahan, W., et al., Robotic manipulators inspired by cephalopod limbs. Proceedings of the Canadian Engineering Education Association, 2011.

141. Neppalli, S. and B.A. Jones. Design, construction, and analysis of a continuum robot. in Intelligent Robots and Systems, 2007. IROS 2007. IEEE/RSJ International Conference on. 2007. IEEE.

142. Mahl, T., A. Hildebrandt, and 0. Sawodny. Forward kinematics of a compliant pneumatically actuated redundant manipulator. in Industrial Electronics and Applications (ICIEA), 2012 7th IEEE Conference on. 2012. IEEE.

143. Rus, D. and M.T. Tolley, Design, fabrication and control of soft robots. Nature, 2015. 521(7553): p. 467.

144. Martinez, R.V., et al., Robotic tentacles with threedimensional mobility based on flexible elastomers. Advanced Materials, 2013. 25(2): p. 205-212.
145. Kang, R., et al., Design, modeling and control of a pneumatically actuated manipulator inspired by biological continuum structures. Bioinspiration \& biomimetics, 2013. 8(3): p. 036008.

146. Renda, F., et al., A 3D steady-state model of a tendondriven continuum soft manipulator inspired by the octopus arm. Bioinspiration \& biomimetics, 2012. 7(2): p. 025006

147. Cheng, N.G., et al. Design and analysis of a robust, lowcost, highly articulated manipulator enabled by jamming of granular media. in Robotics and Automation (ICRA), 2012 IEEE International Conference on. 2012. IEEE.

148. Nemitz, M.P., et al., Using voice coils to actuate modular soft robots: wormbot, an example. Soft robotics, 2016. 3(4): p. 198-204.

149. Seok, S., et al., Meshworm: a peristaltic soft robot with antagonistic nickel titanium coil actuators. IEEE/ASME Transactions on mechatronics, 2013. 18(5): p. $1485-1497$.

150. Mutlu, R., G. Alici, and W. Li, An effective methodology to solve inverse kinematics of electroactive polymer actuators modelled as active and soft robotic structures. Mechanism and Machine Theory, 2013. 67: p. $94-110$

151. Laschi, C. and M. Cianchetti, Soft robotics: new perspectives for robot bodyware and control. Frontiers in bioengineering and biotechnology, 2014.

152. Marchese, A.D., C.D. Onal, and D. Rus. Soft robot actuators using energy-efficient valves controlled by electropermanent magnets. in Intelligent Robots and Systems (IROS), 2011 IEEE/RSJ International Conference on. 2011. IEEE.

153. Mosadegh, B., et al., Pneumatic networks for soft robotics that actuate rapidly. Advanced Functional Materials, 2014. 24(15): p. 2163-2170.

154. Shepherd, R.F., et al., Using explosions to power a soft robot. Angewandte Chemie, 2013. 125(10): p. 29642968.

155. Suzumori, K., et al. A bending pneumatic rubber actuator realizing soft-bodied manta swimming robot. in Robotics and Automation, 2007 IEEE International Conference on. 2007. IEEE.

156. Jung, K., et al., Artificial annelid robot driven by soft actuators. Bioinspiration \& biomimetics, 2007. 2(2): p. S42.

157. Lin, H.-T., G.G. Leisk, and B. Trimmer, GoQBot: $a$ caterpillar-inspired soft-bodied rolling robot. Bioinspiration \& biomimetics, 2011. 6(2): p. 026007.

158. Kim, S., C. Laschi, and B. Trimmer, Soft robotics: a bioinspired evolution in robotics. Trends in biotechnology, 2013. 31(5): p. 287-294. 
159. Best, C.M., et al., A new soft robot control method: Using model predictive control for a pneumatically actuated humanoid. IEEE Robotics \& Automation Magazine, 2016. 23(3): p. 75-84

160. Morales Bieze, T., Contribution to the kinematic modeling and control of soft manipulators using computational mechanics. 2017, Lille 1.

161. Chirikjian, G.S. and J.W. Burdick, $A$ modal approach to hyper-redundant manipulator kinematics. IEEE Transactions on Robotics and Automation, 1994. 10(3): p. 343-354.

162. Gravagne, I.A. and I.D. Walker. Kinematic transformations for remotely-actuated planar continuum robots. in Robotics and Automation, 2000 Proceedings. ICRA'00. IEEE International Conference on. 2000. IEEE.

163. Jones, B.A. and I.D. Walker, Kinematics for multisection continuum robots. IEEE Transactions on Robotics, 2006. 22(1): p. 43-55.

164. Tatlicioglu, E., I.D. Walker, and D.M. Dawson. Dynamic modelling for planar extensible continuum robot manipulators. in Robotics and Automation, 2007 IEEE International Conference on. 2007. IEEE.

165. George Thuruthel, T., et al., Control Strategies for Soft Robotic Manipulators: A Survey. Soft robotics, 2018. 5(2): p. 149-163

166. Camarillo, D.B., C.R. Carlson, and J.K. Salisbury, Configuration tracking for continuum manipulators with coupled tendon drive. IEEE Transactions on Robotics, 2009. 25(4): p. 798-808.

167. Renda, F., et al., Dynamic model of a multibending soft robot arm driven by cables. IEEE Transactions on Robotics, 2014.30(5): p. 1109-1122.

168. Trivedi, D., D. Dienno, and C.D. Rahn, Optimal, modelbased design of soft robotic manipulators. Journal of Mechanical Design, 2008. 130(9): p. 091402

169. Pai, D.K. Strands: Interactive simulation of thin solids using cosserat models. in Computer Graphics Forum. 2002. Wiley Online Library.

170. Pawlowski, B. and J. Zhao. Modeling of Soft Manipulators with Couplings between Actuations and Body Deformations. in 2018 Annual American Control Conference (ACC). 2018. IEEE.

171. Renda, F., et al., Discrete Cosserat approach for multisection soft manipulator dynamics IEEE Transactions on Robotics, 2018. 34(6): p. 1518-1533.

172. Zheng, T., et al. Dynamic continuum arm model for use with underwater robotic manipulators inspired by octopus vulgaris. in Robotics and Automation (ICRA), 2012 IEEE International Conference on. 2012. IEEE.

173. Zhang, Z., et al. Kinematic modeling and observer based control of soft robot using real-time finite element method. in Intelligent Robots and Systems
(IROS), 2016 IEEE/RSI International Conference on. 2016. IEEE

174. Coevoet, E., et al., Software toolkit for modeling simulation, and control of soft robots. Advanced Robotics, 2017. 31(22): p. 1208-1224.

175. Runge, G., et al. A framework for the kinematic modeling of soft material robots combining finite element analysis and piecewise constant curvature kinematics. in Control, Automation and Robotics (ICCAR), 2017 3rd International Conference on. 2017. IEEE.

176. Grazioso, S., G. Di Gironimo, and B. Siciliano, $A$ Geometrically Exact Model for Soft Continuum Robots: The Finite Element Deformation Space Formulation. Soft robotics, 2018

177. Hu, Y., et al., ChainQueen: A Real-Time Differentiable Physical Simulator for Soft Robotics. arXiv preprint arXiv:1810.01054, 2018.

178. Giorelli, M., et al. A feed-forward neural network learning the inverse kinetics of a soft cable-driven manipulator moving in three-dimensional space. in Intelligent Robots and Systems (IROS), 2013 IEEE/RSJ International Conference on. 2013. IEEE.

179. Giorelli, M., et al., Neural network and jacobian method for solving the inverse statics of a cabledriven soft arm with nonconstant curvature. IEEE Transactions on Robotics, 2015. 31(4): p. 823-834.

180. George Thuruthel, T., et al., Learning closed loop kinematic controllers for continuum manipulators in unstructured environments. Soft robotics, 2017. 4(3): p. 285-296.

181. Thuruthel, T.G., et al., Stable Open Loop Control of Soft Robotic Manipulators. IEEE Robotics and Automation Letters, 2018. 3(2): p. 1292-1298.

182. Lakhal, O., A. Melingui, and R. Merzouki, Hybrid approach for modeling and solving of kinematics of a compact bionic handling assistant manipulator. IEEE/ASME Transactions on Mechatronics, 2016. 21(3): p. 1326-1335

183. Ma, S. and M. Konno. An obstacle avoidance scheme for hyper-redundant manipulators-global motion planning in posture space in Proceedings of International Conference on Robotics and Automation. 1997. IEEE.

184. Lattanzi, D. and G. Miller, Review of robotic infrastructure inspection systems. Journal of Infrastructure Systems, 2017. 23(3): p. 04017004.

185. Montero, R., et al., Past, present and future of robotic tunnel inspection. Automation in Construction, 2015. 59: p. 99-112.

186. Katrasnik, J., F. Pernus, and B. Likar, A survey of mobile robots for distribution power line inspection. IEEE Transactions on power delivery, 2009. 25(1): p. 485-493. 
187. Almadhoun, $\mathrm{R}$, et al., $A$ survey on inspecting structures using robotic systems. International Journal of Advanced Robotic Systems, 2016. 13(6): p. 1729881416663664

188. Fernández-Caramés, T.M., et al., $A$ fog computing based cyber-physical system for the automation of pipe-related tasks in the Industry 4.0 shipyard. Sensors, 2018. 18(6): p. 1961.

189. Kafali, M., M. Ozkok, and S. Cebi, Evaluation of pipe cutting technologies in shipbuilding. Brodogradnja: Teorija i praksa brodogradnje i pomorske tehnike, 2014. 65(2): p. 33-48.

190. Ledezma, F.D., et al. A Market Survey of Offshore Underwater Robotic Inspection Technologies for the Oil and Gas Industry. in SPE Saudi Arabia Section Annual Technical Symposium and Exhibition. 2015. Society of Petroleum Engineers.

191. Botti, L., E. Ferrari, and C. Mora, Automated entry technologies for confined space work activities: $A$ survey. Journal of occupational and environmental hygiene, 2017. 14(4): p. 271-284

192. Hauser, H., Resilient machines through adaptive morphology. Nature Machine Intelligence, 2019. 1(8): p. 338-339.

193. Bongard, J., et al. Material properties affect evolutions ability to exploit morphological computation in growing soft-bodied creatures. in Artificial Life Conference Proceedings 13. 2016. MIT Press.

194. Zhu, Y., J. Rossiter, and H. Hauser. Learning in Growing Robots: Knowledge Transfer from Tadpole to Frog Robot. in Conference on Biomimetic and Biohybrid Systems. 2019. Springer.

195. Tsukagoshi, H., A. Kitagawa, and M. Segawa. Active hose: An artificial elephant's nose with maneuverability for rescue operation. in Proceedings 2001 ICRA. IEEE International Conference on Robotics and Automation (Cat. No. 01CH37164). 2001. IEEE

196. Greer, J.D., et al. Series pneumatic artificial muscles (sPAMs) and application to a soft continuum robot. in 2017 IEEE International Conference on Robotics and Automation (ICRA). 2017. IEEE.

197. Greer, J.D., et al., $A$ soft, steerable continuum robot that grows via tip extension. Soft robotics, 2019. 6(1): p. $95-108$.

198. Greer, J.D., et al. Obstacle-aided navigation of a soft growing robot. in 2018 IEEE International Conference on Robotics and Automation (ICRA). 2018. IEEE.

199. Mazzolai, B., et al., A miniaturized mechatronic system inspired by plant roots for soil exploration. IEEE/ASME Transactions on Mechatronics, 2010. 16(2): p. 201-212.

200. Sadeghi, A., et al. Robotic mechanism for soil penetration inspired by plant root. in 2013 IEEE
International Conference on Robotics and Automation. 2013. IEEE.

201. Sadeghi, A., A. Mondini, and B. Mazzolai, Toward selfgrowing soft robots inspired by plant roots and based on additive manufacturing technologies. Soft robotics, 2017. 4(3): p. 211-223

202. Must, I., E. Sinibaldi, and B. Mazzolai, $A$ variablestiffness tendril-like soft robot based on reversible osmotic actuation. Nature communications, 2019. 10(1): p. $1-8$.

203. Wooten, M.B. and I.D. Walker, Vine-inspired continuum tendril robots and circumnutations. Robotics, 2018. 7(3): p. 58

204. Calisti, M., et al. Design and development of a soft robot with crawling and grasping capabilities. in 2012 IEEE International Conference on Robotics and Automation. 2012. IEEE.

205. Hauser, H., et al., Towards a theoretical foundation for morphological computation with compliant bodies. Biological cybernetics, 2011. 105(5-6): p. 355-370.

206. Wang, T., H. Li, and C. Meng. Collective grasping for non-cooperative objects using modular selfreconfigurable robots. in 2015 IEEE/RSJ International Conference on Intelligent Robots and Systems (IROS). 2015. IEEE.

207. Yan, T., S. Teshigawara, and H.H. Asada. Design of a Growing Robot Inspired by Plant Growth. in 2019 IEEE/RSJ International Conference on Intelligent Robots and Systems (IROS). 2019. IEEE.

208. Chen, J.Y., E.C. Haas, and M.J. Barnes, Human performance issues and user interface design for teleoperated robots. IEEE Transactions on Systems, Man, and Cybernetics, Part C (Applications and Reviews), 2007. 37(6): p. 1231-1245.

209. Hedayati, H., M. Walker, and D. Szafir. Improving collocated robot teleoperation with augmented reality. in Proceedings of the 2018 ACM/IEEE International Conference on Human-Robot Interaction. 2018. ACM.

210. Nielsen, C.W., M.A. Goodrich, and R.W. Ricks, Ecological interfaces for improving mobile robot teleoperation. IEEE Transactions on Robotics, 2007. 23(5): p. 927-941.

211. Cheng-jun, D., et al. Design of mobile robot teleoperation system based on virtual reality. in 2009 IEEE International Conference on Automation and Logistics. 2009. IEEE.

212. Mostefa, M., et al. Design of mobile robot teleoperation system based on virtual reality. in 2015 3rd International Conference on Control, Engineering \& Information Technology (CEIT). 2015. IEEE.

213. Kot, T. and P. Novák, Application of virtual reality in teleoperation of the military mobile robotic system 
TAROS. International Journal of Advanced Robotic Systems, 2018. 15(1): p. 1729881417751545.

214. Li, N., et al. Flying frustum: $A$ spatial interface for enhancing human-uav awareness. in Proceedings of the 3rd International Conference on Human-Agent Interaction. 2015. ACM.

215. Lipton, J.I., A.J. Fay, and D. Rus, Baxter's homunculus: Virtual reality spaces for teleoperation in manufacturing. IEEE Robotics and Automation Letters, 2018. 3(1): p. 179-186

216. Bugalia, N., et al. Immersive environment for robotic tele-operation. in Proceedings of the 2015 Conference on Advances In Robotics. 2015. ACM.

217. Roldán, J.J., et al., A training system for Industry 4.0 operators in complex assemblies based on virtual reality and process mining. Robotics and ComputerIntegrated Manufacturing, 2019. 59: p. 305-316.

218. Labonte, D., P. Boissy, and F. Michaud, Comparative analysis of 3-D robot teleoperation interfaces with novice users. IEEE Transactions on Systems, Man, and Cybernetics, Part B (Cybernetics), 2010. 40(5): p. 1331-1342.

219. Koizumi, S., et al. Preliminary field trial for teleoperated communication robots. in ROMAN2006The 15th IEEE International Symposium on Robot and Human Interactive Communication. 2006. IEEE.

220. Ren, L., et al. A master-slave control system with workspaces isomerism for teleoperation of a snake robot. in 2017 39th annual international conference of the IEEE engineering in medicine and biology society $(E M B C)$. 2017. IEEE.

221. Bhattacherjee, S., et al., Kinematics and Teleoperation of Tendon Driven Continuum Robot. Procedia computer science, 2018. 133: p. 879-886.

222. Kapadia, A.D., I.D. Walker, and E. Tatlicioglu. Teleoperation control of a redundant continuum manipulator using a non-redundant rigid-link master. in 2012 IEEE/RSJ International Conference on Intelligent Robots and Systems. 2012. IEEE.

223. $\mathrm{Hu}, \mathrm{C}$. , et al. Visual gesture recognition for humanmachine interface of robot teleoperation. in Proceedings 2003 IEEE/RSJ International Conference on Intelligent Robots and Systems (IROS 2003)(Cat. No. 03CH37453). 2003. IEEE

224. Mantecón, T., et al. Hand gesture recognition using infrared imagery provided by leap motion controller. in International Conference on Advanced Concepts for Intelligent Vision Systems. 2016. Springer.

225. Frigola, M., J. Fernandez, and J. Aranda. Visual human machine interface by gestures. in 2003 IEEE International Conference on Robotics and Automation (Cat. No. O3CH37422). 2003. IEEE.

226. Kuno, Y., et al. Robotic wheelchair with three control modes. in Proceedings 1999 IEEE International
Conference on Robotics and Automation (Cat. No. 99CH36288C). 1999. IEEE.

227. Mashood, A., et al. A gesture based kinect for quadrotor control. in 2015 International Conference on Information and Communication Technology Research (ICTRC). 2015. IEEE.

228. Stern, H., J. Wachs, and Y. Edan. A method for selection of optimal hand gesture vocabularies. in International Gesture Workshop. 2007. Springer.

229. Shirwalkar, S., et al. Telemanipulation of an industrial robotic arm using gesture recognition with Kinect. in 2013 International Conference on Control, Automation, Robotics and Embedded Systems (CARE). 2013. IEEE

230. Aracil, R., et al., Telerobotic system for live-power line maintenance: ROBTET. Control Engineering Practice, 2002. 10(11): p. 1271-1281.

231. Draper, M., et al. Manual versus speech input for unmanned aerial vehicle control station operations. in Proceedings of the Human Factors and Ergonomics Society Annual Meeting. 2003. SAGE Publications Sage CA: Los Angeles, CA.

232. Rininsland, H., ARTEMIS. A telemanipulator for cardiac surgery. European Journal of Cardio-Thoracic Surgery, 1999. 16(Supplement_2): p. S106-S111.

233. Lu, Y., et al. Voice based control for humanoid teleoperation. in 2010 International Conference on Intelligent System Design and Engineering Application. 2010. IEEE.

234. Poncela, A. and L. Gallardo-Estrella, Command-based voice teleoperation of a mobile robot via a humanrobot interface. Robotica, 2015. 33(1): p. 1-18.

235. Lueth, T.C., et al. KANTRA-human-machine interaction for intelligent robots using natural language. in Proceedings of 1994 3rd IEEE International Workshop on Robot and Human Communication. 1994. IEEE.

236. Yoshizaki, M., Y. Kuno, and A. Nakamura. Humanrobot interface based on the mutual assistance between speech and vision. in Proceedings of the 2001 workshop on Perceptive user interfaces. 2001. ACM.

237. Shilling, R.D. and B. Shinn-Cunningham, Virtual auditory displays. Handbook of virtual environment technology, 2002: p. 65-92.

238. Sadeghi, A., L. Beccai, and B. Mazzolai. Innovative soft robots based on electro-rheological fluids. in 2012 IEEE/RSI International Conference on Intelligent Robots and Systems. 2012. IEEE.

239. Jeon, S., et al., $A$ magnetically controlled soft microrobot steering a guidewire in a threedimensional phantom vascular network. Soft robotics, 2019.6(1): p. 54-68. 
240. Nie, X and V. Krovi, Fourier methods for kinematic synthesis of coupled serial chain mechanisms. J. Mech. Des., 2005. 127(2): p. 232-241.

241. Cianchetti, M., et al., Soft robotics technologies to address shortcomings in today's minimally invasive surgery: the STIFF-FLOP approach. Soft robotics, 2014. 1(2): p. 122-131.

242. Shepherd, R.F., et al., Using explosions to power a soft robot. Angewandte Chemie International Edition, 2013. 52(10): p. 2892-2896.

243. Duckett, T., et al., Agricultural robotics: the future of robotic agriculture arXiv preprint arXiv:1806.06762, 2018

244. Laschi, C., et al., Soft Robotics: Trends, Applications and Challenges. Livorno, Italy, 2016.

245. Young, S.J., Real Time Languages: Design and Development. 1982, USA: Ellis Horwood Ltd.

246. Faure, F., et al., Sofa: A multi-model framework for interactive physical simulation, in Soft tissue biomechanical modeling for computer assisted surgery. 2012, Springer. p. 283-321.

247. Goury, 0. and C. Duriez, Fast, generic, and reliable control and simulation of soft robots using model order reduction. IEEE Transactions on Robotics, 2018. 34(6): p. 1565-1576

248. Katzschmann, R.K., et al. Dynamically Closed-Loop Controlled Soft Robotic Arm using a Reduced Order Finite Element Model with State Observer. in 2019 2nd IEEE International Conference on Soft Robotics (RoboSoft). 2019. IEEE.

249. Sangpradit, K., et al., Finite-element modeling of soft tissue rolling indentation. IEEE Transactions on Biomedical Engineering, 2011. 58(12): p. 3319-3327.

250. Wang, L.-C. and C.-C. Chen, A combined optimization method for solving the inverse kinematics problems of mechanical manipulators. IEEE Transactions on Robotics and Automation, 1991. 7(4): p. 489-499.

251. Mukundan, R., A robust inverse kinematics algorithm for animating a joint chain. International Journal of Computer Applications in Technology, 2009. 34(4): p. 303-308.

252. Aristidou, A. and J. Lasenby, FABRIK: A fast, iterative solver for the Inverse Kinematics problem. Graphical Models, 2011. 73(5): p. 243-260.

253. Loshchilov, I., M. Schoenauer, and M. Sebag. Adaptive coordinate descent. in Proceedings of the 13th annual conference on Genetic and evolutionary computation. 2011. ACM.

254. Wright, S.J., Coordinate descent algorithms. Mathematical Programming, 2015. 151(1): p. 3-34.

255. Hargroves, K. and M. Smith, Innovation inspired by nature: biomimicry. Ecos, 2006. 2006(129): p. 27-29.
256. Lawrence, J.D., A catalog of special plane curves. 2013: Courier Corporation.

257. Conway, J.H. and R.K. Guy, The Book of Numbers. Copernicus. 1996, Springer.

258. Jean, R.V., Phyllotaxis: a systemic study in plant morphogenesis. 2009: Cambridge University Press.

259. Khatib, 0., Real-time obstacle avoidance for manipulators and mobile robots. The international journal of robotics research, 1986. 5(1): p. 90-98.

260. Lumelsky, V.J. and A.A. Stepanov, Path-planning strategies for a point mobile automaton moving amidst unknown obstacles of arbitrary shape. Algorithmica, 1987. 2(1-4): p. 403-430.

261. Schelleng, J.C., The bowed string and the player. The Journal of the Acoustical Society of America, 1973. 53(1): p. 26-41.

262. Futagi, Y., Y. Toribe, and Y. Suzuki, The grasp reflex and moro reflex in infants: hierarchy of primitive reflex responses. International journal of pediatrics, 2012. 2012.

263. Stolarz, M., Circumnutation as a visible plant action and reaction: physiological, cellular and molecular basis for circumnutations. Plant signaling \& behavior, 2009. 4(5): p. 380-387.

264. Matuszek, C., et al. Learning to parse natural language commands to a robot control system. in Experimental Robotics. 2013. Springer.

265. Ferre, M., et al., Advances in telerobotics. Vol. 31. 2007, Madrid: Springer.

266. Martín-Barrio, A., et al., Modeling the soft robot Kyma based on Real-Time Finite Element Method. 2019. (under review)

267. Dutta, P., N. Rastogi, and K.K. Gotewal, Virtual reality applications in remote handling development for tokamaks in India. Fusion Engineering and Design, 2017. 118: p. 73-80.

268. Britton, N., et al. Lunar micro rover design for exploration through virtual reality tele-operations. in Field and Service Robotics. 2015. Springer.

269. Pessaux, P., et al., Towards cybernetic surgery: robotic and augmented reality-assisted liver segmentectomy. Langenbeck's archives of surgery, 2015. 400(3): p. 381-385.

270. Michalos, G., et al., Augmented reality (AR) applications for supporting human-robot interactive cooperation. Procedia CIRP, 2016. 41: p. 370-375.

271. Martínez, E., Diccionario Español de Ingeniería. 2014.

272. Roldán, J., et al., Multi-robot interfaces and operator situational awareness: Study of the impact of immersion and prediction. Sensors, 2017. 17(8): p. 1720 . 
273. Raskin, J., The humane interface: new directions for designing interactive systems. 2000, Boston: Addison-Wesley Professional.

274. Endsley, M.R. Design and evaluation for situation awareness enhancement. in Proceedings of the Human Factors Society annual meeting. 1988. SAGE Publications Sage CA: Los Angeles, CA.

275. Hart, S.G. and L.E. Staveland, Development of NASATLX (Task Load Index): Results of empirical and theoretical research., in Advances in Psychology. 1988, Elsevier. p. 139-183.

276. Sayers, C., Remote control robotics. 1999, New York, USA: Springer.
277. Daniel, W.W., Kolmogorov-Smirnov one-sample test. Applied Nonparametric Statistics, 1990. 2.

278. Endsley, M.R. Situation awareness global assessment technique (SAGAT). in Proceedings of the IEEE 1988 National Aerospace and Electronics Conference. 1988. IEEE.

279. Hart, S.G. NASA-task load index (NASA-TLX); 20 years later. in Proceedings of the human factors and ergonomics society annual meeting. 2006. Sage publications Sage CA: Los Angeles, CA.

280. Rüßmann, M., et al., Industry 4.0: The future of productivity and growth in manufacturing industries. Boston Consulting Group, 2015. 9(1): p. 54-89. 


\section{Declaration of originality}

I herewith declare that I have produced this dissertation without the prohibited assistance of third parties and without making use of aids other than those specified; notions taken over directly or indirectly from other sources have been identified as such.

The thesis work was conducted from September 2016 to May 2020 under the supervision of Prof. Antonio Barrientos Cruz at Universidad Politécnica de Madrid.

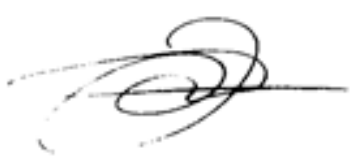

Andrés Martín Barrio 